Universidade de São Paulo

Instituto de Astronomia, Geofísica e Ciências Atmosféricas

Departament of Astronomy

Leonardo A. G. dos Santos

\title{
The rotational evolution of Sun-like stars and the influence of low-mass binary companions
}

A evolução rotacional de gêmeas solares e a influência de companheiras binárias de baixa massa

São Paulo 

Leonardo A. G. dos Santos

\section{The rotational evolution of Sun-like stars and the influence of low-mass binary companions}

A evolução rotacional de gêmeas solares e a influência de companheiras binárias de baixa massa

Dissertation presented to the Department of Astronomy of the Instituto de Astronomia, Geofísica e Ciências Atmosféricas at the Universidade de São Paulo as partial requirement to obtain the title of Master of Science.

Area: Astronomy

Advisor: Prof. Dr. Jorge Meléndez

Versão corrigida. O original encontra-se disponível na Unidade.

São Paulo 

I dedicate this work to my mother Benedita and to the memory of my father Eric. 



\section{Acknowledgements}

My most sincere thanks go to:

My family - Benedita, Julio and Marco - for the unconditional support they provided since I decided to become an astronomer, even though we passed through some challenging times since then;

Prof. Jorge Meléndez for the advising which went beyond scientific research;

My friends and colleagues Jhon Yana Galarza, Johnny Esteves, Lorenzo Spina, Marília Carlos and Raniere Menezes for the companionship and discussions;

The funding agency FAPESP for the confidence they deposited in me for the 2 and a half years of work, which was supported by their grants number 2014/26908-1 and 2016/01684-9;

Prof. Jacob L. Bean and Andreas Seifahrt, for providing a unique and life-changing opportunity;

Megan Bedell, Julian Stürmer, Benjamin Montet and Raphaëlle Haywood, for the important discussions, advice and being generally helpful with my research projects;

The institution and the staff at the Universidade de São Paulo and the University of Chicago for the hospitality and support;

The Python Software Foundation, for making the powerful Python programming language free and open;

Everyone involved in the building and operating of the HARPS spectrograph at the European Southern Observatory.

This dissertation was written in $\mathrm{AT}_{\mathrm{E}} \mathrm{X}$ using the class IAGTESE, for theses and dissertations from IAG. 

"Don't stay out of trouble."

Jehnny Beth 



\section{Resumo}

Dos Santos L. A., A evolução rotacional de gêmeas solares e a influência de companheiras binárias de baixa massa, Universidade de São Paulo, 2017, Dissertação de Mestrado.

O programa The Solar Twin Planet Search é um esforço sem precedentes na procura de planetas extra-solares em mais de 70 estrelas extremamente similares ao Sol. Ao longo desse programa, centenas de espectros ópticos de alta qualidade foram obtidos com o espectrógrafo HARPS, que está instalado no telescópio de 3,6 m do Observatório de La Silla. Além da busca de exoplanetas, estes dados são úteis para estudar as propriedades físicas de estrelas como o Sol. Estamos interessados em verificar se o Sol possui uma rotação regular para sua idade quando comparado com estrelas estritamente similares a ele, como que a rotação de gêmeas solares evolui com o tempo e se a rotação dessas estrelas é influenciada pela presença de companheiras estelares. Conclusões anteriores na regularidade da rotação solar são conflitantes, e esta é a primeira vez que uma amostra grande de gêmeas solares com dados espectroscópicos de alta qualidade é usada para esclarecer essa questão. Nossos resultados sugerem que o Sol de fato rota regularmente para sua idade, o que favorece o uso da rotação solar para calibrar a girocronologia - a estimativa de idades estelares a partir de sua rotação. No entanto, tais resultados também implicam em um processo de evolução rotacional que satura depois da idade solar, constituindo um desvio da amplamente usada relação de Skumanich e apresentando um desafio para a girocronologia. Nós identificamos 18 sistemas binários na amostra de gêmeas solares, das quais apenas três mostram rotações elevadas para suas idades. Os parâmetros orbitais das binárias foram estimados a partir da variação de suas velocidades radiais, e os resultados mostram que suas companheiras espectroscópicas possuem períodos orbitais variando de alguns poucos até muitos anos. Concluimos que a presença de companheiras do tipo anãs vermelhas ou marrons 
em períodos orbitais moderados não influenciam a evolução rotacional desses sistemas. As peculiaridades de HIP 19911, HIP 67620 e HIP 103983 podem ser completamente explicadas por contaminação espectral de suas companheiras.

Palavras-chave: Sol. Estrelas. Rotação estelar. Estrelas binárias. Gêmeas solares. 


\section{Abstract}

Dos Santos L. A., The rotational evolution of Sun-like stars and the influence of low-mass binary companions, Universidade de São Paulo, 2017, Master Dissertation.

The Solar Twin Planet Search program is an unprecedented effort that aimed to procure extrasolar planets in more than 70 stars extremely similar to the Sun. In the course of this program, hundreds of high-quality optical spectra were obtained for these stars using the HARPS spectrograph, which is fed by the ESO La Silla $3.6 \mathrm{~m}$ telescope. Beyond the search for exoplanets, the data are invaluable to study the physical properties of Sun-like stars. Particularly in this dissertation, we are interested in verifying if the Sun possesses a regular rotation for its age among stars that are strictly similar to it, how the rotation of solar twins evolve with age and if the rotation of Sun-like stars is influenced by the presence of stellar mass companions. Previous conclusions on the regularity of the Sun's rotation have been conflicting, and this is the first time such a large sample of solar twins with high quality spectroscopic data is used to clarify this puzzle. Our results suggest that the Sun is indeed a regular rotator for its age, which favors the use of the solar rotation to calibrate gyrochronology - the estimation of stellar ages from their rotation. However, these results also imply a rotational evolution process that saturates after the solar age, constituting a departure from the widely used Skumanich relation and posing a challenge for gyrochronology. We securely identified 18 binary or multiple systems in the solar twin sample, of which only three display enhanced rotation for their ages. I estimated the orbital parameters of the binaries from their radial velocity variations, and the results show that their spectroscopic companions lie at orbital periods varying from a few to several years. I conclude that the presence of red or brown dwarf companions at moderate to long orbital periods do not influence the evolution of rotation in these systems, and therefore the main stars should evolve as single in this 
regard. The peculiarities in HIP 19911, HIP 67620 and HIP 103983 can be fully explained by spectral contamination from their companions.

Keywords: Sun. Stars. Stellar rotation. Binary stars. Solar twins. 


\section{List of Figures}

1.1 Sun spots and the rotation of the Sun . . . . . . . . . . . . 22

1.2 HR-diagram of the solar twins in this study f . . . . . . . . . . . 24

1.3 Mass-orbital period diagram of the exoplanets discovered thus far . . . . . . 25

1.4 Granulation observed in the solar surface . . . . . . . . . . . . . . . . . . 32

1.5 The rotational profile of a star $\ldots \ldots \ldots \ldots \ldots$

2.1 Variation of the MIKE spectral resolving power . . . . . . . . . . . . 37

2.2 Isochronal age determination for the stars in the Solar Twin Planet Search . . . . 43

3.1 Spectral line brodening in stars with different $v \sin i \ldots \ldots \ldots 1$

3.2 Spectral line profile fitting $\ldots \ldots \ldots \ldots \ldots \ldots$

3.3 Linear trend between macroturbulence velocity and surface gravity . . . . . . . . 55

3.4 Comparison between CCF and synthetic spectra methods for measuring $v \sin i$. . 58

3.5 Projected rotational velocity of solar twins as a function of their age . . . . . . 59

3.6 The rotational evolution of the stars in the program $\ldots \ldots \ldots$. . . . . . 60

4.1 Orbital solutions of newly discovered solar twin binaries . . . . . . . . . 73

4.2 Orbital solutions of known solar twin binaries . . . . . . . . . . . . . . . 74

4.3 Double lines in the spectra of HIP 19911 and HIP $103983 \ldots \ldots$. . . . . . . . 76

4.4 Radial velocities and solutions for RV curvature-only binaries . . . . . . . . . 80

4.5 Radial velocities of HIP $14501 \ldots \ldots$. . . . . . . . . . . . . 80

4.6 Posterior probability distribution of the companion mass for HIP $64150 \ldots$. . . . 81

4.7 Mass ratios and orbital periods of binary stars in the solar neighborhood . . . . . 84

5.1 Models of the radii and rotational periods of solar twins . . . . . . . . . . . . 90 



\section{List of Tables}

2.1 Technical specifications of the MIKE spectrograph $\ldots \ldots$. . . . . . . . 36

2.2 Technical specifications of the HARPS spectrograph $\ldots \ldots \ldots$

3.1 Line list used in the projected stellar rotation measurements . . . . . . . . . . 52

3.2 Macroturbulence velocities of a subsample of solar twins . . . . . . . . . . 55

4.1 Additional radial velocities from other programs and instruments . . . . . . . 68

4.2 Orbital parameters of the short-period binaries . . . . . . . . . . . 73

4.3 Orbital parameters of the RV curvature-only binaries $\ldots \ldots \ldots$

4.4 RV slopes and masses for the linear trend binaries . . . . . . . . . . . 83

A.1 Ages, $v \sin i$ and stellar parameters of the solar twins and the Sun . . . . . . 114 



\section{Contents}

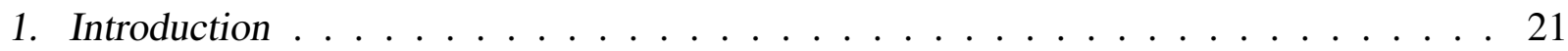

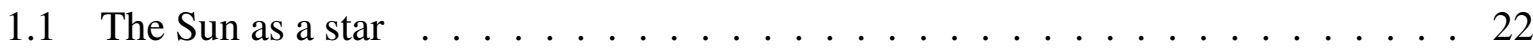

1.1.1 Solar twins and solar analogues . . . . . . . . . . . . 23

1.1.2 Is the Sun a Sun-like star? . . . . . . . . . . . . . . . . . 25

1.2 Rotational evolution explained by stellar winds . . . . . . . . . . 26

1.3 Gyrochronology and the relation between rotation and stellar activity . . . . . . 28

1.4 Observation of stellar rotation using high-resolution spectra . . . . . . . . . 30

1.4.1 Thermal broadening . . . . . . . . . . . . . . . 31

1.4.2 Micro- and macroturbulence . . . . . . . . . . . . . 31

1.4.3 Instrumental profile . . . . . . . . . . . . . . 32

1.4.4 Stellar rotation $\ldots \ldots \ldots \ldots \ldots$

2. The data and observational constraints . . . . . . . . . . . . . . 35

2.1 Data from the MIKE spectrograph . . . . . . . . . . . . . 35

2.2 Data from the HARPS spectrograph . . . . . . . . . . . . . . . . 37

2.3 Other programs . . . . . . . . . . . . . . . . . . 39

2.3.1 Data from the literature . . . . . . . . . . . . . . . 39

2.3.2 Data from the ELODIE and SOPHIE spectrographs . . . . . . . . . . 40

2.3.3 Data from the HIRES spectrograph . . . . . . . . . . . . . . . . 40

2.3.4 Data from the Lick Planet Search . . . . . . . . . . . . . . . . . 40

2.3.5 Data from the Anglo-Australian Telescope Planet Search . . . . . . . . . 40

2.4 The isochronal ages and masses of the solar twin sample . . . . . . . . . 41

2.4.1 Computing probability distributions . . . . . . . . . . . . . 41 
2.4.2 Concerns in the use of isochronal ages . . . . . . . . . . . . . 42

3. Rotational evolution of Sun-like stars . . . . . . . . . . . . . . . . . . . 47

3.1 Abstract of this chapter . . . . . . . . . . . . . . . 47

3.2 The role of the Sun in our understanding of rotational evolution . . . . . . . . . 48

3.3 Working sample . . . . . . . . . . . . . . . 50

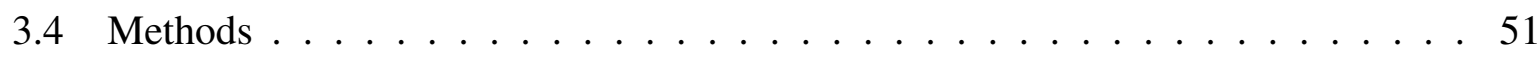

3.4.1 Macroturbulence velocities . . . . . . . . . . . . 52

3.4.2 Rotational velocities . . . . . . . . . . . . . 56

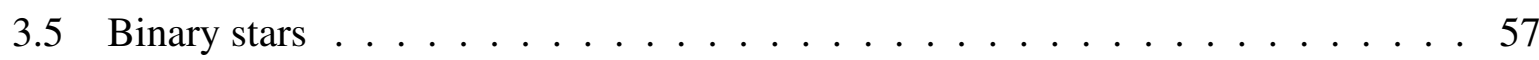

3.6 The rotational braking law . . . . . . . . . . . . . . 59

3.7 Conclusions of this chapter . . . . . . . . . . . . . 62

4. New and improved orbital constraints of solar twin binaries . . . . . . . . . . . 65

4.1 Abstract of this chapter . . . . . . . . . . . . . . . 65

4.2 Do binary solar twins follow a distinct evolutionary path? . . . . . . . . . 66

4.3 Radial velocities . . . . . . . . . . . . . . . . 68

4.4 Methods . . . . . . . . . . . . . . . . . . . 69

4.4.1 Binaries with well-sampled orbits . . . . . . . . . . . . . . . . 69

4.4.2 Binaries with partial orbits . . . . . . . . . . . . 70

4.5 Results . . . . . . . . . . . . . . . . . . 72

4.5.1 Withdrawn binary candidates . . . . . . . . . . . 72

4.5.2 Solar twins with new companions . . . . . . . . . . . . 72

4.5.3 The peculiar binaries . . . . . . . . . . . . 75

4.5.3.1 HIP $19911 \ldots \ldots \ldots \ldots \ldots$

4.5.3.2 HIP $67620 \ldots \ldots \ldots \ldots \ldots 77$

$4.5 .3 .3 \quad$ HIP $103983 \ldots \ldots \ldots \ldots$

4.5.4 Other binaries with updated orbital parameters . . . . . . . . . 79

4.5.5 Considerations on multiplicity statistics $\ldots \ldots \ldots$. . . . . . . 83

4.6 Conclusions of this chapter . . . . . . . . . . . . . 85

5. Concluding marks . . . . . . . . . . . . . . . . . 87

5.1 Summary of this dissertation $\ldots \ldots \ldots \ldots$. . . . . . . . . . 87 
5.2 Future outlook . . . . . . . . . . . . . . . . . 89

5.2.1 Rotation of Sun-like stars . . . . . . . . . . . . . . . . . . . . 89

5.2.2 Models of stellar atmospheres and evolution . . . . . . . . . . . . 89

5.2.3 Stellar activity and the search for habitable planets . . . . . . . . . 90

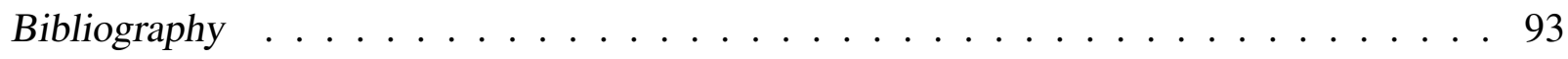

Appendix

A. The stellar parameters of the solar twins . . . . . . . . . . . 113

B. First- and second-author articles . . . . . . . . . . . . . . . . 119 



\section{Chapter 1}

\section{Introduction}

In a nutshell, stars are objects constituted of hot gas, mostly hydrogen and helium and containing traces of other elements. They are born from clouds of gas and dust that collapse due to gravity. This causes a gradual increase in the temperature and pressure of the inner regions, which eventually develop the conditions necessary to start nuclear fusion. These reactions produce an outwards pressure against gravity and, when these forces balance each other out, we have a stable main sequence star, the longest phase that all stars go through in their lifetimes.

One of the most fundamental aspects of the physics of a Sun-like star how it evolves with time, particularly in its rotation. The quantity of rotational movement affects countless processes in the inner regions and the surface of a star (e.g., see Fig. 1.1). The rotation of a star is related to the initial angular momentum of the cloud from which it was born. Due to many phenomena, however, some of them still nebulous to astrophysics, the stellar rotation changes with time, even during the main sequence phase. Additionally, it is possible that stars that are very similar to each other have different rotational rates. We, therefore, ask the question: is the Sun common in its rotation when compared to similar stars?

My dissertation aims to shine a light on this question. We compare the rotational velocities of solar twins with what we observe in the Sun using the largest sample of such stars ever compiled for this kind of study. In total, 81 solar twins were observed in extremely high resolution with the HARPS spectrograph, which is fed by the $3.6 \mathrm{~m}$ telescope at La Silla Observatory, operated by the European Southern Observatory (ESO). One of our main objectives is to find a consistent relation between similar stars. As many of the measurements and inferences about the universe are based on our knowledge of the Sun - such as the the chemical evolution of our Galaxy and the physical properties of exoplanets -, it is crucial that we understand it well enough to have a solid foundation for astrophysics. 


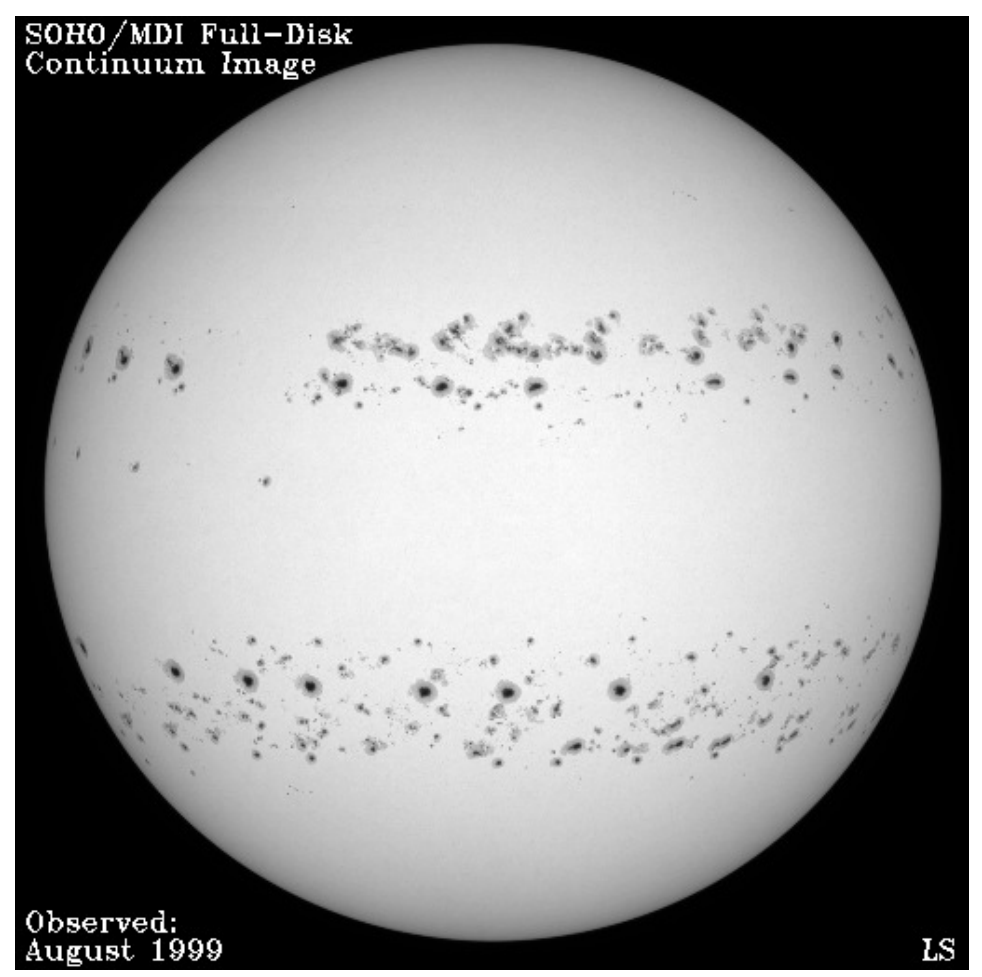

Figure 1.1: One way to study stellar rotation is through the monitoring of star spots. This image obtained by the instrument MDI at the Solar and Heliospheric Observatory (SOHO) shows the tracks of spots on the surface of the Sun in August 1999. The synodic period of Carrington rotation of the Sun is approximately 27 days. Credit: Louis Strous (LMSAL), SOHO-MDI Consortium, ESA, NASA.

\subsection{The Sun as a star}

The Sun is the only star we can study to a high level of detail due to its proximity to Earth, which is of course a blessing in the quest towards understanding how it works, but a curse in disguise when we need to put the Sun in context with other stars. This is an essential step to achieve the objectives of my study, and it is far from trivial.

The ways we observe the Sun and other stars are fundamentally different. The first is bright and has an extended disk in the sky, so solar telescopes are generally applied. The second are point-sources with a wide range of brightness, which requires us to observe them with a range of different telescopes. When we use different instruments, systematics start to matter.

The most straightforward solution to this problem is to observe the Sun through the reflected light of other bodies in the Solar System, as long as we perform corrections for the properties intrinsic for these bodies (such as rotation and motion in relation to the Sun and to Earth). This allows us to observe our star with the same instruments as the others. 
With the systematics problem solved, we are limited now by how well we know the other stars. In regards to direct measurements, such as colors, apparent magnitude, proper motion and parallax (i.e., distance), we are mainly dealing with stochastic errors intrinsic to the precision allowed by our instruments - assuming they are perfectly calibrated. Other stellar parameters, such as mass, effective temperature and age are model dependent, i.e., they depend not only on the accuracy of our measurements, but also on the assumptions and approximations we adopt in our models.

One way to circumvent the limitations of our models is to narrow down comparisons of the Sun to stars strictly similar to it. Because of this similarity, uncertainties in the theoretical treatment or in physical constants do not play a significant role in the information we are able to extract from the comparison. This is where solar twins and solar analogues come into play.

\subsubsection{Solar twins and solar analogues}

We know the stellar parameters of the Sun to a high precision level, thus if we limit ourselves to stars that have the same parameters within the uncertainties of the solar values, we will not have a sizeable sample to perform any kind of comparison, if we are able to find a sample at all ${ }^{1}$. In a sense, the stellar parameters are like fingerprints: there are no stars with the same sets of values. We need, thus, to throw a wider net to be able to capture other stars that are still similar, but different enough to constitute a large sample.

There are various definitions for Sun-like stars, such as solar analogues and solar twins, and they differ in the level of similarity with our star. Twins are informally described as those that have effective temperature $\left(T_{\text {eff }}\right)$, surface gravity $(\log g)$ and metallicity $([\mathrm{Fe} / \mathrm{H}])$ inside the intervals $\pm 100 \mathrm{~K}, \pm 0.1 \mathrm{dex}[\mathrm{cgs}]$ and $\pm 0.1 \mathrm{dex}$, respectively, around the solar values (Ramírez et al., 2014). Analogues, on the other hand, have wider intervals around solar values for the stellar parameters. As seen in Fig. 1.2, I am also including stars in the sample that are not strictly solar twins, but they are still inside one or more parameter intervals in the definition I use; this is necessary to increase the number of stars in the sample and broaden the coverage in stellar age.

In order to leverage the similarity of the solar twins to the Sun to obtain more precise and accurate estimates of their stellar parameters, there have been several studies in the recent past that applied differential analysis. See Chapter 16 of Gray (2005) for a detailed description of

\footnotetext{
${ }^{1}$ Strictly speaking, there may be stars exactly identical to the Sun, but the chances are very low.
} 


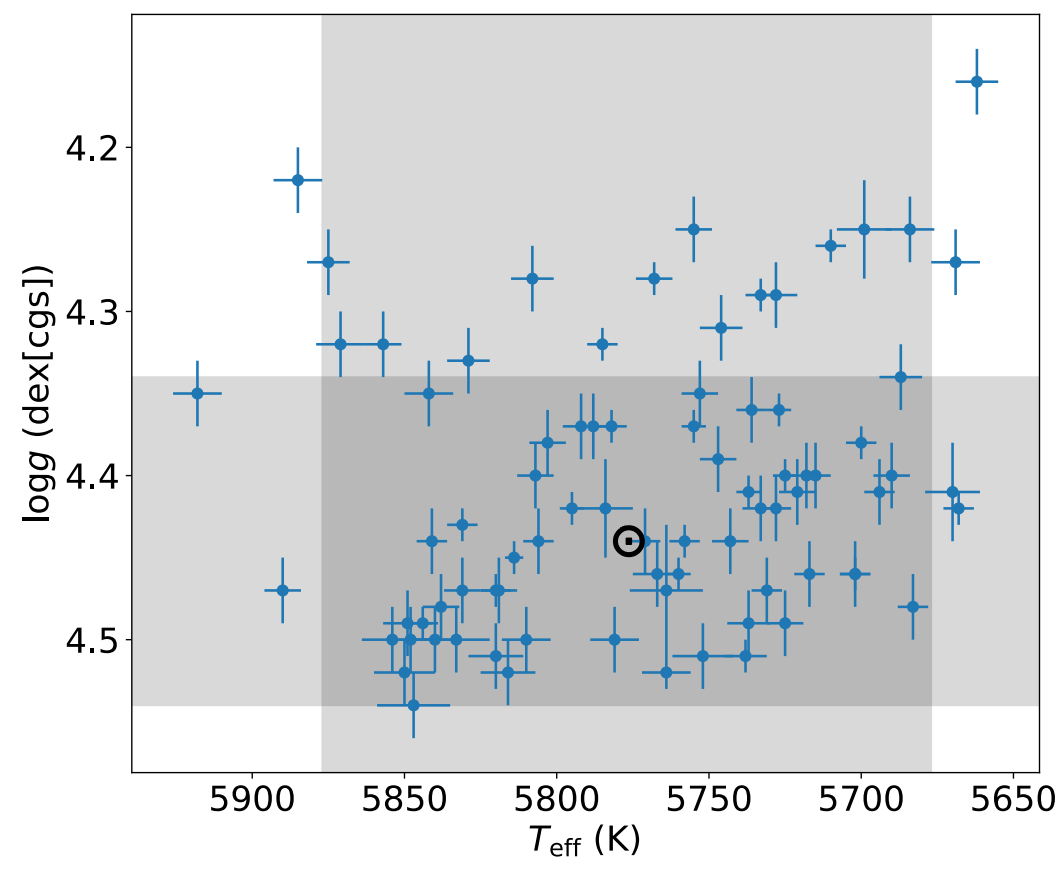

Figure 1.2: Hertzprung-Russel diagram of the solar twins sample I analyzed in the present study. The Sun is represented by the usual $\odot$ symbol. The gray regions correspond to the definition of solar twins I used.

the methodology. It has been shown that this procedure guarantees $\sim 0.01$ dex precision in the relative chemical abundances derived using standard model atmosphere methods and that the systematic uncertainties of that analysis are negligible within the solar twin stellar parameter ranges (Bedell et al., 2014; Biazzo et al., 2015; Saffe et al., 2015; Yana Galarza et al., 2016).

The origin of such high precision for the stellar parameters (as seen in the HR diagram in Fig. 1.2) lies in the precise determination of the differential abundances of iron in the atmospheres of the solar twins. In the end, the uncertainties of the stellar parameters will depend on (roughly in order of importance):

- Errors in the measurement of the equivalent widths of several spectral lines of iron

- Errors emerging from the imperfectness of stellar atmosphere models - which would dominate if we were working with an absolute analysis

- Noise in the observed spectra

- The function used to fit the spectral lines (generally a Gaussian or a Lorentzian) 


\subsubsection{Is the Sun a Sun-like star?}

This somewhat metaphysical question has been one of the centers of attention of stellar astrophysics since the seminal discovery of the first extrasolar planet (or exoplanet) around a main sequence star (Mayor and Queloz, 1995). To this date, thousands of exoplanets and exoplanet candidates have been discovered thanks to the Kepler mission (see, e.g., Borucki et al., 2009; Kane et al., 2016; Morton et al., 2016) and several other programs (see Fig. 1.3).

From these results emerged a conclusion that the planetary systems we observe in the solar neighborhood are, for the most part, completely different from the Solar System (e.g., Batalha, 2014; Rogers, 2015; Steffen and Coughlin, 2016). What could possibly explain such disparity? Is the Sun a unique star?

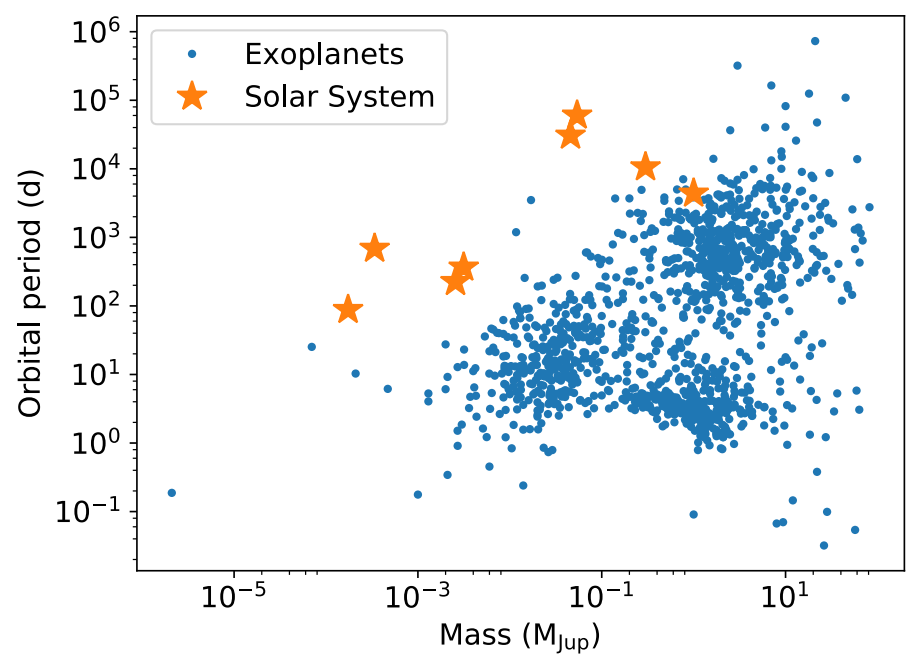

Figure 1.3: Mass-orbital period diagram of all the confirmed 2702 exoplanets as of April 24th 2017. The planets of the Solar System (orange stars) are clearly very different from the distribution of exoplanets. Source of data: The exoplanet. eu portal.

In this regard, Gustafsson (2008) provides an overview into the issue, pointing out that the Sun is indeed a regular star in various aspects, but abnormal in its mass, variability amplitude and multiplicity. On the other hand, Soderblom (2010) argues that, because we know the Sun to such a high degree, the null hypothesis should be that the Sun is unusual for its mass, chemical composition and age. Current efforts in answering this question have focused on precisely characterizing the chemical composition and physical properties of the Sun and Sun-like stars (e.g., Meléndez et al., 2009; do Nascimento et al., 2014; Leão et al., 2015; Haywood et al., 2016; Egeland et al., 2017). 
In the past, it has been suggested that there could be a correlation between the slow rotation of a star and the formation of massive Jupiter-like planets (e.g., Smith, 1979), which could act as an angular momentum sink. However, if that was the case, the Sun should be a typical rotator, since we have seen in the latest years that the presence of planets in other cool stars is ubiquitous. Ceillier et al. (2016) showed that small planets do not affect the rotational evolution of dwarf stars such as the Sun, while only more massive close-in planets can influence the rotation of a Sun-like star (Bolmont et al., 2012; Ferraz-Mello et al., 2015).

Thus, it is natural to think that research about the rotation and magnetic activity of Sun-like stars provides important clues in the puzzle of planetary system evolution. We are only able to understand a planet as much as we understand its star, so our next step is to take a look at how Sun-like stars evolve in their rotation.

\subsection{Rotational evolution explained by stellar winds}

During the pre-main sequence phase of the evolution of a star, simple angular momentum conservation predicts that the rotation of a protostar will increase as the following equation:

$$
v_{\mathrm{eq}}=\frac{R J}{I},
$$

where $J$ is the angular momentum, $I$ is the moment of inertia, $v_{\mathrm{eq}}$ is the rotational velocity in the equator of the star and $R$ is the stellar radius.

When a star begins the main sequence phase, it enters a stable phase during which the stellar radius evolve slowly in the timescale of the nuclear burning. The first observational evidence for rotational evolution of pre-main sequence and main sequence Sun-like stars comes from studies such as Kraft (1967), Skumanich (1972) and Vogel and Kuhi (1981).

The theoretical foundation of rotational evolution lies in the conservation of mass and angular momentum of a rotating star. Considering a rigidly rotating and constant density spherical shell, the conservation of mass and angular momentum requires that the shell spins down as it expands. According to Charbonneau (1992), if the shell expands with a forced constant angular velocity, then whatever enforces this state must supply a relative excess of angular momentum

$$
\frac{\Delta J}{J_{0}}=\frac{J_{1}-J_{0}}{J_{0}}=\left(\frac{R_{1}}{R_{0}}\right)^{2}-1,
$$


where $J$ is the angular momentum, $R$ is the shell radius, and the indices 0 and 1 correspond to the initial and final state, respectively.

For stars like the Sun, the movement of large convective cells near their surface produce magnetic fields; the stellar rotation, in turn, makes field lines wrap around the star, creating a stellar dynamo (Charbonneau, 2014). The magnetic fields anchor on the stellar surface and force co-rotation to large distances, thus providing excess angular momentum (Schatzman, 1962; Mestel, 1984). Outside the stellar surface, the wind will conserve the angular velocity up to a critical distance - the co-rotation radius - where it decouples from the magnetic field and carries away the angular momentum provided by the magnetic field in the first place.

Because the winds are thermally driven (Parker, 1958), the wind velocity increases with distance; the Alfvén velocity, on the other hand, decreases with distance because it is proportional to the magnetic field strength. The angular momentum loss is thus proportional to the strength of the magnetic field of the star (Charbonneau, 1992). We define the co-rotation or Alfvén radius as the distance where the Alfvén velocity is equal to the wind velocity.

For radial magnetic fields, the braking of a star can be decribed by

$$
\frac{d J}{d t}=\frac{2}{3} \frac{d M}{d t} R^{2} \Omega\left(\frac{r_{\mathrm{A}}}{R}\right)^{2}
$$

where $\Omega$ is the rotational rate and $r_{\mathrm{A}}$ is the Alfvén radius (Mestel, 1984). Different geometries of the magnetic field can be scaled in relation to the radial geometry, yielding

$$
\frac{d J}{d t}=\frac{2}{3} \frac{d M}{d t} R^{2} \Omega\left(\frac{r_{\mathrm{A}}}{R}\right)^{n}
$$

where $n=2$ for radial geometry and $n=3 / 7$ for dipole geometry (Kawaler, 1988). For a purely radial field, we have that

$$
\frac{r_{\mathrm{A}}}{R}=B_{0} R\left(\dot{M} v_{\mathrm{A}}\right)^{-1 / 2},
$$

where $B_{0}$ is the magnetic field strength. Assuming that $B_{0}$ depends solely on the radius and rotation rate of the star, Kawaler (1988) proposed the following expression for the angular momentum loss in terms of the stellar parameters and four free parameters

$$
\frac{d J}{d t}=-K_{\mathrm{W}} \Omega^{1+(4 a n / 3)}\left(\frac{R}{R_{\odot}}\right)^{2-n}\left(\frac{\dot{M}}{-10^{-14} M_{\odot} y r^{-1}}\right)^{1-(2 n / 3)}\left(\frac{M}{M_{\odot}}\right)^{-n / 3}
$$


where $K_{\mathrm{W}}=2.035 \times 10^{33}\left(24.93 K_{\mathrm{V}}^{-1 / 2}\right)^{n} K_{\mathrm{B}}^{4 n / 3}$ and $K_{\mathrm{V}}, K_{\mathrm{B}}, n$ and $a$ are the free parameters that represent our ignorance of the stellar magnetic fields. This approach has been widely used to model the rotational evolution of Sun-like stars and, as we see in the discussion of Chapter 3, is successful in reproducing the general rotational velocities trends observed for these stars.

Assuming that $I$ and $R$ are approximately constant during the main sequence phase, Eqs. 1.1 and 1.6 can be combined to yield the following relation:

$$
v_{\mathrm{eq}} \propto t^{-\frac{3}{4 a n}}
$$

which encapsulates the basic physics of rotational evolution of Sun-like stars in the main sequence. In practice, the values of the parameter $n$ and $a$ dictate the level of dependence of $d J / d t$ on $B_{0}$ and the rotation rate, respectively, if all other parameters are kept constant. This means that, for example, larger values of $n$ will produce earlier rotational braking effects.

The models by Kawaler (1988) predict that the rotational velocities after $\sim 10^{8} \mathrm{yr}$ are essentially independent of the initial angular momentum of the pre-main sequence star (see Fig. 4 on their paper). Furthermore, observational evidence shows that the rotation of main sequence stars converge towards a similar value independent of their initial angular momenta (see, e.g., Barnes, 2010; Gallet and Bouvier, 2013). In other words, the surface rotation $P$ of a cool main sequence star depends only in its mass $M$ and age $t$, i.e., $P=P(M, t)$ (see Meibom et al., 2011, and references therein). From this conclusion emerged the field of gyrochronology, which sought to empirically measure the age of main-sequence stars based on their masses and rotational periods (see Section 1.3).

\subsection{Gyrochronology and the relation between rotation and stellar activity}

The evolution of stellar rotation during the pre-main sequence and main sequence phases has been the target of incisive research for decades. In a pioneering study on the subject, Skumanich (1972) used only three data points from the Pleiades and Hyades open clusters and the Sun to infer that, for main sequence (MS) stars, the $\mathrm{Ca}^{+}$emission luminosity in a $1.1 \AA$ band - a proxy for stellar activity - decays in a rate proportional to the square root of the stellar age (Eq. 1.8). Additionally, since the emission intensity is proportional to the mean magnetic field at the surface of the star, which is in turn proportional to the rotational velocity, the author concludes that the rotation of MS stars also decays with the same proportionality to the stellar age. This relation is 
now known as the Skumanich law (compare it with Eq. 1.7):

$$
v \propto t^{-1 / 2}
$$

The first strong empirical evidence for the correlation between stellar activity and rotation emerged from the results obtained by Wilson (1978). In this seminal study, the author observed that main sequence stars ranging from spectral types F5 to M2 exhibited activity cycles that were similar to the 11-year cycle of the Sun. According to Noyes et al. (1984), this correlation is an expected outcome because the cyclical amplification of the magnetic fields emerges from: i) the helicity produced by the action of rotation on convection, and ii) the shear produced by differential rotation. Skumanich (1972) also observed that the abundances of Li seem to follow the $t^{-1 / 2}$ law, but only for ages up to the Hyades open cluster age (at that time being estimated at $400 \mathrm{Myr}$ ), at which point the $\mathrm{Li}$ abundance decays exponentially.

The inference of a star's age from its magnetic activity or rotation is now known as gyrochronology. Meibom et al. (2015) point out that there are measurements of rotational periods from starspots for field stars, but their ages are not precise enough for use in gyrochronology, and thus the calibration must necessarily be done in stellar clusters.

It is also necessary to compare ages obtained by gyrochronology with those obtained by other methods in order to validate the first. Other methods include the isochronal ages (e.g., Lachaume et al., 1999), asteroseismic ages (see Lebreton and Montalbán, 2009; Silva Aguirre et al., 2015) and $\mathrm{Li}$ abundances (see Carlos et al., 2016, and references therein). Some peculiar systems, such as those that host a white dwarf (WD) can also have their age precisely and accurately measured using the WD cooling age method (Bergeron et al., 2011).

In a recent study with stars very similar to the Sun (see Section 1.1.1), Freitas (2017) analyzed the relation between the observed chromospheric activity index $R_{\mathrm{HK}}^{\prime}$ and isochronal stellar ages obtained from a high-precision differential analysis (see Chapter 2), and mainly concluded that: i) the Sun has a regular activity for its age, and ii) chromospheric ages for Sun-like stars renders reliable ages only when they are below the solar age; for older stars the chromospheric activity index plateaus, thus precluding a precise age determination. Similar results have been obtained by van Saders et al. (2016), who used seismic ages and rotational periods from Kepler to calibrate the rotation-age relation. On the other hand, Lorenzo-Oliveira et al. (2016) were able to obtain a consistent chromospheric age for the old (7.1 Gyr) open cluster NGC 188, although stressing that further work is needed. 
As we saw in Section 1.2, the Skumanich law, or at least a Skumanich-like relation, emerges naturally from the conservation of mass and angular momentum and a minimal number of assumptions (see Eq. 1.7). Departures from this relation can be traced to differing configurations or regimes of the stellar magnetic field, and are usually described in terms of the Rossby number:

$$
R o=\frac{P}{\tau_{\mathrm{cz}}},
$$

where $P$ is the rotational period and $\tau_{\mathrm{cz}}$ is the characteristic time of the convective zone overturn and also a proxy for the depth of the convective zone. As explained in Section 1.2, the convection zone in a star dictates the strength and configuration of the magnetic fields, and they vary for differing stellar masses. Several studies in the past have used the Rossby number to formulate the rotational and activity evolution of low-mass stars, of which Noyes et al. (1984) and Barnes (2010) are recommended reading.

\subsection{Observation of stellar rotation using high-resolution spectra}

Because of the distances involved, it is rare to have a star for which we can resolve its disk. Nevertheless, it is still possible to detect and measure the rotation of a star without the need of a high-spatial resolution telescope. There are various methods that are generally used to infer about the rotation of a star, of which the most used ones are:

1. Brightness variability: The movement of stellar spots in the surface of a star as it rotates causes its brightness to vary periodically, from which we can extract the rotational period. This method has been extensively applied accross many types of stars in the literature (e.g., McQuillan et al., 2013).

2. Variability of the $\mathrm{Ca}$ II $\mathrm{H} \& \mathrm{~K}$ emission lines: they are directly related to the activity of the star, which is in turn coupled with the surface rotation (see Section 1.3). The variation of the strength of these lines is thus a proxy for stellar rotation.

3. Spectral line broadening: It consists on modelling the behavior of spectral line widths with varying rotation speeds and comparing with the observed lines. This method is particularly challenging for Sun-like stars because they rotate slowly enough for the signal to be lost in noisy data, and there are competing factors that contribute to line broadening. In my study, however, I was able to successfully apply this method because of the high quality of the 
obtained spectra, which allowed me to disentangle rotation from other phenomena. This dissertation carefully describes the methodology and discusses the results of the study.

Spectral lines were independently discovered by the German physicist Joseph von Fraunhofer in 1814, and they result from the interaction of light emerging from the stellar interior with the star's superficial layers. The dominant line broadening processes for my work are outlined below. A theoretical description of this phenomenon that is sufficiently complete to understand my study can be found on Chapters 11, 12 and 13 in Gray (2005).

\subsubsection{Thermal broadening}

Atoms in the gaseous phase have random velocities that originate from their thermal motion. These velocities follow a Gaussian or Maxwellian distribution, and contribute to spectral line broadening due to Doppler shift arising from their movement along the line of sight. The energy removed from a unit intensity beam in wavelength space follows the relation

$$
\alpha d \lambda \propto \frac{\lambda_{0}^{2}}{c} \frac{1}{\Delta \lambda_{\mathrm{D}}} \exp \left[-\left(\frac{\lambda-\lambda_{0}}{\Delta \lambda_{\mathrm{D}}}\right)^{2}\right] d \lambda,
$$

where $\alpha$ is defined as the thermal absorption coefficient, $\lambda_{0}$ is the central wavelength of the spectral line and $c$ is the speed of light. $\Delta \lambda_{\mathrm{D}}$ is given by

$$
\Delta \lambda_{\mathrm{D}}=\frac{\lambda_{0}}{c}\left(\frac{2 k T}{m}\right)^{1 / 2}
$$

where $k$ is Boltzmann's constant, $m$ is the atomic mass and $T$ is the temperature of the gas.

\subsubsection{Micro- and macroturbulence}

Velocity fields in stellar photospheres contribute to spectral line broadening mainly by the movement of convective cells and rotation. The first emerges in the surface of a star in the form of granulation, and it is easily visible in the Sun (see Fig. 1.4). These movements are radial, thus they have no contribution in the stellar limbs, and are strongest in the stellar center.

When the convective cells are small (compared to the photon mean free path), the line of sight penetrates through many cells, which in turn will have a velocity distribution similar to moving particles (i.e., approximately a Gaussian distribution):

$$
N(v) d v=\frac{1}{\pi^{1 / 2} \xi} e^{-(v / \xi)^{2}} d v
$$




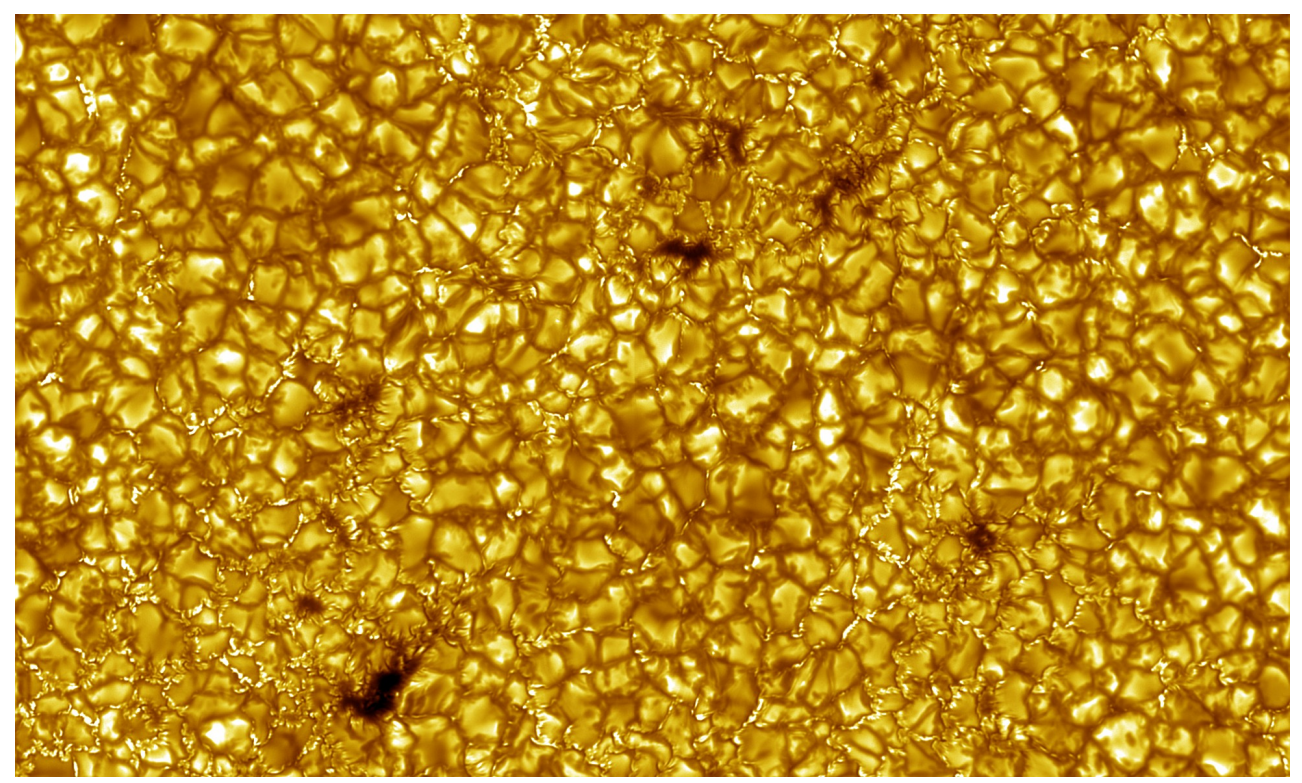

Figure 1.4: High-resolution Ca II $396.37 \mathrm{~nm}$ wideband image of the Sun observed with the Swedish 1-m Solar Telescope (SST) at La Palma. It clearly shows the solar granulation, a dense structure observed in the surface of our star. They result from the velocity fields in the solar photosphere, and are unrelated to the dark spots. Credit: Vasco Henriques, Institute for Solar Physics of the Swedish Research Counsil, Stockholm University, Instituto de Astrofísica de Canarias.

where $v$ is the convective cell velocity and $\xi$ is called the microturbulence velocity.

If the convective cells are large, they must be trated as individual sources of spectra. The usual way of computing their contribution to line broadening is to use the radial-tangential anisotropic macroturbulence approximation (Gray, 1975). This model consists on decomposing the distribution of convective cell velocities on a radial component and a tangential component:

$$
\Theta(\Delta \lambda)=\frac{A_{\mathrm{R}}}{\pi^{1 / 2} \zeta_{\mathrm{R}} \cos \theta} e^{-\left(\Delta \lambda / \zeta_{\mathrm{R}} \cos \theta\right)^{2}}+\frac{A_{\mathrm{T}}}{\pi^{1 / 2} \zeta_{\mathrm{T}} \sin \theta} e^{-\left(\Delta \lambda / \zeta_{\mathrm{T}} \sin \theta\right)^{2}}
$$

where $A_{\mathrm{R}}$ and $A_{\mathrm{T}}$ are the stellar surface areas corresponding to radial and tangential motion, respectivelly, $\zeta$ are the macroturbulence velocities, $\theta$ is the angle between line of sight and the direction of motion, and $\Delta \lambda$ is the wavelength shift from the line center.

\subsubsection{Instrumental profile}

The intrinsic spectra of astronomical objects, when observed through a spectrograph, are convolved with the profile of our instruments, and this profile accounts mainly for effects due to the entrance-slit width, the detector pixel size and optical aberrations. Generally, the higher the resolving power (also known as spectral resolution) of a spectrograph, the finer the instrumental 
profile is, allowing us to study the spectra in more detail. On the other hand, the higher resolving power causes a larger wavelength dispersion, which in turn is problematic for fainter objects. Thus, astronomers often need to weight in the trade-offs between higher resolutions and noise.

My study involves the analysis of spectra of very bright stars $(6<V<9)$, which allowed us to use the highest resolving power available on the spectrograph HARPS. Instrumental profiles are often complicated to model and to infer from real data. Generally, an acceptable approximation is a Gaussian function, which is what I adopted in this work.

\subsubsection{Stellar rotation}

The contribution of line broadening by stellar rotation is outlined below, following the formalism proposed by Gray (2005). If we assume a spherical star rotating as a rigid body, we have that the normalized line profile can be written as

$$
\frac{F_{v}}{F_{\mathrm{c}}}=H(v) * G(v)=H(\Delta \lambda) * G(\Delta \lambda),
$$

where $F_{v}$ is the flux in the spectral line, $F_{\mathrm{c}}$ is the flux in the continuum of the spectra, $H$ is the intrinsic line profile, $G$ is the rotational profile. In other words, the line profile of a rotating star is simply the convolution between the line profile without rotation with the rotational profile.

$G$ can be evaluated by assuming the linear limb darkening law in eq. 17.11 from Gray (2005), which gives

$$
G(v)=\frac{2(1-\epsilon)\left[1-\left(v / v_{\mathrm{L}}\right)^{2}\right]^{1 / 2}+\frac{1}{2} \pi \epsilon\left[1-\left(v / v_{\mathrm{L}}\right)^{2}\right]}{\pi v_{\mathrm{L}}(1-\epsilon / 3)},
$$

where $v_{\mathrm{L}}$ is the projected rotational velocity and $\epsilon$ is the limb darkening coefficient. Fig. 1.5 shows a plot of the rotational profile using $\epsilon=0.6$. The first term of Eq. 1.15 corresponds to an ellipse, while the second one corresponds to a parabola.

As we will see in Section 3.4.2, eq. 1.15 is crucially important in the estimation of the projected rotational velocities of the solar twins, because it allows us to precisely model the contribution of spectral line broadening by rotation using extremely high-resolution spectra. 


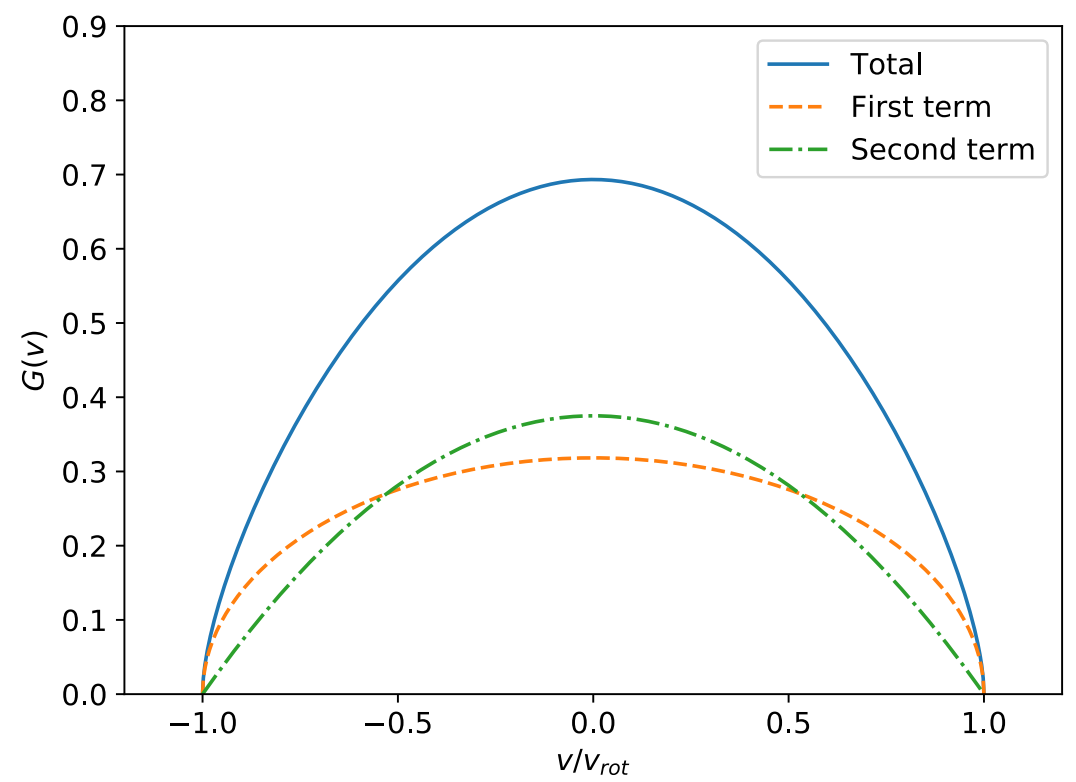

Figure 1.5: The rotational profile as described by Eq. 1.15, using a limb darkening coefficient $\epsilon=0.6$. 
Chapter 2

\section{The data and observational constraints}

The data we used in this study comes mainly from our HARPS Large Program "The Solar Twin Planet Search,"but the discussion and interpretation of the results also depend on data obtained with the MIKE spectrograph and information derived from them (i.e., the observational constraints). Additional radial velocity data was obtained from the literature and online archives of several instruments and programs. We will discuss below the most important aspects of these items.

\subsection{Data from the MIKE spectrograph}

The original sample of this study was comprised of 88 solar twins, compiled by Ramírez et al. (2014, and references therein). The stars were observed between January 2011 and May 2012 at the $6.5 \mathrm{~m}$ Clay Magellan Telescope, located at the Las Campanas Observatory, Chile, using the MIKE spectrograph (Bernstein et al., 2003).

The exposure times and configurations of the instrument are thoroughly described by Ramírez et al. (2014), as well as the data reduction process. The wavelength coverage was $320-1000$ $\mathrm{nm}$, with a $0.35^{\prime \prime}$ slit width, which resulted in a nominal resolving power $R=\lambda / \Delta \lambda=83000$ in the blue arm and 65000 in the red arm. The signal-to-noise ratio $(S / N)$ of the observations was approximately 400 per pixel. According to the MIKE User Manual ${ }^{1}$, the slit-to-detector efficiencies are roughly $37 \%$ on $4500 \AA$ and $20 \%$ on $6500 \AA$. See more technical specifications of the MIKE spectrograph in Table 2.1.

A careful analysis of the MIKE data revealed that most observations had resolutions in the red arm that were significantly below the nominal, varying between 40000 and 50000, particu-

\footnotetext{
${ }^{1}$ Available at: http://www. lco.cl/Members/magins/mike-kb/mike-user-manual
} 
Table 2.1 - Technical specifications of the MIKE spectrograph. Numbers are approximate (source: MIKE User Manual).

\begin{tabular}{l|c|c|l}
\hline & Blue arm & Red arm & Unit \\
\hline Effective focal ratio & $\mathrm{f} / 3.9$ & $\mathrm{f} / 3.6$ & \\
Detector size & $2048 \times 4096$ & $2048 \times 4096$ & $\mathrm{px} \times \mathrm{px}$ \\
Wavelength scale & 0.02 & 0.05 & $\AA \mathrm{px}^{-1}$ \\
Plate scale & 8.2 & 7.5 & $\mathrm{px} \mathrm{arcsec}^{-1}$ \\
Read noise & 2.0 & 3.5 & $\mathrm{e}^{-} \mathrm{px}^{-1}$ \\
Gain & 0.47 & 1.0 & $\mathrm{e}^{-} \mathrm{ADU}^{-1}$ \\
Dark current & 5 & 2 & $\mathrm{ADU} \mathrm{px}^{-1} \mathrm{~h}^{-1}$ \\
Wavelength range & $3200-5000$ & $4900-10000$ & $\AA$ \\
Resolving power & 83000 & 65000 & \\
\hline
\end{tabular}

larly in the first two observation runs (January and June 2011; see Fig. 2.1); the subsequent three runs (September 2011, February and May 2012) had more stable resolving power values varying between 60000 and 65000. Since the solar twins have very low rotational velocities (up to $\sim 4$ $\mathrm{km} \mathrm{s}^{-1}$ ), the spectral line broadening is dominated by the instrumental contribution, and the instability of resolutions can introduce significant uncertainties on our measurements of rotational velocities. Owing to this issue, in Chapter 3 we decided to use our data obtained at the HARPS spectrograph, which has a stable resolving power of $R=115000$ in the red arm.

Nevertheless, the MIKE data was used to estimate the stellar parameters due to their prompt availability and high $S / N$. For the differential approach applied in this estimation, the spectral resolution is an important issue, but not the main source of uncertainties - which can be mainly attributed to measurement errors of the equivalent widths of the spectra and uncertainties in the applied stellar atmosphere models -, unlike the estimation of rotational velocities. A complete discussion on the estimates and uncertainties of the stellar parameters is available in Ramírez et al. (2014).

It is also important to note that the solar twins in our program are very bright and are thus not photon starved in either instrument. The stellar parameters of the solar twins sample are currently being updated using the HARPS spectra (Spina et al. in preparation). 

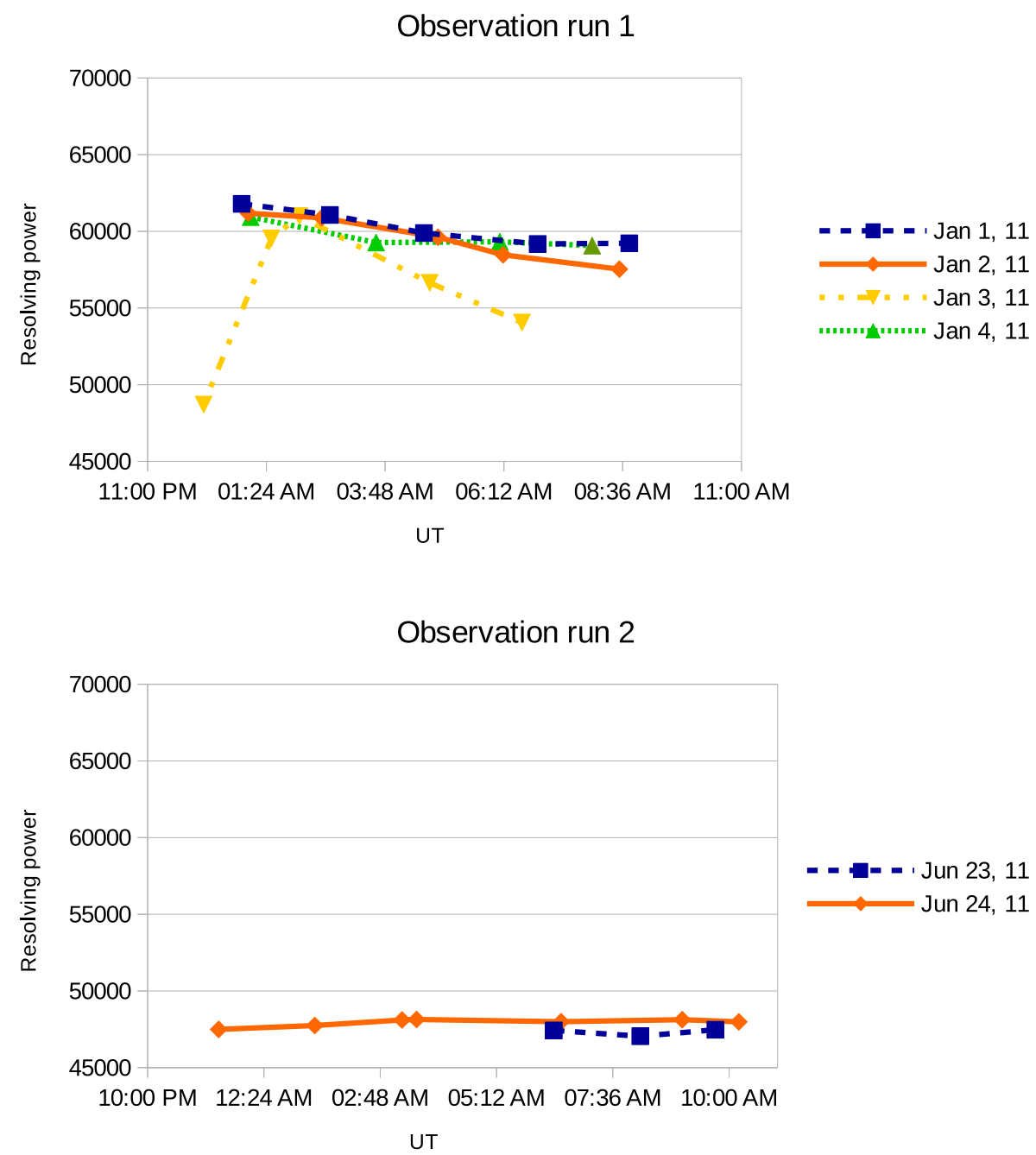

Figure 2.1: Variation of the MIKE spectral resolving power in the red arm as a function of observing time (UT) during two observation runs.

\subsection{Data from the HARPS spectrograph}

HARPS is an extremely stable spectrograph installed in an evacuated and temperature controlled vacuum enclosure (Mayor et al., 2003), which is optimized for very precise measurements of radial velocities aiming to detect extrasolar planets. It is fed by ESO's $3.6 \mathrm{~m}$ telescope at La Silla Observatory. Our observations come from The Solar Twin Planet Search large program (ID 188.C-0265, PI: Jorge Meléndez), which looked for exoplanets orbiting stars similar to the Sun (Meléndez et al., 2015).

In total, we obtained high-resolution $\left(R>10^{5}\right)$ and high signal to noise $(S / N>500)$ spectra for 81 stars, all of them overlapping the 88 solar twins from Ramírez et al. (2014). We used the solar spectrum (light reflected from asteroid Vesta) described by Nissen (2015), which was 
Table 2.2 - Technical specifications of the HARPS spectrograph (source: HARPS User Manual).

\begin{tabular}{ll}
\hline Camera focal ratio & $\mathrm{f} / 3.3$ \\
Detector size & $2 \times 2048 \times 4096 \mathrm{px}^{2}$ \\
Sampling & $3.4 \mathrm{px} \mathrm{FWHM}^{-1}$ \\
Read noise & $\sim 2.9 \mathrm{e}^{-} \mathrm{px}^{-1}$ \\
Gain & $2.04 \mathrm{e}^{-} \mathrm{ADU}^{-1}$ \\
Dark current & not measured \\
Wavelength range & $3780-6910 \AA$ \\
Resolving power & 115000 \\
\hline
\end{tabular}

obtained with the same instruments and configurations as our solar twins.

Outside our HARPS large program, we also used data from the following ESO programs: 183.D-0729, 292.C-5004, 077.C-0364, 072.C-0488, 092.C-0721, 093.C-0409, 183.C-0972, 192.C0852, 091.C-0936, 089.C-0732, 091.C-0034, 076.C-0155, 185.D-0056, 074.C-0364, 075.C0332, 089.C-0415, 60.A-9036, 075.C-0202, 192.C-0224, 090.C-0421 and 088.C-0323.

The processing of the spectra was automatically applied on-site with the HARPS Data Reduction Software (DRS). The DRS applies Python scripts and it is not openly available online.

According to the DRS User Manual ${ }^{2}$, the raw spectra are pre-processed with a simple bias subtraction and correction of bad columns, followed by a dark correction. The spectral orders are located using a threshold condition (normalized central row value above 0.15) and then traced at 20 pixel steps, and the geometry of orders is fitted with 4th order polynomials. The flat field spectra (tungsten exposures) are used to compute the pseudo-blaze response of each order.

The spectral extraction applied by the DRS is based on the optimal extraction algorithm by Horne (1986), which differs from the standard extraction by applying decreasing weigths for the pixels farther away from the spectral cross-section center before collapsing them in the crosssection direction. Furthermore, the optimal extraction assumes the cross-section profiles are perfectly aligned with the CCD columns or, in other words, the weights of the optimal extraction are such that $w=w(x, \lambda)$, where $x$ is a given column of the CCD and $\lambda$ is the wavelength.

Recent studies have proposed more robust spectral extraction algorithms (e.g., Piskunov and Valenti, 2002; Bolton and Schlegel, 2010; Zechmeister et al., 2014), but it is not clear how much improvement they would provide to the spectra obtained with HARPS. A quantitative analysis of the expected improvement is beyond the scope of this study, but we expect that these

\footnotetext{
${ }^{2}$ Available at http://wWw. eso.org/sci/facilities/lasilla/instruments/harps/doc.html
} 
more complex extraction algorithms may be required for the new generation of planet-hunting spectrographs, such as MAROON-X (Seifahrt et al., 2016), EXPRES (Jurgenson et al., 2016) and G-CLEF (Szentgyorgyi et al., 2012), especially when targeting rocky planets.

Wavelength calibration is performed by fitting the observed spectrum of a thorium lamp to the laboratory template spectrum and applying the resulting calibration to the science spectra, which are then corrected for the barycentric Earth radial velocity. The radial velocities (RVs) of the target star are measured by cross-correlating the science spectra with a G2-type mask.

The contribution from photon noise to the uncertainties of the HARPS radial velocities notice that it is different from the rotational velocity - is inversely proportional to the $S / N$ of the spectra. The telescope focus, centering and wavelength calibration errors contribute with 0.3 (upper limit), 0.2 and $0.5 \mathrm{~m} \mathrm{~s}^{-1}$, respectively. The baseline uncertainty for HARPS RVs is approximately $1 \mathrm{~m} \mathrm{~s}^{-1}$, which is only achieved for $S / N>100$ per pixel at $550 \mathrm{~nm}$.

More about the post-processing we performed on the HARPS data can be found in Section 3.3 .

\subsection{Other programs}

As we will see in Chapter 4, we needed additional data from other programs to broaden our coverage of our radial velocities of the binaries in the solar twin sample. We obtained more RV datasets that were available in the literature and in public databases.

\subsubsection{Data from the literature}

For this work, we used extra RV data from Willmarth et al. (2016) for the star HIP 67620, obtained at the Kitt Peak 0.9 m auxiliary feed telescope equipped with a coudé spectrograph and from the echelle spectrograph at the Fairborn Observatory. The RVs were measured by crosscorrelating stellar spectra with the solar spectrum (sky). Additionally, we used archival RV data for HIP 65708 obtained with the CfA Digital Speedometers (Latham et al., 2002) and measured with one-dimensional correlation of the observed spectra with templates (uncertainties in the order of $1 \mathrm{~km} \mathrm{~s}^{-1}$ ). 


\subsubsection{Data from the ELODIE and SOPHIE spectrographs}

We also obtained extra RV data from the archives of ELODIE, the cross-dispersed échelle spectrograph fed by the $1.93 \mathrm{~m}$ telescope at L'Observatoire de Haute-Provence (Baranne et al., 1996) for the stars HIP 43297, 54582, 62039, 64150, 72043, and 87769. The reported performance of ELODIE for RV precision sits at an instrumental uncertainty of $0.013 \mathrm{~km} \mathrm{~s}^{-1}$. The radial velocities were computed with an optical cross-correlation process described on Baranne et al. (1979); Queloz (1995), using standard masks of an F0V- and a K0III-type star.

Additionally, we included archival RV data from SOPHIE, the successor to ELODIE, installed at the same telescope (Perruchot et al., 2011), for the stars HIP 6407, 43297, 54582, 62039, 64150, and 87769. The data reduction follows a similar approach adopted for the HARPS spectrograph data, and is described in the instrument's website ${ }^{3}$. The RVs were obtained by a crosscorrelation analysis using the mask of a G2-type star. The stability of the radial velocities for this instrument varies from 3 to $4 \mathrm{~m} \mathrm{~s}^{-1}$ over several months.

\subsubsection{Data from the HIRES spectrograph}

Extra RV data for this work were obtained with HIRES ${ }^{4}$ (Vogt et al., 1994; Butler et al., 2017), an échelle cross-dispersed spectrograph which is fed by the $10 \mathrm{~m}$ Keck telescope. The stars with HIRES data are HIP 19911, 62039, 64150, 72043, and 103983.

\subsubsection{Data from the Lick Planet Search}

The Lick Planet Search was a program that initially aimed to observe 120 bright FGK stars and M dwarfs with the Hamilton Spectrograph échelle spectrometer fed by the coudé focus of the $3 \mathrm{~m}$ Shane Telescope, located at the Lick Observatory. It obtained radial velocity data for two stars in our sample: HIP 54582 and 65708. The data is fully described and publicly available on Fischer et al. (2014, and references therein).

\subsubsection{Data from the Anglo-Australian Telescope Planet Search}

Jenkins et al. (2015) present an analysis of the binary stars on the sample of the AngloAustralian Planet Search program, which overlap our sample with the stars HIP 67620, 73241,

\footnotetext{
${ }^{3}$ http://www.obs-hp.fr/guide/sophie/data_products.shtml

${ }^{4}$ Data publicly available at http://home.dtm.ciw.edu/ebps/data/
} 
79578 and 81746. They were observed with the UCLES spectrograph at the Anglo-Australian Telescope. In their study, they also include the HARPS data that were publicly available at the time, and the orbital parameters they obtain are presented on Table 2 and Figure 1 in Jenkins et al. (2015), along with the RV data on Table 3 of the same paper.

\subsection{The isochronal ages and masses of the solar twin sample}

The main points of this research are to assess if the Sun is a regular rotator for its age and to study the rotational evolution of Sun-like stars using a well-defined sample of stars strictly similar to the Sun. These aims can only be achieved if we have a reliable estimation of the ages of these stars. As mentioned in Section 1.3, there are several ways to obtain these age estimates, with gyrochronology being considered one of the most reliable ones, at least for young stars.

For this study, we cannot use rotational ages because it would result in circular reasoning - in other words, we would be using rotation to measure ages, which are then used to assess the evolution of rotation with age. Moreover, there is a prior knowledge that our sample of stars comprises a large range of ages varying from 1 to 10 Gyr (Ramírez et al., 2014). Another problem for gyrochronology is that stars that somehow had their angular momentum history changed by interaction with a companion will not have reliable rotational ages.

Asteroseismic ages are not available for all the stars in the sample, so we cannot consistently use them in this study. On the other hand, age estimates using lithium decay are an alternative option, but the physics behind lithium depletion is still poorly understood, and there is considerable debate in the literature about the precision and accuracy of the method, even for solar-type stars (see, e.g., Jeffries, 2014).

Given the aforementioned caveats, we decided to use isochronal ages for the solar twin sample. They were determined by Tucci Maia et al. (2016) using the Yonsei-Yale isochrones (also known as $\mathrm{Y}^{2}$; Yi et al., 2001). The age estimation is performed as described by Ramírez et al. (2013, 2014), and the process is outlined below.

\subsubsection{Computing probability distributions}

Isochrones are, by definition, the locus in a Hertzprung-Russel (HR) diagram where a given set of stellar evolution tracks have the same age (see Fig. 2.2). Each isochrone point is represented by the set of parameters $(x, t, r, f)$, where $x$ is the stellar age or mass, $t$ is the effective 
temperature, $r$ is the surface gravity and $f$ is the iron abundance of the model. The probability that this point corresponds to a given set of observational constraints is described by

$$
p(x, t, r, f) \propto \exp \left[-\frac{\left(T_{\mathrm{eff}}-t\right)^{2}}{\left(\sqrt{2} \sigma_{T_{\mathrm{eff}}}\right)^{2}}\right] \times \exp \left[-\frac{(\log g-\log r)^{2}}{\left(\sqrt{2} \sigma_{\log g}\right)^{2}}\right] \times \exp \left[-\frac{([\mathrm{Fe} / \mathrm{H}]-f)^{2}}{\left(\sqrt{2} \sigma_{[\mathrm{Fe} / \mathrm{H}]}\right)^{2}}\right]
$$

where $\sigma$ corresponds to the uncertainties of the observational constraints $T_{\text {eff }}$ (effective temperature), $\log g$ (surface gravity) and $[\mathrm{Fe} / \mathrm{H}]$ (iron abundance).

The stellar parameters were computed in a line-by-line differential approach, which leverages the extreme similarity of the solar twins with the Sun - a well known star - and the high $S / N$ of the MIKE spectra to produce very precise and accurate estimates for $T_{\text {eff }}, \log g$ and $[\mathrm{Fe} / \mathrm{H}]$. The uncertainties are, on average, $\sigma_{T_{\text {eff }}}= \pm 7 \mathrm{~K}, \sigma_{\log g}= \pm 0.019 \operatorname{dex}(\mathrm{cgs})$ and $\sigma_{[\mathrm{Fe} / \mathrm{H}]}= \pm 0.006 \mathrm{dex}$ (Ramírez et al., 2014).

The probability distribution of $x$ is then computed as

$$
P(x)=\sum_{x^{\prime}=x-\delta x}^{x^{\prime}=x+\delta x} p\left(x^{\prime}, t, m, f\right),
$$

where $\delta x$ is a small step in $x$ and the sum encompasses radii of three times the uncertainties of the observational constraints. The most likely value for $x$ is given by the center of the bin corresponding to the maximum value of $P(x)$. The $1 \sigma$ uncertainties of $x$ are given by the $68 \%$ confidence limit of the estimate.

\subsubsection{Concerns in the use of isochronal ages}

As pointed out by Brown (2014); Ramírez et al. (2013, and references therein), using this probabilistic approach may overestimate the stellar ages, and these biases are important for absolute ages, which is the case of this study. On the other hand, Chanamé and Ramírez (2012) showed that the results obtained with this method are in good agreement with the ones estimated with Bayesian approaches (which aim to minimize such biases).

For the solar twin HIP 64150, which has a white dwarf (WD) binary companion, Matthews et al. (2014) estimated a WD cooling age of $7.77 \pm 0.24$ Gyr using a best fit hydrogen model (Crepp et al., 2013; Bergeron et al., 2011). This value is in decent agreement with the isochronal age estimate using the MIKE spectra, which was $6.41 \pm 0.66$ Gyr (see Section 3.6). Notice that an even better WD cooling age could be obtained if the stellar parameters used for the main star 


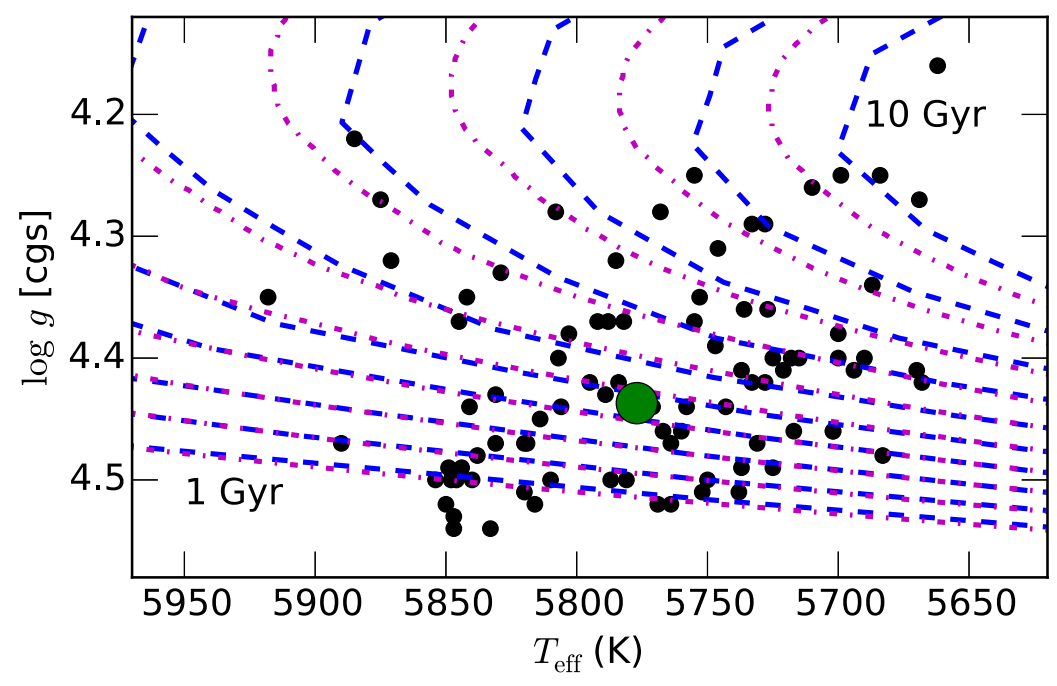

Figure 2.2: Isochronal age estimates for the stars in the Solar Twin Planet Search (dark circles) and the Sun (green circle). Dashed and dot-dashed curves correspond to Yonsei-Yale and Darmouth isochrones, respectively (Tucci Maia et al., 2016).

had the same precision as the one we report for this solar twin. Naturally, this field of research would benefit immensely from the identification of these Sirius-like systems and the estimation of their WD cooling ages to be compared with other methods.

Another common criticism to the isochrones method is related to the stellar evolution models. In that regard, Stancliffe et al. (2016) provides a detailed analysis on the differences between various stellar evolution tracks available in the literature (including the $\mathrm{Y}^{2}$ models), as well as their deficiencies when we try to reconcile the most modern observational constraints of the Sun (e.g., Rhodes et al., 1997; Bahcall et al., 2001; Asplund et al., 2009) with the free parameters of the models. Some of the most important considerations and results obtained by Stancliffe et al. (2016) for Sun-like stars are as follows:

1. If the Sun was observed as a star using Gaia (which has uncertainties of $\pm 70 \mathrm{~K}$ in $T_{\mathrm{eff}}$ and \pm 0.05 mag in bolometric correction), the spread in its estimated mass based on the results from different stellar evolution tracks would be approximately $\pm 0.02 \mathrm{M}_{\odot}$.

2. A $10 \%$ error in the input metallicity $Z$ produces an error of $2 \%$ in the stellar mass determination.

3. The luminosities of different models vary by only $\sim 0.05 \%$ for a $1 \%$ change in the mixing length parameter. 
4. Differences in boundary conditions do not produce significative changes in stellar evolution tracks in the main sequence.

When modelling stellar evolution, part of the input physics, such as the mixing length parameter, are fine-tuned to match the observed parameters of the Sun (see Table 1 in Yi et al., 2001), in a process known as solar calibration. Other parameters, such as the convective core overshooting are also tested mainly for solar metallicity. In relation to the microscopic physics, Stancliffe et al. (2016) explains that the reaction rates are of least concern as a source of errors, and different choices of opacities only affect stars in the red giant phase or very low-mass stars in the beginning of the main-sequence.

Following the work of Meléndez et al. (2012), Ramírez et al. (2014) and Tucci Maia et al. (2016) calibrate the isochrones with a shift in metallicity of -0.04 dex so that the $\mathrm{Y}^{2}$ models reproduce the solar mass and age. Dotter et al. (2017) point out that this calibration is, in effect, a correction for radiative diffusion in the surface chemical abundances of the Sun, a process that is not considered directly by the $\mathrm{Y}^{2}$ models.

The fact that we use strictly solar twins in the MS and differential analysis, coupled with the aforementioned precautions and considerations gives us confidence about the results for solar twins that have approximately the same age as the Sun.

For younger solar twins, the isochrones are more tightly packed, which is why the resulting estimates tend to have larger uncertainties. On the other hand, because the isochrones are close to the Sun, we expect that their accuracy is higher. For the older solar twins, the isochrones are more sparsely located, which results in lower uncertainties. However, due to their distance from the solar-age isochrone, it is expected that their accuracy is less reliable if we want to adopt a more conservative position. On the other hand, Tucci Maia et al. (2016) found that the isochronal age of the old solar twin 16 Cyg is in excellent agreement with the asteroseismic age estimated by van Saders et al. (2016).

As pointed out by Meléndez et al. (2012, Appendix D), the high precision of the stellar parameters obtained with differential analysis of solar twins does not allow for arbitrary values of He abundance. Meléndez et al. found that, when fixing the mass of HIP 56948 to its best value $\left(1.012 \mathrm{M}_{\odot}\right)$, the uncertainties of $\log g$ only allow values of $\mathrm{He}$ abundances within $\pm 1 \%$ of the solar value, and that the stellar age is insensitive of the adopted He content.

It is also important to note that the $\mathrm{Y}^{2}$ models do not employ non-standard physics (see, e.g., do Nascimento et al., 2009; Li et al., 2012, and references therein). On that regard, Yana 
Galarza (2016) tested the use of non-standard physics for the solar twin 18 Sco (HIP 79672) and concluded that they do not produce significant changes in the estimate of stellar age for that star when using a differential analysis. Future studies in this line of research should test and use the more recent Yale-Postdam isochrones (Spada et al., 2017), which apply more modern techniques, as well as the updated OP opacities (Badnell et al., 2005). 
Chapter 3

\section{Rotational evolution of Sun-like stars}

This chapter is based on the article published in dos Santos et al. (2016). The co-authors J. Meléndez, J.-D. do Nascimento, M. Bedell, I. Ramírez, J. L. Bean, M. Asplund, L. Spina, S. Dreizler, A. Alves-Brito and L. Casagrande contributed with the observations, data reduction, data analysis and discussion of the results. A copy of the publication can be found in Appendix B.

\subsection{Abstract of this chapter}

It is still unclear how common the Sun is when compared to other similar stars in regards to some of its physical properties, such as rotation. Considering that gyrochronology relations are widely used today to estimate ages of stars in the main sequence, and that the Sun is used to calibrate it, it is crucial to assess whether these procedures are acceptable. We analyze the rotational velocities, limited by the unknown rotation axis inclination angle, of an unprecedented large sample of solar twins to study the rotational evolution of Sun-like stars, and assess whether the Sun is a typical rotator. We used high-resolution $(R=115000)$ spectra obtained with the HARPS spectrograph and the $3.6 \mathrm{~m}$ telescope at La Silla Observatory. The projected rotational velocities for 81 solar twins were estimated by line profile fitting with synthetic spectra. Macroturbulence velocities were inferred from a prescription that accurately reflects their dependence with effective temperature and luminosity of the stars. The sample of solar twins includes some spectroscopic binaries with enhanced rotational velocities. We verified that the Sun does not have a peculiar rotation, but the solar twins exhibit rotational velocities that depart from the Skumanich relation. The Sun is a regular rotator when compared to solar twins with a similar age. Additionally, we obtain a rotational braking law that better describes the stars in the 
sample $\left(v \propto t^{-0.6}\right)$ in contrast to previous, often-used scalings.

\subsection{The role of the Sun in our understanding of rotational evolution}

The Sun is the best-known star to astronomers and is commonly used as a template in the study of other similar objects. Yet, there are still some of its aspects that are not well understood and that are crucial for a better understanding of how stars and, consequently, how planetary systems and life evolve: How do the more complex physical parameters of a Sun-like star, such as rotation and magnetic activity, change with time? Is the Sun unique or typical (i.e., an average Sun-like star)? If the Sun is common, it would mean that life does not require a special star for it to flourish, eliminating the need to evoke an anthropic reasoning to explain it.

In an effort to assess how typical the Sun is, Robles et al. (2008) compared 11 of its physical parameters with nearby stars, and concluded that the Sun is, in general, typical. Although they found it to be a slow-rotator against $276 \mathrm{~F} 8-\mathrm{K} 2$ (within $\pm 0.1 \mathrm{M}_{\odot}$ ) nearby stars, this result may be rendered inconclusive owing to unnacounted for noise that is caused by different masses and ages in their sample. Other studies have suggested that the Sun rotates either unusually slowly (Smith, 1979; Leão et al., 2015) or regularly for its age (Soderblom, 1983, 1985; Gray, 1984; Gustafsson, 1998; Barnes, 2003), but none of these investigations comprised stars that are very similar to the Sun, therefore preventing a reliable comparison. In fact, with Kepler and CoRoT, it is now possible to obtain precise measurements of rotation periods, masses and ages of stars in a very homogeneous way (e.g., Ceillier et al., 2015; do Nascimento et al., 2012; Chaplin et al., 2014), but they generally lack high-precision stellar parameters, which are accessible through spectroscopy. The challenging nature of these observations limited ground-based efforts to smaller, but key stellar samples (e.g., Pizzolato et al., 2003; Strassmeier et al., 2012).

The rotational evolution of a star plays a crucial role in stellar interior physics and habitability. Previous studies proposed that rotation can produce extra mixing that is responsible for depleting the light elements Li and Be in their atmospheres (Pinsonneault et al., 1989; Charbonnel et al., 1994; Tucci Maia et al., 2015), which could explain the disconnection between meteoritic and solar abundances of $\mathrm{Li}$ (Baumann et al., 2010). Moreover, rotation is highly correlated with magnetic activity (e.g., Noyes et al., 1984; Soderblom et al., 1993; Baliunas et al., 1995; Mamajek and Hillenbrand, 2008) and magnetic fields (Vidotto et al., 2014; Marsden et al., 2014); these trends are key to understanding how planetary systems and life evolve in face of varying 
magnetic activity and energy outputs by solar-like stars during the main sequence (Guinan and Engle, 2009; Ribas et al., 2005; do Nascimento et al., 2016).

One of the pioneer efforts in empirical studies of rotational evolution produced the wellknown Skumanich relation $v \propto t^{-1 / 2}$ (see Section 1.3), which describes the rotational evolution of solar-type stars in the main sequence, and can be derived from the loss of angular momentum due to magnetized stellar winds (see Section 1.2 and, e.g., Kawaler, 1988; Charbonneau, 1992; Barnes, 2003; Gallet and Bouvier, 2013). This relation sparked the development of gyrochronology, which consists in estimating stellar ages based on their rotation, and it was shown to provide a stellar clock as good as chromospheric ages (Barnes, 2007). In Skumanich-like relations, however, the Sun generally falls on the curve (or plane, if we consider dependence on mass) defined by the rotational braking law by design. Thus it is of utmost importance to assess how common the Sun is to correctly calibrate it.

Subsequent studies have proposed modifications to this paradigm of rotation and chromospheric activity evolution (e.g., Soderblom et al., 1991; Pace and Pasquini, 2004), exploring rotational braking laws of the form $v \propto t^{-b}$. The formalism by Kawaler (1988) shows that this index $b$ can be related to the geometry of the stellar magnetic field, and that the Skumanich index $(b=1 / 2)$ corresponds to a geometry that is slightly more complex than a simple radial field. Such prescriptions for rotational evolution have a general agreement for young ages up to the solar age (see Sood et al., 2016; Amard et al., 2016, and references therein), but the evolution for older ages still poses an open question. In particular, van Saders et al. (2016) suggested that stars undergo a weakened magnetic braking after they reach a critical value of the Rossby number, thus explaining the stagnation trend observed on the rotational periods of older Kepler stars ${ }^{1}$.

In order to assess how typical the Sun is in its rotation, this study aims to verify whether the Sun follows the rotational evolution of stars that are very similar to it, which is an objective that is achieved by precisely measuring their rotational velocities and ages. We take advantage of an unprecedented large sample of solar twins, which provides us with precise stellar parameters and is essential for the analysis that we perform (see Fig. 3.1 for an illustration of the subtle effects of rotation in stellar spectra of Sun-like stars).

\footnotetext{
${ }^{1}$ Notice, however, that their stars are not necessarily solar twins or analogs, as their stellar parameters are uncertain.
} 


\subsection{Working sample}

The sample consists of bright solar twins (see Section 1.1.1 for the definition) in the Southern Hemisphere, which were mostly observed in our HARPS Large Program (ID: 188.C-0265) at the European Southern Observatory (ESO) that aimed to search for planetary systems around stars very similar to the Sun (Ramírez et al., 2014; Bedell et al., 2015; Tucci Maia et al., 2016, which, in this chapter, we will refer to as Papers I, II and III, respectively, of the series The Solar Twin Planet Search). In total, we obtained high-precision spectra for 73 stars and used data from 9 more targets observed in other programs; all of these overlapped the sample of 88 stars from Paper I. We used the spectrum of the Sun (reflected light from the Vesta asteroid) from the ESO program 088.C-0323, which was obtained with the same instrument and configuration as the solar twins. See a more detailed description of the HARPS data, as well as its reduction, in Section 2.2.

The ages of the solar twin sample span between $0-10$ Gyr and are presented in Table A.1. They were obtained in Paper III using Yonsei-Yale isochrones (see Section 2.4 for a more detailed description), and the uncertainties were assumed to be symmetric. These ages are in excellent agreement with those obtained in Paper I, with a mean difference of $-0.1 \pm 0.2 \mathrm{Gyr}$ (see footnote 5 in Paper III). We adopted 4.56 Gyr for the solar age (Bahcall et al., 1995). The other stellar parameters $\left(T_{\text {eff }}, \log g,[\mathrm{Fe} / \mathrm{H}]\right.$ and microturbulence velocities $\left.v_{\mathrm{t}}\right)$ were obtained in Paper I. The stellar parameters of HIP 68468 and HIP 108158 were updated in Paper III (see Appendix A).

For the rotational analysis, we did not use observations for 18 Sco (HIP 79672) from May 2009, owing to their instrumental artifacts, and we did not include observations post-HARPS upgrade (June 2015) when combining the spectra - they had a different shape in the red side, and since there were few observations, we chose not to use them to eliminate eventual problems with combination and normalization. The initial plan was to use the observations from the MIKE spectrograph, as described in in Section 2.1. However, we decided to use the HARPS spectra due to its higher spectral resolving power.

The wavelength coverage for the observations ranges from 3780 to $6910 \AA$, with a spectral resolving power of $R=\lambda / \Delta \lambda=115000$. Each spectrum was divided into two halves, corresponding to the mosaic of two detectors (one optimized for blue and the other for red wavelengths). In this analysis we only worked with the red part (from 5330 to $6910 \AA$ ) because of its higher $S / N$ and the presence of cleaner lines. The correction for radial velocities was performed with the 


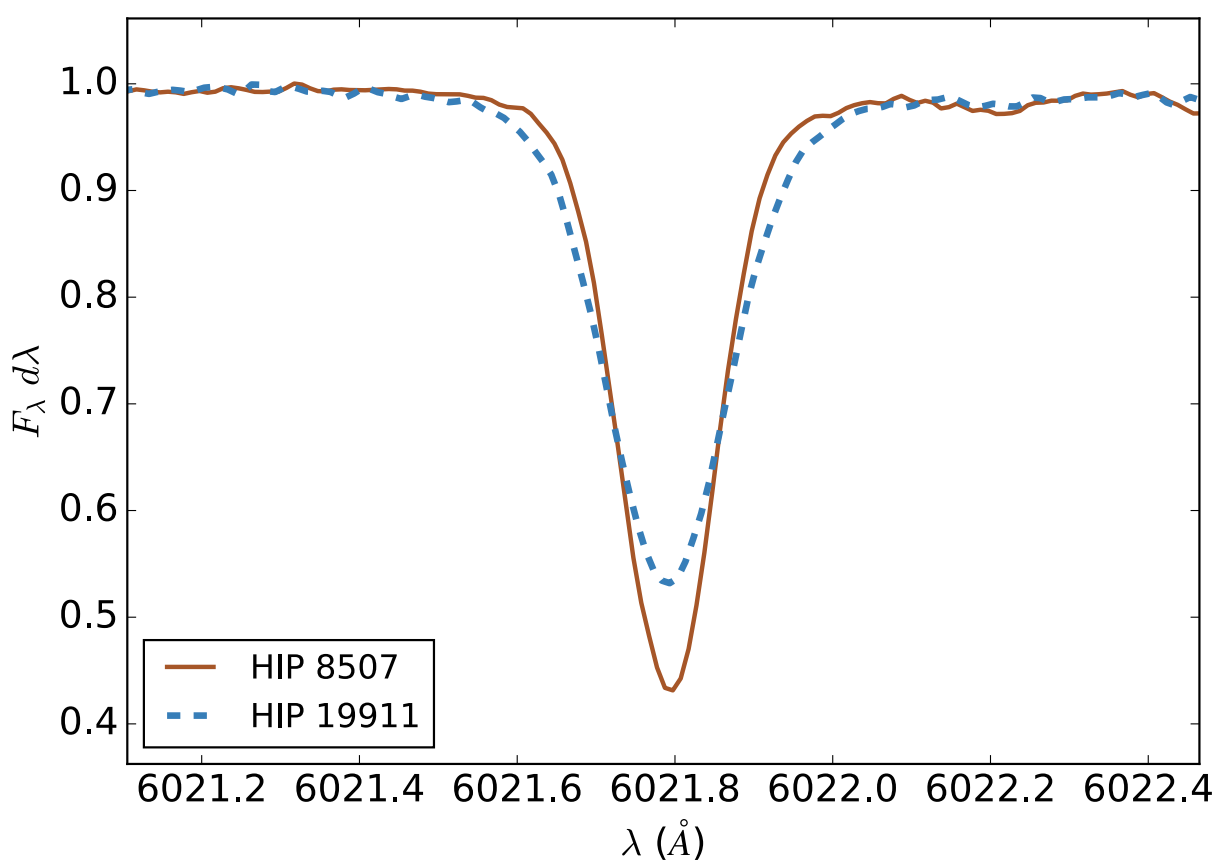

Figure 3.1: Comparison of the spectral line broadening between two solar twins with different projected rotational velocities. The wider line corresponds to HIP 19911, with $v \sin i \approx 4.1 \mathrm{~km} \mathrm{~s}^{-1}$, and the narrower line comes from HIP 8507, with $v \sin i \approx 0.8 \mathrm{~km} \mathrm{~s}^{-1}$.

task dopcor from $\mathrm{IRAF}^{2}$, using the values obtained from the cross-correlation function (CCF) of the HARPS pipeline. The different observations were combined with IRAF's scombine task. The resulting average (of the sample) signal to noise ratio was 500 around $6070 \AA$. The red regions of the spectra were normalized with 30th order polynomial fits to the upper envelopes of the entire red range, using the task continuum on IRAF. Care was taken so that the continuum of the stars were consistent with the Sun's. Additionally, we verified that errors in the continuum determination introduce uncertainties in $v \sin i$ lower than $0.1 \mathrm{~km} \mathrm{~s}^{-1}$.

\subsection{Methods}

We analyzed five spectral lines, four due to Fe I and one to Ni I (see Table 3.1; equivalent widths were measured using the task splot in IRAF), which were selected for having low levels of contamination by blending lines. The rotational velocity of a star can be measured by estimating the spectral line broadening that is due to rotation. The rotation axes of the stars are

\footnotetext{
${ }^{2}$ IRAF is distributed by the National Optical Astronomy Observatories, which are operated by the Association of Universities for Research in Astronomy, Inc., under cooperative agreement with the National Science Foundation.
} 
randomly oriented, thus the spectroscopic measurements of rotational velocity are a function of the inclination angle $(v \sin i)$.

Table 3.1 - Line list used in the projected stellar rotation measurements. EW are the equivalent widths and $v_{\text {macro }}$ are the macroturbulence velocities measured as in Sect. 3.4.1.

\begin{tabular}{cccccc}
\hline $\begin{array}{c}\text { Wavelength } \\
(\AA)\end{array}$ & $\mathrm{Z}$ & $\begin{array}{c}\text { Exc. pot. } \\
(\mathrm{eV})\end{array}$ & $\log (g f)$ & $\begin{array}{c}v_{\text {macro }}^{\odot} \\
\left(\mathrm{km} \mathrm{s}^{-1}\right)\end{array}$ & $\begin{array}{c}\mathrm{EW}^{\odot} \\
(\AA)\end{array}$ \\
\hline 6027.050 & 26 & 4.076 & -1.09 & 3.0 & 0.064 \\
6151.618 & 26 & 2.176 & -3.30 & 3.2 & 0.051 \\
6165.360 & 26 & 4.143 & -1.46 & 3.1 & 0.045 \\
6705.102 & 26 & 4.607 & -0.98 & 3.6 & 0.047 \\
6767.772 & 28 & 1.826 & -2.17 & 2.9 & 0.079 \\
\hline
\end{tabular}

We estimate $v \sin i$ for the sample of solar twins using the 2014 version of MOOG synth (Sneden, 1973), adopting stellar atmosphere models by Castelli and Kurucz (2004), with interpolations between models performed automatically by the Python package qoyllur-quipu ${ }^{3}$ (see Paper I). The instrumental broadening is taken into account by the spectral synthesis. We used the stellar parameters from Paper III and microturbulence velocities from Paper I. Macroturbulence velocities $\left(v_{\text {macro }}\right)$ were calculated by scaling the solar values, line by line (see Sect. 3.4.1). The estimation of the rotational velocities was performed with our own algorithm ${ }^{4}$ that makes automatic measurements for all spectral lines for each star. We applied heuristic fine-tuning corrections for the nonsatisfactory automatic line profile fittings, and quote $v \sin i$ as the mean of the values measured for the five lines. See Sections 3.4.1 and 3.4.2 for a detailed description on rotational velocities estimation and their uncertainties. In Fig. 3.2, which shows an example of spectral line fitting for one feature in the Sun, stellar granulation produces the slight asymmetry present in the observed spectrum. This effect is not modelled in MOOG, but it is well-documented and arises from the velocity fields in stellar photospheres (see Chapter 17 in Gray, 2005, and references therein).

\subsubsection{Macroturbulence velocities}

We tested the possibility of measuring $v_{\text {macro }}$ (radial-tangential profile) simultaneously with $v \sin i$, but even when using the extremely high-resolution spectra of HARPS, it is difficult to

\footnotetext{
${ }^{3}$ Available at https://github.com/astroChasqui/q2

${ }^{4}$ Available at https://github.com/RogueAstro/PoWeRS, currently not being maintained.
} 


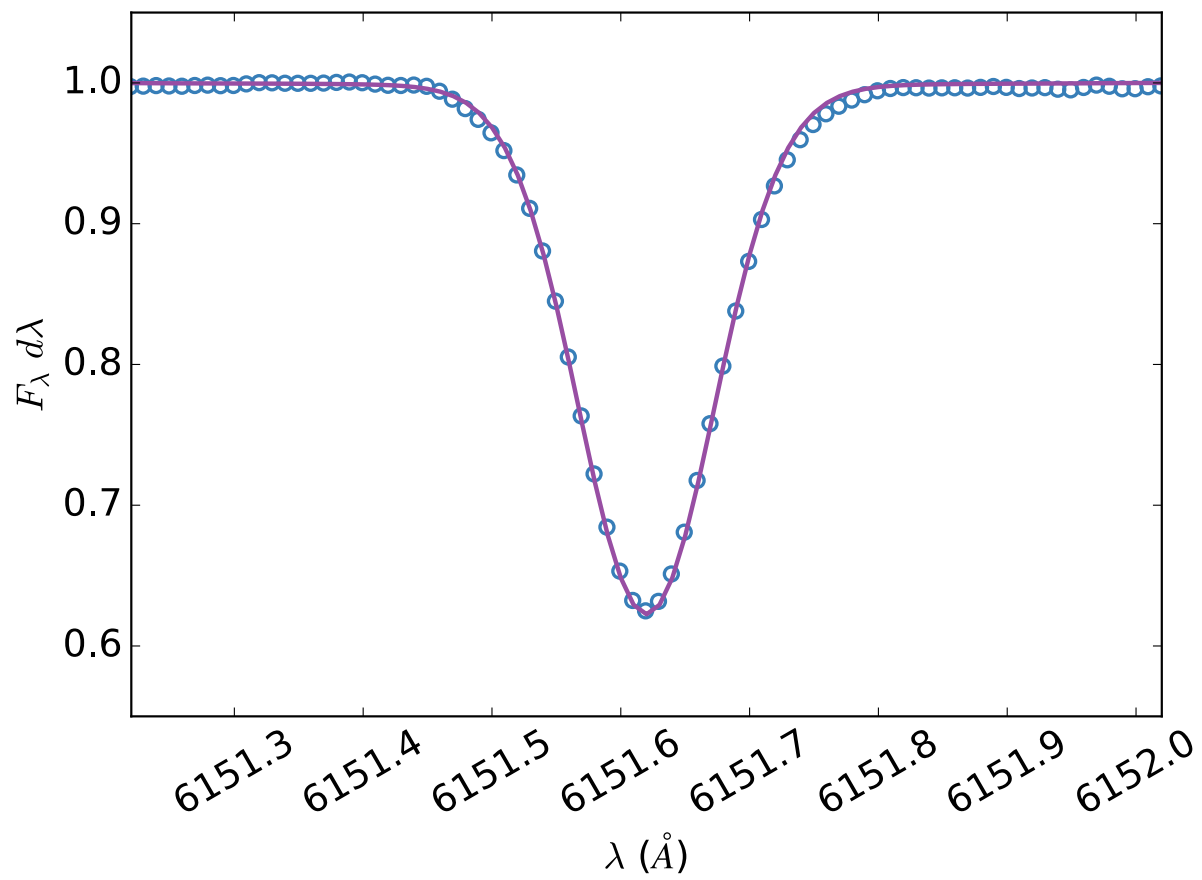

Figure 3.2: Example of line profile fitting for the Fe I feature at $6151.62 \AA$ in the spectrum of the Sun. The continuous curve is the synthetic spectrum, and the open circles are the observed data.

disentangle these two spectral line broadening processes; this is probably because of the low values of these velocities. Macroturbulence has a stronger effect on the wings of the spectral lines, but the selected clean lines still have some contamination that requires this high-precision work to be carried out by eye. Some stars show more contamination than others, complicating the disentanglement. Fortunately, the variation of macroturbulence with effective temperature and luminosity is smooth (Gray, 2005), so that precise values of $v_{\text {macro }}$ could be obtained by a calibration. Thus we adopted a relation that fixes macroturbulence velocities to measure $v \sin i$ with high precision using an automatic code, which provides the additional benefits of reproducibility and lower subjectivity.

The macroturbulence velocity is known to vary for different spectral lines (Gray, 2005), so for this high-precision analysis we do not adopt a single value for each star. Instead, we measure the $v_{\text {macro }}$ for the Sun in each of the spectral lines from Table 3.1 and use these values to scale the $v_{\text {macro }}$ for all stars in the sample using the following equation: 


$$
\begin{array}{r}
v_{\text {macro }, \lambda}^{*}=v_{\text {macro }, \lambda}^{\odot}-0.00707 T_{\text {eff }}+9.2422 \times 10^{-7} T_{\text {eff }}^{2} \\
+10.0+k_{1}(\log g-4.44)+k_{2} \\
\equiv f\left(T_{\text {eff }}\right)+k_{1}(\log g-4.44)+k_{2}
\end{array}
$$

where $v_{\text {macro, },}^{\odot}$ is the macroturbulence velocity of the Sun for a given spectral line, $T_{\text {eff }}$ and $\log g$ are the effective temperature and gravity of a given star, respectively, $k_{1}$ is a proportionality factor for $\log g$ and $k_{2}$ is a small correction constant.

This formula is partly based on the relation derived by Meléndez et al. (2012, Eq. E.1 in their paper) from the trend of macroturbulence with effective temperature in solar-type stars described by Gray (2005). The $\log g$-dependent term (a proxy for luminosity) comes from the empirical relation derived by Doyle et al. (2014, Eq. 8 in their paper), and is based on spectroscopic measurements of $v_{\text {macro }}$ of Kepler stars, which were disentangled from $v \sin i$ using asteroseismic estimates of the projected rotational velocities. Doyle et al. obtained a value for the proportionality factor $k_{1}$ of -2.0 . Their uncertainties on $v_{\text {macro }}$, however, were on the order of $1.0 \mathrm{~km} \mathrm{~s}^{-1}$. Thus, we decided to derive new values of $k_{1}$ and $k_{2}$ by simultaneously measuring $v_{\text {macro }}$ and $v \sin i$ of a subsample of solar twins.

This subsample was chosen to contain only single stars or visual binaries mostly in the extremes of $\log g(4.25-4.52)$ in the entire sample. We assume these values have a linear relationship with $v_{\text {macro }}$ inside this short interval of $\log g$. We used as a first guess the values of $v \sin i$ and $v_{\text {macro }}$ from a previous, cruder estimation we made, and performed line profile fits heuristically using MOOG synth. The velocities in Table 3.2 are the median of the values measured for each line and their standard error. These $v \sin i$ are not consistently measured in the same way as the final results. The rotational velocity broadening was calculated by our algorithm (see Sect. 3.4.2 for details). By performing a linear fit in the $v_{\text {macro }}-f\left(T_{\text {eff }}\right)$ versus $\log g-4.44$ relation $(f$ comprises all the $T_{\text {eff }}$-dependent terms, the macroturbulence velocity of the Sun and the known constant on Eq. 3.1), we obtain that $k_{1}=-1.81 \pm 0.26$ and $k_{2}=-0.05 \pm 0.03$ (see Fig. 3.3). For the stars farthest from the Sun in $\log g$ in the sample, these values of $k_{1}$ and $k_{2}$ would amount to differences of up to $\pm 0.4 \mathrm{~km} \mathrm{~s}^{-1}$ in their macroturbulence velocities, therefore it is essential to consider the luminosity effect on $v_{\text {macro }}$ for accurate $v \sin i$ determinations.

To obtain the macroturbulence velocities for the Sun to use in Eq. 3.1, we forced the rotational velocity of the Sun to $1.9 \mathrm{~km} \mathrm{~s}^{-1}$ (Howard and Harvey, 1970), and then estimated values of 
Table 3.2 - Simultaneous measurements of rotational and macroturbulence velocities of stars in the extremes of $\log g$ from the sample of solar twins.

\begin{tabular}{lcccc}
\hline Star & $\begin{array}{c}v \sin i \\
\left(\mathrm{~km} \mathrm{~s}^{-1}\right)\end{array}$ & $\begin{array}{c}v_{\text {macro }} \\
\left(\mathrm{km} \mathrm{s}^{-1}\right)\end{array}$ & $T_{\text {eff }}$ & $\log g$ \\
\hline HIP 115577 & $0.95 \pm 0.05$ & $3.35 \pm 0.09$ & 5699 & 4.25 \\
HIP 65708 & $1.20 \pm 0.09$ & $3.55 \pm 0.08$ & 5755 & 4.25 \\
HIP 74432 & $1.40 \pm 0.03$ & $3.35 \pm 0.08$ & 5684 & 4.25 \\
HIP 118115 & $1.40 \pm 0.10$ & $3.43 \pm 0.09$ & 5808 & 4.28 \\
HIP 68468 & $1.75 \pm 0.07$ & $3.70 \pm 0.08$ & 5857 & 4.32 \\
HIP 41317 & $1.55 \pm 0.03$ & $3.10 \pm 0.06$ & 5700 & 4.38 \\
Sun & $1.75 \pm 0.07$ & $3.30 \pm 0.06$ & 5777 & 4.44 \\
HIP 105184 & $2.50 \pm 0.03$ & $3.21 \pm 0.08$ & 5833 & 4.50 \\
HIP 10175 & $1.55 \pm 0.06$ & $3.05 \pm 0.08$ & 5738 & 4.51 \\
HIP 114615 & $2.20 \pm 0.03$ & $3.25 \pm 0.08$ & 5816 & 4.52 \\
HIP 3203 & $3.90 \pm 0.03$ & $3.40 \pm 0.10$ & 5850 & 4.52 \\
\hline
\end{tabular}

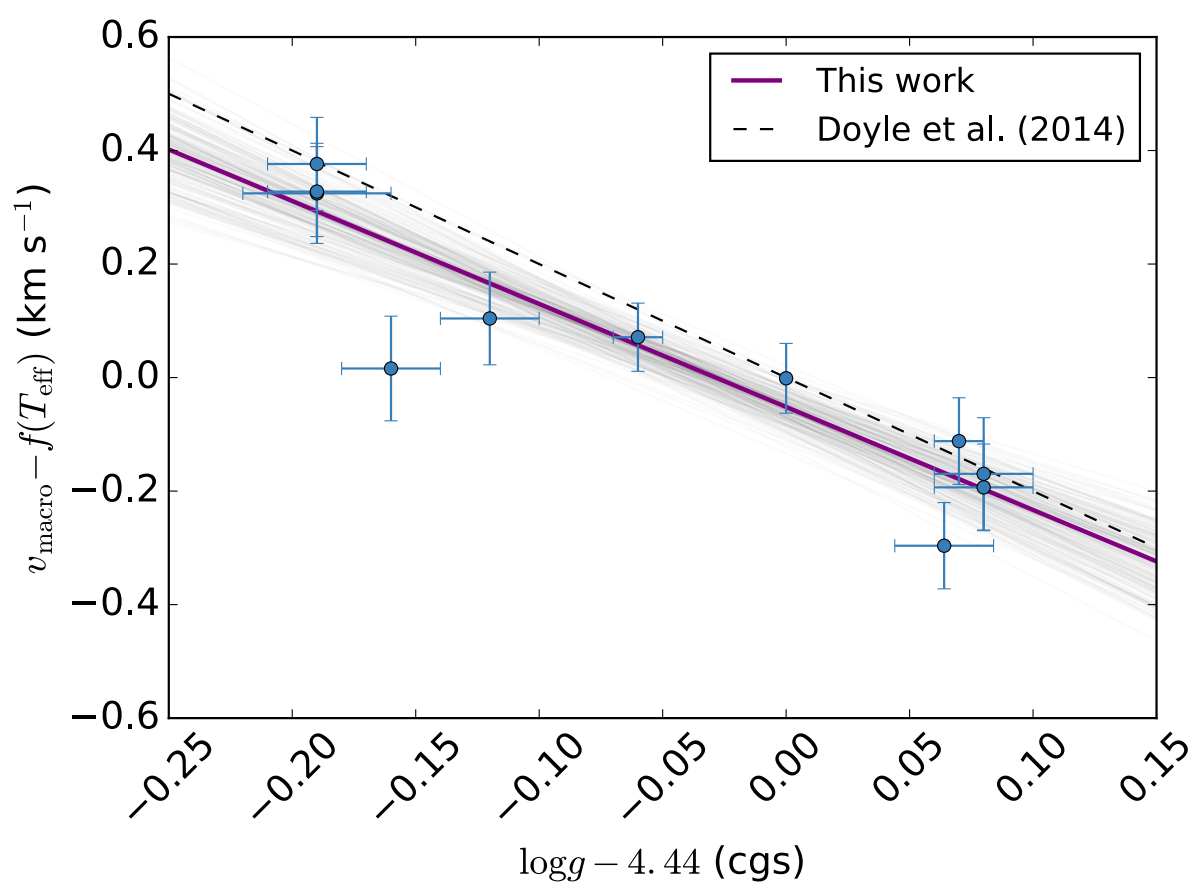

Figure 3.3: Linear relation between $v_{\text {macro }}$ and $\log g$ (a proxy for luminosity) for the stars on Table 3.2. See the definition of $f\left(T_{\text {eff }}\right)$ in Sect. 3.4.1. The purple continuous line represents our determination of a proportionality coefficient of -1.81 and a vertical shift of $-0.05 \mathrm{~km} \mathrm{~s}^{-1}$. The black dashed line is the coefficient found by Doyle et al. (2014). The light gray region is a composition of 200 curves with parameters drawn from a multivariate Gaussian distribution. The Sun is located at the origin. 
$v_{\text {macro, },}^{\odot}$ by fitting each line profile using MOOG synth, and the results are shown in Table 3.1. We estimated the error in determining $v_{\text {macro, },}^{\odot}$ to be $\pm 0.1 \mathrm{~km} \mathrm{~s}^{-1}$. Since Eq. 3.1 is an additive scaling, the error for $v_{\text {macro }}$ of all stars is the same as in the Sun. The uncertainties in stellar parameters have contributions that are negligible compared to the ones introduced by the error in $v_{\text {macro }}$.

\subsubsection{Rotational velocities}

Our code takes as input the list of stars and their parameters (effective temperature, surface gravity, metallicity and microturbulence velocities obtained in Paper I), their spectra and the spectral line list in MOOG-readable format. For each line in a given star, the code automatically corrects the spectral line shift and the continuum. The first is performed by fitting a secondorder polynomial to the kernel of a line and estimating the distance of the observed line center from the laboratory value. Usually, the spectral line shift corrections were on the order of $10^{-2}$ $\AA$, corresponding to $0.5 \mathrm{~km} \mathrm{~s}^{-1}$ in the wavelength range we worked on. This is a reasonable shift that likely arises from a combination of granulation and gravitational redshift effects, which are of similar magnitude. The continuum correction for each line is defined as the value of a multiplicative factor that sets the highest flux inside a radius of $2.5 \AA$ around the line center to 1.0. The multiplicative factor usually has a value inside the range $1.000 \pm 0.002$.

The code starts with a range of $v \sin i$ and abundances and optimizes these two parameters through a series of iterations that measure the least-squares difference between the observed line and the synthetic line (generated with MOOG synth). Convergence is achieved when the difference between the best and previous solutions, for both $v \sin i$ and abundance, is less than $1 \%$. Additionally, the code also forces at least ten iterations to avoid falling into local minima.

One of the main limitations of MOOG synth for our analysis is that it has a "quantized" behavior for $v \sin i$ : the changes in the synthetic spectra occur most strongly in steps of $0.5 \mathrm{~km}$ $\mathrm{s}^{-1}$. This behavior is not observed in varying the macroturbulence velocities. Therefore, we had to incorporate a rotational broadening routine in our code that was separated from MOOG. We used the Eq. 1.15 to compute the rotational profile ${ }^{5}$. The rotational profile $G(\mathrm{v})$ is then convolved with the MOOG synthetic profiles, which were generated with $v \sin i=0$.

The total uncertainties in rotational velocities are obtained from the quadratic sum of the standard error of the five measurements and an uncertainty of $0.1 \mathrm{~km} \mathrm{~s}^{-1}$ introduced by the error in macroturbulence velocities. Systematic errors in the calculation of $v_{\text {macro }, \lambda}$ for the stars do not

\footnotetext{
${ }^{5}$ This is the same recipe adopted by the radiative transfer code MOOG.
} 
significantly contribute to the $v \sin i$ uncertainties. The rotational velocities we measured and their uncertainties are reproduced in Appendix A.

Some of the stars in the sample show very low rotational velocities, most probably owing to the effect of projection (see Fig. 3.5). The achieved precision is validated by comparison with the values of the full width at half maximum (FWHM) measured by the cross-correlation function (CCF) from the data reduction pipeline, with the effects of macroturbulence subtracted (see Fig. 3.4). The spectroscopic binary star HIP 103983 has an unusually high $v \sin i$ when compared to the CCF FWHM, and a verification of its spectral line profiles reveals the presence of distortions, which are the most probably caused by mismeasurement of rotational velocity (contamination of the combined spectrum by a companion; observations range from October 2011 to August 2012). We obtained a curve fit for the $v \sin i$ versus CFF FWHM $\left(\mathrm{km} \mathrm{s}^{-1}\right)$ using a similar relation as used by Melo et al. (2001); Pace and Pasquini (2004); Hekker and Meléndez (2007), which resulted in the following calibration:

$$
v \sin i=\sqrt{(0.73 \pm 0.02)\left[\mathrm{FWHM}^{2}-v_{\text {macro }}^{2}-(5.97 \pm 0.01)^{2}\right]} \mathrm{km} \mathrm{s}^{-1}
$$

(estimation performed with the MCMC code emcee, ${ }^{6}$ Foreman-Mackey et al., 2013). The scatter between the measured $v \sin i$ and those estimated from CCF is $\sigma=0.20 \mathrm{~km} \mathrm{~s}^{-1}$ (excluding the outlier HIP 103983). The typical uncertainty in the rotational velocities we obtain with our method, that is, line profile fitting with extreme high-resolution spectra, is $0.12 \mathrm{~km} \mathrm{~s}^{-1}$, which implies that the average error of the CCF FWHM $v \sin i$ scaling is $0.16 \mathrm{~km} \mathrm{~s}^{-1}$. This average error could be significantly higher if the broadening by $v_{\text {macro }}$ is not accounted for.

\subsection{Binary stars}

We identified 18 spectroscopic binaries (SB) in the sample of 81 solar twins by analyzing their radial velocities; some of these stars are reported as binaries by Tokovinin (2014a,b); Mason et al. (2001); Baron et al. (2015). We did not find previous reports of multiplicity for the stars HIP 6407, HIP 30037, HIP 54582 and HIP 62039 in the literature; the binaries HIP 19911 and HIP 103983 are confirmed to be double-lined (see Chapter 4). The star HIP 64150 is a Siriuslike system with a directly observed white dwarf companion (Crepp et al., 2013; Matthews et al., 2014). The sample from Paper I contains another SB, HIP 109110, for which we could not

\footnotetext{
${ }^{6}$ Available at http://dan.iel.fm/emcee/current/
} 


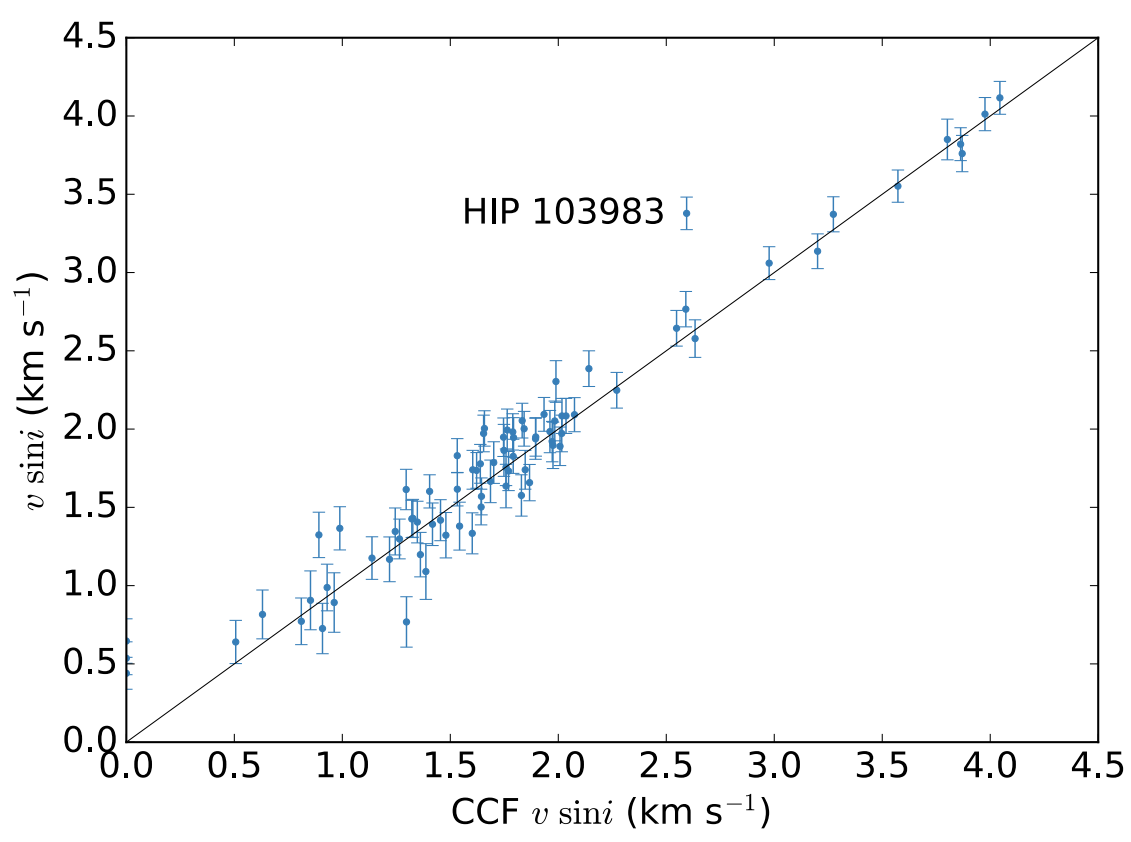

Figure 3.4: Comparison between the estimated values of $v \sin i$ (y-axis) and those inferred from the cross-correlation funcion FWHM (x-axis). The spread around the 1:1 relation (black line) is $\sigma=0.20 \mathrm{~km} \mathrm{~s}^{-1}$.

reliably determine the $v \sin i$ because of strong contamination in the spectra, which is possibly caused by a relatively bright companion. Thus, we did not include this star in the sample.

Of these 18 spectroscopic binaries, at least two of them (HIP 19911 and 67620) show unusually high $v \sin i$ (see Fig. 3.5). These stars also display other abnormalities, such as their [Y/Mg] abundances (Tucci Maia et al., 2016) and magnetic activity (Ramírez et al., 2014; Freitas et al., 2017). The solar twin blue straggler HIP 10725 (Schirbel et al., 2015), which is not included in the sample, also shows a high $v \sin i$ for its age. We find that eight of the binaries have rotational velocities below the expected for Sun-like stars, but this is most likely an effect of projection of the rotational axes of the stars. For the remaining binaries, which follow the rotational braking law, it is again difficult to disentangle this behavior from the $\sin i$, and a statistical analysis is precluded by the low numbers involved. Tidal interactions between companions that could potentially enhance rotation depend on binary separation, but these anomalies could also be explained by contamination by bright companions; these hypotheses are discussed in Chapter 4 . They should be regular rotators, since they do not show abnormalities in chromospheric activity (Freitas et al., 2017) or [Y/Mg] abundances (Tucci Maia et al., 2016). 


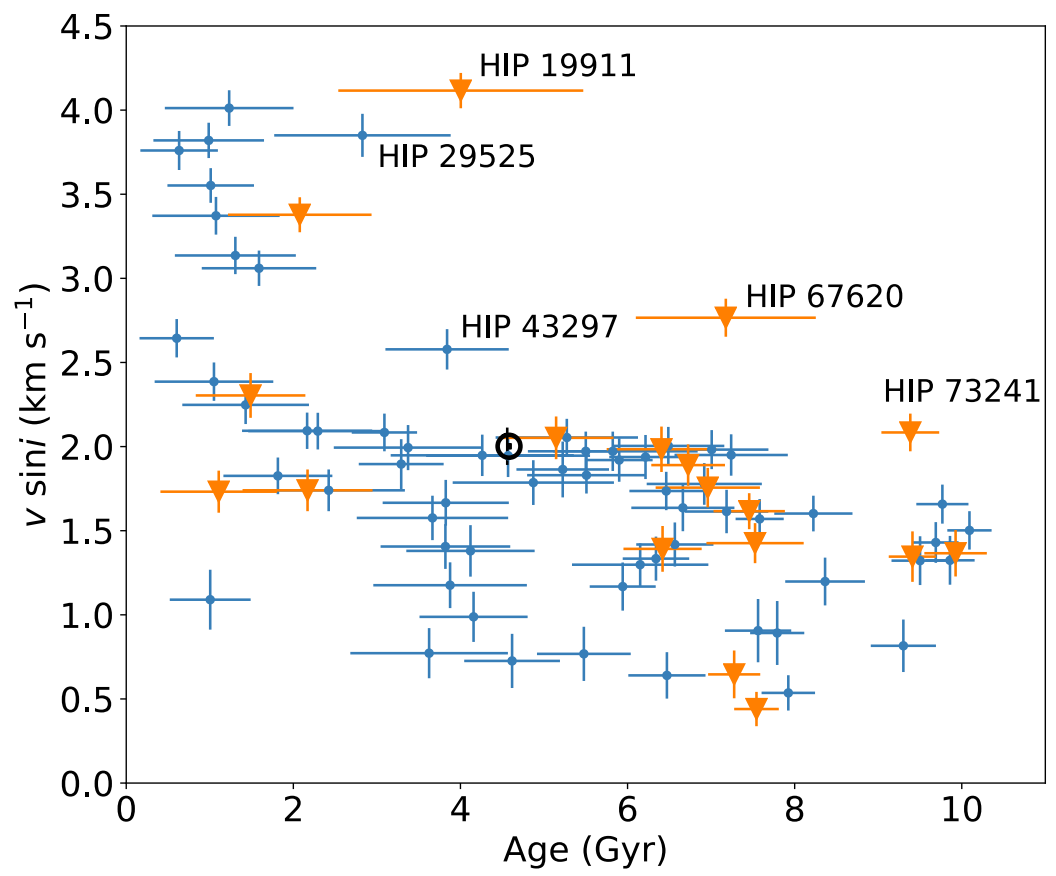

Figure 3.5: Projected rotational velocity of solar twins as a function of their age. The Sun is represented by the symbol $\odot$. The orange triangles are spectroscopic binaries, and the blue dots are the nonspectroscopic binaries.

\subsection{The rotational braking law}

We removed from this analysis all the spectroscopic binaries in order to correctly constrain the rotational braking. The non-SB HIP 29525 and HIP 43297 display $v \sin i$ that are much higher than expected, but preliminary age estimates using the HARPS spectra (Spina et al., in preparation) point out that they are likely to have overestimated isochronal ages; thus we decided to not include HIP 29525 and HIP 43297 in the rotational braking determination. Maldonado et al. (2010) found X-ray and chromospheric ages of 0.55 and 0.17 Gyr, respectively, for HIP 29525. We then divided the remaining 61 stars and the Sun in bins of $2 \mathrm{Gyr}$, and removed all the stars that were below the 70th percentile of $v \sin i$ in each bin from this sample. Such a procedure can be justified because we verified that $30 \%$ of the stars should have $\sin i$ above 0.9 by doing a simple simulation with angles $i$ drawn from a flat distribution between 0 and $\pi / 2$. This allowed us to select the stars that had the highest chance of having $\sin i$ above 0.9. In total, 21 solar twins and the Sun compose what we hereafter reference as the selected sample. Albeit this subsample is smaller, it has the advantage of mostly removing uncertainties on the inclination angle of the 


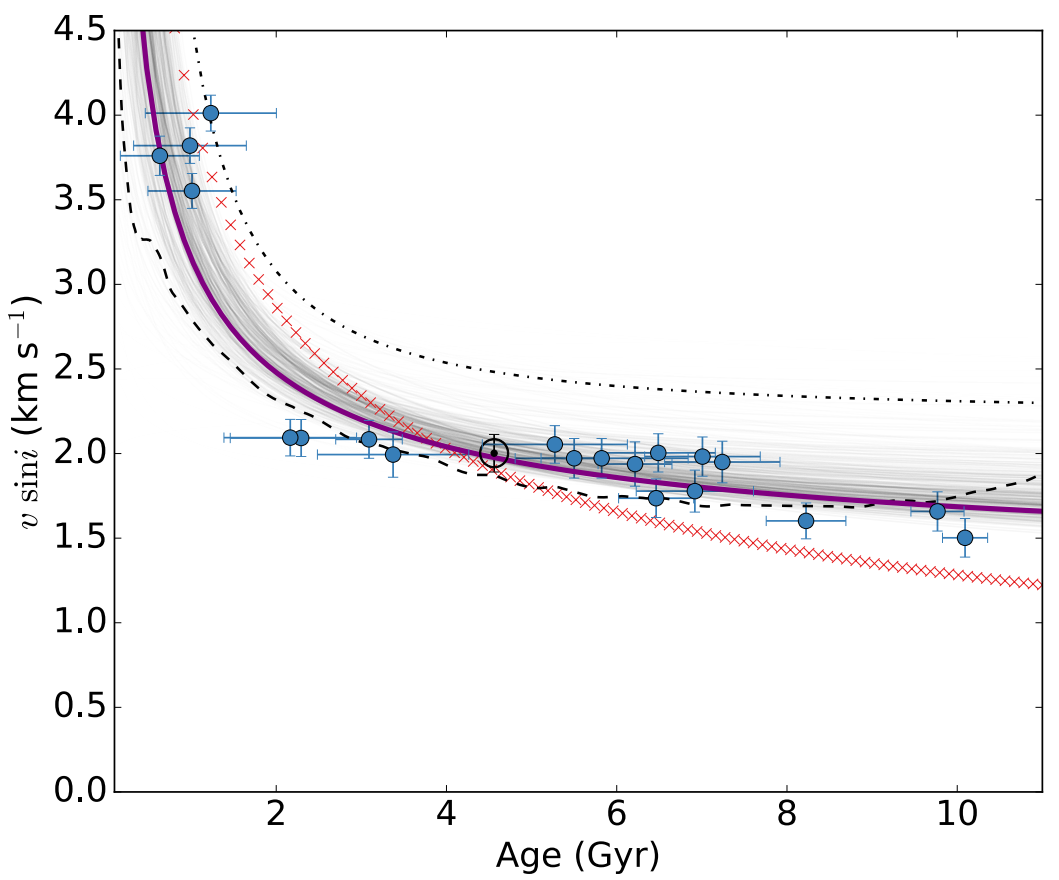

Figure 3.6: Rotational braking law of the stars in our program. The purple continuous curve is the relation inferred from fitting the selected sample (blue circles) of solar twins with the form $v \sin i=v_{\mathrm{f}}+m t^{-b}$, where $t$ is the stellar age, and the fit parameters are $v_{\mathrm{f}}=1.224 \pm 0.447, m=1.932 \pm 0.431$, and $b=0.622 \pm 0.354$, with $v_{\mathrm{f}}$ and $b$ highly and positively correlated. The light gray region is composed of 300 curves that are created with parameters drawn from a multivariate Gaussian distribution defined by the mean values of the fit parameters and their covariance matrix. Skumanich's law (red $\times$ symbols, calibrated for $v_{\text {rot }}^{\odot}=1.9 \mathrm{~km} \mathrm{~s}^{-1}$ ) and the rotational braking curves proposed by do Nascimento et al. (2014, black dashed curve, smoothed) and Pace and Pasquini (2004, black dot-dashed curve) are plotted for comparison.

stellar rotation axes ${ }^{7}$. We stress that the only reason we can select the most probable edge-on rotating stars $(i=\pi / 2)$ is because we have a large sample of solar twins in the first place.

We then proceeded to fit a general curve to the selected sample (see Fig. 3.6) using the method of orthogonal distance regression (ODR, Boggs and Rogers, 1990), which takes the uncertainties on both $v \sin i$ and ages into account. This curve is a power law plus constant of the form $v=v_{\mathrm{f}}+m t^{-b}$ (the same chromospheric activity and $v \sin i$ vs. age relation used by Pace and Pasquini, 2004; Guinan and Engle, 2009), with $v$ (rotational velocity) and $v_{\mathrm{f}}$ (asymptotic velocity) in $\mathrm{km} \mathrm{s}^{-1}$ and $t$ (age) in Gyr.

We find that the best-fit parameters are $v_{\mathrm{f}}=1.224 \pm 0.447, m=1.932 \pm 0.431$, and $b=$ $0.622 \pm 0.354$ (see Fig. 3.6). These large uncertainties are likely due to i) the strong correlation between $v_{\mathrm{f}}$ and $b$; and ii) the relatively limited number of data points between 1 and $4 \mathrm{Gyr}$,

\footnotetext{
${ }^{7}$ This procedure can also allow for unusually fast-rotating stars (although rare) with sin $i$ below 0.9 to leak into the subsample.
} 
where the parameter is most effective in changing the values of $v$. This limitation is also present in past studies (e.g., van Saders et al., 2016; Barnes, 2003; Pace and Pasquini, 2004; Mamajek and Hillenbrand, 2008; García et al., 2014; Amard et al., 2016). On the other hand, the sample is the largest comprising solar twins and, therefore, should produce more reliable results. With more data points, we could use $1 \mathrm{Gyr}$ bins instead of $2 \mathrm{Gyr}$ in order to select the fastest rotating stars, which would result in a better subsample for constraining the rotational evolution for young stars.

The relation we obtain is in contrast with some previous studies on modeling the rotational braking (Barnes, 2001, 2003; Lanzafame and Spada, 2015) which either found or assumed that the Skumanich law explains well the rotational braking of Sun-like stars. The conclusions by van Saders et al. (2016) limit the range of validation up to approximately the solar age (4 Gyr) for stars with solar mass. When we enforce the Skumanich power-law index $b=1 / 2$, we obtain a worse fit between the ages 2 and 4 Gyr and, not surprisingly, after the solar age as well.

The HARPS data and the rotational braking law that results from them show that the Sun is a normal star regarding its rotational velocity when compared to solar twins. However, they do not agree with the regular Skumanich law (Barnes, 2007, red $\times$ symbols in Fig. 3.6). We find a better agreement with the model proposed by do Nascimento et al. (2014, black dashed curve in Fig. 3.6), especially for stars older than 2 Gyr. This model is thoroughly described in Appendix A of do Nascimento et al. (2012). In summary, this model uses an updated treatment of the instabilities that are relevant to the transport of angular momentum, according to Zahn (1992) and Talon and Zahn (1997), with an initial angular momentum for the Sun $J_{0}=1.63 \times 10^{50} \mathrm{~g} \mathrm{~cm}^{2}$ $\mathrm{s}^{-1}$. The rotational braking curve that corresponds to the model by do Nascimento et al. (2014) is computed using the output radii of the model, which vary from $\sim 1 \mathrm{R}_{\odot}$ at the current solar age to $1.57 \mathrm{R}_{\odot}$ at the age of $11 \mathrm{Gyr}$, and it changes significantly if we use a constant radius $R=1$ $\mathrm{R}_{\odot}$. This results in a more Skumanich-like rotational braking.

Our result agrees with the chromospheric activity versus age behavior for solar twins obtained by Ramírez et al. (2014), in which a steep decay of the $R_{\mathrm{HK}}^{\prime}$ index during the first 4 Gyr was deduced (see Fig. 11 in their paper). The study by Pace and Pasquini (2004) also suggests a steeper power-law index $(b=1.47)$ than Skumanich's $\left(b_{\mathrm{S}}=1 / 2\right)$ in the rotational braking law derived from young open clusters, the Sun and M 67. As seen in Fig. 3.6, however, their relation significantly overestimates the rotational velocities of stars, especially for those older than 2 Gyr. This is most probably caused by other line broadening processes, mainly the macroturbulence, 
which were not considered in that study. As we saw in Sect. 3.4.1, those processes introduce important effects that are sometimes larger than the rotational broadening. Moreover, a CCF-only analysis tends to produce more spread in the $v \sin i$ than the more detailed analysis we used.

The rotational braking law we obtain produces a similar outcome to that achieved by van Saders et al. (2016) for stars older than the Sun, that is, a weaker rotational braking law after solar age than previously suggested. Our data also requires a different power-law index than the Skumanich index for stars younger than the Sun, accounting for an earlier decay of rotational velocities up to 2 Gyr.

Based on the main-sequence spin-down model by Kawaler (1988, see Section 1.2), if we assume a dipole geometry for the stellar magnetic field $\left(B_{\mathrm{r}} \propto B_{0} r^{3}\right)$, then the value of the free parameter $n$ in equation 1.7 is 3/7. Furthermore, assuming that $a=1$, then Eq. 1.7 results in $v_{\mathrm{eq}} \propto t^{-7 / 4}=t^{-1.75}$. The Skumanich law $\left(v_{\mathrm{eq}} \propto t^{-0.5}\right)$ is recovered for $n=3 / 2$, which is close to the case of a purely radial field $\left(n=2, v_{\mathrm{eq}} \propto t^{-0.38}\right)$. A more extensive exploration of the configuration and evolution of magnetic fields of solar twins is outside the scope of this paper, but our results suggest that the rotational rotational braking we observe on this sample of solar twins stems from a magnetic field with an intermediate geometry between dipole and purely radial.

\subsection{Conclusions of this chapter}

We analyzed the rotational velocities of 81 bright solar twins in the Southern Hemisphere and the Sun using extremely high-resolution spectra. Radial velocities revealed that the sample contained 18 spectroscopic binaries, four of which (HIP 6407, 30037, 54582, 62039) were not listed as such in the literature. Two of these stars show an enhancement on their measured $v \sin i$, and they also present other anomalies in chemical abundances and chromospheric activities. We also found two nonspectroscopic binary stars with unusually high rotational velocities for their age, but the revised HARPS ages of these systems (Spina et al., in preparation) point out that they are young stars and thus not outliers.

We selected a subsample of stars with higher chances of having their rotational axis inclination close to $\pi / 2$ (almost edge-on) in order to better constrain the rotational evolution of the solar twins. We opted to use carefully-measured isochronal ages for these stars because it is the most reliable method available for this sample. We finally conclude that the Sun seems to be a 
common rotator, within the uncertainties, when compared to solar twins, therefore it can be used to calibrate stellar models.

Moreover, we have found that the Skumanich law does not describe well the rotation evolution for solar twins observed in the data, which is a discrepancy that is stronger after the solar age. Therefore, we propose a new rotational braking law that supports the weakened braking after the age of the Sun, and comes with a earlier decay in rotational velocities up to 2 Gyr than the classical Skumanich's law. Interestingly, it also reveals an evolution that is more similar to the magnetic activity evolution observed in Sun-like stars, which sees a steep decay in the first $3 \mathrm{Gyr}$ and flattens near the solar age. Additionally, we suggest that more high-precision spectroscopic observations of solar twins younger and much older than the Sun could help us better constrain the rotational evolution of solar-like stars. 
Chapter 4

\section{New and improved orbital constraints of solar twin binaries}

This chapter is based on the article accepted for publication dos Santos et al. (2017). The coauthors J. Meléndez, M. Bedell, J. L. Bean, L. Spina, A. Alves-Brito, S. Dreizler, I. Ramírez and M. Asplund contributed with the observations, data reduction, data analysis and discussion of the results. A copy of the accepted article can be found in Appendix B.

\subsection{Abstract of this chapter}

Previous studies on the rotation of Sun-like stars revealed that the rotational rates of young stars converge towards a well-defined evolution that follows a power-law decay. It seems, however, that some binary stars do not obey this relation, often by displaying enhanced rotational rates and activity. In the Solar Twin Planet Search program, we observed several solar twin binaries, and found a multiplicity fraction of $42 \% \pm 6 \%$ in the whole sample; moreover, at least three of these binaries (HIP 19911, HIP 67620 and HIP 103983) clearly exhibit the aforementioned anomalies. We investigated the configuration of the binaries in the program, and discovered new companions for HIP 6407, HIP 54582, HIP 62039 and HIP 30037, of which the latter is orbited by a $0.06 \mathrm{M}_{\odot}$ brown dwarf in a 1-month long orbit. We report the orbital parameters of the systems with well-sampled orbits and, in addition, the lower limits of parameters for the companions that only display a curvature in their radial velocities. For the linear trend binaries, we report an estimate of the masses of their companions when their observed separation is available, and a minimum mass otherwise. We conclude that solar twin binaries with low-mass stellar companions at moderate orbital periods do not display signs of a distinct rotational evolution when compared to single stars. We confirm that the three peculiar stars are double-lined binaries, and 
that their companions are polluting their spectra, which explains the observed anomalies.

\subsection{Do binary solar twins follow a distinct evolutionary path?}

It is known that at least half of the stars in the Galaxy are multiple systems containing two or more stars orbiting each other (Kiseleva-Eggleton and Eggleton, 2001; Fuhrmann et al., 2017), thus in many surveys and large samples of stars, binaries are ubiquitous. This is in contrast with the Sun, which is a single star, and attempts to find a faint stellar companion orbiting it rendered no results thus far (e.g., Luhman, 2014). Many studies avoid contamination by binaries in their samples, the main reasons being because we do not understand well how binaries evolve and how the presence of a companion affects the primary star. However, with the development of instruments with higher spatial and spectral resolution and coronagraphs, it is now possible to better probe the secondary component of such systems.

We have been carrying out a radial velocity planet search focused on solar twins using HARPS (Ramírez et al., 2014; Bedell et al., 2015; Tucci Maia et al., 2016; dos Santos et al., 2016; Meléndez et al., 2017, hereafter Papers I, II, III, IV and V, respectively). The definition of solar twin we use is a star with stellar parameters inside the ranges $5777 \pm 100 \mathrm{~K}, 4.44 \pm 0.10$ $\operatorname{dex}(\mathrm{cgs})$ and $0.0 \pm 0.1 \mathrm{dex}$, respectively, for $T_{\text {eff }}, \log g$ and $[\mathrm{Fe} / \mathrm{H}]$. In total, 81 solar twins $^{1}$ were observed on HARPS. As part of our survey we previously identified 16 clear spectroscopic binaries (SB) (Paper IV). We report here the identification of four additional SBs (HIP 14501, HIP 18844, HIP 65708 and HIP 83276) and the withdrawal of HIP 43297 and HIP 64673, which are unlikely to host stellar-mass companions, bringing the number of solar twin SBs to 18. Most of these SBs are single-lined - they do not contain a second component in their spectral lines -, meaning that their companions are either faint stars or located outside the $\sim 1$ " aperture of the HARPS spectrograph. We confirm that there are three solar twins with spectra contaminated by a relatively bright companion (see discussion in Section 4.5.3). In our sample there are an additional 18 visual binaries $^{2}$ or multiple systems, of which HIP 6407 and HIP 18844 have wide companions (see table 5 in Tokovinin, 2014a) as well as the spectroscopic companions reported here.

In Paper IV we saw that the single or visual binary solar twins display a rotational evolution

\footnotetext{
${ }^{1}$ Some of the stars in our sample do not fit the strict definition of solar twins because one or more parameters are off the definition intervals, but they are still very close solar analogues.

${ }^{2}$ We define as visual companions those with separations larger than $1^{\prime \prime}$
} 
that can described with a relation between stellar age $t$ and rotational velocity $v_{\text {rot }}$ in the form of a power law plus a constant: $v_{\text {rot }}=v_{\mathrm{f}}+m t^{-b}$, where $v_{\mathrm{f}}, m$ and $b$ are free parameters to be fit with observations. This relation is explained by loss of angular momentum due to magnetized winds (e.g., Mestel, 1984; Charbonneau, 1992; Barnes, 2003; Gallet and Bouvier, 2013), and the index $b$ reflects the geometry of the stellar magnetic field (Kawaler, 1988). There are at least two solar twin binaries that display enhanced rotational velocities - above $2 \sigma$ from the expected - and activity for their ages: HIP 19911 and HIP 67620; if we consider the revised age for HIP 103983 (Spina et al., in preparation), it can also be considered a fast rotator for its age.

Besides the enhanced rotational velocities and higher chromospheric activity (Paper I; Freitas et al. 2017), some of these binaries also display peculiar chemical abundances (Paper III; Desidera et al. 2016). As pointed out by Desidera et al., the ultra-depletion of beryllium, which is observed on HIP 64150, could be explained by the interaction of the main star with the progenitor of the white dwarf companion. In addition to HIP 64150, the confirmed binaries HIP 19911 and HIP 67620 also display clearly enhanced [Y/Mg] abundances (see fig. 9 in Paper III, and the discussion in Section 4.5.3 of this paper).

One interesting aspect about stars with enhanced activity and rotation is that these characteristics were hypothesized to be the result of dynamo action from close-in giant planets (see Karoff et al., 2016, and references therein). In fact, some of our early results pointed out that the star HIP 68468, for which we inferred two exoplanets candidates (Paper V), had an enhanced rotational velocity when compared to other solar twins of the same age. However, a more careful analysis showed that the enhancement was instead a contribution of macroturbulence. Another explanation for these enhancements is due to magnetic interactions with either a close-in or an eccentric giant planet (Cuntz et al., 2000), but recent results obtained by, e.g., Figueira et al. (2016) and Mengel et al. (2017) show that they cannot explain such anomalies.

In light of these intriguing results, we sought to better understand the nature of these solar twin multiple systems by studying their orbital parameters, and use them to search for explanations of the observed anomalies, especially stellar rotation. The orbital parameters can be estimated from the radial velocity data of the stars (see, e.g., Murray and Correia, 2010, hereafter $\mathrm{MC10}$ ), with the quality of the results depending strongly on the time coverage of the data. 


\subsection{Radial velocities}

Our solar twins HARPS data $^{3}$ are completely described in Paper I. Their radial velocities (RV) are automatically measured from the HARPS Data Reduction Software, and the noise limit of the instrument generally remains around $1 \mathrm{~m} \mathrm{~s}^{-1}$. In order to broaden the coverage of our RV data, we also obtained more datasets that were available in the literature and public databases, including the HARPS archival data for other programs.

The mass and other stellar parameters of the solar twins were estimated with high precision using the combined HARPS spectra and differential analysis owing to their similarity with the Sun (see, e.g., Bedell et al., 2014; Baumann et al., 2010; Yana Galarza et al., 2016). The ages for the solar twins were obtained using Yonsei-Yale isochrones (Yi et al., 2001) and probability distribution functions as described in Ramírez et al. (2013) and in Paper I. The full description and discussion of the stellar parameters of the HARPS sample are going to be presented in a forthcoming publication (Spina et al., in preparation).

The additional radial velocities data obtained from online databases and the literature are summarized in Table 4.1. These are necessary to increase the time span of the observations to include as many orbital phases as possible at the cost of additional parameters to optimize for (see Section 4.4.1).

Table 4.1 - Additional radial velocities from other programs and instruments.

\begin{tabular}{lll}
\hline Instrument/Program & References & Data available for (HIP numbers) \\
\hline CfA Digital Speedometers & Latham et al. (2002) & 65708 \\
ELODIE & Moultaka et al. (2004) & $43297,54582,62039,64150,72043,87769$ \\
SOPHIE & Perruchot et al. (2011) & $6407,43297,54582,62039,64150,87769$ \\
Lick Planet Search & Fischer et al. (2014) & 54582,65708 \\
AAT Planet Search & Jenkins et al. (2015) & $18844,67620,73241,79578,81746$ \\
Various & Willmarth et al. (2016) & 67620 \\
HIRES/Keck RV Survey & Butler et al. (2017) & $14501,19911,62039,64150,72043,103983$ \\
\hline
\end{tabular}

\footnotetext{
${ }^{3}$ Based on observations collected at the European Organisation for Astronomical Research in the Southern Hemisphere under ESO programs 188.C-0265, 183.D-0729, 292.C-5004, 077.C-0364, 072.C-0488, 092.C-0721, 093.C-0409, 183.C-0972, 192.C-0852, 091.C-0936, 089.C-0732, 091.C-0034, 076.C-0155, 185.D-0056, 074.C0364, 075.C-0332, 089.C-0415, 60.A-9036, 075.C-0202, 192.C-0224, 090.C-0421 and 088.C-0323.
} 


\subsection{Methods}

The variation of radial velocities of a star in binary or multiple system stems from the gravitational interaction between the observed star and its companions. For systems with stellar or substellar masses, the variation of radial velocities can be completely explained by the Keplerian laws of planetary motion. For the sake of consistency, we will use here the definitions of orbital parameters as presented in MC10.

To completely characterize the orbital motion of a binary system from the measured radial velocities of the main star, we need to obtain the following parameters: the semi-amplitude of the radial velocities $K$, the orbital period $T$, the time of periastron passage $t_{0}$, the argument of periapse $\omega$ and the eccentricity $e$ of the orbit. In order to estimate the minimum mass $m \sin i$ of the companion and the semi-major axis $a$ of the orbit, we need to know the mass $M$ of the main star.

Due to their non-negative nature, the parameters $K$ and $T$ are usually estimated in logarithmic scale in order to eliminate the use of search bounds. Additionally, for orbits that are approximately circular, the value of $\omega$ may become poorly defined. In these cases, a change of parametrization may be necessary to better constrain them. Eastman et al. (2013), for instance, suggest using $\sqrt{e} \cos \omega$ and $\sqrt{e} \sin \omega$ (which we refer to as the EXOFAST parametrization) instead of $\omega$ and $e$ to circumvent this problem, which also can help improve convergence time.

One issue that affects the radial velocities method is the contamination by stellar activity (see, e.g., Haywood et al., 2016). This activity distorts the spectral lines (Gray, 2005), which in turn produces artificial RV variations that can mimic the presence of a massive companion orbiting the star. More active stars are expected to have RV variations with larger amplitudes and a shorter activity cycle period (Lovis et al., 2011). For most binaries in our sample, the contamination by activity in the estimation of orbital parameters is negligible; the cases where this is not applicable are discussed in detail.

\subsubsection{Binaries with well-sampled orbits}

For binaries with orbital periods $T \lesssim 15 \mathrm{yr}$, usually there are enough RV data measured to observe a complete phase. In these cases, the natural logarithm of the likelihood of observing radial velocities $\mathbf{y}$ on a specific instrument, given the Julian dates $\mathbf{x}$ of the observations, their uncertainties $\sigma$ and the orbital parameters $\mathbf{p}_{\text {orb }}$ is defined as: 


$$
\ln p\left(\mathbf{y} \mid \mathbf{x}, \sigma, \mathbf{p}_{\text {orb }}\right)=-\frac{1}{2} \sum_{n}\left[\frac{\left(y_{\mathrm{n}}-y_{\text {model }}\right)^{2}}{\sigma_{\mathrm{n}}^{2}}+\ln \left(2 \pi \sigma_{\mathrm{n}}^{2}\right)\right],
$$

where $y_{\mathrm{n}}$ are the RV datapoints, $y_{\text {model }}$ are the model RV points for a given set of orbital parameters, and $\sigma_{\mathrm{n}}$ are the RV point-by-point uncertainties. The RV models are computed from Eq. 65 in MC10:

$$
v_{\mathrm{r}}=\gamma+K(\cos (\omega+f)+e \cos \omega),
$$

where $f$ is the true anomaly, and $\gamma$ is the systemic velocity (usually including the instrumental offset). The true anomaly depends on $e$ and the eccentric anomaly $E$ :

$$
\cos f=\frac{\left(1-e^{2}\right)}{1-e \cos E}-1
$$

the eccentric anomaly, in turn, depends on $T, t_{0}$ and time $t$ in the form of the so called Kepler's equation:

$$
E-e \sin E=\frac{2 \pi}{T}\left(t-t_{0}\right)
$$

Eq. 4.1 is minimized using the Nelder-Mead algorithm implementation from lmfit (Newville et al., 2016, version 0.9.5) to obtain the best-fit orbital parameters to the observed data. Because different instruments have different instrumental offsets, the use of additional RV data from other programs require the estimation of an extra value of $\gamma$ for each instrument.

The uncertainties of the orbital parameters are estimated using emcee, an implementation of the Affine Invariant Markov chain Monte Carlo Ensemble sampler (Foreman-Mackey et al., 2013, version 2.2.1) using flat priors for all parameters in both MC10 and EXOFAST parametrizations. These routines were implemented in the Python package radial ${ }^{4}$, which is openly available online. The uncertainties in $m \sin i$ and $a$ quoted in our results already take into account the uncertainties in the stellar masses of the solar twins.

\subsubsection{Binaries with partial orbits}

For the binary systems with long periods (typically 20 years or more), it is possible that the time span of the observations does not allow for a full coverage of at least one phase of

\footnotetext{
${ }^{4}$ Available at https://github.com/RogueAstro/radial.
} 
the orbital motion. In these cases, the estimation of the orbital parameters renders a number of possible solutions, which precludes us from firmly constraining the configuration of the system. Nevertheless, RV data containing a curvature or one inflection allows us to place lower limits on $K, T$ and $m \sin i$, whilst leaving $e$ and $\omega$ completely unconstrained. When the RV data are limited but comprise two inflections, it may be possible to use the methods from Section 4.4.1 to constrain the orbital parameters, albeit with large uncertainties.

For stars with very large orbital periods $(T \gtrsim 100 \mathrm{yr})$, the variation of radial velocities may be present in the form of a simple linear trend. In these cases, it is still possible to obtain an estimate of the mass of the companion - a valuable piece of information about it: Torres (1999) describes a statistical approach to extract the sub-stellar companion mass when the only information available from radial velocities is the inclination of the linear trend, provided information about the angular separation of the system is also available. In this approach, we need to adopt reasonable prior probability density functions (PDF) for the eccentricity $e$, the longitude of periastron $\varpi$, phase $\phi$ and the inclination $i$ of the orbital plane. As in Torres, we adopt the following PDFs: $p(i)=\sin i, p(e)=2 e$ and flat distributions for $\varpi$ and $\phi$.

We sample the PDFs using emcee, 20 walkers and 10000 steps; the first 500 burn-in steps are discarded. From these samples, we compute the corresponding companion masses and its posterior distribution. This posterior usually displays a very strong peak and long tails towards low and high masses which can be attributed to highly unlikely orbital parameters (see Fig. 4.6 for an example). In our results, we consider that the best estimates for the companion masses are the central bin of the highest peak of the distribution in a histogram with log-space bin widths of about $0.145 \operatorname{dex}\left(\mathrm{M}_{\odot}\right)$.

When no adaptive optics ( $\mathrm{AO})$ imaging data are available for the stars with a linear trend in their RVs, the most conservative approach is to provide the minimum mass for the putative companion. In the case of a linear trend, the lowest mass is produced when $e=0.5, \omega=\pi / 2$ and $\sin i=1$ (Feng et al., 2015), yielding

$$
m_{\min } \approx\left(0.0164 \mathrm{M}_{\mathrm{Jup}}\right)\left(\frac{\tau}{\mathrm{yr}}\right)^{4 / 3}\left|\frac{d v / d t}{\mathrm{~m} \mathrm{~s}^{-1} \mathrm{yr}^{-1}}\right|\left(\frac{M}{\mathrm{M}_{\odot}}\right)^{2 / 3},
$$

where $\tau$ is 1.25 multiplied by the time span of the radial velocities and $d v / d t$ is the inclination of the linear trend. 


\subsection{Results}

We discovered new, short-period companions for the stars HIP 6407 and HIP 30037 (see Fig. 4.1) and new long-period companions for HIP 54582 and HIP 62039, and updated or reproduced the parameters of several other known binaries that were observed in our program (see Figs. 4.2 and 4.4). We briefly discuss below each star, pointing out the most interesting results, inconsistencies and questions that are still open about each of them. The orbital parameters of the binaries with well-sampled orbits in their RV data are presented in Table 4.2 and the systems with partial orbits are reported in Tables 4.3 and 4.4.

\subsubsection{Withdrawn binary candidates}

In Paper IV, we showed that HIP 43297 had a rotational velocity $v \sin i$ higher than expected for its age. Moreover, its radial velocities had variations that hinted for one or more companions orbiting it. We carefully analyzed the RVs and concluded that the periodic $(T=3.8 \mathrm{yr})$ signal observed is highly correlated (Pearson $R=0.893$ ) with the activity $S$-index of the star (Freitas et al., 2017). In addition, we tentatively fitted a linear trend to the combined RVs from HARPS, ELODIE and SOPHIE, and obtained an inclination of $4.53 \pm 0.04 \mathrm{~m} \mathrm{~s}^{-1} \mathrm{yr}^{-1}$, but further monitoring of the system is required to infer the presence of a long-period spectroscopic companion. The revised stellar age for HIP 43297 yields $1.85 \pm 0.50 \mathrm{Gyr}$ (Spina et al., in preparation), which explains the high rotational velocity and activity.

The solar twin HIP 64673 displays significant fluctuations in its radial velocities, but they do not correlate with its activity index; the data covers approximately 5 years of RV monitoring and displays an amplitude $>20 \mathrm{~m} \mathrm{~s}^{-1}$. If confirmed to be caused by massive companions, the RV variations of both HIP 43297 and HIP 64673 suggest substellar masses for the most likely orbital configurations. These stars are, thus, removed from the binaries sample of the Solar Twin Planet Search program.

\subsubsection{Solar twins with new companions}

HIP 6407: This is a known binary system located $58 \mathrm{pc}$ away from the solar system (van Leeuwen, 2007), possessing a very low-mass $\left(0.073 \mathrm{M}_{\odot}\right)$ L2-type companion separated by $44.8^{\prime \prime}$ (2222 AU), as reported by Baron et al. (2015, and references therein). In this study, we report the detection of a new close-in low-mass companion with $m \sin i=0.12 \mathrm{M}_{\odot}$ on a very eccentric 

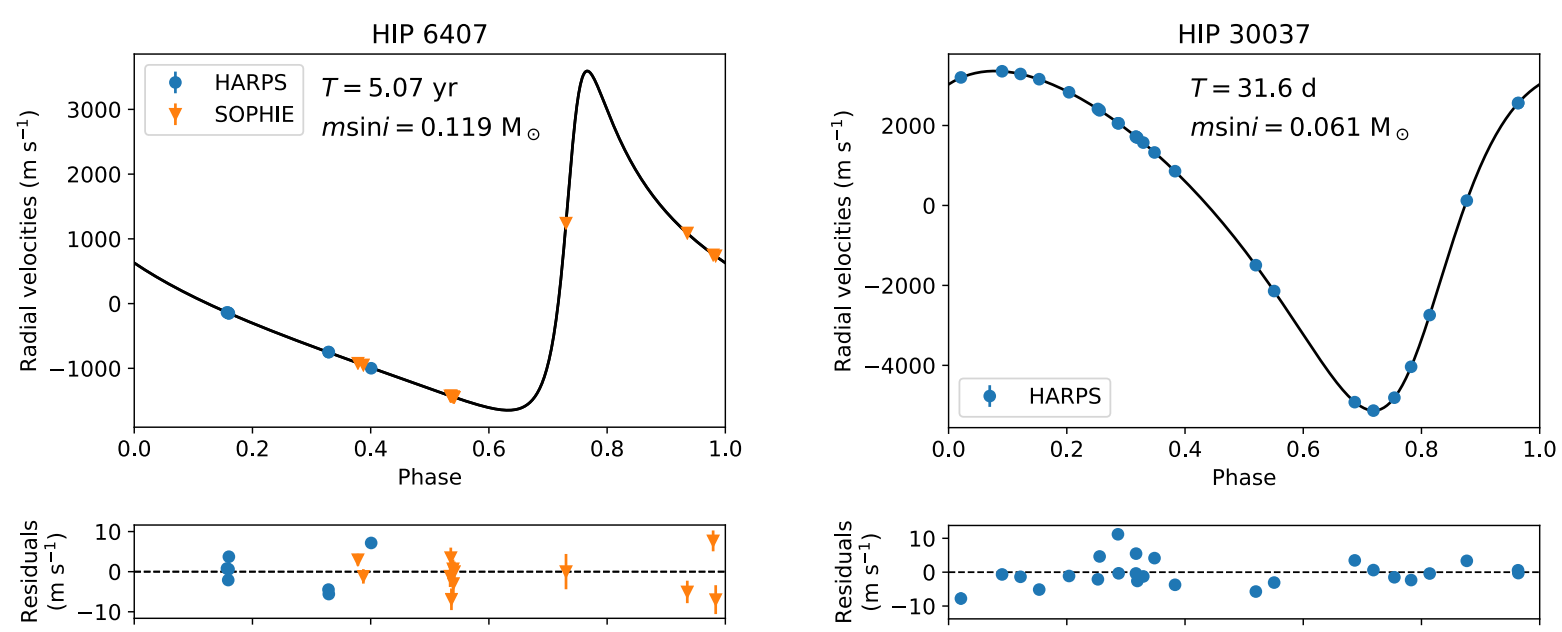

Figure 4.1: The radial velocities and orbital solutions of the newly discovered companions for solar twins with short orbital periods.

Table 4.2 - Orbital parameters of the binaries with complete or near-complete orbital phases in their RV data.

\begin{tabular}{|c|c|c|c|c|c|c|c|c|}
\hline HIP & HD & $\begin{array}{c}K \\
\left(\mathrm{~km} \mathrm{~s}^{-1}\right)\end{array}$ & $\begin{array}{c}T \\
\text { (days) }\end{array}$ & $\begin{array}{c}t_{0} \\
\text { (JD-2.45E6 days) }\end{array}$ & $\begin{array}{c}\omega \\
o\end{array}$ & $e$ & $\begin{array}{c}m \sin i \\
\left(\mathrm{M}_{\odot}\right)\end{array}$ & $\begin{array}{c}a \\
(\mathrm{AU})\end{array}$ \\
\hline \multirow{2}{*}{6407} & \multirow{2}{*}{8291} & 2.614 & 1852.3 & 5076.7 & -57.1 & 0.682 & 0.119 & 3.070 \\
\hline & & \pm 0.084 & $\begin{array}{r}+3.3 \\
-3.1 \\
\end{array}$ & $\begin{array}{r}+1.1 \\
-1.3 \\
\end{array}$ & \pm 0.9 & $\begin{array}{l}+0.009 \\
-0.010 \\
\end{array}$ & \pm 0.002 & \pm 0.005 \\
\hline \multirow{2}{*}{19911} & \multirow{2}{*}{26990} & 7.707 & 2074.15 & 4627.4 & 40.92 & 0.8188 & 0.313 & 6.16 \\
\hline & & \pm 0.007 & \pm 0.09 & \pm 0.1 & \pm 0.06 & \pm 0.0003 & \pm 0.002 & \pm 0.02 \\
\hline \multirow{2}{*}{30037} & \multirow{2}{*}{45021} & 4.246 & 31.61112 & 5999.413 & -133.60 & 0.30205 & 0.0610 & 0.1971 \\
\hline & & \pm 0.003 & \pm 0.00006 & \pm 0.001 & \pm 0.02 & \pm 0.00008 & \pm 0.0002 & \pm 0.0003 \\
\hline \multirow{2}{*}{65708} & \multirow{2}{*}{117126} & 5.754 & 207.273 & -3675.8 & -137.9 & 0.311 & 0.170 & 0.851 \\
\hline & & \pm 0.007 & \pm 0.004 & \pm 0.3 & \pm 0.2 & \pm 0.002 & \pm 0.001 & \pm 0.001 \\
\hline \multirow{2}{*}{67620} & \multirow{2}{*}{120690} & 6.311 & 3803.3 & 4945.7 & 145.10 & 0.3428 & 0.578 & 5.50 \\
\hline & & \pm 0.002 & \pm 0.4 & \pm 0.7 & \pm 0.07 & \pm 0.0002 & \pm 0.002 & \pm 0.01 \\
\hline \multirow{2}{*}{79578} & \multirow{2}{*}{145825} & 1.125 & 6681.8 & 879.2 & 138.9 & 0.3322 & 0.1014 & 7.216 \\
\hline & & \pm 0.007 & \pm 1.5 & \pm 1.9 & \pm 0.1 & \pm 0.0003 & \pm 0.0002 & \pm 0.007 \\
\hline \multirow{2}{*}{81746} & \multirow{2}{*}{150248} & 1.987 & 3246.5 & 5623.8 & -2.49 & 0.6644 & 0.1079 & 4.387 \\
\hline & & \pm 0.001 & \pm 0.7 & \pm 0.6 & \pm 0.06 & \pm 0.0005 & \pm 0.0002 & \pm 0.003 \\
\hline \multirow{2}{*}{103983} & \multirow{2}{*}{200565} & 2.100 & 10278 & 6659 & -51.6 & 0.50 & 0.210 & 9.8 \\
\hline & & \pm 0.018 & $\begin{array}{l}+274 \\
-247 \\
\end{array}$ & \pm 11 & \pm 1.5 & \pm 0.01 & \pm 0.005 & \pm 0.2 \\
\hline
\end{tabular}

orbit ( $e=0.67$ ) with $a=3 \mathrm{AU}$ and an orbital period of approximately 5 years. As expected, the long-period companion does not appear in the RV data as a linear trend.

HIP 30037: The most compact binary system in our sample, hosting a brown dwarf companion orbiting the main star with a period of 31 days. The high precision of its parameters owes to the wide time span of observations, which covered several orbits. This is one of the first 

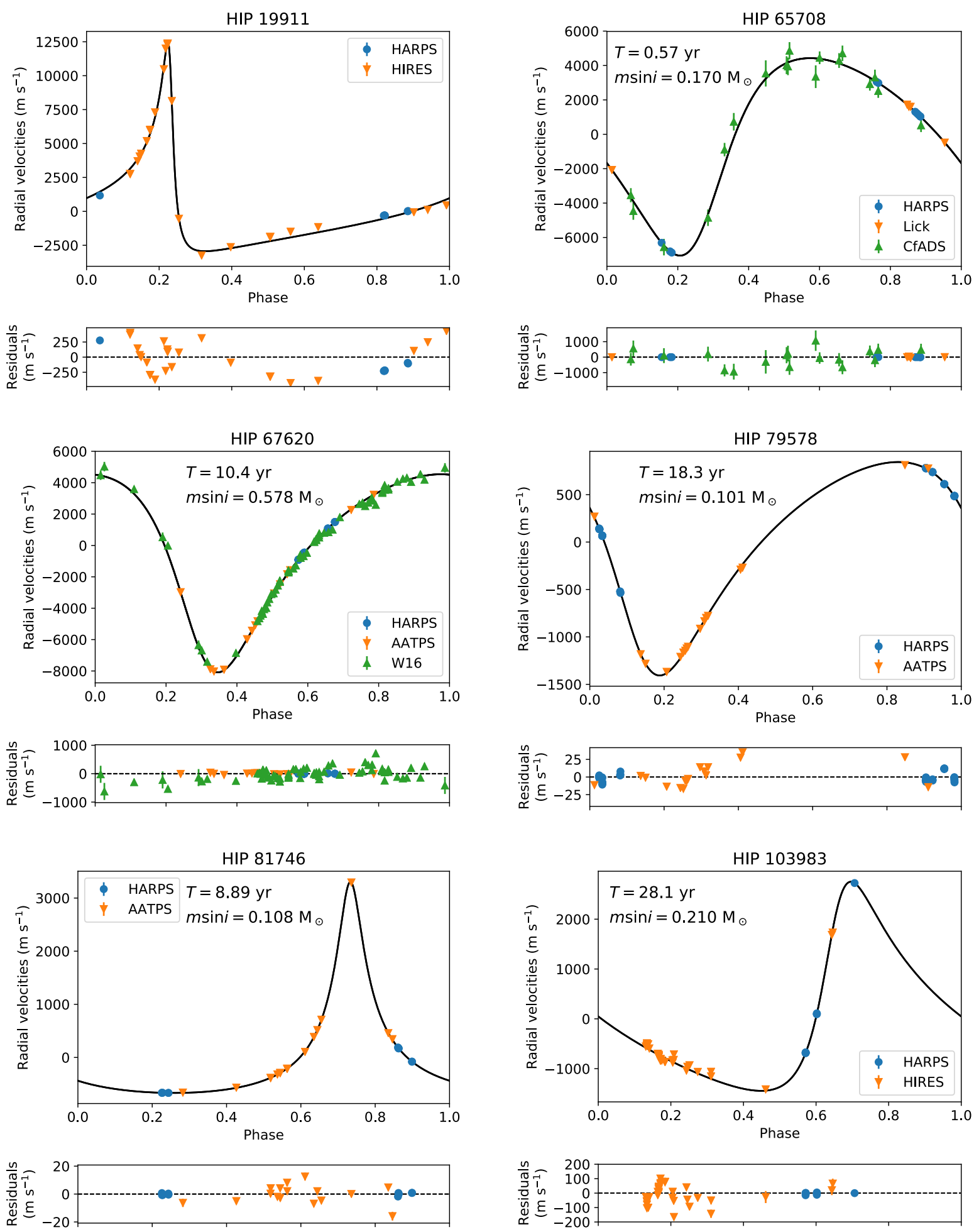

Figure 4.2: The radial velocities and updated orbital solutions of the known solar twin binaries with at least one complete or near-complete orbital phase. The acronyms CfADS, W16 and AATPS correspond to data from, respectively, the Center for Astrophysics Digital Speedometers, Willmarth et al. (2016) and the Anglo-Australian Telescope Planet Search program. 
detections of a close-in brown dwarf orbiting a confirmed solar twin ${ }^{5}$. HIP 30037 is a very quiet star, displaying no excessive correlated noise in its radial velocities. We ran stellar evolution models with MESA ${ }^{6}$ (Paxton et al., 2011, 2015) to test the hypothesis of the influence of tidal acceleration caused by the companion on a tight orbit, and found that, for the mass and period of the companion, we should expect no influence in the rotational velocity.

HIP 54582: $R V$ Curvature only. There are no reports of binarity in the literature. The slight curvature in the RVs of this star is only visible when we combine the HARPS data and the Lick Planet Search archival data. Owing to the absence of an inflection point, the orbital parameters of this system are highly unconstrained. We found that an orbit with $e \approx 0.2$ produces the least massive companion and shortest orbital period $\left(m \sin i=0.03 \mathrm{M}_{\odot}\right.$ and $\left.T=102 \mathrm{yr}\right)$.

HIP 62039: Linear trend. There are no reports of visually detected close-in $\left(\rho<2^{\prime \prime}\right)$ companions around it. This can be attributed to: i) low luminosity companion, which is possible if it is a white dwarf or a giant planet, and ii) unfavorable longitude of periapse during the observation windows. By using Eq. 4.5, we estimate that the minimum mass of the companion is $19 \mathrm{M}_{\mathrm{Jup}}$.

\subsubsection{The peculiar binaries}

\subsubsection{HIP 19911}

This is one of the main outlier stars in the overall sample of solar twins in regards to its rotation and activity, which are visibly enhanced for both the previous and revised ages (Paper IV; Freitas et al. 2017; Spina et al., in preparation). For the estimation of orbital parameters reported below, we used only the LCES HIRES/Keck radial velocities, because there are too few HARPS data points to justify the introduction of an extra source of uncertainties (the HARPS points are, however, plotted in Fig. 4.2 for reference). When using the HARPS data, although the solution changes slightly, our conclusions about the system remain the same.

The orbital solution of HIP 19911 renders a $0.31 \mathrm{M}_{\odot}$ companion in a highly eccentric orbit ( $e=0.82$, the highest in our sample), with period $T=5.7 \mathrm{yr}$. Visual scrutiny reveals what seems to be another signal with large amplitude in the residuals of this fit (>250 $\mathrm{m} \mathrm{s}^{-1}$, see Fig. 4.2); the periodogram of the residuals shows a very clear peak near the orbital period of the stellar companion.

\footnotetext{
${ }^{5}$ There are at least 4 solar twin candidates with a close-in brown dwarf companion listed in table A.1 in Wilson et al. (2016).

${ }^{6}$ Modules for Experiments in Stellar Astrophysics, available at http: //mesa. sourceforge . net
} 

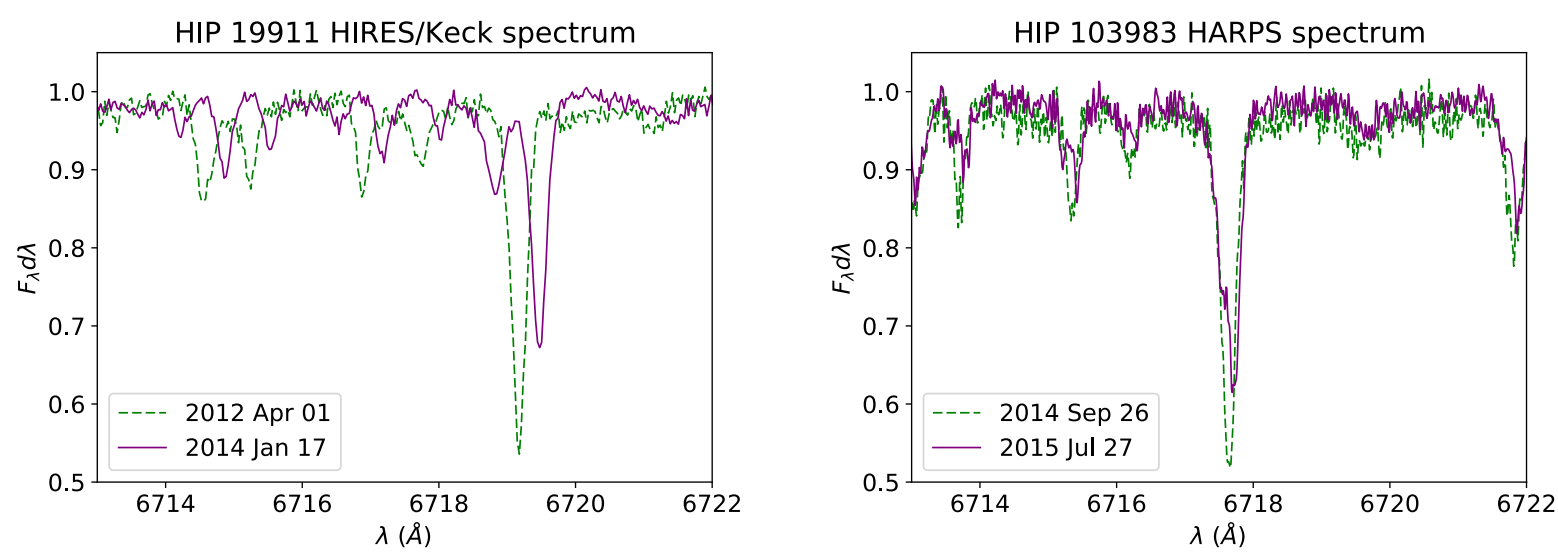

Figure 4.3: The spectrum of HIP 19911 and HIP 103983 are contaminated by bright companions. The double-lined nature of these stars is only clear only in the maximum RV separation, which are the solid purple curves. The dashed green curves show spectra during minimum RV separation where there is no obvious contamination. The spectra in this figure are not Doppler-corrected for their systemic velocities or instrumental RV offset.

The cross-correlation function (CCF) plots for the HARPS spectra of HIP 19911 display a significant asymmetry - longer tail in the blue side - for the observations between October 2011 and February 2012, which suggests that the companion is contaminating the spectra. Upon visual inspection of the archival HIRES spectra ${ }^{7}$ taken on 17 January 2014, which is when we expect the largest RV difference between the main star and its companion, we saw a clear contamination of the spectrum by the companion (see Fig. 4.3). This contamination could explain the large residuals of the orbital solution, as it introduces noise to the measured radial velocities. The double-lines also explain the inferred high rotational velocity of HIP 19911, since they introduce extra broadening to the spectral lines used to measure rotation. The presence of a bright companion may also affect estimates of chemical abundances, which elucidates the yttrium abundance anomaly (Paper III). The double-lined nature of this system is not observed on the HARPS spectra due to an unfavorable observation window.

Even at the largest RV separation, we did not detect the Li I line at $6707.75 \AA$ in the HIRES spectrum of the companion. This is expected because M dwarf stars have deeper convection zones, which means they deplete lithium much faster than Sun-like stars. This leads us to conclude that estimates of Li abundance on solar twin binaries using this line do not suffer from strong contamination by their companions; consequently, age estimates with lithium abundances may be more reliable for such binaries than isochronal or gyro ages.

Another observation conundrum for this system is that Riddle et al. (2015), using AO imaging

\footnotetext{
${ }^{7}$ Available at http://nexsci.caltech.edu/archives/koa/.
} 
without a coronagraph, reports the detection of a visual companion with orbital period $\sim 12.4 \mathrm{yr}$ (roughly twice the one we estimated), a lower eccentricity $(e=0.1677)$ and similar semi-major axis of the orbit ( $a=6.17 \mathrm{AU}$, if we consider a distance of $30.6 \mathrm{pc}$ ). Moreover, Tokovinin (2014a, hereafter T14) reports that this visual companion has $m=0.85 \mathrm{M}_{\odot}$. The most likely explanation is that the observations of Riddle et al. did in fact detect the spectroscopic companion, but the coarse timing of the observations produced a larger period; the lower eccentricity could be explained by a strong covariance between $e$ and the inclination $i$. If $i$ is lower, that means the mass of the companion is significantly higher than $m \sin i=0.316 \mathrm{M}_{\odot}$, and that would explain the value obtained by T14. A companion with a mass as large as $0.85 \mathrm{M}_{\odot}$ would likely pollute the spectra of HIP 19911, which agrees with our observation that this is a SB II system. If confirmed, this prominent $\sim 0.85 \mathrm{M}_{\odot}$ red dwarf companion could explain the observed activity levels for HIP 19911, since red dwarf stars are expected to be more active than Sun-like stars.

\subsubsection{HIP 67620}

This is a well-known binary and the target with the largest amount of RV data available (see Fig. 4.2). Its orbital parameters have been previously determined by Abt and Willmarth (2006) and more recently by Jenkins et al. (2015) and Willmarth et al. (2016). The orbital parameters we obtained are in good agreement with Willmarth et al. (2016). It has one of the most peculiar rotation rates from our sample $\left(2.77 \mathrm{~km} \mathrm{~s}^{-1}\right.$ for an age of $\left.7.18 \mathrm{Gyr}\right)$, an enhanced chromospheric activity (Freitas et al., 2017) and an anomalous [Y/Mg] abundance (Paper III). The orbital period of the system is far too long for gravitational interaction to enhance the rotation of the main star through tidal acceleration, thus we should expect that they evolve similarly to single stars from this point of view.

High-resolution imaging of HIP 67620 revealed a companion with $V_{\mathrm{mag}} \approx 10$ and separations which are consistent with the spectroscopic companion (Hartkopf et al. 2012; T14). As explained by Jenkins et al. (2015), the presence of a companion with $m>0.55 \mathrm{M}_{\odot}$ can produce contaminations to the spectra that introduce noise to the measured RVs; our estimate of $m \sin i$ for this system is $0.58 \mathrm{M}_{\odot}$. These results suggest that, similarly to HIP 19911 but to a lesser degree, the companion of HIP 67620 may be offsetting our estimates for rotational velocity, stellar activity, chemical abundances and isochronal age.

We were unable to discern double-lines in the HARPS spectra, likely resulting from unfavorable Doppler separations (observations range from February 2012 to March 2013). However, 
an analysis of the CCF of this star shows slight asymmetries in the line profiles of the HARPS spectra, which indicates a possible contamination by the companion. Fuhrmann et al. (2017) reported HIP 67620 as a double-lined binary using spectra taken at high-resolution $(R \approx 60,000)$ in February 2014 and July 2015. As expected due to the short time coverage of the HARPS spectra, we did not see any correlation between the bisector inverse slope (as defined in Queloz et al., 2001) and the radial velocities of HIP 67620.

Jenkins et al. (2015) found an additional signal on the periodogram of HIP 67620 at 532 $\mathrm{d}$, which could be fit with a $1 \mathrm{M}_{\mathrm{Jup}}$ planet, bringing down the rms of the fit by a factor of 2 . However, we did not find any significant peak in the periodogram of the residuals of the radial velocities for HIP 67620.

\subsubsection{HIP 103983}

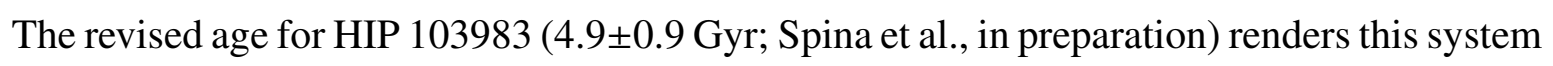
as an abnormally fast rotator $\left(3.38 \mathrm{~km} \mathrm{~s}^{-1}\right)$ for its age. However, upon a careful inspection of the HARPS data obtained at different dates, we identified that the spectrum from 2015 July 27 displays clearly visible double-lines, albeit not as well separated as those observed in the HIRES spectra of HIP 19911 (see Fig. 4.3). No other anomalies besides enhanced rotation were inferred for this system. The CCF plots of the HARPS spectra show clear longer tails towards the blue side for most observations.

In Paper IV we reported distortions in the combined spectra of HIP 103983; this is likely a result from the combination of the spectra at orbital phases in which the Doppler separation between the binaries is large. Since the observing windows of the HARPS spectra of HIP 19911 and HIP 67620 do not cover large RV separations (see Fig. 4.2), the same effect is not seen in the combined spectra of these stars. This effect also explains why HIP 103983 is an outlier in fig. 4 of Paper IV.

Although we have limited RV data, the emcee simulations converge towards a well-defined solution instead of allowing longer periods, as these produce larger residuals. It is important, however, to keep monitoring the radial velocities of this system in order to confirm that the most recent data points are in fact a second inflection in the radial velocities. The residuals for the fit for the HIRES spectra are on the order of $100 \mathrm{~m} \mathrm{~s}^{-1}$, which is likely a result from the contamination by a bright companion. $\mathrm{T} 14$ reported a $0.91 \mathrm{M}_{\odot}$ visual companion at a separation of $0.093^{\prime \prime}$, which is consistent with the spectrocopic semi-major axis we estimated: $0.149^{\prime \prime}$ for a 
distance of 65.7 pc (van Leeuwen, 2007).

\subsubsection{Other binaries with updated orbital parameters}

Among the known binaries in the solar twins sample, five of them display curvature in their RV data which allows the estimation of limits for their orbital parameters (see Table 4.3 and Fig. 4.4). Some of the linear trend binaries observed in our HARPS Solar Twin Planet Search program are targets with large potential for follow-up studies. For the companions with visual detection, we were able to estimate their most likely mass (see Table 4.4).

HIP 14501: Linear trend. Its companion is reported by Crepp et al. (2014) as the first directly imaged $\mathrm{T}$ dwarf that produces a measurable doppler acceleration in the primary star. Using a low-resolution direct spectrum of the companion, Crepp et al. (2015) estimated a modeldependent mass of $56.7 \mathrm{M}_{\text {Jup }}$. Using the HARPS and HIRES/Keck RV data and the observed separation of 1.653" (Crepp et al., 2014), we found that the most likely value of the companion mass is $0.043 \mathrm{M}_{\odot}\left(45 \mathrm{M}_{\text {Jup }}\right)$, which agrees with the mass obtained by Crepp et al. (2015). The most recent HARPS data hints of an inflection point in the orbit of HIP 14501 B (see Fig. 4.5), but further RV monitoring of the system is necessary to confirm it.

HIP 18844: Linear trend. It is listed as a multiple system containing a closer-in low-mass stellar companion (estimated $0.06 \mathrm{M} \odot$, which agrees with our most likely mass) and orbital period $T=6.5 \mathrm{yr}$ (T14, and references therein). For the companion farther away, Jenkins et al. (2015) reported a minimum orbital period of $\sim 195 \mathrm{yr}$ and $m \sin i=0.33 \mathrm{M}_{\odot}$, with a separation of $29^{\prime \prime}$ in 1941 ( 750 AU for a distance of $\left.26 \mathrm{pc}\right)$.

HIP 54102: $R V$ curvature only. It is listed as a proper motion binary by Makarov and Kaplan (2005), but there are no other information about the companions in the literature. Its eccentricity is completely unconstrained due to lack of RV coverage. We estimate that its companion's minimum mass is $12.6 \mathrm{M}_{\mathrm{Jup}}$, with an orbital period larger than 14 years.

HIP 64150: Linear trend. The most likely companion mass obtained by the method explained in Section 4.4.2 renders an estimate of $0.26 \mathrm{M}_{\odot}$, as seen in Fig. 4.6. The higher mass $(0.54$ $\mathrm{M}_{\odot}$ ) obtained by Crepp et al. 2013 and M14 can be attributed to less likely orbital configurations, but it is still inside the $1-\sigma$ confidence interval of the RV+imaging mass estimate. The main star displays clear signals of atmosphere pollution caused by mass transfer from its companion during the red giant phase (Takeda et al., 2011), characterizing the only confirmed blue straggler of our sample. The measured projected separation of the binary system is 18.1 AU (M14), which 

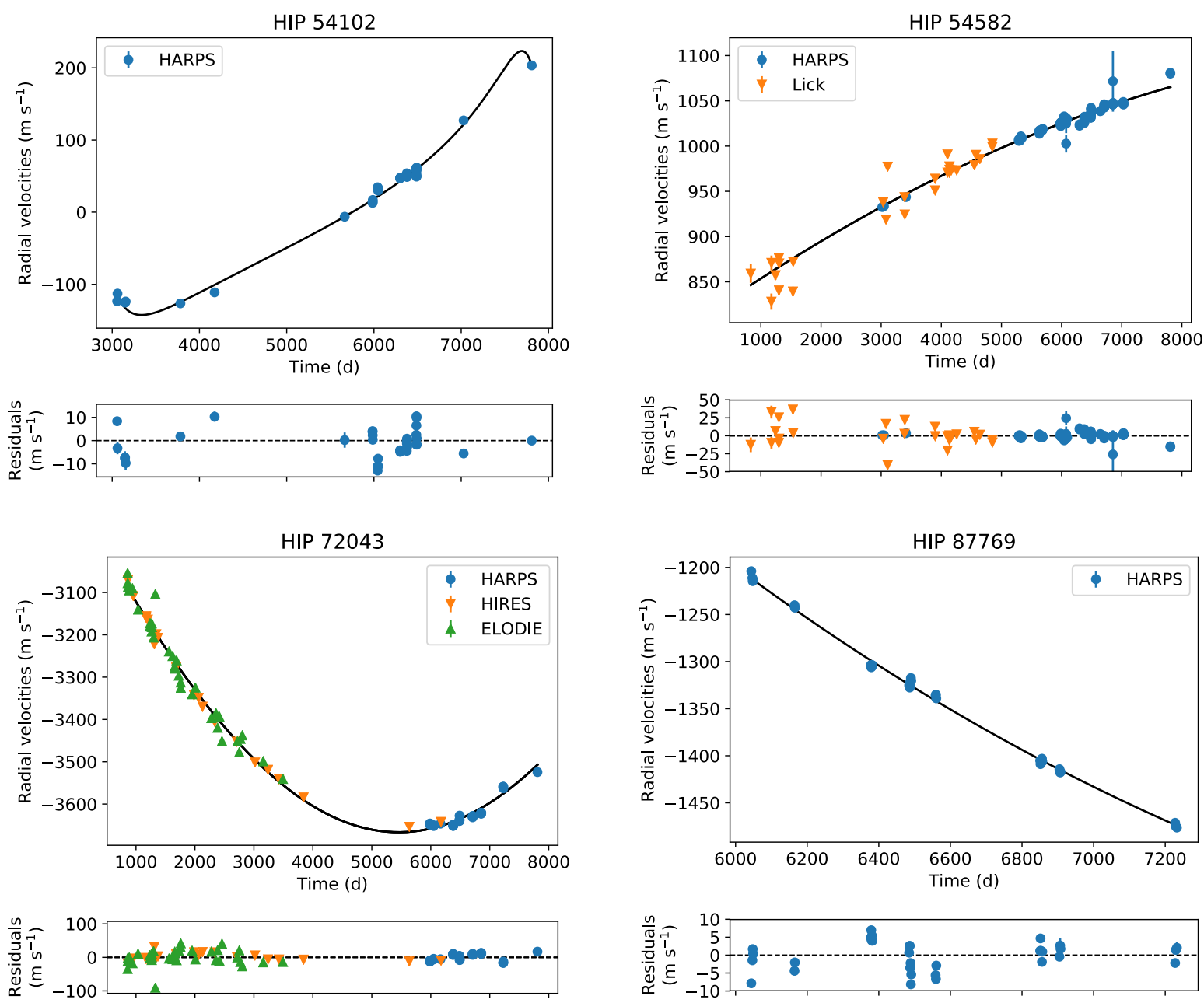

Figure 4.4: The radial velocities and the solutions that produced the lower limits of orbital parameters of the solar twin binaries with RV curvature. A similar plot for HIP 73241 can be found in Jenkins et al. (2015). Time is given in JD $-2.45 \times 10^{6} \mathrm{~d}$.

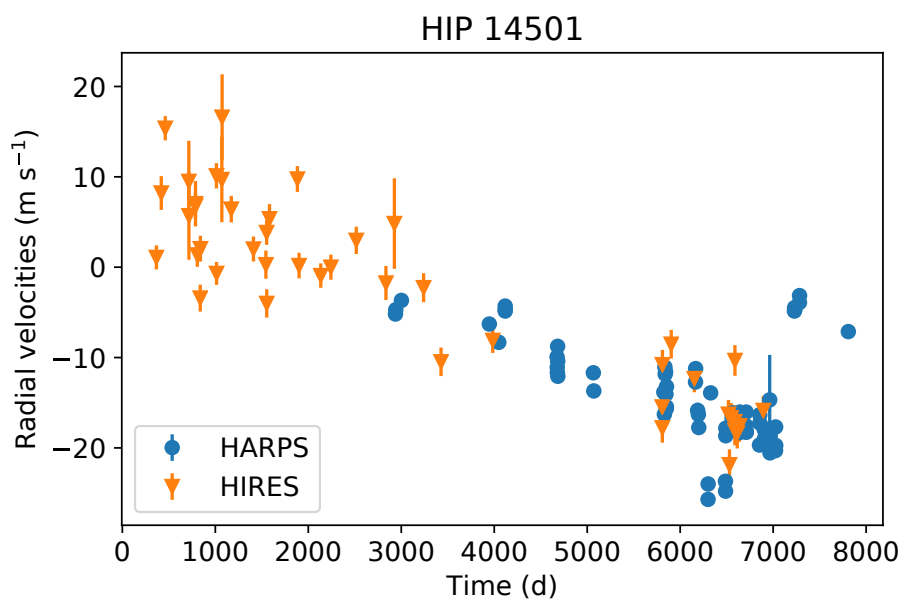

Figure 4.5: Radial velocities of HIP 14501. The RV shift in the $y$-axes is arbitrary. Time is given in JD $-2.45 \times 10^{6}$ d. 


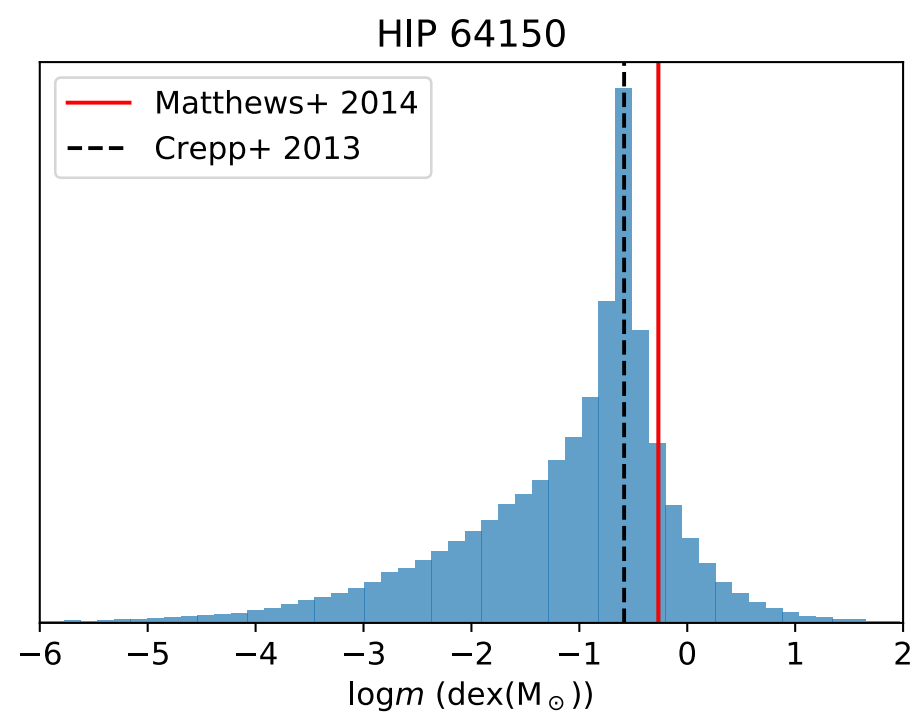

Figure 4.6: Posterior probability distribution of the companion mass for HIP 64150. The mass obtained by Matthews et al. (2014) using SED fitting for the spectra of the WD companion is shown as a red vertical line.

indicates that even for such a wide system the amount of mass transferred is still large enough to produce measurable differences in chemical abundances. It seems, however, that the amount of angular momentum transfer was not enough to produce significant enhancement in the rotation rate and activity of the solar twin. It is also important to note that the isochronal age measured for this system (Paper III) has a better agreement with the white dwarf (WD) cooling age estimated by M14 than previous estimates, illustrating the importance of studying these Sirius-like systems to test the various methods of age estimation.

HIP 65708: This star has previously been reported as a single-lined spectroscopic binary with an orbital solution (Latham et al., 2002). Here we update this solution by leveraging the extremely precise radial velocities measured in the Lick Planet Search program and with the HARPS spectrograph. The minimum mass of the companion is $0.167 \mathrm{M}_{\odot}$, indicating it is a red dwarf, orbiting at less than $1 \mathrm{AU}$ with a slight eccentricity of 0.31 . Our results agree with the previous orbital solution, which was based solely on data with uncertainties two orders of magnitude higher than the most recent data from HARPS and the Lick Planet Search.

HIP 72043: $R V$ curvature only. Similarly to HIP 54102, it is listed as a proper motion binary and we could not constrain its eccentricity. A fairly massive $\left(>0.5 \mathrm{M}_{\odot}\right)$ companion is inferred at a very large period; this fit suggests that the longitude of periapse of the companion of HIP 72043 is currently at an unfavorable position for visual detection.

HIP 73241: $R V$ curvature only. The companion's orbit is eccentric enough to allow an 
estimation of the minimum eccentricity; its companion has been previously been confirmed by Raghavan et al. (2010) and visually detected by T14 with a separation $0.318^{\prime \prime}$. In Paper IV we listed this star as having an unusually high rotation, but here we revise this conclusion and list HIP 73241 as a candidate peculiar rotator because its $v \sin i$ is less than $2 \sigma$ above the expected value for its age. Similarly to HIP 67620, this peculiarity, if real, could also be explained by contamination by a bright companion, since we determined that the minimum companion mass $m \sin i>0.49 \mathrm{M}_{\odot}$.

HIP 79578: The companion is a well-defined $0.10 \mathrm{M}_{\odot}$ red dwarf orbiting the main star approximately every 18 years in a fairly eccentric orbit $(e=0.33)$. The orbital parameters we obtained differ significantly from the ones obtained by Jenkins et al. (2015) by more than 10\%, except for the eccentricity; also in contrast, Jenkins et al. report it as a brown dwarf companion. The fit for this binary displays residuals of up to $30 \mathrm{~m} \mathrm{~s}^{-1}$ for the AATPS radial velocities, and the periodogram of these residuals shows a peak near the period 725 days. When we fit an extra object with $m \sin i=0.70 \mathrm{M}_{\mathrm{Jup}}$ at this period ( $a=1.62 \mathrm{AU}$ and $e=0.87$ ), it improves the general fit of the RVs by a factor of 7. It is important to mention, however, that there are only 17 data points for the AATPS dataset, and the HARPS dataset does not display large residuals for a single companion fit. We need thus more observations to securely infer the configuration of this binary system, and if it truly has an extra substellar companion at a shorter period.

HIP 81746: This is another high-eccentricity $(e=0.7)$ binary that does not display clear anomalies in its rotation and activity. Its companion is a $0.1 \mathrm{M}_{\odot}$ red dwarf orbiting the main star every 9 years. The orbital parameters we obtained are in good agreement with the ones reported by Jenkins et al. (2015).

HIP 83276: $R V$ curvature only. Although the HARPS radial velocities suggest the presence of a stellar mass companion, we do not have enough RV data points to infer any information about the orbital parameters of the system. Using radial velocities measured with the CORAVEL spectrograph, Duquennoy and Mayor (1991) found the companion has $m \sin i=0.24 \mathrm{M}_{\odot}, e=$ 0.185 and an orbital period of 386.72 days.

HIP 87769: $R V$ curvature only. It is reported as a binary system by T14 but, similarly to HIP 54102, lacks an inflection point in its RV data from HARPS, which spans $3.3 \mathrm{yr}$. There is a wide range of possible orbital solutions that suggest $m \sin i$ varying from brown dwarf masses to $\sim 1 \mathrm{M}_{\odot}$. Higher eccentricities $(e>0.8)$ can be ruled out as unlikely because they suggest a companion with $m \sin i \approx 1 \mathrm{M}_{\odot}$ at an orbital period of more than $500 \mathrm{yr}$ and $a>80 \mathrm{AU}$. 
Table 4.3 - Lower limits of the orbital parameters of the spectroscopic binaries with curvature in their RV data.

\begin{tabular}{llcccc}
\hline HIP & HD & $\begin{array}{c}K \\
\left(\mathrm{~km} \mathrm{~s}^{-1}\right)\end{array}$ & $\begin{array}{c}T \\
(\mathrm{yr})\end{array}$ & $\begin{array}{c}m \sin i \\
\left(\mathrm{M}_{\odot}\right)\end{array}$ & $e$ \\
\hline 54102 & 96116 & $>0.182$ & $>14$ & $>0.012$ & $\ldots$ \\
54582 & 97037 & $>0.193$ & $>102$ & $>0.03$ & $\ldots$ \\
72043 & 129814 & $>2.11$ & $>104$ & $>0.40$ & $\ldots$ \\
73241 & 131923 & $>5.93$ & $>21.0$ & $>0.49$ & $>0.72$ \\
87769 & 163441 & $>1.90$ & $>81.5$ & $>0.30$ & $\ldots$ \\
\hline
\end{tabular}

Table 4.4 - Measured RV slopes of the linear trend binaries. The most likely mass for the spectroscopic companion is estimated when their separation is available. Otherwise a minimum mass is provided.

\begin{tabular}{llcccc}
\hline HIP & HD & $\begin{array}{c}d v_{r} / d t \\
\left(\mathrm{~m} \mathrm{~s}^{-1} \mathrm{yr}^{-1}\right)\end{array}$ & $\begin{array}{c}\rho \\
(\operatorname{arcsec})\end{array}$ & $\begin{array}{c}\text { Dist. } \\
(\mathrm{pc})^{\mathrm{d}}\end{array}$ & $\begin{array}{c}m \\
\left(\mathrm{M}_{\text {Jup }}\right)\end{array}$ \\
\hline 14501 & 19467 & $-1.30 \pm 0.01$ & $1.653^{\mathrm{a}}$ & 30.86 & 45 \\
$18844^{\dagger}$ & 25874 & $424 \pm 3$ & $0.140^{\mathrm{b}}$ & 25.91 & 79 \\
62039 & 110537 & $7.25 \pm 0.03$ & $\ldots$ & 42.68 & $>19$ \\
64150 & 114174 & $61.72 \pm 0.02$ & $0.675^{\mathrm{c}}$ & 26.14 & 270 \\
\hline
\end{tabular}

\footnotetext{
${ }^{\mathrm{a}}$ Crepp et al. (2014).

${ }^{\mathrm{b}}$ Tokovinin (2014a).

${ }^{\mathrm{c}}$ Matthews et al. (2014).

$\mathrm{d}_{\text {van Leeuwen (2007). }}$.

${ }^{\dagger}$ Triple or higher-order system.
}

\subsubsection{Considerations on multiplicity statistics}

Although planet search surveys are generally biased against the presence of binaries due to avoiding known compact multiple systems, the fraction of binary or higher-order systems in the whole sample of the Solar Twin Planet Search program is $42 \% \pm 6 \%{ }^{8}$. This value agrees with previous multiplicity fractions reported by, e.g., Raghavan et al. (2010) and T14; however, it is signficantly lower than the $58 \%$ multiplicity factor for solar-type stars reported by Fuhrmann

\footnotetext{
${ }^{8}$ Counting stellar and brown dwarf companions. The uncertainty is computed using a bootstrap resampling analysis with 10,000 iterations, similarly to Raghavan et al. (2010). In each iteration, a new set of 81 solar twins is randomly drawn from the original sample allowing stars to be selected more than once.
} 


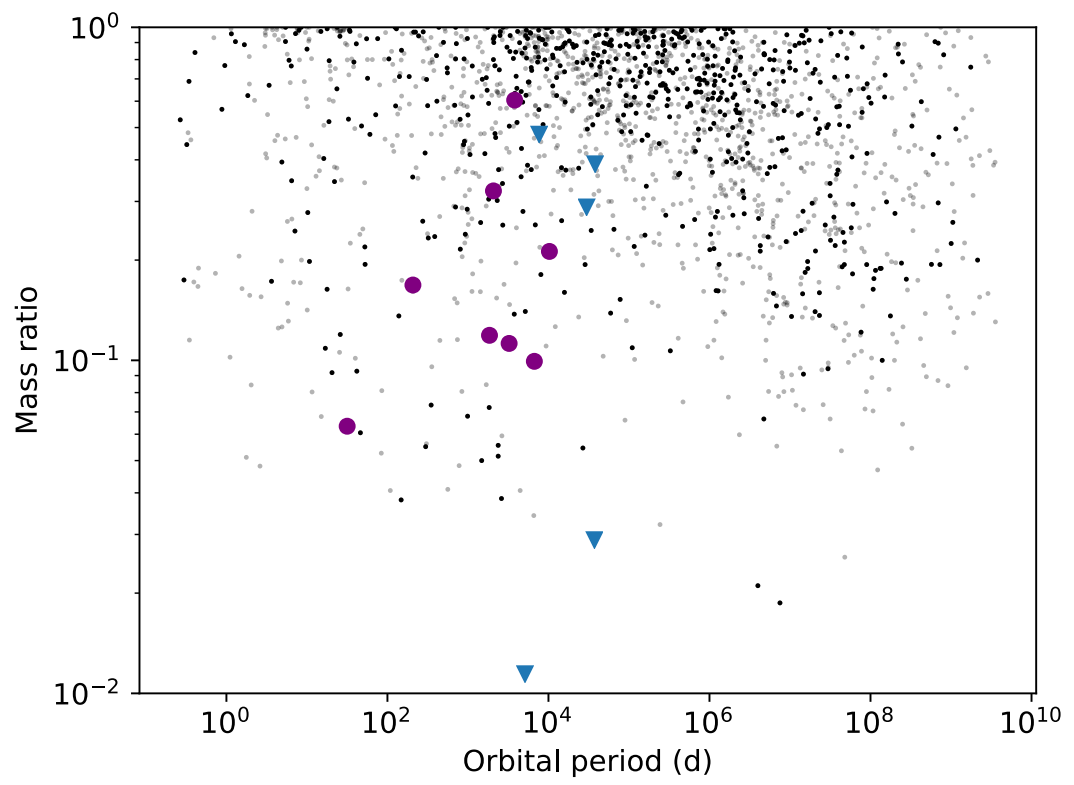

Figure 4.7: Mass ratios in function of the orbital periods of binary stars or higher-order systems in the solar neighborhood. The purple circles are binaries in our sample with well defined period and $m \sin i$; the blue triangles correspond to the binaries in our sample for which we only have lower limits for the periods and $m \sin i$. The stars from T14 are plotted as black dots (the darker ones are those with main star masses between 0.9 and $1.1 \mathrm{M}_{\odot}$ ).

et al. (2017), who argues that previous results are subject to selection effects and are thus biased against the presence of multiple systems.

The orbital period vs. mass ratio plot of companions in the Solar Twin Planet Search is shown in Fig. 4.7. A comparison with the sample of solar-type stars from T14 reveals two important biases in our sample: i) Mass ratios are mostly below 0.3 because of selection of targets that do not show large radial velocity variations in previous studies; ii) Orbital periods are mostly lower than $30 \mathrm{yr}$ because longer values cannot be constrained from the recent RV surveys targeting solar-type stars with low-mass companions. In such cases, further monitoring of linear trend and RV curvature-only binaries may prove useful to understand the origins of the brown dwarf desert (Grether and Lineweaver, 2006). These targets are particularly appealing because the long periods mean that the separation from the main star is large enough to allow us to observe them directly using high-resolution imaging.

Previous studies on the period-eccentricity relation for binary stars found that systems with orbital periods below 10 days tend to have eccentricities near zero, while those between 10 and 1000 days follow a roughly flat distribution of eccentricities (Kjurkchieva et al., 2016, and references therein), an effect that is due to the timescales for circularization of orbits. In relation to our sample, with the exception of HIP 30037, HIP 65708 and HIP 83276, all of the binaries 
we observed have periods longer than 1000 days and eccentricities higher than 0.3 , which agrees with the aforementioned findings. According to Duquennoy and Mayor (1991), the distribution of eccentricities on systems with $T>1000 \mathrm{~d}$ is a function of energy only, and does not depend on $T$ (see fig. 5 in Duquennoy and Mayor). Interestingly, HIP 30037, which hosts a brown dwarf companion with $T=31.6 \mathrm{~d}$, falls inside the 25-35 days interval of orbital periods found by Kjurkchieva et al. that corresponds to a short stage of evolution of binaries undergoing a fast change in their orbits.

\subsection{Conclusions of this chapter}

The Solar Twin Planet Search and several other programs observed 81 solar twins using the HARPS spectrograph. In total, 18 of these solar twins are spectroscopic binaries, 18 are visual binaries, and two intersect these categories. We found a multiplicity fraction of $42 \% \pm 6 \%$ in the whole sample, which is lower than the expected fraction $(\sim 58 \%)$ because of selection effects that are generally seen in exoplanet search surveys.

We updated or reproduced the solutions of several known binaries, and determined all the orbital parameters of HIP 19911, HIP 65708, HIP 67620, HIP 79578, HIP 81746 and HIP 103983. The stars HIP 43297 and HIP 64673, which we previously reported as binaries, are likely to host long-period giant planets instead of stellar companions. For binaries with partial orbits, we were able to place lower limits for some of their orbital parameters owing to the presence of curvature or an inflection point in their RV data. We estimated the most likely mass of the companions of the binaries that display only linear trends in their RV data. Future work is needed on studying the long-period binaries using photometry data and high-resolution imaging in order to constrain the nature of their companions. These wide solar twin binaries are prime targets for detailed physical characterization of their companions owing to the favorable separation for $\mathrm{AO}$ imaging and the precision with which we can measure the stellar parameters of the main star - this is particularly important for fully convective red dwarf stars and very low-mass companions such as the T dwarf HIP $14501 \mathrm{~B}$, whose evolution and structure is still poorly constrained.

Additionally, we reported the detailed discovery of new companions to the following solar twins: HIP 6407, HIP 30037, HIP 54582, and HIP 62039, for which we are able to determine an orbital solution for the first two using radial velocities. The latter two do not have enough RV data to obtain precise orbital parameters, but we can nonetheless estimate their minimum 
companions masses. We found that these new companions are likely very low-mass, ranging from 0.02 to $0.12 \mathrm{M}_{\odot}$ (although stressing that these are lower limits), which should be useful in understanding the origins of the brown dwarf desert in future research.

The anomalies and RV residuals observed on HIP 19911, HIP 67620 and HIP 103983 are likely due to contamination by the companion on the spectra of the main star. Although the peculiar stars in our sample are no longer considered blue straggler candidates, it is important to note that the detection of WD companions is particularly important for the study of field Sun-like stars because they allow the estimation of their cooling ages; these are more reliable than isochronal and chromospheric ages in some cases, providing thus robust tests for other age estimate methods. We do not expect that the presence of $\mathrm{M}$ dwarf companions contaminate lithium spectral lines in Sun-like stars, thus stellar ages derived from Li abundances may be more reliable for double-lined solar twins. We recommend a revision of the stellar parameters of the peculiar binary stars by analyzing high-resolution spectra at the highest Doppler separations possible, or using Gaussian processes to disentangle the contaminated spectra (see, e.g., Czekala et al., 2017).

We conclude that single-lined solar twin binaries with orbital periods larger than several months and moderate to low eccentricities do not display signals of distinct rotational evolution when compared to single solar twins. The most compact system in our sample, HIP 30037, which hosts a $0.06 \mathrm{M}_{\odot}$ brown dwarf companion at an orbital period of 31 days is, in fact, one of the quietest stars in the sample (in regards of its activity levels), and is thus a viable target for further efforts in detecting moderate- to long-period circumbinary planets. 


\section{Chapter 5}

\section{Concluding marks}

The Solar Twin Planet Search program was designed as an effort to procure extrasolar planets in a very particular type of stars: those that are extremely similar to the Sun (i.e., solar twins). The reason why such a narrow search makes itself necessary is because the knowledge about a newly-discovered exoplanet is limited by how well we know the host star. Since we know the Sun, or at least its stellar parameters very accurately, we can leverage this similarity to precisely and accurately infer the characteristics of the solar twins.

A by-product of this project is the hundreds of high-quality spectra for more than 70 stars in the solar neighborhood. By combining these spectra along with archival data from other programs, we are able to work with extremely high signal to noise and high resolution spectra for the solar twins. So, beyond searching for exoplanets, this data allowed us to answer different questions posed in the field of stellar evolution of Sun-like stars, particularly on their rotation. But, not surprisingly, such answers also brought along with them other questions, all of which I summarize below.

\subsection{Summary of this dissertation}

In the past, several studies were conducted to analyze how common the Sun is, but the answers were frequently conflicting. By studying strictly solar twins, we concluded that the Sun is indeed a regular star in its rotation, as seen in Chapter 3. This, associated with the discovery of exoplanets in solar twins (Bedell et al., 2015; Meléndez et al., 2017), brings to rest some previous hypotheses that proposed that the rotation of the Sun should be different because it formed a planetary system.

Another important conclusion we drew from this research is that the widely used Skumanich 
law $\left(v_{\text {rot }} \propto t^{-1 / 2}\right)$ seems to be incompatible with the rotational evolution of the solar twins. We proposed thus a new empirical relation that correctly reflects a weakened braking after solar age and a steeper decay of the rotational velocity for young Sun-like stars. This behavior is also observed in the magnetic activity evolution of these stars, and a similar conclusion can be drawn when analyzing the rotational periods of solar-type stars observed with Kepler.

The search for exoplanets around Sun-like stars generally targets single stars because they produce stable planetary systems more frequently, which improves the chance of detection. Moreover, the evolution of binary star systems is not well understood. For instance, it is usual to expect binary systems to display enhanced rotation and activity because they might have interacted with their companions at some point in their lifetimes. However, to what extent of orbital configurations and companion properties should we expect that to happen?

Several binary systems were observed in the Solar Twin Planet Search, and the high quality of the data available for them allowed us to precisely measure their physical properties and their orbital configurations. As we saw in Chapter 4, most binaries in the solar twin sample are moderately wide systems with orbital periods ranging from a few to $\sim 100$ years. On the other hand, HIP 30037 has a period of only 31 days, rendering it as the most compact system in the sample.

We showed that, with the exception of two stars (or three if we consider the most recent age estimates), all binaries in the solar twin sample seem to evolve as single stars owing to their large orbital periods. This result is important not only to impose observational constraints on theories of rotational evolution of such systems, but also to better plan targets for exoplanet searches. In selecting only single and magnetically quiet stars for such surveys, we are deemed to have incomplete statistics about the presence of planets across the spectrum of stellar properties; the extension of these surveys to include well-characterized binaries such as the ones we studied will be of crucial importance to understand the formation and evolutionary paths of plantary systems.

The only binaries with enhanced rotation and activity are HIP 19911, HIP 67620 and HIP 103983. These anomalies should only be expected for extremely compact systems, such as those with periods of only a few days, where tidal acceleration of rotation is possible. Therefore the observed anomalies in these two stars cannot solely be explained by gravitational interaction with their companions. Although the blue straggler hypothesis can explain both the enhanced rotation and the chemical anomaly, this is inconsistent with the visual detection of companions around HIP 19911 (Riddle et al., 2015) and HIP 67620 (Tokovinin, 2014a). The most likely 
explanation for the anomalies in these stars seems to be a simple contamination of its spectra by a bright companion.

\subsection{Future outlook}

\subsubsection{Rotation of Sun-like stars}

Naturally, the study of stellar rotation through spectroscopy is limited by the inclination angle $i$ of the rotational axis in relation to the plane of the sky. But, nevertheless, this limitation can be minimized if we consider that values of $\sin i$ near 1 are statistically more likely than those closer to zero, thus larger homogeneous samples of stars will address this issue. This is only possible owing to the large quantities of data being produced by high-resolution stable spectrographs such as HARPS, HARPS-N and HIRES (and, in the future, EXPRES, ESPRESS and G-CLEF).

Photometric surveys such as the ones provided by Kepler and TESS are important contributors to the study of rotation because they can effectively measure the rotational periods of a large number of stars. These periods are independent of the inclination angle $i$, but are limited in the fact that the inference of the evolution of angular momentum will be dependent on the stellar radius, and we cannot assume it to remain constant for all stellar ages, especially for old stars. In this context, notice that the flattening of the rotational velocity that we found after the solar age in fact implies increasing rotational periods (Fig. 5.1). Another problem is that photometric surveys usually target fainter stars $(V \gtrsim 10)$, and these are more difficult to characterize precisely through spectroscopy, which is a requirement to identify solar twins.

\subsubsection{Models of stellar atmospheres and evolution}

As we saw in Section 3.4.1, the estimation of macroturbulence velocities are crucial to precisely measure the rotational velocities of Sun-like stars through spectroscopy. In the future, it should be possible to calibrate macroturbulence velocities using 3D hydrodynamical stellar atmosphere models (e.g., Magic et al., 2013), by using predicted 3D line profiles (without rotational broadening) as observations and determine which value of $v_{\text {macro }}$ is needed to reproduce them with 1D model atmospheres.

With the imminent release of a vast amount of data from Gaia and more sophisticated methods to measure stellar parameters in large datasets, our knowledge of stellar evolution and its models will be put under very stringent tests. As pointed out by Dotter et al. (2017), it is 

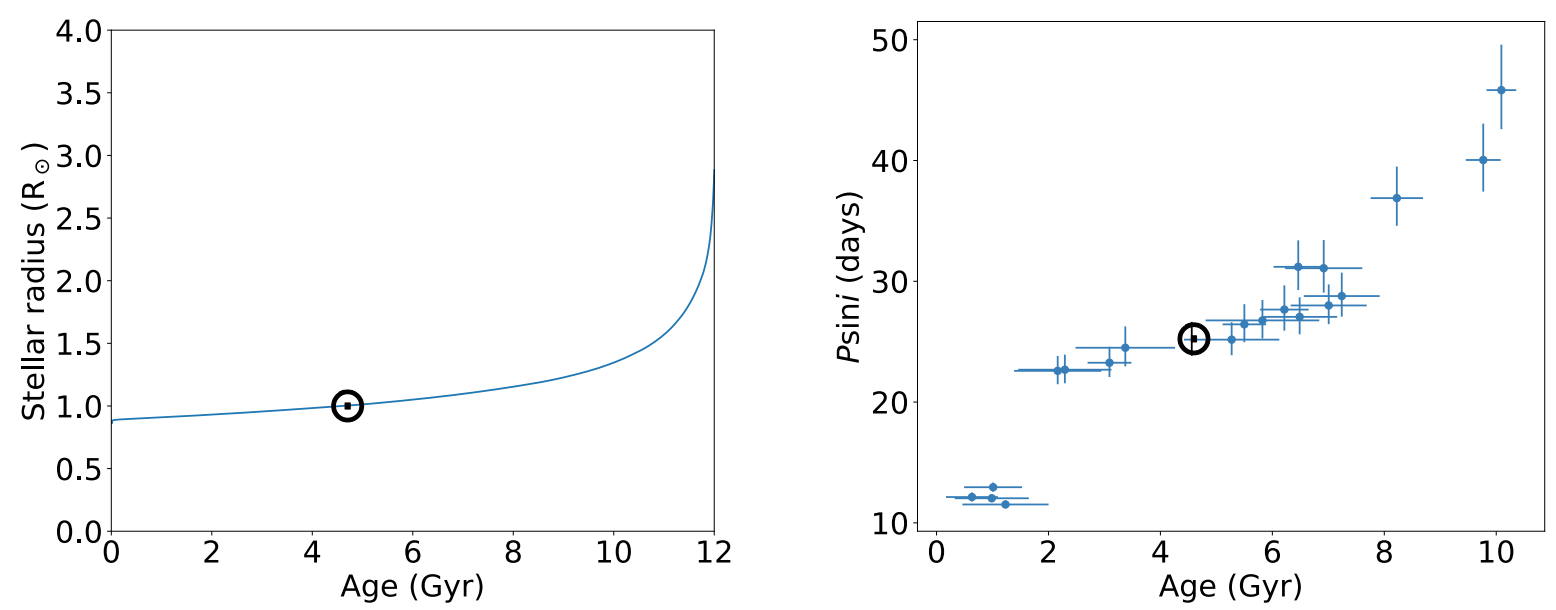

Figure 5.1: Left panel: model of the stellar radii evolution for solar twins (do Nascimento et al., 2014). Right panel: expected rotational periods $(P \sin i)$ for the solar twins using the $v \sin i$ from HARPS and the stellar radii model.

critical that we take into account the evolution of surface chemical abundances due to atomic diffusion and other mixing processes; this will be particularly important for efforts in chemical tagging and stellar age estimation.

Further research is also needed to understand the magnetic interactions between Sun-like stars and moderately close-in companions (those with orbital periods within one month and several years, such as Jupiter and the Sun). These interactions, if non-negligible, could influence the evolution of magnetism in the Sun and similar stars, and they could also play a role in the search for long-period exoplanets (see Section 5.2.3).

\subsubsection{Stellar activity and the search for habitable planets}

A natural next step for this line of research lies in the extension to studies of stellar activity of low-mass stars. Owing to the rise of the next-generation of telescopes, instruments and surveys for the detection of rocky planets, we are interested in properly modelling and taking into account the effects of stellar activity in photometric and spectroscopic methods of planet detection. Particularly for the radial velocities method, stellar activity can easily mimic the presence of rocky to giant mass planets around Sun-like stars, especially those at larger distances (see Section 4.5).

One way to take stellar activity into account when searching for exoplanets is to perform spectroscopy and photometry of the star at the same time: the first aims to measure the radial velocities, while the second is targeted towards precisely measuring the activity of the star. This 
method is, however, only fruitful for younger systems, because their main stars are still active enough to have detectable levels of variability for ground-based instruments. The resulting data will also allow us to precisely and accurately measure the physical properties and both the rotational velocities and periods of the stars.

As suggested by Haywood (2015), another pathway to improve the search for exoplanets is to trace the wavelength dependent stellar activity effects in solar-type stars. The radial velocity shifts due to planets are constant along the spectrum, but this is not true for activity. If we are able to incorporate these effects into our understanding of RV variability, it will be an important step towards detecting rocky planets in the habitability zones of solar-type stars. Also, further development of different activity indexes may help to disentangle activity from the presence of planets.

I am also interested in studying how exoplanets evolve in the presence of stellar activity. This is especially important for those found in the habitability zones of M dwarf stars (Shields et al., 2016), because these stars are much more active than solar-type ones and they will be one of the main targets of future exoplanet surveys. There are some recent studies on a few particular targets of interest (e.g., Airapetian et al., 2017; Dong et al., 2017) but, as pointed out by Bean et al. (2017), we need to cast a wider net into as many targets as we can in order to effectively answer the question of whether there is life elsewhere in the universe. 


\section{Bibliography}

Abt H. A., Willmarth D., The Secondaries of Solar-Type Primaries. I. The Radial Velocities, ApJS, 2006, vol. 162, p. 207

Airapetian V. S., Glocer A., Khazanov G. V., Loyd R. O. P., France K., Sojka J., Danchi W. C., Liemohn M. W., How Hospitable Are Space Weather Affected Habitable Zones? The Role of Ion Escape, ApJ, 2017, vol. 836, p. L3

Amard L., Palacios A., Charbonnel C., Gallet F., Bouvier J., Rotating models of young solartype stars. Exploring braking laws and angular momentum transport processes, A\&A, 2016, vol. 587, p. A105

Asplund M., Grevesse N., Sauval A. J., Scott P., The Chemical Composition of the Sun, ARA\&A, 2009, vol. 47, p. 481

Badnell N. R., Bautista M. A., Butler K., Delahaye F., Mendoza C., Palmeri P., Zeippen C. J., Seaton M. J., Updated opacities from the Opacity Project, MNRAS, 2005, vol. 360, p. 458

Bahcall J. N., Pinsonneault M. H., Basu S., Solar Models: Current Epoch and Time Dependences, Neutrinos, and Helioseismological Properties, ApJ, 2001, vol. 555, p. 990

Bahcall J. N., Pinsonneault M. H., Wasserburg G. J., Solar models with helium and heavyelement diffusion, Reviews of Modern Physics, 1995, vol. 67, p. 781

Baliunas S. L., Donahue R. A., Soon W. H., et al., Chromospheric variations in main-sequence stars, ApJ, 1995, vol. 438, p. 269

Baranne A., Mayor M., Poncet J. L., CORAVEL - A new tool for radial velocity measurements, Vistas in Astronomy, 1979, vol. 23, p. 279 
Baranne A., Queloz D., Mayor M., Adrianzyk G., Knispel G., Kohler D., Lacroix D., Meunier J.P., Rimbaud G., Vin A., ELODIE: A spectrograph for accurate radial velocity measurements., A\&AS, 1996, vol. 119, p. 373

Barnes S. A., An Assessment of the Rotation Rates of the Host Stars of Extrasolar Planets, ApJ, 2001, vol. 561, p. 1095

Barnes S. A., On the Rotational Evolution of Solar- and Late-Type Stars, Its Magnetic Origins, and the Possibility of Stellar Gyrochronology, ApJ, 2003, vol. 586, p. 464

Barnes S. A., Ages for Illustrative Field Stars Using Gyrochronology: Viability, Limitations, and Errors, ApJ, 2007, vol. 669, p. 1167

Barnes S. A., A Simple Nonlinear Model for the Rotation of Main-sequence Cool Stars. I. Introduction, Implications for Gyrochronology, and Color-Period Diagrams, ApJ, 2010, vol. 722, p. 222

Baron F., Lafrenière D., Artigau É., Doyon R., Gagné J., Davison C. L., Malo L., Robert J., Nadeau D., Reylé C., Discovery and Characterization of Wide Binary Systems with a Very Low Mass Component, ApJ, 2015, vol. 802, p. 37

Batalha N. M., Exploring exoplanet populations with NASA's Kepler Mission, Proceedings of the National Academy of Science, 2014, vol. 111, p. 12647

Baumann P., Ramírez I., Meléndez J., Asplund M., Lind K., Lithium depletion in solar-like stars: no planet connection, A\&A, 2010, vol. 519, p. A87

Bean J. L., Abbot D. S., Kempton E. M.-R., A Statistical Comparative Planetology Approach to the Hunt for Habitable Exoplanets and Life Beyond the Solar System, ApJ, 2017, vol. 841, p. L24

Bedell M., Meléndez J., Bean J. L., Ramírez I., Asplund M., Alves-Brito A., Casagrande L., Dreizler S., Monroe T., Spina L., Tucci Maia M., The Solar Twin Planet Search. II. A Jupiter twin around a solar twin, A\&A, 2015, vol. 581, p. A34

Bedell M., Meléndez J., Bean J. L., Ramírez I., Leite P., Asplund M., Stellar Chemical Abundances: In Pursuit of the Highest Achievable Precision, ApJ, 2014, vol. 795, p. 23 
Bergeron P., Wesemael F., Dufour P., Beauchamp A., Hunter C., Saffer R. A., Gianninas A., Ruiz M. T., Limoges M.-M., Dufour P., Fontaine G., Liebert J., A Comprehensive Spectroscopic Analysis of DB White Dwarfs, ApJ, 2011, vol. 737, p. 28

Bernstein R., Shectman S. A., Gunnels S. M., Mochnacki S., Athey A. E., MIKE: A Double Echelle Spectrograph for the Magellan Telescopes at Las Campanas Observatory. In Instrument Design and Performance for Optical/Infrared Ground-based Telescopes, vol. 4841 of Society of Photo-Optical Instrumentation Engineers (SPIE) Conference Series, 2003, p. 1694

Biazzo K., Gratton R., Desidera S., et al, The GAPS programme with HARPS-N at TNG. X. Differential abundances in the XO-2 planet-hosting binary, A\&A, 2015, vol. 583, p. A135

Boggs P. T., Rogers J. E., Orthogonal distance regression, Contemporary Mathematics, 1990, vol. 112 , p. 183

Bolmont E., Raymond S. N., Leconte J., Matt S. P., Effect of the stellar spin history on the tidal evolution of close-in planets, A\&A, 2012, vol. 544, p. A124

Bolton A. S., Schlegel D. J., Spectro-Perfectionism: An Algorithmic Framework for Photon Noise-Limited Extraction of Optical Fiber Spectroscopy, PASP, 2010, vol. 122, p. 248

Borucki W., Koch D., Batalha N., Caldwell D., Christensen-Dalsgaard J., Cochran W. D., Dunham E., Gautier T. N., Geary J., Gilliland R., Jenkins J., Kjeldsen H., Lissauer J. J., Rowe J., KEPLER: Search for Earth-Size Planets in the Habitable Zone. In Transiting Planets, vol. 253 of IAU Symposium, 2009, p. 289

Brown D. J. A., Discrepancies between isochrone fitting and gyrochronology for exoplanet host stars?, MNRAS, 2014, vol. 442, p. 1844

Butler R. P., Vogt S. S., Laughlin G., Burt J. A., Rivera E. J., Tuomi M., Teske J., Arriagada P., Diaz M., Holden B., Keiser S., The LCES HIRES/Keck Precision Radial Velocity Exoplanet Survey, AJ, 2017, vol. 153, p. 208

Carlos M., Nissen P. E., Meléndez J., Correlation between lithium abundances and ages of solar twin stars, A\&A, 2016, vol. 587, p. A100

Castelli F., Kurucz R. L., New Grids of ATLAS9 Model Atmospheres, ArXiv Astrophysics eprints, 2004 
Ceillier T., García R. A., Salabert D., Mathur S., Extracting surface rotation periods of solarlike Kepler targets. In European Physical Journal Web of Conferences , vol. 101 of European Physical Journal Web of Conferences, 2015, p. 06016

Ceillier T., van Saders J., García R. A., Metcalfe T. S., Creevey O., Mathis S., Mathur S., Pinsonneault M. H., Salabert D., Tayar J., Rotation periods and seismic ages of KOIs - comparison with stars without detected planets from Kepler observations, MNRAS, 2016, vol. 456, p. 119

Chanamé J., Ramírez I., Toward Precise Ages for Single Stars in the Field. Gyrochronology Constraints at Several Gyr Using Wide Binaries. I. Ages for Initial Sample, ApJ, 2012, vol. 746, p. 102

Chaplin W. J., Basu S., et al., Asteroseismic Fundamental Properties of Solar-type Stars Observed by the NASA Kepler Mission, ApJS, 2014, vol. 210, p. 1

Charbonneau P., Modeling Stellar Angular Momentum Evolution (Invited Review). In Cool Stars, Stellar Systems, and the Sun, vol. 26 of Astronomical Society of the Pacific Conference Series, 1992, p. 416

Charbonneau P., Solar Dynamo Theory, ARA\&A, 2014, vol. 52, p. 251

Charbonnel C., Vauclair S., Maeder A., Meynet G., Schaller G., Lithium and beryllium depletion by rotation-induced mixing in the Sun and in galactic cluster stars, A\&A, 1994, vol. 283, p. 155

Crepp J. R., Johnson J. A., Howard A. W., Marcy G. W., Brewer J., Fischer D. A., Wright J. T., Isaacson H., The TRENDS High-contrast Imaging Survey. V. Discovery of an Old and Cold Benchmark T-dwarf Orbiting the Nearby G-star HD 19467, ApJ, 2014, vol. 781, p. 29

Crepp J. R., Johnson J. A., Howard A. W., Marcy G. W., Gianninas A., Kilic M., Wright J. T., The TRENDS High-contrast Imaging Survey. III. A Faint White Dwarf Companion Orbiting HD 114174, ApJ, 2013, vol. 774, p. 1

Crepp J. R., Rice E. L., Veicht A., et al., Direct Spectrum of the Benchmark T Dwarf HD 19467 B, ApJ, 2015, vol. 798, p. L43

Cuntz M., Saar S. H., Musielak Z. E., On Stellar Activity Enhancement Due to Interactions with Extrasolar Giant Planets, ApJ, 2000, vol. 533, p. L151 
Czekala I., Mandel K. S., Andrews S. M., Dittmann J. A., Ghosh S. K., Montet B. T., Newton E. R., Disentangling Time-series Spectra with Gaussian Processes: Applications to Radial Velocity Analysis, ApJ, 2017, vol. 840, p. 49

Desidera S., D’Orazi V., Lugaro M., Is beryllium ultra-depletion in solar-type stars linked to the presence of a white dwarf companion?, A\&A, 2016, vol. 587, p. A46

do Nascimento J.-D., da Costa J. S., Castro M., Rotational periods and evolutionary models for subgiant stars observed by CoRoT, A\&A, 2012, vol. 548, p. L1

do Nascimento Jr. J. D., Castro M., Meléndez J., Bazot M., Théado S., Porto de Mello G. F., de Medeiros J. R., Age and mass of solar twins constrained by lithium abundance, A\&A, 2009, vol. 501, p. 687

do Nascimento Jr. J.-D., García R. A., Mathur S., Anthony F., Barnes S. A., Meibom S., da Costa J. S., Castro M., Salabert D., Ceillier T., Rotation Periods and Ages of Solar Analogs and Solar Twins Revealed by the Kepler Mission, ApJ, 2014, vol. 790, p. L23

do Nascimento Jr. J.-D., Vidotto A. A., Petit P., Folsom C., Castro M., Marsden S. C., Morin J., Porto de Mello G. F., Meibom S., Jeffers S. V., Guinan E., Ribas I., Magnetic Field and Wind of Kappa Ceti: Toward the Planetary Habitability of the Young Sun When Life Arose on Earth, ApJ, 2016, vol. 820, p. L15

Dong C., Jin M., Lingam M., Airapetian V. S., Ma Y., van der Holst B., Atmospheric escape from the TRAPPIST-1 planets and implications for habitability, ArXiv e-prints, 2017

dos Santos L. A., Meléndez J., Bedell M., Bean J. L., Spina L., Alves-Brito A., Dreizler S., Ramírez I., Asplund M., Spectroscopic binaries in the Solar Twin Planet Search program: from substellar-mass to $\mathrm{M}$ dwarf companions, 2017, in press

dos Santos L. A., Meléndez J., do Nascimento J.-D., Bedell M., Ramírez I., Bean J. L., Asplund M., Spina L., Dreizler S., Alves-Brito A., Casagrande L., The Solar Twin Planet Search. IV. The Sun as a typical rotator and evidence for a new rotational braking law for Sun-like stars, A\&A, 2016, vol. 592, p. A156

Dotter A., Conroy C., Cargile P., Asplund M., The Influence of Atomic Diffusion on Stellar Ages and Chemical Tagging, ApJ, 2017, vol. 840, p. 99 
Doyle A. P., Davies G. R., Smalley B., Chaplin W. J., Elsworth Y., Determining stellar macroturbulence using asteroseismic rotational velocities from Kepler, MNRAS, 2014, vol. 444, p. 3592

Duquennoy A., Mayor M., Multiplicity among solar-type stars in the solar neighbourhood. II Distribution of the orbital elements in an unbiased sample, A\&A, 1991, vol. 248, p. 485

Eastman J., Gaudi B. S., Agol E., EXOFAST: A Fast Exoplanetary Fitting Suite in IDL, PASP, 2013 , vol. 125 , p. 83

Egeland R., Soon W., Baliunas S., Hall J. C., Pevtsov A. A., Bertello L., The Mount Wilson Observatory S-index of the Sun, ApJ, 2017, vol. 835, p. 25

Feng Y. K., Wright J. T., Nelson B., Wang S. X., Ford E. B., Marcy G. W., Isaacson H., Howard A. W., The California Planet Survey IV: A Planet Orbiting the Giant Star HD 145934 and Updates to Seven Systems with Long-period Planets, ApJ, 2015, vol. 800, p. 22

Ferraz-Mello S., Tadeu dos Santos M., Folonier H., Czismadia S., do Nascimento Jr. J.-D., Pätzold M., Interplay of Tidal Evolution and Stellar Wind Braking in the Rotation of Stars Hosting Massive Close-In Planets, ApJ, 2015, vol. 807, p. 78

Figueira P., Santerne A., Suárez Mascareño A., Gomes da Silva J., et al. Is the activity level of HD 80606 influenced by its eccentric planet?, A\&A, 2016, vol. 592, p. A143

Fischer D. A., Marcy G. W., Spronck J. F. P., The Twenty-five Year Lick Planet Search, ApJS, 2014, vol. 210, p. 5

Foreman-Mackey D., Hogg D. W., Lang D., Goodman J., emcee: The MCMC Hammer, PASP, 2013, vol. 125 , p. 306

Freitas F. C., Usando gêmeas solares para estudar a conexão entre atividade estelar e idade, Universidade de São Paulo, 2017, Master Dissertation

Freitas F. C., Meléndez J., Bedell M., Ramírez I., Bean J. L., Asplund M., Spina L., Dreizler S., Alves-Brito A., Casagrande L., The Solar Twin Planet Search. VI. The age-chromospheric activity relation, 2017, submitted

Fuhrmann K., Chini R., Kaderhandt L., Chen Z., Multiplicity among Solar-type Stars, ApJ, 2017, vol. 836, p. 139 
Gallet F., Bouvier J., Improved angular momentum evolution model for solar-like stars, A\&A, 2013, vol. 556, p. A36

García R. A., Ceillier T., Salabert D., et al., Rotation and magnetism of Kepler pulsating solarlike stars. Towards asteroseismically calibrated age-rotation relations, A\&A, 2014, vol. 572, p. A34

Gray D. F., Atmospheric turbulence measured in stars above the main sequence, ApJ, 1975, vol. 202, p. 148

Gray D. F., Measurements of rotation and turbulence in F, G, and K dwarfs, ApJ, 1984, vol. 281, p. 719

Gray D. F., The Observation and Analysis of Stellar Photospheres, 2005

Grether D., Lineweaver C. H., How Dry is the Brown Dwarf Desert? Quantifying the Relative Number of Planets, Brown Dwarfs, and Stellar Companions around Nearby Sun-like Stars, ApJ, 2006, vol. 640, p. 1051

Guinan E. F., Engle S. G., The Sun in time: age, rotation, and magnetic activity of the Sun and solar-type stars and effects on hosted planets. In The Ages of Stars, vol. 258 of IAU Symposium, 2009, p. 395

Gustafsson B., Is the Sun a Sun-Like Star?, Space Sci. Rev., 1998, vol. 85, p. 419

Gustafsson B., Is the Sun unique as a star-and if so, why?, Physica Scripta Volume T, 2008, vol. 130, p. 014036

Hartkopf W. I., Tokovinin A., Mason B. D., Speckle Interferometry at SOAR in 2010 and 2011: Measures, Orbits, and Rectilinear Fits, AJ, 2012, vol. 143, p. 42

Haywood R. D., Hide and Seek: Radial-Velocity Searches for Planets around Active Stars, University of St Andrews, 2015, Ph.D. Thesis

Haywood R. D., Collier Cameron A., Unruh Y. C., Lovis C., Lanza A. F., Llama J., Deleuil M., Fares R., Gillon M., Moutou C., Pepe F., Pollacco D., Queloz D., Ségransan D., The Sun as a planet-host star: proxies from SDO images for HARPS radial-velocity variations, MNRAS, 2016, vol. 457, p. 3637 
Hekker S., Meléndez J., Precise radial velocities of giant stars. III. Spectroscopic stellar parameters, A\&A, 2007, vol. 475, p. 1003

Horne K., An optimal extraction algorithm for CCD spectroscopy, PASP, 1986, vol. 98, p. 609

Howard R., Harvey J., Spectroscopic Determinations of Solar Rotation, Sol. Phys., 1970, vol. 12, p. 23

Jeffries R. D., Using rotation, magnetic activity and lithium to estimate the ages of low mass stars. In EAS Publications Series, vol. 65 of EAS Publications Series, 2014, p. 289

Jenkins J. S., Díaz M., Jones H. R. A., Butler R. P., Tinney C. G., O’Toole S. J., Carter B. D., Wittenmyer R. A., Pinfield D. J., The observed distribution of spectroscopic binaries from the Anglo-Australian Planet Search, MNRAS, 2015, vol. 453, p. 1439

Jurgenson C., Fischer D., McCracken T., Sawyer D., Szymkowiak A., Davis A., Muller G., Santoro F., EXPRES: a next generation RV spectrograph in the search for earth-like worlds. In Ground-based and Airborne Instrumentation for Astronomy VI, vol. 9908 of Proc. SPIE, 2016, p. $99086 \mathrm{~T}$

Kane S. R., Hill M. L., Kasting J. F., Kopparapu R. K., Quintana E. V., Barclay T., Batalha N. M., Borucki W. J., Ciardi D. R., Haghighipour N., Hinkel N. R., Kaltenegger L., Selsis F., Torres G., A Catalog of Kepler Habitable Zone Exoplanet Candidates, ApJ, 2016, vol. 830, p. 1

Karoff C., Albrecht S., Bonanno A., Faurschou Knudsen M., Chromosomes Emission of Planet Candidate Host Stars: A Way to Identify False Positives, ApJ, 2016, vol. 830, p. L7

Kawaler S. D., Angular momentum loss in low-mass stars, ApJ, 1988, vol. 333, p. 236

Kiseleva-Eggleton L., Eggleton P. P., Not-So-Simple Triple Systems. In Evolution of Binary and Multiple Star Systems, vol. 229 of Astronomical Society of the Pacific Conference Series, 2001, p. 91

Kjurkchieva D., Vasileva D., Dimitrov D., Light Curve Solutions of 12 Eccentric Kepler Binaries and Analysis of Their Out-of-eclipse Variability, AJ, 2016, vol. 152, p. 189

Kraft R. P., Studies of Stellar Rotation. V. The Dependence of Rotation on Age among SolarType Stars, ApJ, 1967, vol. 150, p. 551 
Lachaume R., Dominik C., Lanz T., Habing H. J., Age determinations of main-sequence stars: combining different methods, A\&A, 1999, vol. 348, p. 897

Lanzafame A. C., Spada F., Rotational evolution of slow-rotator sequence stars, A\&A, 2015, vol. 584, p. A30

Latham D. W., Stefanik R. P., Torres G., Davis R. J., Mazeh T., Carney B. W., Laird J. B., Morse J. A., A Survey of Proper-Motion Stars. XVI. Orbital Solutions for 171 Single-lined Spectroscopic Binaries, AJ, 2002, vol. 124, p. 1144

Leão I. C., Pasquini L., Ferreira Lopes C. E., Neves V., Valcarce A. A. R., de Oliveira L. L. A., Freire da Silva D., de Freitas D. B., Canto Martins B. L., Janot-Pacheco E., Baglin A., De Medeiros J. R., Rotation period distribution of CoRoT and Kepler Sun-like stars, A\&A, 2015, vol. 582 , p. A85

Lebreton Y., Montalbán J., Stellar ages from asteroseismology. In The Ages of Stars, vol. 258 of IAU Symposium, 2009, p. 419

Li T. D., Bi S. L., Liu K., Tian Z. J., Shuai G. Z., Stellar parameters and seismological analysis of the star 18 Scorpii, A\&A, 2012, vol. 546, p. A83

Lorenzo-Oliveira D., Porto de Mello G. F., Schiavon R. P., The age-mass-metallicity-activity relation for solar-type stars: comparisons with asteroseismology and the NGC 188 open cluster, A\&A, 2016, vol. 594, p. L3

Lovis C., Dumusque X., Santos N. C., Bouchy F., Mayor M., Pepe F., Queloz D., Ségransan D., Udry S., The HARPS search for southern extra-solar planets. XXXI. Magnetic activity cycles in solar-type stars: statistics and impact on precise radial velocities, ArXiv e-prints, 2011

Luhman K. L., A Search for a Distant Companion to the Sun with the Wide-field Infrared Survey Explorer, ApJ, 2014, vol. 781, p. 4

Magic Z., Collet R., Asplund M., Trampedach R., Hayek W., Chiavassa A., Stein R. F., Nordlund $\AA$., The Stagger-grid: A grid of 3D stellar atmosphere models. I. Methods and general properties, A\&A, 2013, vol. 557, p. A26

Makarov V. V., Kaplan G. H., Statistical Constraints for Astrometric Binaries with Nonlinear Motion, AJ, 2005, vol. 129, p. 2420 
Maldonado J., Martínez-Arnáiz R. M., Eiroa C., Montes D., Montesinos B., A spectroscopy study of nearby late-type stars, possible members of stellar kinematic groups, A\&A, 2010, vol. 521, p. A12

Mamajek E. E., Hillenbrand L. A., Improved Age Estimation for Solar-Type Dwarfs Using Activity-Rotation Diagnostics, ApJ, 2008, vol. 687, p. 1264

Marsden S. C., Petit P., Jeffers S. V., et al. A BCool magnetic snapshot survey of solar-type stars, MNRAS, 2014, vol. 444, p. 3517

Mason B. D., Hartkopf W. I., Holdenried E. R., Rafferty T. J., Speckle Interferometry of New and Problem Hipparcos Binaries. II. Observations Obtained in 1998-1999 from McDonald Observatory, AJ, 2001, vol. 121, p. 3224

Matthews C. T., Crepp J. R., Skemer A., Hinz P. M., Gianninas A., Kilic M., Skrutskie M., Bailey V. P., Defrere D., Leisenring J., Esposito S., Puglisi A., Mid-infrared High-contrast Imaging of HD 114174 B: An Apparent Age Discrepancy in a "Sirius-like" Binary System, ApJ, 2014, vol. 783 , p. L25

Mayor M., Pepe F., Queloz D., et al., Setting New Standards with HARPS, The Messenger, 2003, vol. 114, p. 20

Mayor M., Queloz D., A Jupiter-mass companion to a solar-type star, Nature, 1995, vol. 378, p. 355

McQuillan A., Mazeh T., Aigrain S., Stellar Rotation Periods of the Kepler Objects of Interest: A Dearth of Close-in Planets around Fast Rotators, ApJ, 2013, vol. 775, p. L11

Meibom S., Barnes S. A., Latham D. W., et al. The Kepler Cluster Study: Stellar Rotation in NGC 6811, ApJ, 2011, vol. 733, p. L9

Meibom S., Barnes S. A., Platais I., Gilliland R. L., Latham D. W., Mathieu R. D., A spin-down clock for cool stars from observations of a 2.5-billion-year-old cluster, Nature, 2015, vol. 517, p. 589

Meléndez J., Asplund M., Gustafsson B., Yong D., The Peculiar Solar Composition and Its Possible Relation to Planet Formation, ApJ, 2009, vol. 704, p. L66 
Meléndez J., Bean J. L., Bedell M., Ramírez I., Asplund M., Dreizler S., Alves-Brito A., Spina L., Casagrande L., Monroe T., Maia M. T., Freitas F., Using Solar Twins to Explore the PlanetStar Connection with Unparallelled Precision, The Messenger, 2015, vol. 161, p. 28

Meléndez J., Bedell M., Bean J. L., Ramírez I., Asplund M., Dreizler S., Yan H.-L., Shi J.-R., Lind K., Ferraz-Mello S., Galarza J. Y., dos Santos L., Spina L., Maia M. T., Alves-Brito A., Monroe T., Casagrande L., The Solar Twin Planet Search. V. Close-in, low-mass planet candidates and evidence of planet accretion in the solar twin HIP 68468, A\&A, 2017, vol. 597, p. A34

Meléndez J., Bergemann M., Cohen J. G., Endl M., Karakas A. I., Ramírez I., Cochran W. D., Yong D., MacQueen P. J., Kobayashi C., Asplund M., The remarkable solar twin HIP 56948: a prime target in the quest for other Earths, A\&A, 2012, vol. 543, p. A29

Melo C. H. F., Pasquini L., De Medeiros J. R., Accurate Vsin i measurements in M 67: The angular momentum evolution of $1.2 \mathrm{M}_{\text {sun }}$ stars, A\&A, 2001, vol. 375, p. 851

Mengel M. W., Marsden S. C., Carter B. D., Horner J., King R., Fares R., Jeffers S. V., Petit P., Vidotto A. A., Morin J., BCool Collaboration A BCool survey of the magnetic fields of planet-hosting solar-type stars, MNRAS, 2017, vol. 465, p. 2734

Mestel L., The interaction of rotation and magnetic field in the solar system, Philosophical Transactions of the Royal Society of London Series A, 1984, vol. 313, p. 19

Morton T. D., Bryson S. T., Coughlin J. L., Rowe J. F., Ravichandran G., Petigura E. A., Haas M. R., Batalha N. M., False Positive Probabilities for all Kepler Objects of Interest: 1284 Newly Validated Planets and 428 Likely False Positives, ApJ, 2016, vol. 822, p. 86

Moultaka J., Ilovaisky S. A., Prugniel P., Soubiran C., The ELODIE Archive, PASP, 2004, vol. 116, p. 693

Murray C. D., Correia A. C. M., , 2010 Keplerian Orbits and Dynamics of Exoplanets. pp 15-23

Newville M., Stensitzki T., Allen D. B., Rawlik M., Ingargiola A., Nelson A., , 2016 Lmfit: Non-Linear Least-Square Minimization and Curve-Fitting for Python Astrophysics Source Code Library 
Nissen P. E., High-precision abundances of elements in solar twin stars. Trends with stellar age and elemental condensation temperature, A\&A, 2015, vol. 579, p. A52

Noyes R. W., Hartmann L. W., Baliunas S. L., Duncan D. K., Vaughan A. H., Rotation, convection, and magnetic activity in lower main-sequence stars, ApJ, 1984, vol. 279, p. 763

Noyes R. W., Weiss N. O., Vaughan A. H., The relation between stellar rotation rate and activity cycle periods, ApJ, 1984, vol. 287, p. 769

Pace G., Pasquini L., The age-activity-rotation relationship in solar-type stars, A\&A, 2004, vol. 426 , p. 1021

Parker E. N., Dynamics of the Interplanetary Gas and Magnetic Fields., ApJ, 1958, vol. 128, p. 664

Paxton B., Bildsten L., Dotter A., Herwig F., Lesaffre P., Timmes F., Modules for Experiments in Stellar Astrophysics (MESA), ApJS, 2011, vol. 192, p. 3

Paxton B., Marchant P., Schwab J., Bauer E. B., Bildsten L., Cantiello M., Dessart L., Farmer R., Hu H., Langer N., Townsend R. H. D., Townsley D. M., Timmes F. X., Modules for Experiments in Stellar Astrophysics (MESA): Binaries, Pulsations, and Explosions, ApJS, 2015, vol. 220, p. 15

Perruchot S., Bouchy F., Chazelas B., Díaz R. F., Hébrard G., Arnaud K., Arnold L., Avila G., Delfosse X., Boisse I., Moreaux G., Pepe F., Richaud Y., Santerne A., Sottile R., Tézier D., Higher-precision radial velocity measurements with the SOPHIE spectrograph using octagonal-section fibers. In Techniques and Instrumentation for Detection of Exoplanets V , vol. 8151 of Proc. SPIE, 2011, p. 815115

Pinsonneault M. H., Kawaler S. D., Sofia S., Demarque P., Evolutionary models of the rotating sun, ApJ, 1989, vol. 338, p. 424

Piskunov N. E., Valenti J. A., New algorithms for reducing cross-dispersed echelle spectra, A\&A, 2002, vol. 385, p. 1095

Pizzolato N., Maggio A., Micela G., Sciortino S., Ventura P., The stellar activity-rotation relationship revisited: Dependence of saturated and non-saturated X-ray emission regimes on stellar mass for late-type dwarfs, A\&A, 2003, vol. 397, p. 147 
Queloz D., Echelle Spectroscopy with a CCD at Low Signal-To-Noise Ratio. In New Developments in Array Technology and Applications , vol. 167 of IAU Symposium, 1995, p. 221

Queloz D., Henry G. W., Sivan J. P., Baliunas S. L., Beuzit J. L., Donahue R. A., Mayor M., Naef D., Perrier C., Udry S., No planet for HD 166435, A\&A, 2001, vol. 379, p. 279

Raghavan D., McAlister H. A., Henry T. J., Latham D. W., Marcy G. W., Mason B. D., Gies D. R., White R. J., ten Brummelaar T. A., A Survey of Stellar Families: Multiplicity of Solartype Stars, ApJS, 2010, vol. 190, p. 1

Ramírez I., Allende Prieto C., Lambert D. L., Oxygen Abundances in Nearby FGK Stars and the Galactic Chemical Evolution of the Local Disk and Halo, ApJ, 2013, vol. 764, p. 78

Ramírez I., Meléndez J., Bean J., Asplund M., Bedell M., Monroe T., Casagrande L., Schirbel L., Dreizler S., Teske J., Tucci Maia M., Alves-Brito A., Baumann P., The Solar Twin Planet Search. I. Fundamental parameters of the stellar sample, A\&A, 2014, vol. 572, p. A48

Rhodes Jr. E. J., Kosovichev A. G., Schou J., Scherrer P. H., Reiter J., Measurements of Frequencies of Solar Oscillations from the MDI Medium-1 Program, Sol. Phys., 1997, vol. 175, p. 287

Ribas I., Guinan E. F., Güdel M., Audard M., Evolution of the Solar Activity over Time and Effects on Planetary Atmospheres. I. High-Energy Irradiances (1-1700 Å), ApJ, 2005, vol. 622, p. 680

Riddle R. L., Tokovinin A., Mason B. D., Hartkopf W. I., Roberts Jr. L. C., Baranec C., Law N. M., Bui K., Burse M. P., Das H. K., Dekany R. G., Kulkarni S., Punnadi S., Ramaprakash A. N., Tendulkar S. P., A Survey of the High Order Multiplicity of Nearby Solar-type Binary Stars with Robo-AO, ApJ, 2015, vol. 799, p. 4

Robles J. A., Lineweaver C. H., Grether D., Flynn C., Egan C. A., Pracy M. B., Holmberg J., Gardner E., A Comprehensive Comparison of the Sun to Other Stars: Searching for SelfSelection Effects, ApJ, 2008, vol. 684, p. 691

Rogers L. A., Most 1.6 Earth-radius Planets are Not Rocky, ApJ, 2015, vol. 801, p. 41

Saffe C., Flores M., Buccino A., HD 80606: searching for the chemical signature of planet formation, A\&A, 2015, vol. 582, p. A17 
Schatzman E., A theory of the role of magnetic activity during star formation, Annales d'Astrophysique, 1962, vol. 25, p. 18

Schirbel L., Meléndez J., Karakas A. I., Ramírez I., Castro M., Faria M. A., Lugaro M., Asplund M., Tucci Maia M., Yong D., Howes L., do Nascimento J. D., HIP 10725: The first solar twin/analogue field blue straggler, A\&A, 2015, vol. 584, p. A116

Seifahrt A., Bean J. L., Stürmer J., Gers L., Grobler D. S., Reed T., Jones D. J., Development and construction of MAROON-X. In Ground-based and Airborne Instrumentation for Astronomy VI , vol. 9908 of Proc. SPIE, 2016, p. 990818

Shields A. L., Ballard S., Johnson J. A., The Habitability of Planets Orbiting M-dwarf Stars, ArXiv e-prints, 2016

Silva Aguirre V., Davies G. R., Basu S., Christensen-Dalsgaard J., Creevey O., Metcalfe T. S., Bedding T. R., et al. Ages and fundamental properties of Kepler exoplanet host stars from asteroseismology, MNRAS, 2015, vol. 452, p. 2127

Skumanich A., Time Scales for CA II Emission Decay, Rotational Braking, and Lithium Depletion, ApJ, 1972, vol. 171, p. 565

Smith M. A., Rotational studies of lower main-sequence stars, PASP, 1979, vol. 91, p. 737

Sneden C. A., Carbon and Nitrogen Abundances in Metal-Poor Stars, The University of Texas at Austin, 1973, Ph.D. Thesis

Soderblom D. R., Rotational studies of late-type stars. II - Ages of solar-type stars and the rotational history of the sun, ApJS, 1983, vol. 53, p. 1

Soderblom D. R., A survey of chromospheric emission and rotation among solar-type stars in the solar neighborhood, AJ, 1985, vol. 90, p. 2103

Soderblom D. R., The Ages of Stars, ARA\&A, 2010, vol. 48, p. 581

Soderblom D. R., Duncan D. K., Johnson D. R. H., The chromospheric emission-age relation for stars of the lower main sequence and its implications for the star formation rate, ApJ, 1991, vol. 375 , p. 722 
Soderblom D. R., Stauffer J. R., Hudon J. D., Jones B. F., Rotation and chromospheric emission among F, G, and K dwarfs of the Pleiades, ApJS, 1993, vol. 85, p. 315

Sood A., Kim E.-j., Hollerbach R., Dynamical Model for Spindown of Solar-type Stars, ApJ, 2016, vol. 832, p. 97

Spada F., Demarque P., Kim Y.-C., Boyajian T. S., Brewer J. M., The Yale-Potsdam Stellar Isochrones, ApJ, 2017, vol. 838, p. 161

Stancliffe R. J., Fossati L., Passy J.-C., Schneider F. R. N., Confronting uncertainties in stellar physics. II. Exploring differences in main-sequence stellar evolution tracks, A\&A, 2016, vol. 586, p. A119

Steffen J. H., Coughlin J. L., A Population of planetary systems characterized by short-period, Earth-sized planets, Proceedings of the National Academy of Science, 2016, vol. 113, p. 12023

Strassmeier K. G., Weber M., Granzer T., Järvinen S., Rotation, activity, and lithium abundance in cool binary stars, Astronomische Nachrichten, 2012, vol. 333, p. 663

Szentgyorgyi A., Frebel A., Furesz G., et al. The GMT-CfA, Carnegie, Catolica, Chicago Large Earth Finder (G-CLEF): a general purpose optical echelle spectrograph for the GMT with precision radial velocity capability. In Ground-based and Airborne Instrumentation for Astronomy IV , vol. 8446 of Proc. SPIE, 2012, p. 84461H

Takeda Y., Tajitsu A., Honda S., Kawanomoto S., Ando H., Sakurai T., Beryllium Abundances of Solar-Analog Stars, PASJ, 2011, vol. 63, p. 697

Talon S., Zahn J.-P., Anisotropic diffusion and shear instabilities., A\&A, 1997, vol. 317, p. 749

Tokovinin A., From Binaries to Multiples. I. Data on F and G Dwarfs within 67 pc of the Sun, AJ, 2014a, vol. 147, p. 86

Tokovinin A., From Binaries to Multiples. II. Hierarchical Multiplicity of F and G Dwarfs, AJ, 2014b, vol. 147 , p. 87

Torres G., Substellar Companion Masses from Minimal Radial Velocity or Astrometric Information: a Monte Carlo Approach, PASP, 1999, vol. 111, p. 169 
Tucci Maia M., Meléndez J., Castro M., Asplund M., Ramírez I., Monroe T. R., do Nascimento Jr. J. D., Yong D., Shallow extra mixing in solar twins inferred from Be abundances, A\&A, 2015, vol. 576, p. L10

Tucci Maia M., Ramírez I., Meléndez J., Bedell M., Bean J. L., Asplund M., The Solar Twin Planet Search. III. The [Y/Mg] clock: estimating stellar ages of solar-type stars, A\&A, 2016, vol. 590, p. A32

van Leeuwen F., Validation of the new Hipparcos reduction, A\&A, 2007, vol. 474, p. 653

van Saders J. L., Ceillier T., Metcalfe T. S., Aguirre V. S., Pinsonneault M. H., García R. A., Mathur S., Davies G. R., Weakened magnetic braking as the origin of anomalously rapid rotation in old field stars, Nature, 2016, vol. 529, p. 181

Vidotto A. A., Gregory S. G., Jardine M., Donati J. F., Petit P., Morin J., Folsom C. P., Bouvier J., Cameron A. C., Hussain G., Marsden S., Waite I. A., Fares R., Jeffers S., do Nascimento J. D., Stellar magnetism: empirical trends with age and rotation, MNRAS, 2014, vol. 441, p. 2361

Vogel S. N., Kuhi L. V., Rotational velocities of pre-main-sequence stars, ApJ, 1981, vol. 245, p. 960

Vogt S. S., Allen S. L., Bigelow B. C., et al., HIRES: the high-resolution echelle spectrometer on the Keck 10-m Telescope. In Instrumentation in Astronomy VIII , vol. 2198 of Proc. SPIE, 1994, p. 362

Willmarth D. W., Fekel F. C., Abt H. A., Pourbaix D., Spectroscopic Orbits for 15 Late-type Stars, AJ, 2016, vol. 152, p. 46

Wilson O. C., Chromospheric variations in main-sequence stars, ApJ, 1978, vol. 226, p. 379

Wilson P. A., Hébrard G., Santos N. C., et al. The SOPHIE search for northern extrasolar planets. IX. Populating the brown dwarf desert, A\&A, 2016, vol. 588, p. A144

Yana Galarza J., Análise da composição química das gêmeas solares HIP 100963, HD 45184 e a descoberta da gêmea solar Inti 1, Universidade de São Paulo, 2016, Master Dissertation

Yana Galarza J., Meléndez J., Ramírez I., Yong D., Karakas A. I., Asplund M., Liu F., Highprecision analysis of the solar twin HIP 100963, A\&A, 2016, vol. 589, p. A17 
Yi S., Demarque P., Kim Y.-C., Lee Y.-W., Ree C. H., Lejeune T., Barnes S., Toward Better Age Estimates for Stellar Populations: The $\mathrm{Y}^{2}$ Isochrones for Solar Mixture, ApJS, 2001, vol. 136, p. 417

Zahn J.-P., Circulation and turbulence in rotating stars, A\&A, 1992, vol. 265, p. 115

Zechmeister M., Anglada-Escudé G., Reiners A., Flat-relative optimal extraction. A quick and efficient algorithm for stabilised spectrographs, A\&A, 2014, vol. 561, p. A59 
Appendix 



\section{Appendix A}

\section{The stellar parameters of the solar twins}

The following stellar parameters of the solar twins in our program were obtained with the MIKE spectra following the methods in Ramírez et al. (2014) and Tucci Maia et al. (2015). More details about the MIKE data are available in Chapter 2 and Section 3.3. The original version of Table A.1 is also available in electronic form at the CDS via anonymous ftp to cdsarc.u-strasbg.fr (130.79.128.5) or via http://cdsweb.u-strasbg.fr/cgi-bin/qcat? J/A+A/592/A156. 


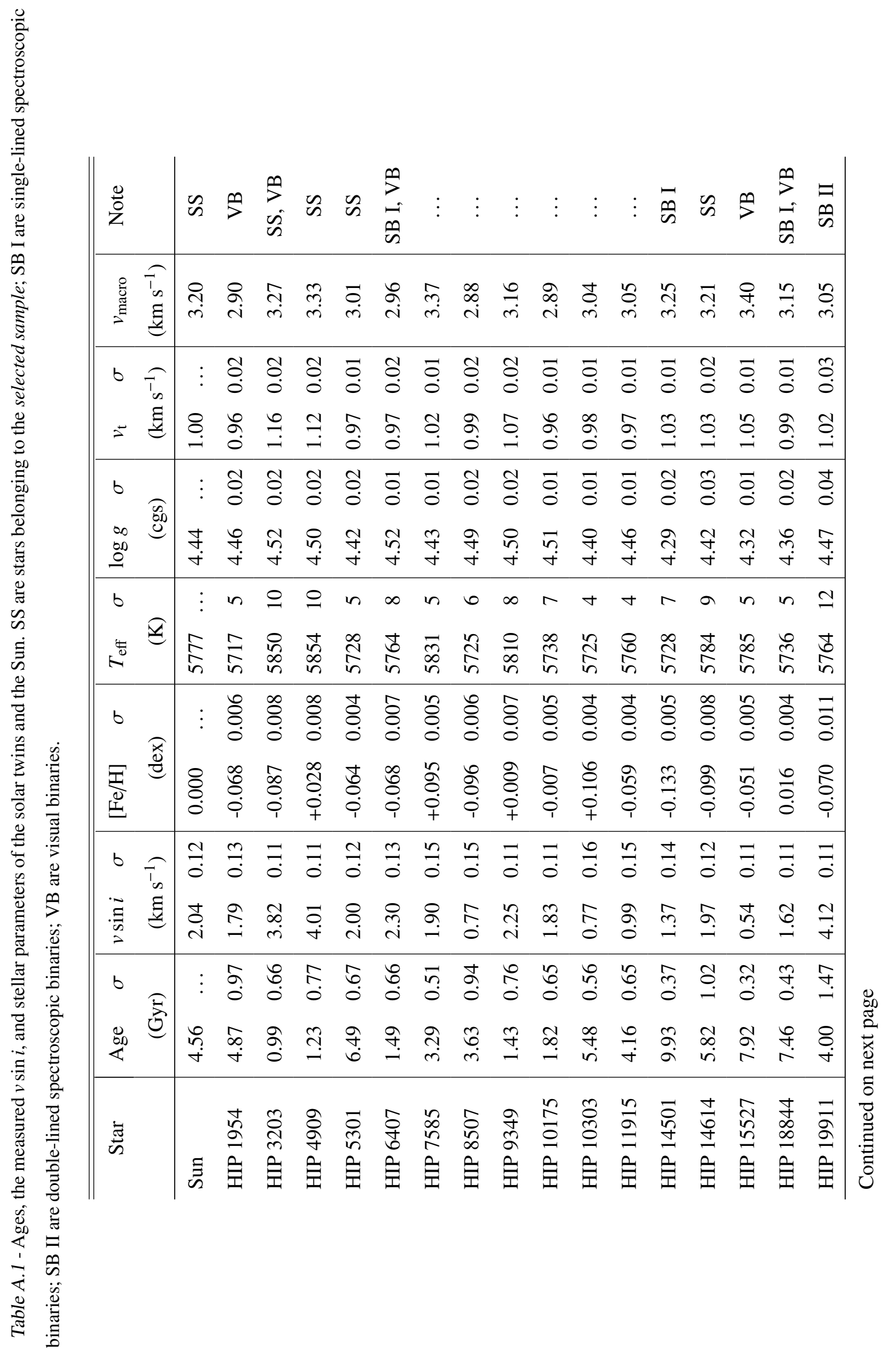




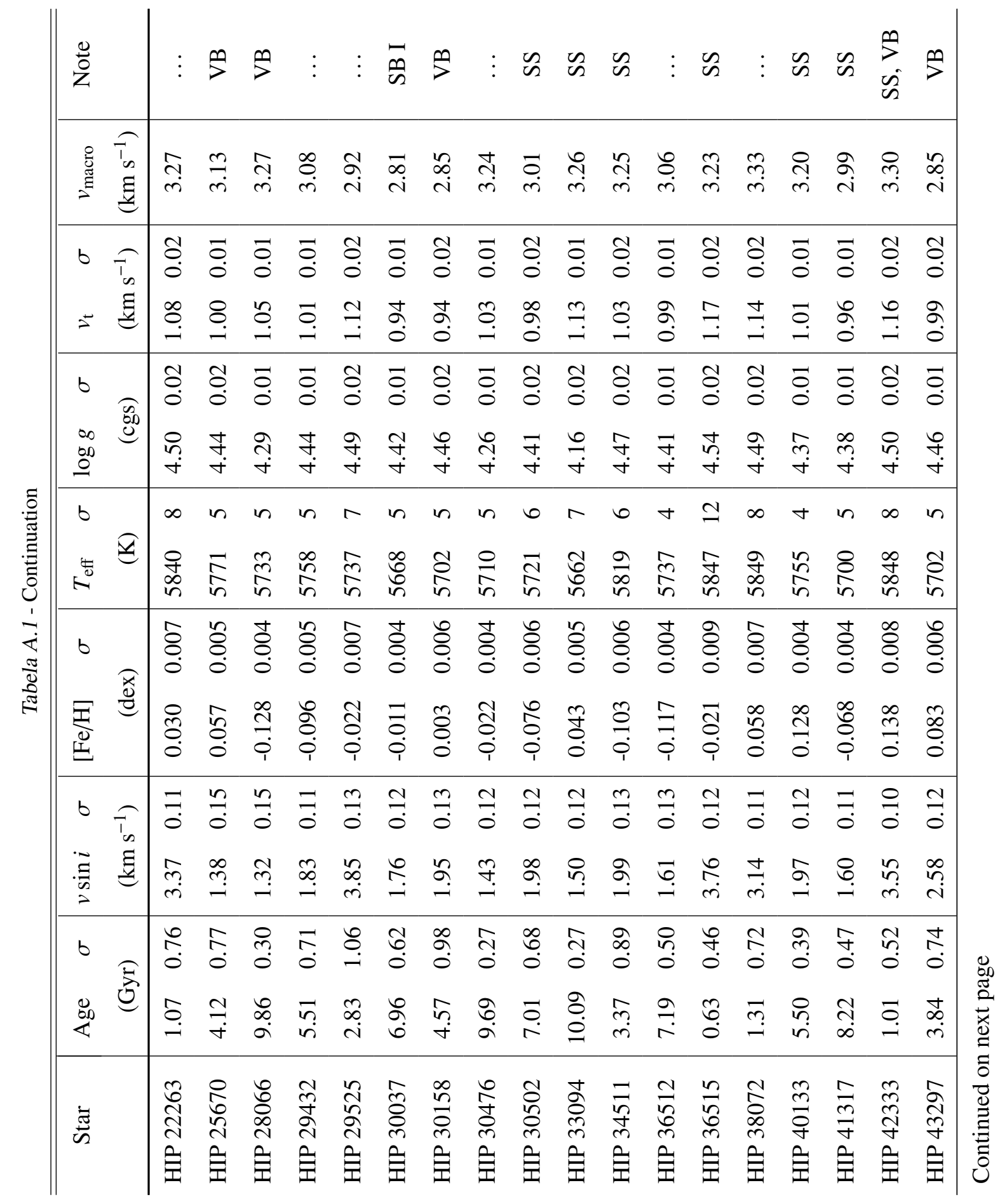




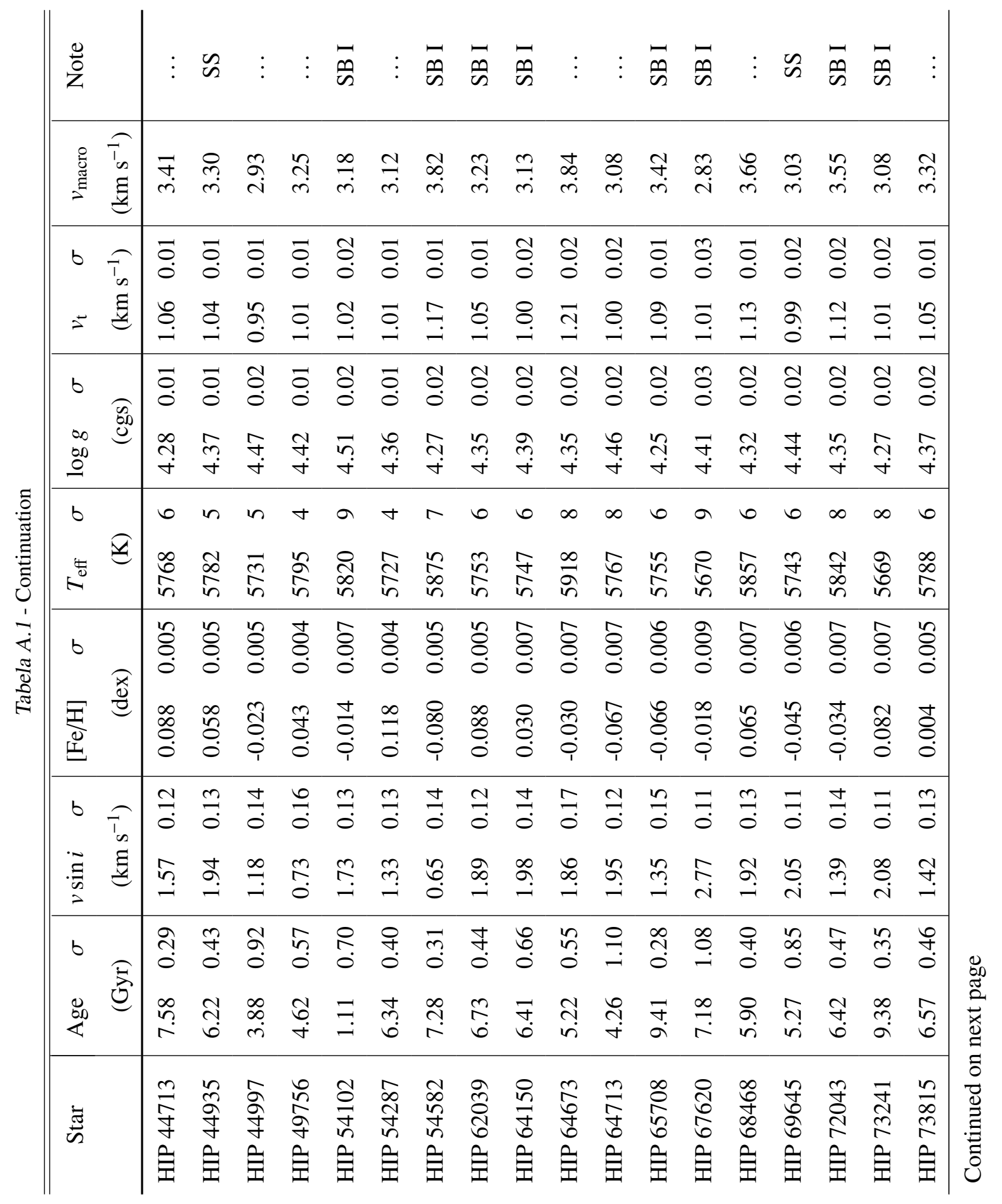




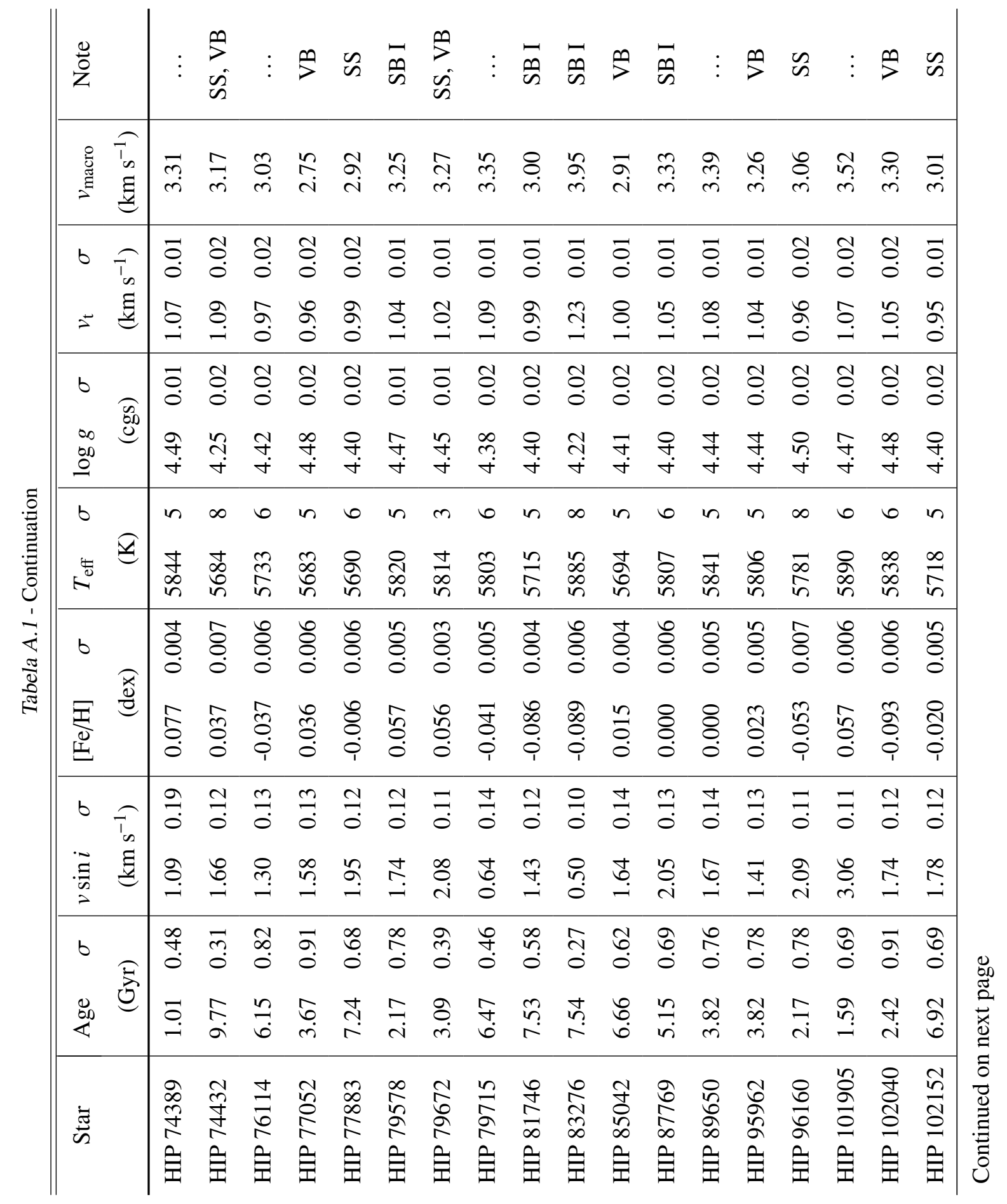




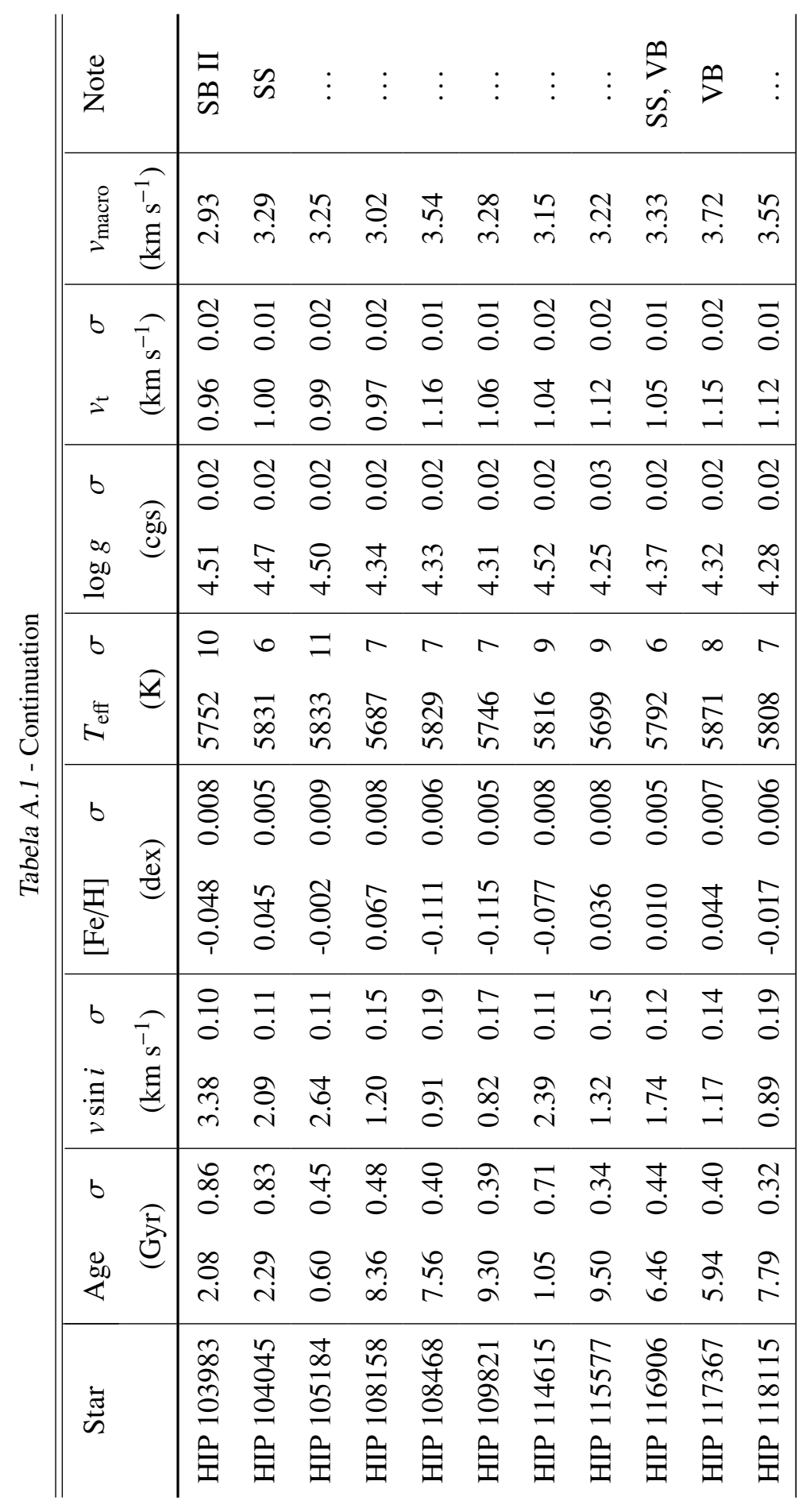




\section{Appendix B}

\section{First- and second-author articles}

Paper IV (dos Santos et al., 2016) of the Solar Twin Planet Search series of papers was originally published in Astronomy \& Astrophysics on 24 August 2016. Paper VII (dos Santos et al., 2017) of the series was accepted for publication in the Monthly Notices of the Royal Astronomical Society on August 24th 2017. The reproduction below is under permission from Astronomy \& Astrophysics, (c)ESO. 


\title{
The Solar Twin Planet Search
}

\section{The Sun as a typical rotator and evidence for a new rotational braking law for Sun-like stars $\star, \star \star$}

\author{
Leonardo A. dos Santos ${ }^{1,2}$, Jorge Meléndez ${ }^{1}$, José-Dias do Nascimento Jr. ${ }^{3,4}$, Megan Bedell², Iván Ramírez ${ }^{5}$, \\ Jacob L. Bean ${ }^{2}$, Martin Asplund ${ }^{6}$, Lorenzo Spina ${ }^{1}$, Stefan Dreizler ${ }^{7}$, Alan Alves-Brito ${ }^{8}$, and Luca Casagrande ${ }^{6}$ \\ 1 Universidade de São Paulo, Departamento de Astronomia do IAG/USP, Rua do Matão 1226, Cidade Universitária, \\ 05508-900 São Paulo, SP, Brazil \\ e-mail: leonardoags@usp.br \\ 2 University of Chicago, Department of Astronomy and Astrophysics, IL 60637, USA \\ 3 Universidade Federal do Rio Grande do Norte, 59072-970 Natal, RN, Brazil \\ 4 Harvard-Smithsonian Center for Astrophysics, Cambridge, MA 02138, USA \\ 5 University of Texas, McDonald Observatory and Department of Astronomy at Austin, USA \\ 6 The Australian National University, Research School of Astronomy and Astrophysics, Cotter Road, Weston, ACT 2611, Australia \\ 7 University of Göttingen, Institut für Astrophysik, Germany \\ 8 Universidade Federal do Rio Grande do Sul, Instituto de Física, Av. Bento Gonçalves 9500, 90650-002 Porto Alegre, RS, Brazil
}

Received 19 March 2016 / Accepted 20 June 2016

\begin{abstract}
Context. It is still unclear how common the Sun is when compared to other similar stars in regards to some of its physical properties, such as rotation. Considering that gyrochronology relations are widely used today to estimate ages of stars in the main sequence, and that the Sun is used to calibrate it, it is crucial to assess whether these procedures are acceptable.

Aims. We analyze the rotational velocities, limited by the unknown rotation axis inclination angle, of an unprecedented large sample of solar twins to study the rotational evolution of Sun-like stars, and assess whether the Sun is a typical rotator.

Methods. We used high-resolution $(R=115000)$ spectra obtained with the HARPS spectrograph and the $3.6 \mathrm{~m}$ telescope at La Silla Observatory. The projected rotational velocities for 81 solar twins were estimated by line profile fitting with synthetic spectra. Macroturbulence velocities were inferred from a prescription that accurately reflects their dependence with effective temperature and luminosity of the stars.

Results. Our sample of solar twins include some spectroscopic binaries with enhanced rotational velocities, and we do not find any nonspectroscopic binaries with unusually high rotation velocities. We verified that the Sun does not have a peculiar rotation, but the solar twins exhibit rotational velocities that depart from the Skumanich relation.

Conclusions. The Sun is a regular rotator when compared to solar twins with a similar age. Additionally, we obtain a rotational braking law that better describes the stars in our sample $\left(v \propto t^{-0.6}\right)$ in contrast to previous, often-used scalings.
\end{abstract}

Key words. Sun: rotation - stars: solar-type - stars: rotation - stars: fundamental parameters

\section{Introduction}

The Sun is the best-known star to astronomers and is commonly used as a template in the study of other similar objects. Yet, there are still some of its aspects that are not well understood and that are crucial for a better understanding of how stars and, consequently, how planetary systems and life evolve: how do the more complex physical parameters of a Sun-like star, such as rotation and magnetic activity, change with time? Is the Sun unique or

\footnotetext{
* Based on observations collected at the European Organisation for Astronomical Research in the Southern Hemisphere under ESO programs 188.C-0265, 183.D-0729, 292.C-5004, 077.C-0364, 072.C0488, 092.C-0721, 093.C-0409, 183.C-0972, 192.C-0852, 091.C0936, 089.C-0732, 091.C-0034, 076.C-0155, 185.D-0056, 074.C-0364, 075.C-0332, 089.C-0415, 60.A-9036, 075.C-0202, 192.C-0224, 090.C0421 and 088.C-0323.

$\star \star$ Full Table 3 is only available at the CDS

via anonymous ftp to cdsarc.u-strasbg. fr (130.79.128.5) or via http://cdsarc.u-strasbg.fr/viz-bin/qcat?J/A+A/592/A156
}

typical (i.e., an average Sun-like star)? If the Sun is common, it would mean that life does not require a special star for it to flourish, eliminating the need to evoke an anthropic reasoning to explain it.

In an effort to assess how typical the Sun is, Robles et al. (2008) compared 11 of its physical parameters with nearby stars, and concluded that the Sun is, in general, typical. Although they found it to be a slow-rotator against 276 F8 - K2 (within $\pm 0.1 M_{\odot}$ ) nearby stars, this result may be rendered inconclusive owing to unnacounted for noise that is caused by different masses and ages in their sample. Other studies have suggested that the Sun rotates either unusually slowly (Smith 1979; Leão et al. 2015) or regularly for its age (Soderblom 1983, 1985; Gray 1984; Gustafsson 1998; Barnes 2003), but none of these investigations comprised stars that are very similar to the Sun, therefore preventing a reliable comparison. In fact, with Kepler and CoRoT, it is now possible to obtain precise measurements of rotation periods, masses and ages of 
stars in a very homogeneous way (e.g., Ceillier et al. 2015; do Nascimento et al. 2012; Chaplin et al. 2014), but they generally lack high-precision stellar parameters, which are accessible through spectroscopy. The challenging nature of these observations limited ground-based efforts to smaller, but key stellar samples (e.g., Pizzolato et al. 2003; Strassmeier et al. 2012).

The rotational evolution of a star plays a crucial role in stellar interior physics and habitability. Previous studies proposed that rotation can produce extra mixing that is responsible for depleting the light elements $\mathrm{Li}$ and $\mathrm{Be}$ in their atmospheres (Pinsonneault et al. 1989; Charbonnel et al. 1994; Tucci Maia et al. 2015), which could explain the disconnection between meteoritic and solar abundances of Li (Baumann et al. 2010). Moreover, rotation is highly correlated with magnetic activity (e.g., Noyes et al. 1984; Soderblom et al. 1993; Baliunas et al. 1995; Mamajek \& Hillenbrand 2008), and this trend is key to understanding how planetary systems and life evolve in face of varying magnetic activity and energy outputs by solar-like stars during the main sequence (Guinan \& Engle 2009; Ribas et al. 2005; do Nascimento et al. 2016).

A theoretical treatment of rotational evolution from first principles is missing, so we often rely on empirical studies to make inferences about it. One of the pioneer efforts in this endeavor produced the well-known Skumanich relation $v \propto$ $t^{-1 / 2}$, where $v$ is the rotational velocity and $t$ is the stellar age (Skumanich 1972), which describes the rotational evolution of solar-type stars in the main sequence, and can be derived from the loss of angular momentum due to magnetized stellar winds (e.g., Kawaler 1988; Charbonneau 1992; Barnes 2003; Gallet \& Bouvier 2013). This relation sparked the development of gyrochronology, which consists in estimating stellar ages based on their rotation, and it was shown to provide a stellar clock as good as chromospheric ages (Barnes 2007). In Skumanich-like relations, however, the Sun generally falls on the curve (or plane, if we consider dependence on mass) defined by the rotational braking law by design. Thus it is of utmost importance to assess how common the Sun is to correctly calibrate it.

Subsequent studies have proposed modifications to this paradigm of rotation and chromospheric activity evolution (e.g., Soderblom et al. 1991; Pace \& Pasquini 2004), exploring rotational braking laws of the form $v \propto t^{-b}$. The formalism by Kawaler (1988) shows that this index $b$ can be related to the geometry of the stellar magnetic field, and that the Skumanich index $(b=1 / 2)$ corresponds to a geometry that is slightly more complex than a simple radial field. It also dictates the dependence of the angular momentum on the rotation rate and, in practice, it determines how early the effects of braking are felt by a model. Such prescriptions for rotational evolution have a general agreement for young ages up to the solar age (see Sood et al. 2016; Amard et al. 2016, and references therein), but the evolution for older ages still poses an open question. In particular, van Saders et al. (2016) suggested that stars undergo a weakened magnetic braking after they reach a critical value of the Rossby number, thus explaining the stagnation trend observed on the rotational periods of older Kepler stars.

In order to assess how typical the Sun is in its rotation, our study aims to verify whether the Sun follows the rotational evolution of stars that are very similar to it, which is an objective that is achieved by precisely measuring their rotational velocities and ages. We take advantage of an unprecedented large sample of solar twins (Ramírez et al. 2014) using high signal to noise $(S / N>500)$ and high-resolution $\left(R>10^{5}\right)$ spectra, which provides us with precise stellar parameters and is essential for the analysis that we perform (see Fig. 1 for an illustration of the subtle effects of rotation in stellar spectra of Sun-like stars).

\section{Working sample}

Our sample consists of bright solar twins in the Southern Hemisphere, which were mostly observed in our HARPS Large Program (ID: 188.C-0265) at the European Southern Observatory (ESO) that aimed to search for planetary systems around stars very similar to the Sun (Ramírez et al. 2014; Bedell et al. 2015; Tucci Maia et al. 2016, Papers I, II and III, respectively, of the series The Solar Twin Planet Search). These stars are loosely defined as those that have $T_{\text {eff }}, \log g$ and $[\mathrm{Fe} / \mathrm{H}]$ inside the intervals $\pm 100 \mathrm{~K}, \pm 0.1$ [cgs] and $0.1 \mathrm{dex}$, respectively, around the solar values. It has been shown that these limits guarantee $\sim 0.01$ dex precision in the relative abundances derived using standard model atmosphere methods and that the systematic uncertainties of that analysis are negligible within those ranges (Bedell et al. 2014; Biazzo et al. 2015; Saffe et al. 2015; Yana Galarza et al. 2016). In total, we obtained high-precision spectra for 73 stars and used data from 9 more targets observed in other programs; all of these overlapped the sample of 88 stars from Paper I. We used the spectrum of the Sun (reflected light from the Vesta asteroid) from the ESO program 088.C-0323, which was obtained with the same instrument and configuration as the solar twins.

The ages of the solar twin sample span between 0-10 Gyr and are presented in Table 3. They were obtained by Tucci Maia et al. (2016) using Yonsei-Yale isochrones (Yi et al. 2001) and probability distribution functions as described in Ramírez et al. (2013, 2014). Uncertainties are assumed to be symmetric. These ages are in excellent agreement with those obtained in Paper I, with a mean difference of $-0.1 \pm 0.2 \mathrm{Gyr}$ (see footnote 5 in Paper III). We adopted $4.56 \mathrm{Gyr}$ for the solar age (Bahcall et al. 1995). The other stellar parameters $\left(T_{\text {eff }}\right.$, $\log g,[\mathrm{Fe} / \mathrm{H}]$ and microturbulence velocities $v_{\mathrm{t}}$ ) were obtained by Ramírez et al. (2014). The stellar parameters of HIP 68468 and HIP 108158 were updated by Tucci Maia et al. (2016).

Our targets were observed at the HARPS spectrograph (Mayor et al. 2003), which is fed by ESO's $3.6 \mathrm{~m}$ telescope at La Silla Observatory. When available publicly, we also included all observations from other programs in our analysis in order to increase the signal-to-noise ratio $(\mathrm{S} / \mathrm{N})$ of our spectra. However, we did not use observations for 18 Sco (HIP 79672) from May 2009, owing to their instrumental artifacts, and we did not include observations post-HARPS upgrade (June 2015) when combining the spectra - they had a different shape in the red side, and since there were few observations, we chose not to use them to eliminate eventual problems with combination and normalization. Our initial plan was to use the observations from the MIKE spectrograph, as described by the Paper I. However, we decided to use the HARPS spectra due to its higher spectral resolving power.

The wavelength coverage for the observations ranged from 3780 to $6910 \AA$, with a spectral resolving power of $R=\lambda / \Delta \lambda=$ 115000 . Data reduction was performed automatically with the HARPS Data Reduction Software (DRS). Each spectrum was divided into two halves, corresponding to the mosaic of two detectors (one optimized for blue and other for red wavelengths). In this study we only worked with the red part (from 5330 to $6910 \AA$ ) because of its higher $\mathrm{S} / \mathrm{N}$ and the presence of cleaner lines. The correction for radial velocities was performed with 


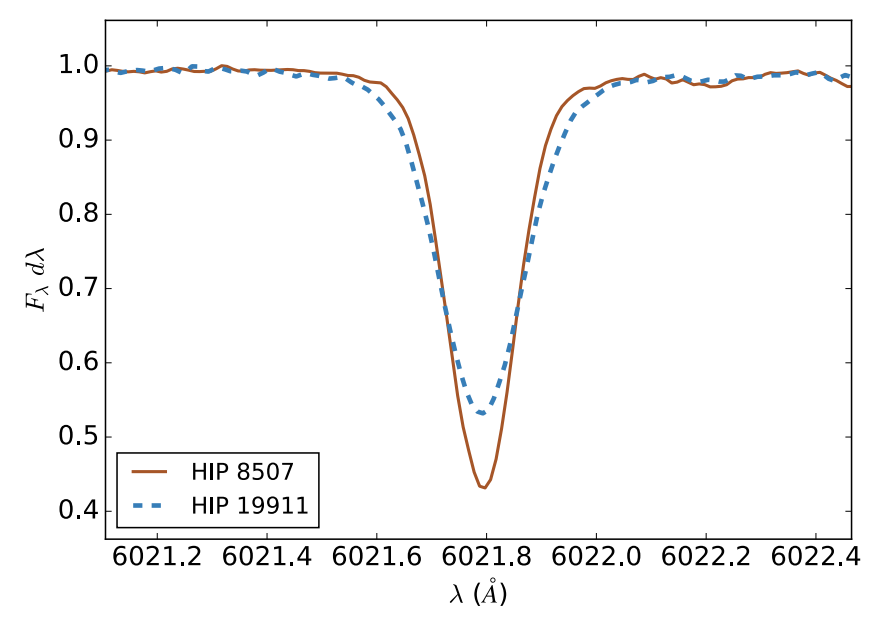

Fig. 1. Comparison of the spectral line broadening between two solar twins with different projected rotational velocities. The wider line corresponds to HIP 19911, with $v \sin i \approx 4.1 \mathrm{~km} \mathrm{~s}^{-1}$, and the narrower line comes from HIP 8507, with $v \sin i \approx 0.8 \mathrm{~km} \mathrm{~s}^{-1}$.

Table 1. Line list used in the projected stellar rotation measurements.

\begin{tabular}{cccccc}
\hline \hline $\begin{array}{c}\text { Wavelength } \\
(\AA)\end{array}$ & $Z$ & $\begin{array}{c}\text { Exc. pot. } \\
(\mathrm{eV})\end{array}$ & $\log (g f)$ & $\begin{array}{c}v_{\text {macro }}^{\odot} \\
\left(\mathrm{km} \mathrm{s}^{-1}\right)\end{array}$ & $\begin{array}{c}E W^{\circ} \\
(\AA)\end{array}$ \\
\hline 6027.050 & 26 & 4.076 & -1.09 & 3.0 & 0.064 \\
6151.618 & 26 & 2.176 & -3.30 & 3.2 & 0.051 \\
6165.360 & 26 & 4.143 & -1.46 & 3.1 & 0.045 \\
6705.102 & 26 & 4.607 & -0.98 & 3.6 & 0.047 \\
6767.772 & 28 & 1.826 & -2.17 & 2.9 & 0.079 \\
\hline
\end{tabular}

Notes. EW are the equivalent widths and $v_{\text {macro }}$ are the macroturbulence velocities measured as in Sect. 3.1.

the task dopcor from IRAF ${ }^{1}$, using the values obtained from the cross-correlation function $(\mathrm{CCF})$ of the pipeline. The different observations were combined with IRAF's scombine. The resulting average (of the sample) signal to noise ratio was 500 around $6070 \AA$. The red regions of the spectra were normalized with $\sim 30$ th order polynomial fits to the upper envelopes of the entire red range, using the task continuum on IRAF. We made sure that the continuum of the stars were consistent with the Sun's. Additionally, we verified that errors in the continuum determination introduce uncertainties in $v \sin i$ lower than $0.1 \mathrm{~km} \mathrm{~s}^{-1}$.

\section{Methods}

We analyzed five spectral lines, four due to Fe I and one to Ni I (see Table 1; equivalent widths were measured using the task splot in IRAF), which were selected for having low levels of contamination by blending lines. The rotational velocity of a star can be measured by estimating the spectral line broadening that is due to rotation. The rotation axes of the stars are randomly oriented, thus the spectroscopic measurements of rotational velocity are a function of the inclination angle $(v \sin i)$.

We estimate $v \sin i$ for our sample of solar twins using the 2014 version of MOOG Synth (Sneden 1973), adopting stellar

1 IRAF is distributed by the National Optical Astronomy Observatories, which are operated by the Association of Universities for Research in Astronomy, Inc., under cooperative agreement with the National Science Foundation.

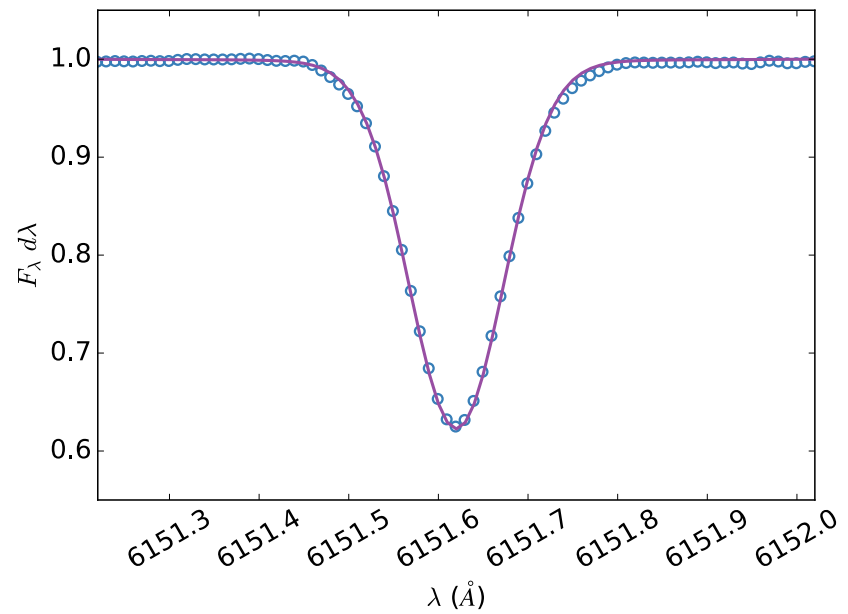

Fig. 2. Example of line profile fitting for the Fe I feature at $6151.62 \AA$ in the spectrum of the Sun. The continuous curve is the synthetic spectrum, and the open circles are the observed data.

atmosphere models by Castelli \& Kurucz (2004), with interpolations between models performed automatically by the Python package qoyllur-quipu ${ }^{2}$ (see Ramírez et al. 2014). The instrumental broadening is taken into account by the spectral synthesis. We used the stellar parameters from Tucci Maia et al. (2016) and microturbulence velocities from Ramírez et al. (2014). Macroturbulence velocities ( $v_{\text {macro }}$ ) were calculated by scaling the solar values, line by line (see Sect. 3.1). An estimation of the rotational velocities was performed with our own algorithm ${ }^{3}$ that makes automatic measurements for all spectral lines for each star. We applied fine-tuning corrections by eye for the nonsatisfactory automatic line profile fittings, and quote $v \sin i$ as the mean of the values measured for the five lines. See Sects. 3.1 and 3.2 for a detailed description on rotational velocities estimation and their uncertainties. Figure 2 shows an example of spectral line fitting for one feature in the Sun.

\subsection{Macroturbulence velocities}

We tested the possibility of measuring $v_{\text {macro }}$ (radial-tangential profile) simultaneously with $v \sin i$, but even when using the extremely high-resolution spectra of HARPS, it is difficult to disentangle these two spectral line broadening processes; this is probably because of the low values of these velocities. Macroturbulence has a stronger effect on the wings of the spectral lines, but our selection of clean lines still has some contamination that requires this high-precision work to be carried out by eye. Some stars show more contamination than others, complicating the disentaglement. Fortunately, the variation of macroturbulence with effective temperature and luminosity is smooth (Gray 2005), so that precise values of $v_{\text {macro }}$ could be obtained by a calibration. Thus we adopted a relation that fixes macroturbulence velocities to measure $v \sin i$ with high precision using an automatic code, which provides the additional benefits of reproducibility and lower subjectivity.

The macroturbulence velocity is known to vary for different spectral lines (Gray 2005), so for our high-precision analysis we do not adopt a single value for each star. Instead, we measure the $v_{\text {macro }}$ for the Sun in each of the spectral lines from Table 1

$\begin{array}{ll}2 & \text { Available at https://github.com/astroChasqui/q2 } \\ 3 & \text { Available at https://github.com/RogueAstro/PoWeRS }\end{array}$ 
and use these values to scale the $v_{\text {macro }}$ for all stars in our sample using the following equation ${ }^{4}$ :

$$
\begin{aligned}
v_{\text {macro }, \lambda}^{*}= & v_{\text {macro }, \lambda}^{\odot}-0.00707 T_{\text {eff }}+9.2422 \times 10^{-7} T_{\text {eff }}^{2} \\
& +10.0+k_{1}(\log g-4.44)+k_{2} \\
\equiv & f\left(T_{\text {eff }}\right)+k_{1}(\log g-4.44)+k_{2}
\end{aligned}
$$

where $v_{\text {macro }, \lambda}^{\odot}$ is the macroturbulence velocity of the Sun for a given spectral line, $T_{\text {eff }}$ and $\log g$ are the effective temperature and gravity of a given star, respectively, $k_{1}$ is a proportionality factor for $\log g$ and $k_{2}$ is a small correction constant.

This formula is partly based on the relation derived by Meléndez et al. (2012; Eq. (E.1) in their paper) from the trend of macroturbulence with effective temperature in solar-type stars described by Gray (2005). The $\log g$-dependent term (a proxy for luminosity) comes from the empirical relation derived by Doyle et al. (2014; Eq. (8) in their paper), and is based on spectroscopic measurements of $v_{\text {macro }}$ of Kepler stars, which were disentangled from $v \sin i$ using asteroseismic estimates of the projected rotational velocities. Doyle et al. obtained a value for the proportionality factor $k_{1}$ of -2.0 . Their uncertainties on $v_{\text {macro }}$, however, were on the order of $1.0 \mathrm{~km} \mathrm{~s}^{-1}$. Thus, we decided to derive our own values of $k_{1}$ and $k_{2}$ by simultaneously measuring $v_{\text {macro }}$ and $v \sin i$ of a subsample of solar twins.

This subsample was chosen to contain only single stars or visual binaries mostly in the extremes of $\log g$ (4.25-4.52) in our entire sample. We assume these values have a linear relationship with $v_{\text {macro }}$ inside this short interval of $\log g$. We used as a first guess the values of $v \sin i$ and $v_{\text {macro }}$ from a previous, cruder estimation we made, and performed line profile fits by eye using MOOG Synth. The velocities in Table 2 are the median of the values measured for each line and their standard error. These $v \sin i$ are not consistently measured in the same way as the final results. The rotational velocity broadening was calculated by our own code (see Sect. 3.2 for details). By performing a linear fit in the $v_{\text {macro }}-f\left(T_{\text {eff }}\right)$ versus $\log g-4.44$ relation ( $f$ comprises all the $T_{\text {eff }}$-dependent terms, the macroturbulence velocity of the Sun and the known constant on Eq. (1)), we obtain that $k_{1}=-1.81 \pm 0.26$ and $k_{2}=-0.05 \pm 0.03$ (see Fig. 3). For the stars farthest from the Sun in $\log g$ from our sample, these values of $k_{1}$ and $k_{2}$ would amount to differences of up to $\pm 0.4 \mathrm{~km} \mathrm{~s}^{-1}$ in their macroturbulence velocities, therefore it is essential to consider the luminosity effect on $v_{\text {macro }}$ for accurate $v \sin i$ determinations.

To obtain the macroturbulence velocities for the Sun to use in Eq. (1), we forced the rotational velocity of the Sun to $1.9 \mathrm{~km} \mathrm{~s}^{-1}$ (Howard \& Harvey 1970), and then estimated values of $v_{\text {macro, } \lambda}^{\odot}$ by fitting each line profile using MOOG Synth, and the results are shown in Table 1 . We estimated the error in determining $v_{\text {macro }, \lambda}^{\odot}$ to be $\pm 0.1 \mathrm{~km} \mathrm{~s}^{-1}$. Since Eq. (1) is an additive scaling, the error for $v_{\text {macro }}$ of all stars is the same as in the Sun. The uncertainties in stellar parameters have contributions that are negligible compared to the ones introduced by the error in $v_{\text {macro }}$.

\subsection{Rotational velocities}

Our code takes as input the list of stars and their parameters (effective temperature, surface gravity, metallicity and microturbulence velocities obtained on Paper I), their spectra and the spectral line list in MOOG-readable format. For each line in a given

\footnotetext{
4 In the future, it should be possible to calibrate macroturbulence velocities using 3D hydrodynamical stellar atmosphere models (e.g., Magic et al. 2013) by using predicted 3D line profiles (without rotational broadening) as observations and determine which value of $v_{\text {macro }}$ is needed to reproduce them with $1 \mathrm{D}$ model atmospheres.
}

Table 2. Simultaneous measurements of rotational and macroturbulence velocities of stars in the extremes of $\log g$ from our sample of solar twins.

\begin{tabular}{lcccc}
\hline \hline Star & $\begin{array}{c}v \sin i \\
\left(\mathrm{~km} \mathrm{~s}^{-1}\right)\end{array}$ & $\begin{array}{c}v_{\text {macro }} \\
\left(\mathrm{km} \mathrm{s}^{-1}\right)\end{array}$ & $T_{\text {eff }}$ & $\log g$ \\
\hline HIP 115577 & $0.95 \pm 0.05$ & $3.35 \pm 0.09$ & 5699 & 4.25 \\
HIP 65708 & $1.20 \pm 0.09$ & $3.55 \pm 0.08$ & 5755 & 4.25 \\
HIP 74432 & $1.40 \pm 0.03$ & $3.35 \pm 0.08$ & 5684 & 4.25 \\
HIP 118115 & $1.40 \pm 0.10$ & $3.43 \pm 0.09$ & 5808 & 4.28 \\
HIP 68468 & $1.75 \pm 0.07$ & $3.70 \pm 0.08$ & 5857 & 4.32 \\
HIP 41317 & $1.55 \pm 0.03$ & $3.10 \pm 0.06$ & 5700 & 4.38 \\
Sun & $1.75 \pm 0.07$ & $3.30 \pm 0.06$ & 5777 & 4.44 \\
HIP 105184 & $2.50 \pm 0.03$ & $3.21 \pm 0.08$ & 5833 & 4.50 \\
HIP 10175 & $1.55 \pm 0.06$ & $3.05 \pm 0.08$ & 5738 & 4.51 \\
HIP 114615 & $2.20 \pm 0.03$ & $3.25 \pm 0.08$ & 5816 & 4.52 \\
HIP 3203 & $3.90 \pm 0.03$ & $3.40 \pm 0.10$ & 5850 & 4.52 \\
\hline
\end{tabular}

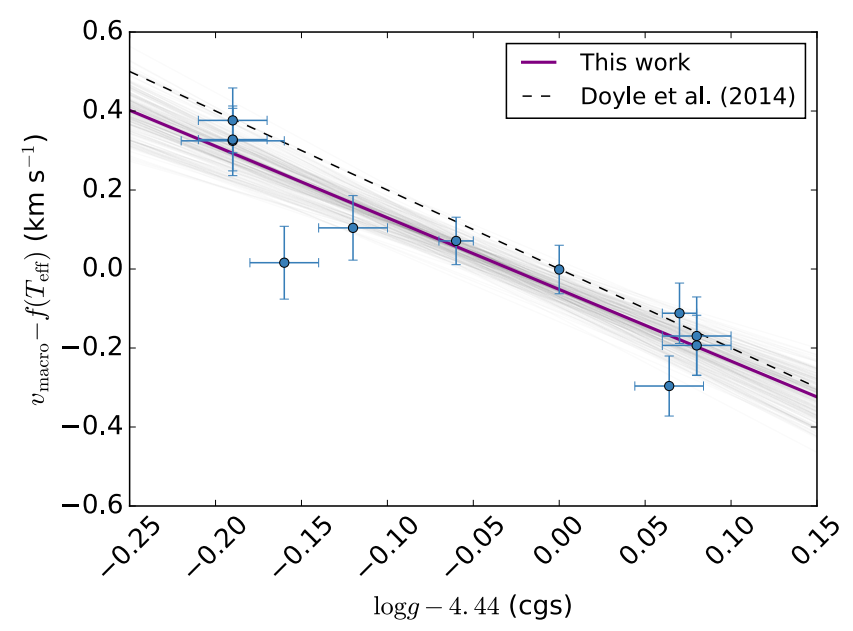

Fig. 3. Linear relation between $v_{\text {macro }}$ and $\log g$ (a proxy for luminosity) for the stars on Table 2. See the definition of $f\left(T_{\text {eff }}\right)$ in Sect. 3.1. The orange continuous line represents our determination of a proportionality coefficient of -1.81 and a vertical shift of $-0.05 \mathrm{~km} \mathrm{~s}^{-1}$. The black dashed line is the coefficient found by Doyle et al. (2014). The light gray region is a composition of 200 curves with parameters drawn from a multivariate Gaussian distribution. The Sun is located at the origin.

star, the code automatically corrects the spectral line shift and the continuum. The first is performed by fitting a second-order polynomial to the kernel of a line and estimating the distance of the observed line center from the laboratory value. Usually, the spectral line shift corrections were on the order of $10^{-2} \AA$, corresponding to $0.5 \mathrm{~km} \mathrm{~s}^{-1}$ in the wavelength range we worked on. This is a reasonable shift that likely arises from a combination of granulation and gravitational redshift effects, which are of similar magnitude. The continuum correction for each line is defined as the value of a multiplicative factor that sets the highest flux inside a radius of $2.5 \AA$ around the line center to 1.0. The multiplicative factor usually has a value inside the range $1.000 \pm 0.002$.

The code starts with a range of $v \sin i$ and abundances and optimizes these two parameters through a series of iterations that measure the least-squares difference between the observed line and the synthetic line (generated with MOOG synth). Convergence is achieved when the difference between the best and previous solutions, for both $v \sin i$ and abundance, is less than $1 \%$. 
L. A. dos Santos et al.: The Solar Twin Planet Search. IV.

Table 3. Ages, the measured $v \sin i$, and stellar parameters of the solar twins and the Sun.

\begin{tabular}{|c|c|c|c|c|c|c|c|c|c|c|c|c|c|c|}
\hline Star & $\begin{array}{l}\text { Age } \\
(C\end{array}$ & $\left.{ }^{\sigma}\right)^{\sigma}$ & $\begin{array}{r}v \sin i \\
\quad(\mathrm{kn}\end{array}$ & & $\begin{array}{r}{[\mathrm{Fe} / \mathrm{H}]} \\
(\mathrm{d}\end{array}$ & $\sigma$ & $\begin{array}{l}T_{\text {eff }} \\
\quad(\mathrm{K}\end{array}$ & $\sigma$ & $\begin{array}{r}\log g \\
\text { (c }\end{array}$ & & $\begin{array}{l}v_{\mathrm{t}} \\
(\mathrm{km}\end{array}$ & $\begin{array}{r}\sigma \\
-1\end{array}$ & $\begin{array}{c}v_{\text {macro }} \\
\left(\mathrm{km} \mathrm{s}^{-1}\right)\end{array}$ & Note \\
\hline Sun & 4.56 & & 2.04 & 0.12 & 0.000 & $\ldots$ & 5777 & & 4.44 & & 1.00 & & 3.20 & SS \\
\hline HIP 1954 & 4.87 & 0.97 & 1.79 & 0.13 & -0.068 & 0.006 & 5717 & 5 & 4.46 & 0.02 & 0.96 & 0.02 & 2.90 & \\
\hline HIP 3203 & 0.99 & 0.66 & 3.82 & 0.11 & -0.087 & 0.008 & 5850 & 10 & 4.52 & 0.02 & 1.16 & 0.02 & 3.27 & SS \\
\hline HIP 4909 & 1.23 & 0.77 & 4.01 & 0.11 & +0.028 & 0.008 & 5854 & 10 & 4.50 & 0.02 & 1.12 & 0.02 & 3.33 & SS \\
\hline HIP 5301 & 6.49 & 0.67 & 2.00 & 0.12 & -0.064 & 0.004 & 5728 & 5 & 4.42 & 0.02 & 0.97 & 0.01 & 3.01 & SS \\
\hline HIP 6407 & 1.49 & 0.66 & 2.30 & 0.13 & -0.068 & 0.007 & 5764 & 8 & 4.52 & 0.01 & 0.97 & 0.02 & 2.96 & SB I \\
\hline HIP 7585 & 3.29 & 0.51 & 1.90 & 0.15 & +0.095 & 0.005 & 5831 & 5 & 4.43 & 0.01 & 1.02 & 0.01 & 3.37 & $\ldots$ \\
\hline HIP 8507 & 3.63 & 0.94 & 0.77 & 0.15 & -0.096 & 0.006 & 5725 & 6 & 4.49 & 0.02 & 0.99 & 0.02 & 2.88 & $\ldots$ \\
\hline HIP 9349 & 1.43 & 0.76 & 2.25 & 0.11 & +0.009 & 0.007 & 5810 & 8 & 4.50 & 0.02 & 1.07 & 0.02 & 3.16 & $\ldots$ \\
\hline HIP 10175 & 1.82 & 0.65 & 1.83 & 0.11 & -0.007 & 0.005 & 5738 & 7 & 4.51 & 0.01 & 0.96 & 0.01 & 2.89 & $\ldots$ \\
\hline HIP 10303 & 5.48 & 0.56 & 0.77 & 0.16 & +0.106 & 0.004 & 5725 & 4 & 4.40 & 0.01 & 0.98 & 0.01 & 3.04 & $\ldots$ \\
\hline
\end{tabular}

Notes. SS are stars belonging to the selected sample; SB I are single-lined spectroscopic binaries. This table is available in its entirety in machinereadable format at the CDS. A portion is shown here for guidance regarding its form and content.

Additionally, the code also forces at least ten iterations to avoid falling into local minima.

One of the main limitations of MOOG Synth for our analysis is that it has a "quantized" behavior for $v \sin i$ : the changes in the synthetic spectra occur most strongly in steps of $0.5 \mathrm{~km} \mathrm{~s}^{-1}$. This behavior is not observed in varying the macroturbulence velocities. Therefore, we had to incorporate a rotational broadening routine in our code that was separated from MOOG. We used the Eq. (18.14) from Gray (2005), in velocity space, to compute the rotational profile ${ }^{5}$

$G(v)=\frac{2(1-\epsilon)\left[1-\left(v / v_{\mathrm{L}}\right)^{2}\right]^{1 / 2}+\frac{1}{2} \pi \epsilon\left[1-\left(v / v_{\mathrm{L}}\right)^{2}\right]}{\pi v_{\mathrm{L}}(1-\epsilon / 3)}$,

where $v_{\mathrm{L}}$ is the projected rotational velocity and $\epsilon$ is the limb darkening coefficient (for which we adopt the value 0.6). The rotational profile $G(v)$ is then convolved with the MOOG synthetic profiles, which were generated with $v \sin i=0$.

The total uncertainties in rotational velocities are obtained from the quadratic sum of the standard error of the five measurements and an uncertainty of $0.1 \mathrm{~km} \mathrm{~s}^{-1}$ introduced by the error in macroturbulence velocities. Systematic errors in the calculation of $v_{\text {macro, } \lambda}$ for the stars do not significantly contribute to the $v \sin i$ uncertainties. The rotational velocities we measured and their uncertainties are reproduced in Table 3.

Some of the stars in the sample show very low rotational velocities, most probably owing to the effect of projection (see left panel of Fig. 5). The achieved precision is validated by comparison with the values of the full width at half maximum (FWHM) measured by the cross-correlation function (CCF) from the data reduction pipeline, with the effects of macroturbulence subtracted (see Fig. 4). The spectroscopic binary star HIP 103983 has an unusually high $v \sin i$ when compared to the CCF FWHM, and a verification of its spectral line profiles reveals the presence of distortions, which are the most probably caused by mismeasurement of rotational velocity (contamination of the combined spectrum by a companion; observations range from October 2011 to August 2012). We obtained a curve fit for the $v \sin i$ versus CFF FWHM $\left(\mathrm{km} \mathrm{s}^{-1}\right)$ using a similar relation as used by Melo et al. (2001),

\footnotetext{
5 This is the same recipe adopted by the radiative transfer code MOOG.
}

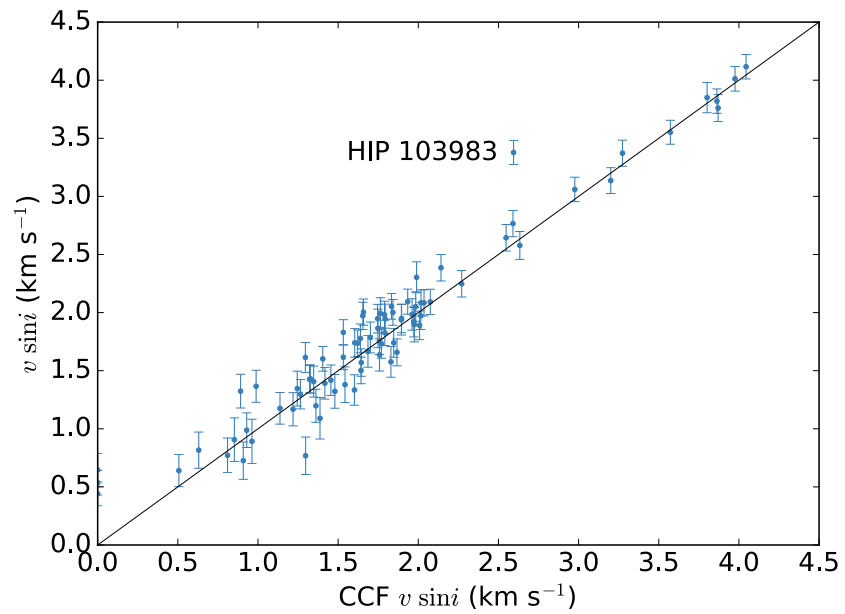

Fig. 4. Comparison between our estimated values of $v \sin i$ ( $y$-axis) and those inferred from the cross-correlation funcion FWHM ( $x$-axis). The spread around the $1: 1$ relation (black line) is $\sigma=0.20 \mathrm{~km} \mathrm{~s}^{-1}$.

Pace \& Pasquini (2004), Hekker \& Meléndez (2007), which resulted in the following calibration: $v \sin i=$ $\sqrt{(0.73 \pm 0.02)\left[F W H M^{2}-v_{\text {macro }}^{2}-(5.97 \pm 0.01)^{2}\right]} \mathrm{km} \mathrm{s}^{-1}$ (estimation performed with the MCMC code emcee ${ }^{6}$ Foreman-Mackey et al. 2013). The scatter between the measured $v \sin i$ and those estimated from CCF is $\sigma=0.20 \mathrm{~km} \mathrm{~s}^{-1}$ (excluding the outlier HIP 103983). The typical uncertainty in the rotational velocities we obtain with our method, that is, line profile fitting with extreme high-resolution spectra, is $0.12 \mathrm{~km} \mathrm{~s}^{-1}$, which implies that the average error of the CCF FWHM $v \sin i$ scaling is $0.16 \mathrm{~km} \mathrm{~s}^{-1}$. This average error could be significantly higher if the broadening by $v_{\text {macro }}$ is not accounted for.

\section{Binary stars}

We identified 16 spectroscopic binaries (SB) in our sample of 81 solar twins by analyzing their radial velocities; some of

\footnotetext{
6 Available at http://dan . iel. fm/emcee/current/
} 

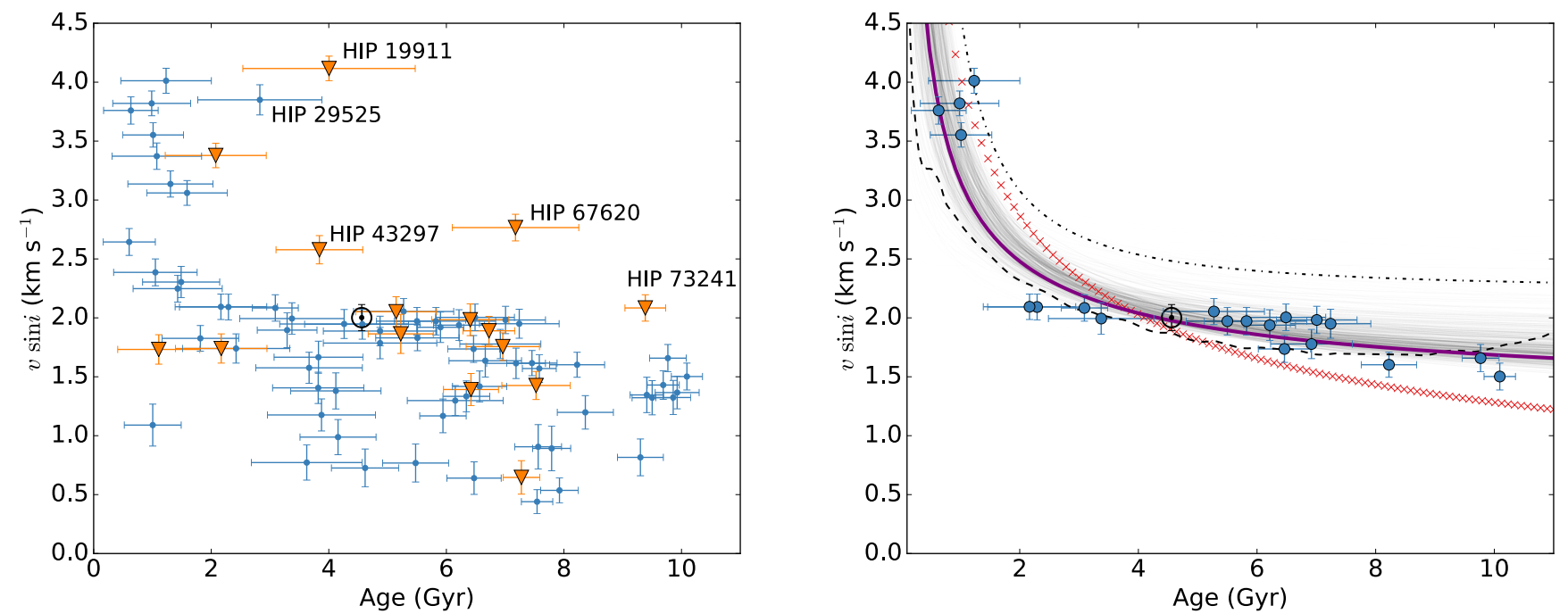

Fig. 5. Projected rotational velocity of solar twins as a function of their age. The Sun is represented by the symbol $\odot$. Left panel: all stars of our sample; the orange triangles are spectroscopic binaries, blue circles are the selected sample and the blue dots are the remaining nonspectroscopic binaries. Right panel: the rotational braking law; the purple continuous curve is our relation inferred from fitting the selected sample (blue circles) of solar twins with the form $v \sin i=v_{\mathrm{f}}+m t^{-b}$, where $t$ is the stellar age, and the fit parameters are $v_{\mathrm{f}}=1.224 \pm 0.447, m=1.932 \pm 0.431$, and $b=0.622 \pm 0.354$, with $v_{\mathrm{f}}$ and $b$ highly and positively correlated. The light gray region is composed of 300 curves that are created with parameters drawn from a multivariate Gaussian distribution defined by the mean values of the fit parameters and their covariance matrix. Skumanich's law $\left(\right.$ red $\times$ symbols, calibrated for $v_{\text {rot }}^{\odot}=1.9 \mathrm{~km} \mathrm{~s}^{-1}$ ) and the rotational braking curves proposed by do Nascimento et al. (2014, black dashed curve, smoothed) and Pace \& Pasquini (2004, black dot-dashed curve) are plotted for comparison.

these stars are reported as binaries by Tokovinin $(2014 \mathrm{a}, \mathrm{b})$, Mason et al. (2001), Baron et al. (2015). We did not find previous reports of multiplicity for the stars HIP 30037, HIP 62039 and HIP 64673 in the literature. Our analysis of variation in the HARPS radial velocities suggest that the first two are probable SBs, while the latter is a candidate. No binary shows a doublelined spectrum, but HIP 103983 has distortions that could be from contamination by a companion. The star HIP 64150 is a Sirius-like system with a directly observed white dwarf companion (Crepp et al. 2013; Matthews et al. 2014). The sample from Paper I contains another SB, HIP 109110, for which we could not reliably determine the $v \sin i$ because of strong contamination in the spectra, which is possibly caused by a relatively bright companion. Thus, we did not include this star in our sample.

Of these 16 spectroscopic binaries, at least four of them (HIP 19911, 43297, 67620 and 73241) show unusually high $v \sin i$ (see the left panel of Fig. 5). These stars also present other anormalities, such as their [Y/Mg] abundances (Tucci Maia et al. 2016) and magnetic activity (Ramírez et al. 2014; Freitas et al., in prep.). The solar twin blue straggler HIP 10725 (Schirbel et al. 2015), which is not included in our sample, also shows a high $v \sin i$ for its age. We find that five of the binaries have rotational velocities below the expected for Sun-like stars, but this is most likely an effect of projection of the rotational axes of the stars. For the remaining binaries, which follow the rotational braking law, it is again difficult to disentangle this behavior from the $\sin i$, and a statistical analysis is precluded by the low numbers involved. Tidal interactions between companions that could potentially enhance rotation depend on binary separation, which is unknown for most of these stars. They should be regular rotators, since they do not show anormalities in chromospheric activity (Freitas et al., in prep.) or [Y/Mg] abundances (Tucci Maia et al. 2016).

Based on the information that at least $25 \%$ of the spectroscopic binaries in our sample show higher rotational velocities than expected for single stars, we conclude that stellar multiplicity is an important enhancer of rotation in Sun-like stars. Blue stragglers are expected to have a strong enhancement on rotation owing to injection of angular momentum from the donor companion.

\section{The rotational braking law}

We removed from this analysis all the spectroscopic binaries in order to correctly constrain the rotational braking. The nonSB HIP 29525 displays a $v \sin i$ that is much higher than expected $\left(3.85 \pm 0.13 \mathrm{~km} \mathrm{~s}^{-1}\right)$, but it is likely that this is the result of an overestimated isochronal age $(2.83 \pm 1.06 \mathrm{Gyr})$. We decided to not include HIP 29525 in the rotational braking determination because it is a clear outlier in our results. Maldonado et al. (2010) found X-ray and chromospheric ages of 0.55 and 0.17 Gyr, respectively, for HIP 29525. We then divided the remaining 65 stars and the Sun in bins of 2 Gyr, and removed all the stars that were below the 70th percentile of $v \sin i$ in each bin from this sample. Such a procedure can be justified because we verified that $30 \%$ of the stars should have $\sin i$ above 0.9 by doing a simple simulation with angles $i$ drawn from a flat distribution between 0 and $\pi / 2$. This allowed us to select the stars that had the highest chance of having $\sin i$ above 0.9 . In total, 21 solar twins and the Sun compose what we hereafter reference as the selected sample. Albeit this subsample is smaller, it has the advantage of mostly removing uncertainties on the inclination angle of the stellar rotation axes 7 . We stress that the only reason we can select the most probable edge-on rotating stars $(i=\pi / 2)$ is because we have a large sample of solar twins in the first place.

We then proceeded to fit a general curve to the selected sample (see Fig. 5) using the method of orthogonal distance

7 This procedure can also allow for unusually fast-rotating stars (although rare) with $\sin i$ below 0.9 to leak into the subsample. 
regression (ODR, Boggs \& Rogers 1990), which takes the uncertainties on both $v \sin i$ and ages into account. This curve is a power law plus constant of the form $v=v_{\mathrm{f}}+m t^{-b}$ (the same chromospheric activity and $v \sin i$ vs. age relation used by Pace $\&$ Pasquini 2004; Guinan $\&$ Engle 2009), with $v$ (rotational velocity) and $v_{\mathrm{f}}$ (asymptotic velocity) in $\mathrm{km} \mathrm{s}^{-1}$ and $t$ (age) in Gyr.

We find that the best-fit parameters are $v_{\mathrm{f}}=1.224 \pm 0.447$, $m=1.932 \pm 0.431$, and $b=0.622 \pm 0.354$ (see right panel of Fig. 5). These large uncertainties are likely due to i) the strong correlation between $v_{\mathrm{f}}$ and $b$; and ii) the relatively limited number of data points between 1 and 4 Gyr, where the parameters are most effective in changing the values of $v$. This limitation is also present in past studies (e.g., van Saders et al. 2016; Barnes 2003; Pace \& Pasquini 2004; Mamajek \& Hillenbrand 2008; García et al. 2014; Amard et al. 2016). On the other hand, our sample is the largest comprising solar twins and, therefore, should produce more reliable results. With more data points, we could be able to use $1 \mathrm{Gyr}$ bins instead of $2 \mathrm{Gyr}$ in order to select the fastest rotating stars, which would result in a better subsample for constraining the rotational evolution for young stars.

The relation we obtain is in contrast with some previous studies on modeling the rotational braking (Barnes 2001, 2003; Lanzafame \& Spada 2015) which either found or assumed that the Skumanich law explains well the rotational braking of Sunlike stars. The conclusions by van Saders et al. (2016) limit the range of validation up to approximately the solar age (4 Gyr) for stars with solar mass. When we enforce the Skumanich powerlaw index $b=1 / 2$, we obtain a worse fit between the ages 2 and 4 Gyr and, not surprisingly, after the solar age as well.

Our data and the rotational braking law that results from them show that the Sun is a normal star regarding its rotational velocity when compared to solar twins. However, they do not agree with the regular Skumanich law (Barnes 2007, red $\times$ symbols in Fig. 5). We find a better agreement with the model proposed by do Nascimento et al. (2014, black dashed curve in Fig. 5), especially for stars older than 2 Gyr. This model is thoroughly described in Appendix A of do Nascimento et al. (2012). In summary, this model uses an updated treatment of the instabilities that are relevant to the transport of angular momentum, according to Zahn (1992) and Talon \& Zahn (1997), with an initial angular momentum for the Sun $J_{0}=1.63 \times 10^{50} \mathrm{~g} \mathrm{~cm}^{2} \mathrm{~s}^{-1}$. The rotational braking curve that corresponds to the model by do Nascimento et al. (2014) is computed using the output radii of the model, which vary from $\sim 1 R_{\odot}$ at the current solar age to $1.57 R_{\odot}$ at the age of $11 \mathrm{Gyr}$, and it changes significantly if we use a constant radius $R=1 R_{\odot}$. This results in a more Skumanich-like rotational braking.

Our result agrees with the chromospheric activity versus age behavior for solar twins obtained by Ramírez et al. (2014), in which a steep decay of the $R_{\mathrm{HK}}^{\prime}$ index during the first 4 Gyr was deduced (see Fig. 11 in their paper). The study by Pace \& Pasquini (2004) also suggests a steeper power-law index ( $b=1.47)$ than Skumanich's $\left(b_{\mathrm{S}}=1 / 2\right)$ in the rotational braking law derived from young open clusters, the Sun and M 67. As seen in Fig. 5, however, their relation significantly overestimates the rotational velocities of stars, especially for those older than 2 Gyr. This is most probably caused by other line broadening processes, mainly the macroturbulence, which were not considered in that study. As we saw in Sect. 3.1, those processes introduce important effects that are sometimes larger than the rotational broadening. Moreover, a CCF-only analysis tends to produce more spread in the $v \sin i$ than the more detailed analysis we used.
The rotational braking law we obtain produces a similar outcome to that achieved by van Saders et al. (2016) for stars older than the Sun, that is, a weaker rotational braking law after solar age than previously suggested. Our data also requires a different power-law index than the Skumanich index for stars younger than the Sun, accounting for an earlier decay of rotational velocities up to 2 Gyr.

The main-sequence spin-down model by Kawaler (1988) states that, for constant moment of inertia and radius during the main sequence, we would have

$v_{\mathrm{eq}} \propto t^{-3 /(4 a n)}$,

where $v_{\text {eq }}$ is the rotational velocity at the equator and $a$ and $n$ are parameters that measure the dependence on rotation rate and radius, respectively (see Eqs. (7), (8) and (12) in their paper). If we assume a dipole geometry for the stellar magnetic field $\left(B_{\mathrm{r}} \propto B_{0} r^{3}\right)$, then $n=3 / 7$. Furthermore, assuming that $a=1$, then Eq. (3) results in $v_{\text {eq }} \propto t^{-7 / 4}=t^{-1.75}$. The Skumanich law $\left(v_{\mathrm{eq}} \propto t^{-0.5}\right)$ is recovered for $n=3 / 2$, which is close to the case of a purely radial field $\left(n=2, v_{\mathrm{eq}} \propto t^{-0.38}\right)$. A more extensive exploration of the configuration and evolution of magnetic fields of solar twins is outside the scope of this paper, but our results suggest that the rotational rotational braking we observe on this sample of solar twins stems from a magnetic field with an intermediate geometry between dipole and purely radial.

\section{Conclusions}

We analyzed the rotational velocities of 81 bright solar twins in the Southern Hemisphere and the Sun using extremely highresolution spectra. Radial velocities revealed that our sample contained 16 spectroscopic binaries, 3 of which (HIP 30037 , 62039,64673 ) were not listed as such in the literature. At least 5 of these stars show an enhancement on their measured $v \sin i$, which is probably caused by interaction with their close-by companions. They also present other anomalies in chemical abundances and chromospheric activities. We did not clearly identify nonspectroscopic binary stars with unusually high rotational velocities for their age.

We selected a subsample of stars with higher chances of having their rotational axis inclination close to $\pi / 2$ (almost edge-on) in order to better constrain the rotational evolution of the solar twins. We opted to use carefully measured isochronal ages for these stars because it is the most reliable method available for this sample. We finally conclude that the Sun seems to be a common rotator, within our uncertainties, when compared to solar twins, therefore it can be used to calibrate stellar models.

Moreover, we have found that the Skumanich law does not describe well the rotation evolution for solar twins observed in our data, which is a discrepancy that is stronger after the solar age. Therefore, we propose a new rotational braking law that supports the weakened braking after the age of the Sun, and comes with a earlier decay in rotational velocities up to $2 \mathrm{Gyr}$ than the classical Skumanich's law. Interestingly, it also reveals an evolution that is more similar to the magnetic activity evolution observed in Sun-like stars, which sees a steep decay in the first $3 \mathrm{Gyr}$ and flattens near the solar age. Additionally, we suggest that more high-precision spectroscopic observations of solar twins younger and much older than the Sun could help us better constrain the rotational evolution of solar-like stars.

Acknowledgements. L.d.S. thanks CAPES and FAPESP, grants No. 2014/269081 and 2016/01684-9 for support. J.M. thanks FAPESP (2012/24392-2) for support. L.S. acknowledges support by FAPESP (2014/15706-9). We also would 
like to thank the anonymous referee for valuable comments that significantly improved this manuscript.

\section{References}

Amard, L., Palacios, A., Charbonnel, C., Gallet, F., \& Bouvier, J. 2016, A\&A, 587, A105

Bahcall, J. N., Pinsonneault, M. H., \& Wasserburg, G. J. 1995, Rev. Mod. Phys., 67, 781

Baliunas, S. L., Donahue, R. A., Soon, W. H., et al. 1995, ApJ, 438, 269

Barnes, S. A. 2001, ApJ, 561, 1095

Barnes, S. A. 2003, ApJ, 586, 464

Barnes, S. A. 2007, ApJ, 669, 1167

Baron, F., Lafrenière, D., Artigau, É., et al. 2015, ApJ, 802, 37

Baumann, P., Ramírez, I., Meléndez, J., Asplund, M., \& Lind, K. 2010, A\&A, 519, A87

Bedell, M., Meléndez, J., Bean, J. L., et al. 2014, ApJ, 795, 23

Bedell, M., Meléndez, J., Bean, J. L., et al. 2015, A\&A, 581, A34

Biazzo, K., Gratton, R., Desidera, S., et al. 2015, A\&A, 583, A135

Boggs, P. T., \& Rogers, J. E. 1990, Contemporary Mathematics, 112, 183

Castelli, F., \& Kurucz, R. L. 2004, ArXiv e-prints [arXiv: astro-ph/0405087]

Ceillier, T., García, R. A., Salabert, D., \& Mathur, S. 2015, in EPJ Web Conf., 101, 06016

Chaplin, W. J., Basu, S., Huber, D., et al. 2014, ApJS, 210, 1

Charbonneau, P. 1992, in Cool Stars, Stellar Systems, and the Sun, eds. M. S. Giampapa, \& J. A. Bookbinder, ASP Conf. Ser., 26, 416

Charbonnel, C., Vauclair, S., Maeder, A., Meynet, G., \& Schaller, G. 1994, A\&A, 283, 155

Crepp, J. R., Johnson, J. A., Howard, A. W., et al. 2013, ApJ, 774, 1

do Nascimento, J.-D., da Costa, J. S., \& Castro, M. 2012, A\&A, 548, L1

do Nascimento, Jr., J.-D., García, R. A., Mathur, S., et al. 2014, ApJ, 790, L23

do Nascimento, Jr., J.-D., Vidotto, A. A., Petit, P., et al. 2016, ApJ, 820, L15

Doyle, A. P., Davies, G. R., Smalley, B., Chaplin, W. J., \& Elsworth, Y. 2014 MNRAS, 444, 3592

Foreman-Mackey, D., Hogg, D. W., Lang, D., \& Goodman, J. 2013, PASP, 125, 306

Gallet, F., \& Bouvier, J. 2013, A\&A, 556, A36

García, R. A., Ceillier, T., Salabert, D., et al. 2014, A\&A, 572, A34

Gray, D. F. 1984, ApJ, 281, 719

Gray, D. F. 2005, The Observation and Analysis of Stellar Photospheres (CUP)

Guinan, E. F., \& Engle, S. G. 2009, in The Ages of Stars, eds. E. E. Mamajek,

D. R. Soderblom, \& R. F. G. Wyse, IAU Symp., 258, 395

Gustafsson, B. 1998, Space Sci. Rev., 85, 419

Hekker, S., \& Meléndez, J. 2007, A\&A, 475, 1003

Howard, R., \& Harvey, J. 1970, Sol. Phys., 12, 23
Kawaler, S. D. 1988, ApJ, 333, 236

Lanzafame, A. C., \& Spada, F. 2015, A\&A, 584, A30

Leão, I. C., Pasquini, L., Ferreira Lopes, C. E., et al. 2015, A\&A, 582, A85

Magic, Z., Collet, R., Asplund, M., et al. 2013, A\&A, 557, A26

Maldonado, J., Martínez-Arnáiz, R. M., Eiroa, C., Montes, D., \& Montesinos, B. 2010, A\&A, 521, A12

Mamajek, E. E., \& Hillenbrand, L. A. 2008, ApJ, 687, 1264

Mason, B. D., Hartkopf, W. I., Holdenried, E. R., \& Rafferty, T. J. 2001, AJ, 121 3224

Matthews, C. T., Crepp, J. R., Skemer, A., et al. 2014, ApJ, 783, L25

Mayor, M., Pepe, F., Queloz, D., et al. 2003, The Messenger, 114, 20

Meléndez, J., Bergemann, M., Cohen, J. G., et al. 2012, A\&A, 543, A29

Melo, C. H. F., Pasquini, L., \& De Medeiros, J. R. 2001, A\&A, 375, 851

Noyes, R. W., Hartmann, L. W., Baliunas, S. L., Duncan, D. K., \& Vaughan, A. H. 1984, ApJ, 279, 763

Pace, G., \& Pasquini, L. 2004, A\&A, 426, 1021

Pinsonneault, M. H., Kawaler, S. D., Sofia, S., \& Demarque, P. 1989, ApJ, 338, 424

Pizzolato, N., Maggio, A., Micela, G., Sciortino, S., \& Ventura, P. 2003, A\&A, 397,147

Ramírez, I., Allende Prieto, C., \& Lambert, D. L. 2013, ApJ, 764, 78

Ramírez, I., Meléndez, J., Bean, J., et al. 2014, A\&A, 572, A48

Ribas, I., Guinan, E. F., Güdel, M., \& Audard, M. 2005, ApJ, 622, 680

Robles, J. A., Lineweaver, C. H., Grether, D., et al. 2008, ApJ, 684, 691

Saffe, C., Flores, M., \& Buccino, A. 2015, A\&A, 582, A17

Schirbel, L., Meléndez, J., Karakas, A. I., et al. 2015, A\&A, 584, A116

Skumanich, A. 1972, ApJ, 171, 565

Smith, M. A. 1979, PASP, 91, 737

Sneden, C. A. 1973, Ph.D. Thesis, The University of Texas at Austin

Soderblom, D. R. 1983, ApJS, 53, 1

Soderblom, D. R. 1985, AJ, 90, 2103

Soderblom, D. R., Duncan, D. K., \& Johnson, D. R. H. 1991, ApJ, 375, 722

Soderblom, D. R., Stauffer, J. R., Hudon, J. D., \& Jones, B. F. 1993, ApJS, 85 315

Sood, A., Kim, E.-j., \& Hollerbach, R. 2016, ApJ, submitted [arXiv: 1605.07125]

Strassmeier, K. G., Weber, M., Granzer, T., \& Järvinen, S. 2012, Astron. Nachr., 333,663

Talon, S., \& Zahn, J.-P. 1997, A\&A, 317, 749

Tokovinin, A. 2014a, AJ, 147, 86

Tokovinin, A. 2014b, AJ, 147, 87

Tucci Maia, M., Meléndez, J., Castro, M., et al. 2015, A\&A, 576, L10

Tucci Maia, M., Ramírez, I., Meléndez, J., et al. 2016, A\&A, 590, A32

van Saders, J. L., Ceillier, T., Metcalfe, T. S., et al. 2016, Nature, 529, 181

Yana Galarza, J., Meléndez, J., Ramírez, I., et al. 2016, A\&A, 589, A17

Yi, S., Demarque, P., Kim, Y.-C., et al. 2001, ApJS, 136, 417

Zahn, J.-P. 1992, A\&A, 265, 115 


\title{
Spectroscopic binaries in the Solar Twin Planet Search program: from substellar-mass to $M$ dwarf companions
}

\author{
Leonardo A. dos Santos, ${ }^{1 \star}$ Jorge Meléndez, ${ }^{1}$ Megan Bedell, ${ }^{2}$ Jacob L. Bean, ${ }^{2}$ \\ Lorenzo Spina, ${ }^{1}$ Alan Alves-Brito, ${ }^{3}$ Stefan Dreizler, ${ }^{4}$ Iván Ramírez, ${ }^{5}$ \\ and Martin Asplund 6 \\ ${ }^{1}$ Universidade de São Paulo, Instituto de Astronomia, Geofísica e Ciências Atmosféricas, Rua do Matão 1226, São Paulo \\ 05508-090, Brazil \\ ${ }^{2}$ University of Chicago, Department of Astronomy and Astrophysics, 5640 S. Ellis Ave, Chicago, IL 60637, USA \\ ${ }^{3}$ Universidade Federal do Rio Grande do Sul, Instituto de Física, Av. Bento Gonçalves 9500, Porto Alegre, RS, Brazil \\ ${ }^{4}$ University of Göttingen, Institut für Astrophysik, Germany \\ 5 Tacoma Community College, Washington, USA \\ ${ }^{6}$ The Australian National University, Research School of Astronomy and Astrophysics, Cotter Road, Weston, ACT 2611, Australia
}

Accepted XXX. Received YYY; in original form ZZZ

\begin{abstract}
Previous studies on the rotation of Sun-like stars revealed that the rotational rates of young stars converge towards a well-defined evolution that follows a power-law decay. It seems, however, that some binary stars do not obey this relation, often by displaying enhanced rotational rates and activity. In the Solar Twin Planet Search program, we observed several solar twin binaries, and found a multiplicity fraction of $42 \% \pm 6 \%$ in the whole sample; moreover, at least three of these binaries (HIP 19911, HIP 67620 and HIP 103983) clearly exhibit the aforementioned anomalies. We investigated the configuration of the binaries in the program, and discovered new companions for HIP 6407, HIP 54582, HIP 62039 and HIP 30037, of which the latter is orbited by a 0.06 $\mathrm{M}_{\odot}$ brown dwarf in a 1-month long orbit. We report the orbital parameters of the systems with well-sampled orbits and, in addition, the lower limits of parameters for the companions that only display a curvature in their radial velocities. For the linear trend binaries, we report an estimate of the masses of their companions when their observed separation is available, and a minimum mass otherwise. We conclude that solar twin binaries with low-mass stellar companions at moderate orbital periods do not display signs of a distinct rotational evolution when compared to single stars. We confirm that the three peculiar stars are double-lined binaries, and that their companions are polluting their spectra, which explains the observed anomalies.
\end{abstract}

Key words: stars: fundamental parameters - stars: solar-type - stars: rotation binaries: spectroscopic - binaries: visual

\section{INTRODUCTION}

It is known that at least half of the stars in the Galaxy are multiple systems containing two or more stars orbiting each other (Kiseleva-Eggleton \& Eggleton 2001; Fuhrmann et al. 2017), thus in many surveys and large samples of stars, binaries are ubiquitous. This is in contrast with the Sun, which is a single star, and attempts to find a faint stellar companion orbiting it rendered no results thus far (e.g., Luhman 2014). Many studies avoid contamination by binaries in their sam-

^ E-mail: leonardoags@usp.br ples, the main reasons being because we do not understand well how binaries evolve and how the presence of a companion affects the primary star. However, with the development of instruments with higher spatial and spectral resolution and coronagraphs, it is now possible to better probe the secondary component of such systems.

We have been carrying out a radial velocity planet search focused on solar twins using HARPS (Ramírez et al. 2014; Bedell et al. 2015; Tucci Maia et al. 2016; dos Santos et al. 2016; Meléndez et al. 2017, hereafter Papers I, II, III, IV and V, respectively). The definition of solar twin we use is a star with stellar parameters inside the ranges $5777 \pm 100$ 
$\mathrm{K}, 4.44 \pm 0.10 \mathrm{dex}(\mathrm{cgs})$ and $0.0 \pm 0.1 \mathrm{dex}$, respectively, for $T_{\text {eff }}, \log g$ and $[\mathrm{Fe} / \mathrm{H}]$. In total, 81 solar twins ${ }^{1}$ were observed on HARPS. As part of our survey we previously identified 16 clear spectroscopic binaries (SB) (Paper IV). We report here the identification of four additional SBs (HIP 14501, HIP 18844, HIP 65708 and HIP 83276) and the withdrawal of HIP 43297 and HIP 64673, which are unlikely to host stellar-mass companions, bringing the number of solar twin SBs to 18. Most of these SBs are single-lined - they do not contain a second component in their spectral lines -, meaning that their companions are either faint stars or located outside the $\sim 1^{\prime \prime}$ aperture of the HARPS spectrograph. We confirm that there are three solar twins with spectra contaminated by a relatively bright companion (see discussion in Section 4.3). In our sample there are an additional 18 visual binaries ${ }^{2}$ or multiple systems, of which HIP 6407 and HIP 18844 have wide companions (see table 5 in Tokovinin 2014) as well as the spectroscopic companions reported here.

In Paper IV we saw that the single or visual binary solar twins display a rotational evolution that can described with a relation between stellar age $t$ and rotational velocity $v_{\text {rot }}$ in the form of a power law plus a constant: $v_{\text {rot }}=v_{\mathrm{f}}+m t^{-b}$, where $v_{\mathrm{f}}, m$ and $b$ are free parameters to be fit with observations. This relation is explained by loss of angular momentum due to magnetized winds (e.g., Mestel 1984; Charbonneau 1992; Barnes 2003; Gallet \& Bouvier 2013), and the index $b$ reflects the geometry of the stellar magnetic field (Kawaler 1988). There are at least two solar twin binaries that display enhanced rotational velocities - above $2 \sigma$ from the expected - and activity for their ages: HIP 19911 and HIP 67620; if we consider the revised age for HIP 103983 (Spina et al., in preparation), it can also be considered a fast rotator for its age.

Besides the enhanced rotational velocities and higher chromospheric activity (Paper I; Freitas et al. 2017), some of these binaries also display peculiar chemical abundances (Paper III; Desidera et al. 2016). As pointed out by Desidera et al., the ultra-depletion of beryllium, which is observed on HIP 64150, could be explained by the interaction of the main star with the progenitor of the white dwarf companion. In addition to HIP 64150, the confirmed binaries HIP 19911 and HIP 67620 also display clearly enhanced [Y/Mg] abundances (see fig. 9 in Paper III, and the discussion in Section 4.3 of this paper).

One interesting aspect about stars with enhanced activity and rotation is that these characteristics were hypothesized to be the result of dynamo action from close-in giant planets (see Karoff et al. 2016, and references therein). In fact, some of our early results pointed out that the star HIP 68468 , for which we inferred two exoplanets candidates (Paper $\mathrm{V}$ ), had an enhanced rotational velocity when compared to other solar twins of the same age. However, a more careful analysis showed that the enhancement was instead a contribution of macroturbulence. Another explanation for these enhancements is due to magnetic interactions with either a

\footnotetext{
1 Some of the stars in our sample do not fit the strict definition of solar twins because one or more parameters are off the definition intervals, but they are still very close solar analogues.

2 We define as visual companions those with separations larger than $1^{\prime \prime}$
}

close-in or an eccentric giant planet (Cuntz et al. 2000), but recent results obtained by, e.g., Figueira et al. (2016) and Mengel et al. (2017) show that they cannot explain such anomalies.

In light of these intriguing results, we sought to better understand the nature of these solar twin multiple systems by studying their orbital parameters, and use them to search for explanations of the observed anomalies, especially stellar rotation. The orbital parameters can be estimated from the radial velocity data of the stars (see, e.g., Murray \& Correia 2010, hereafter $\mathrm{MC10}$ ), with the quality of the results depending strongly on the time coverage of the data.

\section{RADIAL VELOCITIES}

Our solar twins HARPS data ${ }^{3}$ are completely described in Paper I. Their radial velocities (RV) are automatically measured from the HARPS Data Reduction Software (see Table 1 ), and the noise limit of the instrument generally remains around $1 \mathrm{~m} \mathrm{~s}^{-1}$. In order to broaden the coverage of our RV data, we also obtained more datasets that were available in the literature and public databases, including the HARPS archival data for other programs.

The mass and other stellar parameters of the solar twins were estimated with high precision using the combined HARPS spectra and differential analysis owing to their similarity with the Sun (see, e.g., Bedell et al. 2014; Baumann et al. 2010; Yana Galarza et al. 2016). The ages for the solar twins were obtained using Yonsei-Yale isochrones (Yi et al. 2001) and probability distribution functions as described in Ramírez et al. (2013) and in Paper I. The full description and discussion of the stellar parameters of the HARPS sample are going to be presented in a forthcoming publication (Spina et al., in preparation).

The additional radial velocities data obtained from online databases and the literature are summarized in Table 2. These are necessary to increase the time span of the observations to include as many orbital phases as possible at the cost of additional parameters to optimize for (see Section $3.1)$.

\section{METHODS}

The variation of radial velocities of a star in binary or multiple system stems from the gravitational interaction between the observed star and its companions. For systems with stellar or substellar masses, the variation of radial velocities can be completely explained by the Keplerian laws of planetary motion. For the sake of consistency, we will use here the definitions of orbital parameters as presented in MC10.

To completely characterize the orbital motion of a binary system from the measured radial velocities of the main

\footnotetext{
3 Based on observations collected at the European Organisation for Astronomical Research in the Southern Hemisphere under ESO programs 188.C-0265, 183.D-0729, 292.C-5004, 077.C0364, 072.C-0488, 092.C-0721, 093.C-0409, 183.C-0972, 192.C0852, 091.C-0936, 089.C-0732, 091.C-0034, 076.C-0155, 185.D0056, 074.C-0364, 075.C-0332, 089.C-0415, 60.A-9036, 075.C0202, 192.C-0224, 090.C-0421 and 088.C-0323.
} 
Table 1. HARPS radial velocities for stars in the Solar Twin Planet Search program. This table is presented for guidance regarding the form and content of the online supplementary data, which is available in its entirety in machine-readable format.

\begin{tabular}{ccc}
\hline $\begin{array}{c}\text { Julian Date } \\
(\mathrm{d})\end{array}$ & $\begin{array}{c}\mathrm{RV} \\
\left(\mathrm{km} \mathrm{s}^{-1}\right)\end{array}$ & $\begin{array}{c}\sigma_{\mathrm{RV}} \\
\left(\mathrm{km} \mathrm{s}^{-1}\right)\end{array}$ \\
\hline \multicolumn{3}{c}{ HIP 6407} \\
2455846.750257 & 6.816873 & 0.001020 \\
2455850.716200 & 6.811186 & 0.000997 \\
2455851.710847 & 6.806352 & 0.000886 \\
2455852.703837 & 6.801079 & 0.001140 \\
2456164.849296 & 6.204053 & 0.001059 \\
2456165.853256 & 6.203359 & 0.001049 \\
2456298.564449 & 5.954045 & 0.001029 \\
\hline HIP 14501 \\
2452937.683821 & 7.024814 & 0.000475 \\
2452940.727854 & 7.025289 & 0.000516 \\
2453001.575609 & 7.026322 & 0.000658 \\
2453946.941856 & 7.023707 & 0.000708 \\
\hline
\end{tabular}

star, we need to obtain the following parameters: the semiamplitude of the radial velocities $K$, the orbital period $T$, the time of periastron passage $t_{0}$, the argument of periapse $\omega$ and the eccentricity $e$ of the orbit. In order to estimate the minimum mass $m \sin i$ of the companion and the semi-major axis $a$ of the orbit, we need to know the mass $M$ of the main star.

Due to their non-negative nature, the parameters $K$ and $T$ are usually estimated in logarithmic scale in order to eliminate the use of search bounds. Additionally, for orbits that are approximately circular, the value of $\omega$ may become poorly defined. In these cases, a change of parametrization may be necessary to better constrain them. Eastman et al. (2013), for instance, suggest using $\sqrt{e} \cos \omega$ and $\sqrt{e} \sin \omega$ (which we refer to as the EXOFAST parametrization) instead of $\omega$ and $e$ to circumvent this problem, which also can help improve convergence time.

One issue that affects the radial velocities method is the contamination by stellar activity (see, e.g., Haywood et al. 2016). This activity distorts the spectral lines (Gray 2005), which in turn produces artificial RV variations that can mimic the presence of a massive companion orbiting the star. More active stars are expected to have RV variations with larger amplitudes and a shorter activity cycle period (Lovis et al. 2011). For most binaries in our sample, the contamination by activity in the estimation of orbital parameters is negligible; the cases where this is not applicable are discussed in detail.

\subsection{Binaries with well-sampled orbits}

For binaries with orbital periods $T \lesssim 15 \mathrm{yr}$, usually there are enough RV data measured to observe a complete phase. In these cases, the natural logarithm of the likelihood of observing radial velocities $\mathbf{y}$ on a specific instrument, given the Julian dates $\mathbf{x}$ of the observations, their uncertainties $\sigma$ and the orbital parameters $\mathbf{p}_{\text {orb }}$ is defined as:

$\ln p\left(\mathbf{y} \mid \mathbf{x}, \sigma, \mathbf{p}_{\text {orb }}\right)=-\frac{1}{2} \sum_{n}\left[\frac{\left(y_{\mathrm{n}}-y_{\text {model }}\right)^{2}}{\sigma_{\mathrm{n}}^{2}}+\ln \left(2 \pi \sigma_{\mathrm{n}}^{2}\right)\right]$,

where $y_{\mathrm{n}}$ are the RV datapoints, $y_{\text {model }}$ are the model RV points for a given set of orbital parameters, and $\sigma_{\mathrm{n}}$ are the RV point-by-point uncertainties. The RV models are computed from Eq. 65 in MC10:

$v_{\mathrm{r}}=\gamma+K(\cos (\omega+f)+e \cos \omega)$,

where $f$ is the true anomaly, and $\gamma$ is the systemic velocity (usually including the instrumental offset). The true anomaly depends on $e$ and the eccentric anomaly $E$ :

$\cos f=\frac{\left(1-e^{2}\right)}{1-e \cos E}-1 ;$

the eccentric anomaly, in turn, depends on $T, t_{0}$ and time $t$ in the form of the so called Kepler's equation:

$E-e \sin E=\frac{2 \pi}{T}\left(t-t_{0}\right)$.

Eq. 1 is minimized using the Nelder-Mead algorithm implementation from Imfit (Newville et al. 2016, version 0.9.5) to obtain the best-fit orbital parameters to the observed data. Because different instruments have different instrumental offsets, the use of additional RV data from other programs require the estimation of an extra value of $\gamma$ for each instrument.

The uncertainties of the orbital parameters are estimated using emcee, an implementation of the Affine Invariant Markov chain Monte Carlo Ensemble sampler (ForemanMackey et al. 2013, version 2.2.1) using flat priors for all parameters in both MC10 and EXOFAST parametrizations. These routines were implemented in the Python package ra$\mathrm{dial}^{4}$, which is openly available online. The uncertainties in $m \sin i$ and $a$ quoted in our results already take into account the uncertainties in the stellar masses of the solar twins.

\subsection{Binaries with partial orbits}

For the binary systems with long periods (typically 20 years or more), it is possible that the time span of the observations does not allow for a full coverage of at least one phase of the orbital motion. In these cases, the estimation of the orbital parameters renders a number of possible solutions, which precludes us from firmly constraining the configuration of the system. Nevertheless, RV data containing a curvature or one inflection allows us to place lower limits on $K, T$ and $m \sin i$, whilst leaving $e$ and $\omega$ completely unconstrained. When the RV data are limited but comprise two inflections, it may be possible to use the methods from Section 3.1 to constrain the orbital parameters, albeit with large uncertainties.

For stars with very large orbital periods $(T \gtrsim 100 \mathrm{yr})$, the variation of radial velocities may be present in the form

\footnotetext{
${ }^{4}$ Available at https://github.com/RogueAstro/radial.
} 
Table 2. Additional radial velocities from other programs and instruments.

\begin{tabular}{lll}
\hline Instrument/Program & References & Data available for (HIP numbers) \\
\hline CfA Digital Speedometers & Latham et al. (2002) & 65708 \\
ELODIE $^{\text {a }}$ & Baranne et al. (1996); Moultaka et al. (2004) & $43297,54582,62039,64150,72043,87769$ \\
SOPHIE $^{\mathrm{b}}$ & Perruchot et al. (2011) & $6407,43297,54582,62039,64150,87769$ \\
Lick Planet Search & Fischer et al. (2014) & 54582,65708 \\
AAT Planet Search & Jenkins et al. (2015) & $18844,67620,73241,79578,81746$ \\
Various & Willmarth et al. (2016) & 67620 \\
HIRES/Keck RV Surveyc & Butler et al. (2017) & $14501,19911,62039,64150,72043,103983$ \\
\hline${ }^{a}$ Available at http://atlas.obs-hp.fr/elodie/. & \\
${ }^{b}$ Available at http://www.obs-hp.fr/guide/sophie/data_products.shtml. & \\
${ }^{c}$ Available at http://home.dtm.ciw.edu/ebps/data/. &
\end{tabular}

of a simple linear trend. In these cases, it is still possible to obtain an estimate of the mass of the companion - a valuable piece of information about it: Torres (1999) describes a statistical approach to extract the sub-stellar companion mass when the only information available from radial velocities is the inclination of the linear trend, provided information about the angular separation of the system is also available. In this approach, we need to adopt reasonable prior probability density functions (PDF) for the eccentricity $e$, the longitude of periastron $\varpi$, phase $\phi$ and the inclination $i$ of the orbital plane. As in Torres, we adopt the following PDFs: $p(i)=\sin i, p(e)=2 e$ and flat distributions for $\varpi$ and $\phi$.

We sample the PDFs using emcee, 20 walkers and 10000 steps; the first 500 burn-in steps are discarded. From these samples, we compute the corresponding companion masses and its posterior distribution. This posterior usually displays a very strong peak and long tails towards low and high masses which can be attributed to highly unlikely orbital parameters (see Fig. 6 for an example). In our results, we consider that the best estimates for the companion masses are the central bin of the highest peak of the distribution in a histogram with log-space bin widths of about 0.145 $\operatorname{dex}\left(\mathrm{M}_{\odot}\right)$.

When no AO imaging data are available for the stars with a linear trend in their RVs, the most conservative approach is to provide the minimum mass for the putative companion. In the case of a linear trend, the lowest mass is produced when $e=0.5, \omega=\pi / 2$ and $\sin i=1$ (Feng et al. 2015), yielding

$m_{\min } \approx\left(0.0164 \mathrm{M}_{\mathrm{Jup}}\right)\left(\frac{\tau}{\mathrm{yr}}\right)^{4 / 3}\left|\frac{d v / d t}{\mathrm{~m} \mathrm{~s}^{-1} \mathrm{yr}^{-1}}\right|\left(\frac{M}{\mathrm{M}_{\odot}}\right)^{2 / 3}$,

where $\tau$ is 1.25 multiplied by the time span of the radial velocities and $d v / d t$ is the inclination of the linear trend.

\section{RESULTS}

We discovered new, short-period companions for the stars HIP 6407 and HIP 30037 (see Fig. 1) and new long-period companions for HIP 54582 and HIP 62039, and updated or reproduced the parameters of several other known binaries that were observed in our program (see Figs. 2 and 4). We briefly discuss below each star, pointing out the most interesting results, inconsistencies and questions that are still open about each of them. The orbital parameters of the binaries with well-sampled orbits in their RV data are presented in Table 3 and the systems with partial orbits are reported in Tables 4 and 5 .

\subsection{Withdrawn binary candidates}

In Paper IV, we showed that HIP 43297 had a rotational velocity $v \sin i$ higher than expected for its age. Moreover, its radial velocities had variations that hinted for one or more companions orbiting it. We carefully analyzed the RVs and concluded that the periodic $(T=3.8 \mathrm{yr})$ signal observed is highly correlated (Pearson $R=0.893$ ) with the activity $S$-index of the star (Freitas et al. 2017). In addition, we tentatively fitted a linear trend to the combined RVs from HARPS, ELODIE and SOPHIE, and obtained an inclination of $4.53 \pm 0.04 \mathrm{~m} \mathrm{~s}^{-1} \mathrm{yr}^{-1}$, but further monitoring of the system is required to infer the presence of a long-period spectroscopic companion. The revised stellar age for HIP 43297 yields $1.85 \pm 0.50$ Gyr (Spina et al., in preparation), which explains the high rotational velocity and activity.

The solar twin HIP 64673 displays significant fluctuations in its radial velocities, but they do not correlate with its activity index; the data covers approximately 5 years of $\mathrm{RV}$ monitoring and displays an amplitude $>20 \mathrm{~m} \mathrm{~s}^{-1}$. If confirmed to be caused by massive companions, the RV variations of both HIP 43297 and HIP 64673 suggest substellar masses for the most likely orbital configurations. These stars are, thus, removed from the binaries sample of the Solar Twin Planet Search program.

\subsection{Solar twins with new companions}

HIP 6407: This is a known binary system located 58 pc away from the solar system (van Leeuwen 2007), possessing a very low-mass $\left(0.073 \mathrm{M}_{\odot}\right)$ L2-type companion separated by $44.8^{\prime \prime}$ (2222 AU), as reported by Baron et al. (2015, and references therein). In this study, we report the detection of a new close-in low-mass companion with $m \sin i=0.12 \mathrm{M}_{\odot}$ on a very eccentric orbit $(e=0.67)$ with $a=3 \mathrm{AU}$ and an orbital period of approximately 5 years. As expected, the long-period companion does not appear in the RV data as a linear trend.

HIP 30037: The most compact binary system in our sample, hosting a brown dwarf companion orbiting the main 

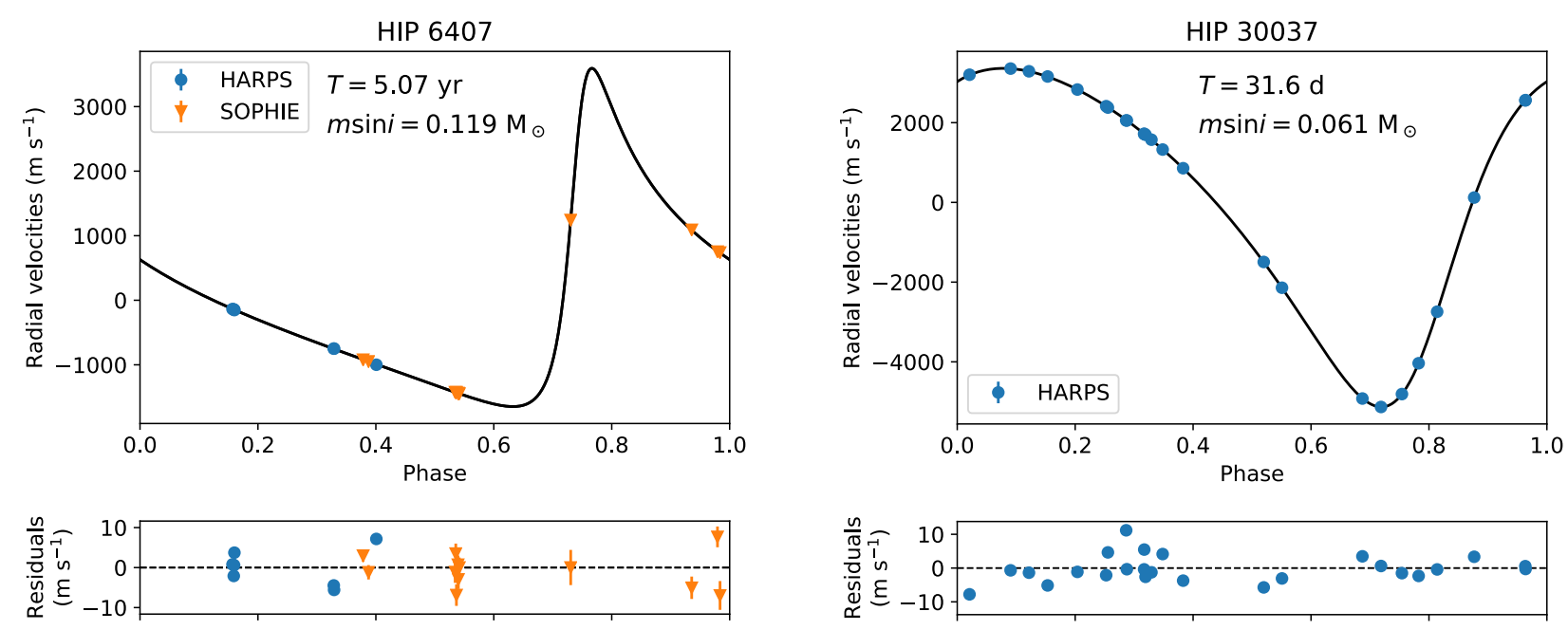

Figure 1. The radial velocities and orbital solutions of the newly discovered companions for solar twins with short orbital periods.

Table 3. Orbital parameters of the binaries with complete or near-complete orbital phases in their RV data.

\begin{tabular}{|c|c|c|c|c|c|c|c|c|}
\hline HIP & HD & $\begin{array}{c}K \\
\left(\mathrm{~km} \mathrm{~s}^{-1}\right)\end{array}$ & $\begin{array}{c}T \\
\text { (days) }\end{array}$ & $\begin{array}{c}t_{0} \\
\text { (JD-2.45E6 days) }\end{array}$ & $\begin{array}{c}\omega \\
\left({ }^{o}\right)\end{array}$ & $e$ & $\begin{array}{c}m \sin i \\
\left(\mathrm{M}_{\odot}\right)\end{array}$ & $\begin{array}{c}a \\
(\mathrm{AU})\end{array}$ \\
\hline \multirow{2}{*}{$6407^{\dagger}$} & \multirow{2}{*}{8291} & 2.614 & 1852.3 & 5076.7 & -57.1 & 0.682 & 0.119 & 3.070 \\
\hline & & \pm 0.084 & $\begin{array}{r}+3.3 \\
-3.1\end{array}$ & $\begin{array}{l}+1.1 \\
-1.3\end{array}$ & \pm 0.9 & $\begin{array}{l}+0.009 \\
-0.010\end{array}$ & \pm 0.002 & \pm 0.005 \\
\hline \multirow{2}{*}{19911} & \multirow{2}{*}{26990} & 7.707 & 2074.15 & 4627.4 & 40.92 & 0.8188 & 0.313 & 6.16 \\
\hline & & \pm 0.007 & \pm 0.09 & \pm 0.1 & \pm 0.06 & \pm 0.0003 & \pm 0.002 & \pm 0.02 \\
\hline \multirow{2}{*}{30037} & \multirow{2}{*}{45021} & 4.246 & 31.61112 & 5999.413 & -133.60 & 0.30205 & 0.0610 & 0.1971 \\
\hline & & \pm 0.003 & \pm 0.00006 & \pm 0.001 & \pm 0.02 & \pm 0.00008 & \pm 0.0002 & \pm 0.0003 \\
\hline \multirow{2}{*}{65708} & \multirow{2}{*}{117126} & 5.754 & 207.273 & -3675.8 & -137.9 & 0.311 & 0.170 & 0.851 \\
\hline & & \pm 0.007 & \pm 0.004 & \pm 0.3 & \pm 0.2 & \pm 0.002 & \pm 0.001 & \pm 0.001 \\
\hline \multirow{2}{*}{67620} & \multirow{2}{*}{120690} & 6.311 & 3803.3 & 4945.7 & 145.10 & 0.3428 & 0.578 & 5.50 \\
\hline & & \pm 0.002 & \pm 0.4 & \pm 0.7 & \pm 0.07 & \pm 0.0002 & \pm 0.002 & \pm 0.01 \\
\hline \multirow{2}{*}{79578} & \multirow{2}{*}{145825} & 1.125 & 6681.8 & 879.2 & 138.9 & 0.3322 & 0.1014 & 7.216 \\
\hline & & \pm 0.007 & \pm 1.5 & \pm 1.9 & \pm 0.1 & \pm 0.0003 & \pm 0.0002 & \pm 0.007 \\
\hline \multirow{2}{*}{81746} & \multirow{2}{*}{150248} & 1.987 & 3246.5 & 5623.8 & -2.49 & 0.6644 & 0.1079 & 4.387 \\
\hline & & \pm 0.001 & \pm 0.7 & \pm 0.6 & \pm 0.06 & \pm 0.0005 & \pm 0.0002 & \pm 0.003 \\
\hline \multirow{2}{*}{103983} & \multirow{2}{*}{200565} & 2.100 & 10278 & 6659 & -51.6 & 0.50 & 0.210 & 9.8 \\
\hline & & \pm 0.018 & $\begin{array}{l}+274 \\
-247\end{array}$ & \pm 11 & \pm 1.5 & \pm 0.01 & \pm 0.005 & \pm 0.2 \\
\hline
\end{tabular}

$\dagger$ Triple or higher-order system. The orbital parameters correspond to the closer-in companion.

star with a period of 31 days. The high precision of its parameters owes to the wide time span of observations, which covered several orbits. This is one of the first detections of a close-in brown dwarf orbiting a confirmed solar twin ${ }^{5}$. HIP 30037 is a very quiet star, displaying no excessive jitter noise in its radial velocities. We ran stellar evolution models with MESA $^{6}$ (Paxton et al. 2011, 2015) to test the hypothesis of the influence of tidal acceleration caused by the companion on a tight orbit, and found that, for the mass and period of the companion, we should expect no influence in the rotational velocity.

HIP 54582: $R V$ Curvature only. There are no reports of binarity in the literature. The slight curvature in the RVs

\footnotetext{
5 There are at least 4 solar twin candidates with a close-in brown dwarf companion listed in table A.1 in Wilson et al. (2016).

6 Modules for Experiments in Stellar Astrophysics, available at http://mesa. sourceforge.net
}

of this star is only visible when we combine the HARPS data and the Lick Planet Search archival data. Owing to the absence of an inflection point, the orbital parameters of this system are highly unconstrained. We found that an orbit with $e \approx 0.2$ produces the least massive companion and shortest orbital period $\left(m \sin i=0.03 \mathrm{M}_{\odot}\right.$ and $\left.T=102 \mathrm{yr}\right)$.

HIP 62039: Linear trend. There are no reports of visually detected close-in $\left(\rho<2^{\prime \prime}\right)$ companions around it. This can be attributed to: i) low luminosity companion, which is possible if it is a white dwarf or a giant planet, and ii) unfavorable longitude of periapse during the observation windows. By using Eq. 5, we estimate that the minimum mass of the companion is $19 \mathrm{M}_{\mathrm{Jup}}$. 

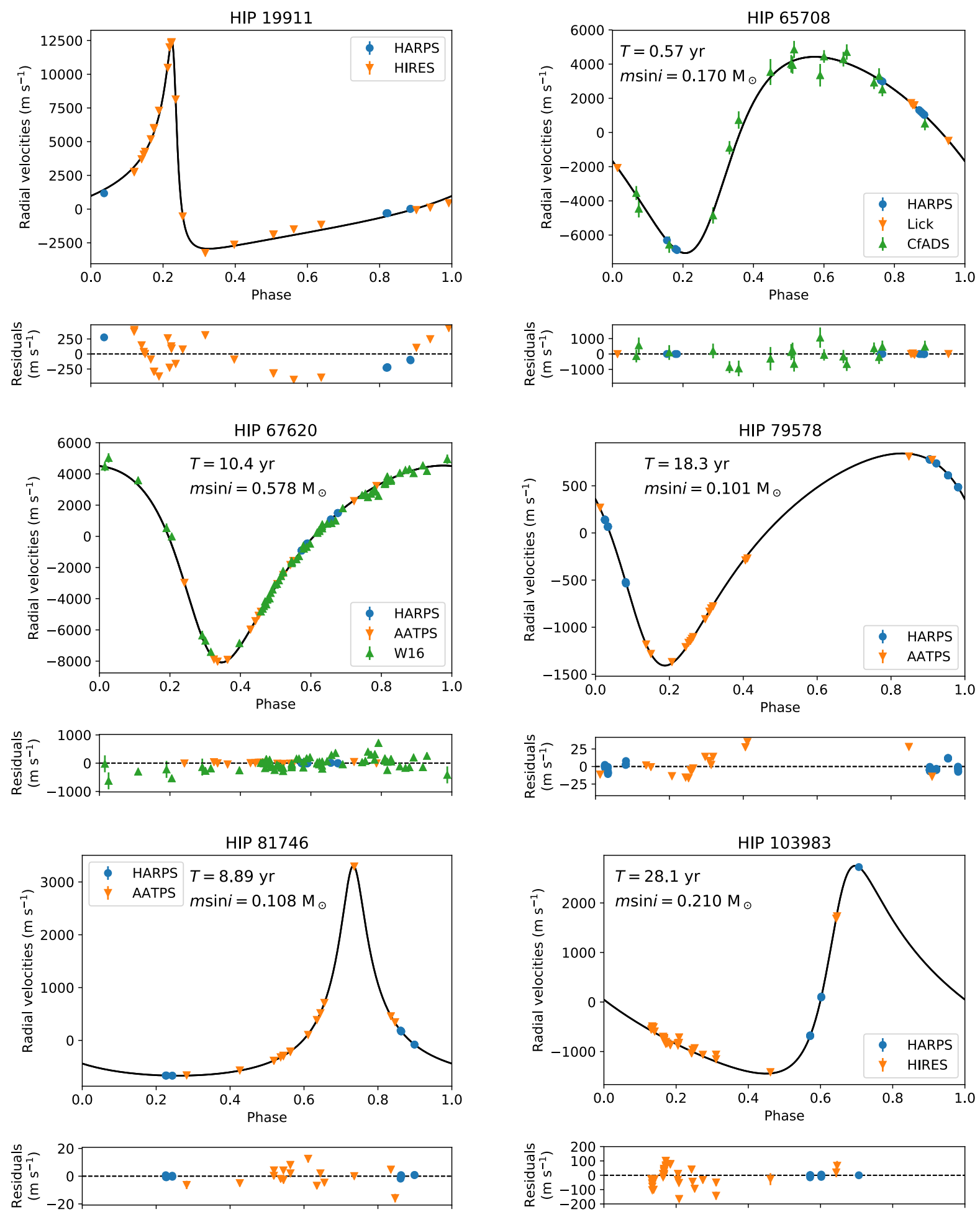

Figure 2. The radial velocities and updated orbital solutions of the known solar twin binaries with at least one complete or nearcomplete orbital phase. The acronyms CfADS, W16 and AATPS correspond to data from, respectively, the Center for Astrophysics Digital Speedometers, Willmarth et al. (2016) and the Anglo-Australian Telescope Planet Search program. 


\subsection{The peculiar binaries}

\subsubsection{HIP 19911}

This is one of the main outlier stars in the overall sample of solar twins in regards to its rotation and activity, which are visibly enhanced for both the previous and revised ages (Paper IV; Freitas et al. 2017; Spina et al., in preparation). For the estimation of orbital parameters reported below, we used only the LCES HIRES/Keck radial velocities, because there are too few HARPS data points to justify the introduction of an extra source of uncertainties (the HARPS points are, however, plotted in Fig. 2 for reference). When using the HARPS data, although the solution changes slightly, our conclusions about the system remain the same.

The orbital solution of HIP 19911 renders a $0.31 \mathrm{M}_{\odot}$ companion in a highly eccentric orbit $(e=0.82$, the highest in our sample), with period $T=5.7 \mathrm{yr}$. Visual scrutiny reveals what seems to be another signal with large amplitude in the residuals of this fit $\left(>250 \mathrm{~m} \mathrm{~s}^{-1}\right.$, see Fig. 2); the periodogram of the residuals shows a very clear peak near the orbital period of the stellar companion.

The cross-correlation function (CCF) plots for the HARPS spectra of HIP 19911 display a significant asymmetry - longer tail in the blue side - for the observations between October 2011 and February 2012, which suggests that the companion is contaminating the spectra. Upon visual inspection of the archival HIRES spectra ${ }^{7}$ taken on 17 January 2014, which is when we expect the largest RV difference between the main star and its companion, we saw a clear contamination of the spectrum by the companion (see Fig. 3). This contamination could explain the large residuals of the orbital solution, as it introduces noise to the measured radial velocities. The double-lines also explain the inferred high rotational velocity of HIP 19911, since they introduce extra broadening to the spectral lines used to measure rotation. The presence of a bright companion may also affect estimates of chemical abundances, which elucidates the yttrium abundance anomaly (Paper III). The double-lined nature of this system is not observed on the HARPS spectra due to an unfavorable observation window.

Even at the largest RV separation, we did not detect the Li I line at $6707.75 \AA$ in the HIRES spectrum of the companion. This is expected because M dwarf stars have deeper convection zones, which means they deplete lithium much faster than Sun-like stars. This leads us to conclude that estimates of Li abundance on solar twin binaries using this line do not suffer from strong contamination by their companions; consequently, age estimates with lithium abundances may be more reliable for such binaries than isochronal or gyro ages.

Another observation conundrum for this system is that Riddle et al. (2015), using AO imaging without a coronagraph, reports the detection of a visual companion with orbital period $\sim 12.4 \mathrm{yr}$ (roughly twice the one we estimated), a lower eccentricity $(e=0.1677)$ and similar semi-major axis of the orbit ( $a=6.17 \mathrm{AU}$, if we consider a distance of 30.6 pc). Moreover, Tokovinin (2014, hereafter T14) reports that this visual companion has $m=0.85 \mathrm{M}_{\odot}$. The most likely explanation is that the observations of Riddle et al. did in fact

7 Available at http://nexsci.caltech.edu/archives/koa/. detect the spectroscopic companion, but the coarse timing of the observations produced a larger period; the lower eccentricity could be explained by a strong covariance between $e$ and the inclination $i$. If $i$ is lower, that means the mass of the companion is significantly higher than $m \sin i=0.316$ $\mathrm{M}_{\odot}$, and that would explain the value obtained by T14. A companion with a mass as large as $0.85 \mathrm{M}_{\odot}$ would likely pollute the spectra of HIP 19911, which agrees with our observation that this is a SB II system. If confirmed, this prominent $\sim 0.85 \mathrm{M}_{\odot}$ red dwarf companion could explain the observed activity levels for HIP 19911, since red dwarf stars are expected to be more active than Sun-like stars.

\subsubsection{HIP 67620}

This is a well-known binary and the target with the largest amount of RV data available (see Fig. 2). Its orbital parameters have been previously determined by Abt \& Willmarth (2006) and more recently by Jenkins et al. (2015) and Willmarth et al. (2016). The orbital parameters we obtained are in good agreement with Willmarth et al. (2016). It has one of the most peculiar rotation rates from our sample $(2.77$ $\mathrm{km} \mathrm{s}^{-1}$ for an age of $7.18 \mathrm{Gyr}$ ), an enhanced chromospheric activity (Freitas et al. 2017) and an anomalous [Y/Mg] abundance (Paper III). The orbital period of the system is far too long for gravitational interaction to enhance the rotation of the main star through tidal acceleration, thus we should expect that they evolve similarly to single stars from this point of view.

High-resolution imaging of HIP 67620 revealed a companion with $V_{\text {mag }} \approx 10$ and separations which are consistent with the spectroscopic companion (Hartkopf et al. 2012; T14). As explained by Jenkins et al. (2015), the presence of a companion with $m>0.55 \mathrm{M}_{\odot}$ can produce contaminations to the spectra that introduce noise to the measured RVs; our estimate of $m \sin i$ for this system is $0.58 \mathrm{M}_{\odot}$. These results suggest that, similarly to HIP 19911 but to a lesser degree, the companion of HIP 67620 may be offsetting our estimates for rotational velocity, stellar activity, chemical abundances and isochronal age.

We were unable to discern double-lines in the HARPS spectra, likely resulting from unfavorable Doppler separations (observations range from February 2012 to March 2013). However, an analysis of the CCF of this star shows slight asymmetries in the line profiles of the HARPS spectra, which indicates a possible contamination by the companion. Fuhrmann et al. (2017) reported HIP 67620 as a double-lined binary using spectra taken at high-resolution $(R \approx 60,000)$ in February 2014 and July 2015. As expected due to the short time coverage of the HARPS spectra, we did not see any correlation between the bisector inverse slope (as defined in Queloz et al. 2001) and the radial velocities of HIP 67620 .

Jenkins et al. (2015) found an additional signal on the periodogram of HIP 67620 at $532 \mathrm{~d}$, which could be fit with a $1 \mathrm{M}_{\text {Jup }}$ planet, bringing down the $r m s$ of the fit by a factor of 2. However, we did not find any significant peak in the periodogram of the residuals of the radial velocities for HIP 67620 . 

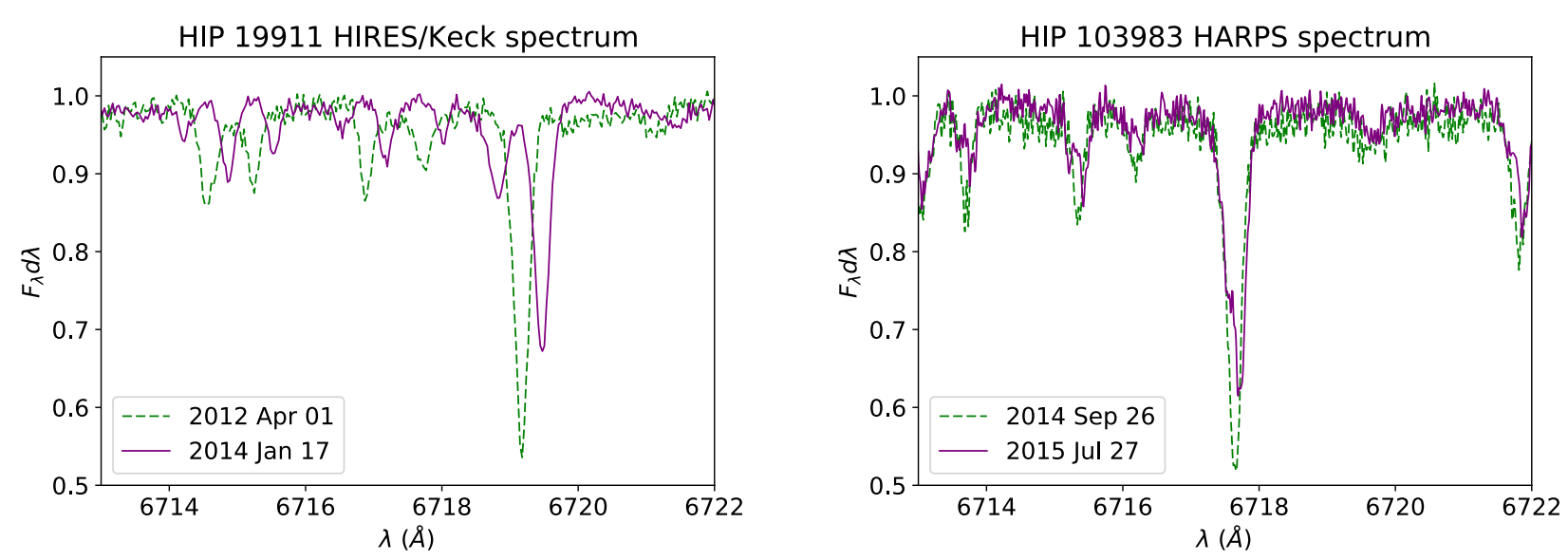

Figure 3. The spectrum of HIP 19911 and HIP 103983 are contaminated by bright companions. The double-lined nature of these stars is only clear only in the maximum RV separation, which are the solid purple curves. The dashed green curves show spectra during minimum RV separation where there is no obvious contamination. The spectra in this figure are not Doppler-corrected for their systemic velocities or instrumental RV offset.

\subsubsection{HIP 103983}

The revised age for HIP 103983 (4.9 \pm 0.9 Gyr; Spina et al., in preparation) renders this system as an abnormally fast rotator $\left(3.38 \mathrm{~km} \mathrm{~s}^{-1}\right)$ for its age. However, upon a careful inspection of the HARPS data obtained at different dates, we identified that the spectrum from 2015 July 27 displays clearly visible double-lines, albeit not as well separated as those observed in the HIRES spectra of HIP 19911 (see Fig. 3). No other anomalies besides enhanced rotation were inferred for this system. The CCF plots of the HARPS spectra show clear longer tails towards the blue side for most observations.

In Paper IV we reported distortions in the combined spectra of HIP 103983; this is likely a result from the combination of the spectra at orbital phases in which the Doppler separation between the binaries is large. Since the observing windows of the HARPS spectra of HIP 19911 and HIP 67620 do not cover large RV separations (see Fig. 2), the same effect is not seen in the combined spectra of these stars. This effect also explains why HIP 103983 is an outlier in fig. 4 of Paper IV.

Although we have limited RV data, the emcee simulations converge towards a well-defined solution instead of allowing longer periods, as these produce larger residuals. It is important, however, to keep monitoring the radial velocities of this system in order to confirm that the most recent data points are in fact a second inflection in the radial velocities. The residuals for the fit for the HIRES spectra are on the order of $100 \mathrm{~m} \mathrm{~s}^{-1}$, which is likely a result from the contamination by a bright companion. T14 reported a $0.91 \mathrm{M}_{\odot}$ visual companion at a separation of $0.093^{\prime \prime}$, which is consistent with the spectrocopic semi-major axis we estimated: $0.149^{\prime \prime}$ for a distance of 65.7 pc (van Leeuwen 2007).

\subsection{Other binaries with updated orbital parameters}

Among the known binaries in the solar twins sample, five of them display curvature in their RV data which allows the estimation of limits for their orbital parameters (see Table 4 and Fig. 4). Some of the linear trend binaries observed in our HARPS Solar Twin Planet Search program are targets with large potential for follow-up studies. For the companions with visual detection, we were able to estimate their most likely mass (see Table 5).

HIP 14501: Linear trend. Its companion is reported by Crepp et al. (2014) as the first directly imaged T dwarf that produces a measurable doppler acceleration in the primary star. Using a low-resolution direct spectrum of the companion, Crepp et al. (2015) estimated a model-dependent mass of $56.7 \mathrm{M}_{\text {Jup }}$. Using the HARPS and HIRES/Keck RV data and the observed separation of 1.653" (Crepp et al. 2014), we found that the most likely value of the companion mass is $0.043 \mathrm{M}_{\odot}\left(45 \mathrm{M}_{\text {Jup }}\right)$, which agrees with the mass obtained by Crepp et al. (2015). The most recent HARPS data hints of an inflection point in the orbit of HIP 14501 B (see Fig. 5 ), but further RV monitoring of the system is necessary to confirm it.

HIP 18844: Linear trend. It is listed as a multiple system containing a closer-in low-mass stellar companion (estimated $0.06 \mathrm{M} \odot$, which agrees with our most likely mass) and orbital period $T=6.5 \mathrm{yr}$ (T14, and references therein). For the companion farther away, Jenkins et al. (2015) reported a minimum orbital period of $\sim 195 \mathrm{yr}$ and $m \sin i=0.33 \mathrm{M}_{\odot}$, with a separation of $29^{\prime \prime}$ in $1941(\sim 750$ AU for a distance of $26 \mathrm{pc})$.

HIP 54102: $R V$ curvature only. It is listed as a proper motion binary by Makarov \& Kaplan (2005), but there are no other information about the companions in the literature. Its eccentricity is completely unconstrained due to lack of RV coverage. We estimate that its companion's minimum mass is $12.6 \mathrm{M}_{\mathrm{Jup}}$, with an orbital period larger than 14 years.

HIP 64150: Linear trend. The most likely companion mass obtained by the method explained in Section 3.2 renders an estimate of $0.26 \mathrm{M}_{\odot}$, as seen in Fig. 6. The higher mass $\left(0.54 \mathrm{M}_{\odot}\right)$ obtained by Crepp et al. 2013 and M14 can be attributed to less likely orbital configurations, but it is still inside the $1-\sigma$ confidence interval of the RV+imaging 

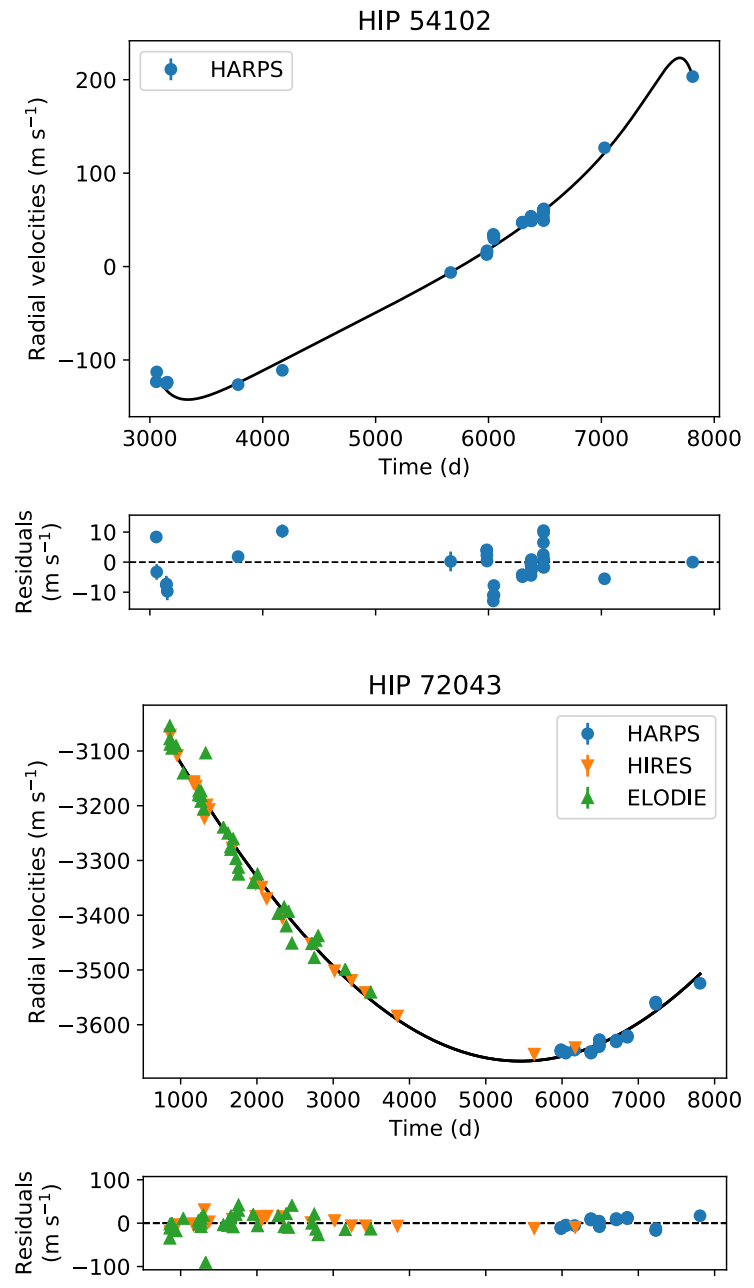
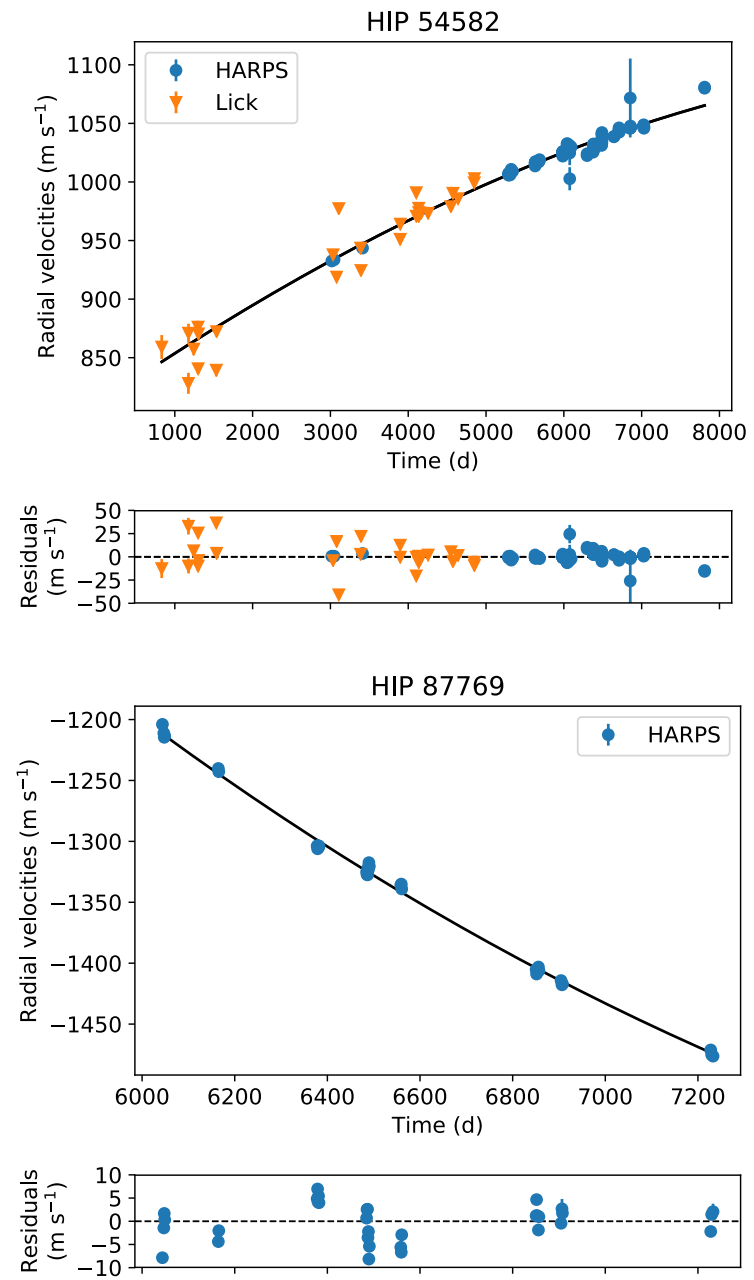

Figure 4. The radial velocities and the solutions that produced the lower limits of orbital parameters of the solar twin binaries with RV curvature. A similar plot for HIP 73241 can be found in Jenkins et al. (2015). Time is given in JD $-2.45 \times 10^{6} \mathrm{~d}$.

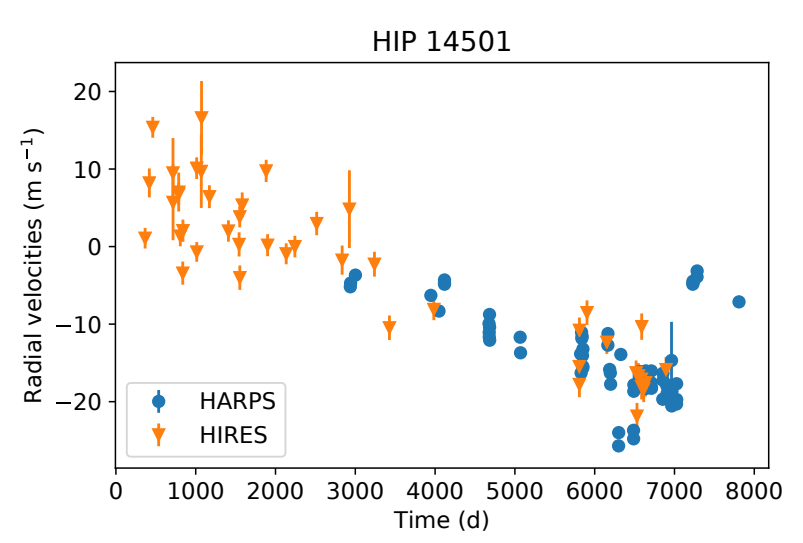

Figure 5. Radial velocities of HIP 14501. The RV shift in the $y$-axes is arbitrary. Time is given in $\mathrm{JD}-2.45 \times 10^{6} \mathrm{~d}$.

mass estimate. The main star displays clear signals of atmosphere pollution caused by mass transfer from its companion during the red giant phase (Takeda et al. 2011), characterizing the only confirmed blue straggler of our sample. The measured projected separation of the binary system is 18.1 AU (M14), which indicates that even for such a wide system the amount of mass transferred is still large enough to produce measurable differences in chemical abundances. It seems, however, that the amount of angular momentum transfer was not enough to produce significant enhancement in the rotation rate and activity of the solar twin. It is also important to note that the isochronal age measured for this system (Paper III) has a better agreement with the white dwarf (WD) cooling age estimated by M14 than previous estimates, illustrating the importance of studying these Siriuslike systems to test the various methods of age estimation.

HIP 65708: This star has previously been reported as a single-lined spectroscopic binary with an orbital solution (Latham et al. 2002). Here we update this solution by leveraging the extremely precise radial velocities measured in the Lick Planet Search program and with the HARPS spectrograph. The minimum mass of the companion is $0.167 \mathrm{M}_{\odot}$, indicating it is a red dwarf, orbiting at less than $1 \mathrm{AU}$ with a slight eccentricity of 0.31 . Our results agree with the previous orbital solution, which was based solely on data with uncertainties two orders of magnitude higher than the most recent data from HARPS and the Lick Planet Search. 


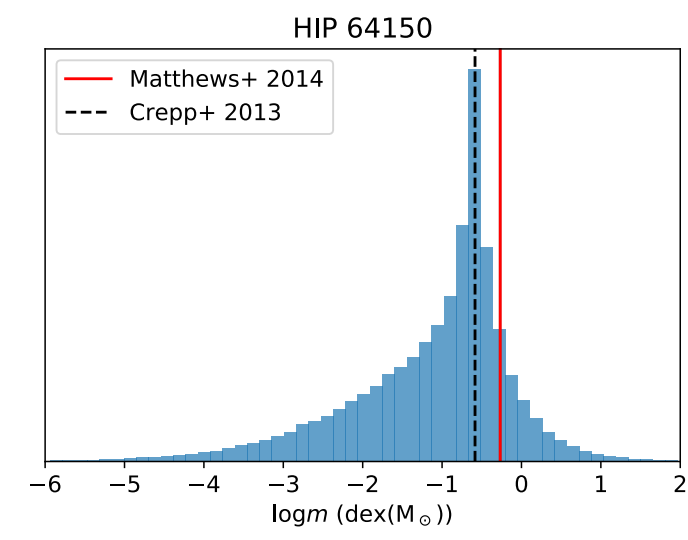

Figure 6. Posterior probability distribution of the companion mass for HIP 64150. The mass obtained by Matthews et al. (2014) using SED fitting for the spectra of the WD companion is shown as a red vertical line.

HIP 72043: $R V$ curvature only. Similarly to HIP 54102 , it is listed as a proper motion binary and we could not constrain its eccentricity. A fairly massive $\left(>0.5 \mathrm{M}_{\odot}\right)$ companion is inferred at a very large period; this fit suggests that the longitude of periapse of the companion of HIP 72043 is currently at an unfavorable position for visual detection.

HIP 73241: $R V$ curvature only. The companion's orbit is eccentric enough to allow an estimation of the minimum eccentricity; its companion has been previously been confirmed by Raghavan et al. (2010) and visually detected by T14 with a separation $0.318^{\prime \prime}$. In Paper IV we listed this star as having an unusually high rotation, but here we revise this conclusion and list HIP 73241 as a candidate peculiar rotator because its $v \sin i$ is less than $2 \sigma$ above the expected value for its age. Similarly to HIP 67620 , this peculiarity, if real, could also be explained by contamination by a bright companion, since we determined that the minimum companion mass $m \sin i>0.49 \mathrm{M}_{\odot}$

HIP 79578: The companion is a well-defined $0.10 \mathrm{M}_{\odot}$ red dwarf orbiting the main star approximately every 18 years in a fairly eccentric orbit $(e=0.33)$. The orbital parameters we obtained differ significantly from the ones obtained by Jenkins et al. (2015) by more than 10\%, except for the eccentricity; also in contrast, Jenkins et al. report it as a brown dwarf companion. The fit for this binary displays residuals of up to $30 \mathrm{~m} \mathrm{~s}^{-1}$ for the AATPS radial velocities, and the periodogram of these residuals shows a peak near the period 725 days. When we fit an extra object with $m \sin i=0.70 \mathrm{M}_{\mathrm{Jup}}$ at this period $(a=1.62 \mathrm{AU}$ and $e=0.87)$, it improves the general fit of the RVs by a factor of 7 . It is important to mention, however, that there are only 17 data points for the AATPS dataset, and the HARPS dataset does not display large residuals for a single companion fit. We need thus more observations to securely infer the configuration of this binary system, and if it truly has an extra substellar companion at a shorter period.

HIP 81746: This is another high-eccentricity $(e=0.7)$ binary that does not display clear anomalies in its rotation and activity. Its companion is a $0.1 \mathrm{M}_{\odot}$ red dwarf orbiting the main star every 9 years. The orbital parameters we obtained are in good agreement with the ones reported by Jenkins et al. (2015).
Table 4. Lower limits of the orbital parameters of the spectroscopic binaries with curvature in their RV data.

\begin{tabular}{llcccc}
\hline HIP & HD & $\begin{array}{c}K \\
\left(\mathrm{~km} \mathrm{~s}^{-1}\right)\end{array}$ & $\begin{array}{c}T \\
(\mathrm{yr})\end{array}$ & $\begin{array}{c}m \sin i \\
\left(\mathrm{M}_{\odot}\right)\end{array}$ & $e$ \\
\hline 54102 & 96116 & $>0.182$ & $>14$ & $>0.012$ & $\ldots$ \\
54582 & 97037 & $>0.193$ & $>102$ & $>0.03$ & $\ldots$ \\
72043 & 129814 & $>2.11$ & $>104$ & $>0.40$ & $\ldots$ \\
73241 & 131923 & $>5.93$ & $>21.0$ & $>0.49$ & $>0.72$ \\
87769 & 163441 & $>1.90$ & $>81.5$ & $>0.30$ & $\ldots$ \\
\hline
\end{tabular}

Table 5. Measured RV slopes of the linear trend binaries. The most likely mass for the spectroscopic companion is estimated when their separation is available. Otherwise a minimum mass is provided.

\begin{tabular}{llcccc}
\hline HIP & HD & $\begin{array}{c}d v_{r} / d t \\
\left(\mathrm{~m} \mathrm{~s}^{-1} \mathrm{yr}^{-1}\right)\end{array}$ & $\begin{array}{c}\rho \\
(\operatorname{arcsec})\end{array}$ & $\begin{array}{c}\text { Dist. } \\
(\mathrm{pc})^{\mathrm{d}}\end{array}$ & $\begin{array}{c}m \\
\left(\mathrm{M}_{\text {Jup }}\right)\end{array}$ \\
\hline 14501 & 19467 & $-1.30 \pm 0.01$ & $1.653^{\mathrm{a}}$ & 30.86 & 45 \\
$18844^{\dagger}$ & 25874 & $424 \pm 3$ & $0.140^{\mathrm{b}}$ & 25.91 & 79 \\
62039 & 110537 & $7.25 \pm 0.03$ & $\ldots$ & 42.68 & $>19$ \\
64150 & 114174 & $61.72 \pm 0.02$ & $0.675^{\mathrm{c}}$ & 26.14 & 270 \\
\hline
\end{tabular}

${ }^{\mathrm{a}}$ Crepp et al. (2014).

b Tokovinin (2014).

${ }^{\mathrm{c}}$ Matthews et al. (2014).

$\mathrm{d}_{\text {van Leeuwen (2007). }}$

${ }^{\dagger}$ Triple or higher-order system.

HIP 83276: $R V$ curvature only. Although the HARPS radial velocities suggest the presence of a stellar mass companion, we do not have enough RV data points to infer any information about the orbital parameters of the system. Using radial velocities measured with the CORAVEL spectrograph, Duquennoy \& Mayor (1991) found the companion has $m \sin i=0.24 \mathrm{M}_{\odot}, e=0.185$ and an orbital period of 386.72 days.

HIP 87769: $R V$ curvature only. It is reported as a binary system by T14 but, similarly to HIP 54102, lacks an inflection point in its RV data from HARPS, which spans 3.3 yr. There is a wide range of possible orbital solutions that suggest $m \sin i$ varying from brown dwarf masses to $\sim 1 \mathrm{M}_{\odot}$. Higher eccentricities $(e>0.8)$ can be ruled out as unlikely because they suggest a companion with $m \sin i \approx 1 \mathrm{M}_{\odot}$ at an orbital period of more than $500 \mathrm{yr}$ and $a>80 \mathrm{AU}$.

\subsection{Considerations on multiplicity statistics}

Although planet search surveys are generally biased against the presence of binaries due to avoiding known compact multiple systems, the fraction of binary or higher-order systems in the whole sample of the Solar Twin Planet Search program is $42 \% \pm 6 \%^{8}$. This value agrees with previous mul-

8 Counting stellar and brown dwarf companions. The uncertainty is computed using a bootstrap resampling analysis with 10,000 iterations, similarly to Raghavan et al. (2010). In each iteration, a new set of 81 solar twins is randomly drawn from the original sample allowing stars to be selected more than once. 
tiplicity fractions reported by, e.g., Raghavan et al. (2010) and T14; however, it is signficantly lower than the 58\% multiplicity factor for solar-type stars reported by Fuhrmann et al. (2017), who argues that previous results are subject to selection effects and are thus biased against the presence of multiple systems.

The orbital period vs. mass ratio plot of companions in the Solar Twin Planet Search is shown in Fig. 7. A comparison with the sample of solar-type stars from T14 reveals two important biases in our sample: i) Mass ratios are mostly below 0.3 because of selection of targets that do not show large radial velocity variations in previous studies; ii) Orbital periods are mostly lower than $30 \mathrm{yr}$ because longer values cannot be constrained from the recent RV surveys targeting solartype stars with low-mass companions. In such cases, further monitoring of linear trend and RV curvature-only binaries may prove useful to understand the origins of the brown dwarf desert (Grether \& Lineweaver 2006). These targets are particularly appealing because the long periods mean that the separation from the main star is large enough to allow us to observe them directly using high-resolution imaging.

Previous studies on the period-eccentricity relation for binary stars found that systems with orbital periods below 10 days tend to have eccentricities near zero, while those between 10 and 1000 days follow a roughly flat distribution of eccentricities (Kjurkchieva et al. 2016, and references therein), an effect that is due to the timescales for circularization of orbits. In relation to our sample, with the exception of HIP 30037, HIP 65708 and HIP 83276, all of the binaries we observed have periods longer than 1000 days and eccentricities higher than 0.3 , which agrees with the aforementioned findings. According to Duquennoy \& Mayor (1991), the distribution of eccentricities on systems with $T>1000 \mathrm{~d}$ is a function of energy only, and does not depend on $T$ (see fig. 5 in Duquennoy \& Mayor). Interestingly, HIP 30037, which hosts a brown dwarf companion with $T=31.6 \mathrm{~d}$, falls inside the 25-35 days interval of orbital periods found by Kjurkchieva et al. that corresponds to a short stage of evolution of binaries undergoing a fast change in their orbits.

\section{CONCLUSIONS}

The Solar Twin Planet Search and several other programs observed 81 solar twins using the HARPS spectrograph. In total, 18 of these solar twins are spectroscopic binaries, 18 are visual binaries, and two intersect these categories. We found a multiplicity fraction of $42 \% \pm 6 \%$ in the whole sample, which is lower than the expected fraction ( $58 \%)$ because of selection effects that are generally seen in exoplanet search surveys.

We updated or reproduced the solutions of several known binaries, and determined all the orbital parameters of HIP 19911, HIP 65708, HIP 67620, HIP 79578, HIP 81746 and HIP 103983. The stars HIP 43297 and HIP 64673, which we previously reported as binaries, are likely to host longperiod giant planets instead of stellar companions. For binaries with partial orbits, we were able to place lower limits for some of their orbital parameters owing to the presence of curvature or an inflection point in their RV data. We estimated the most likely mass of the companions of the binaries that display only linear trends in their RV data. Future work

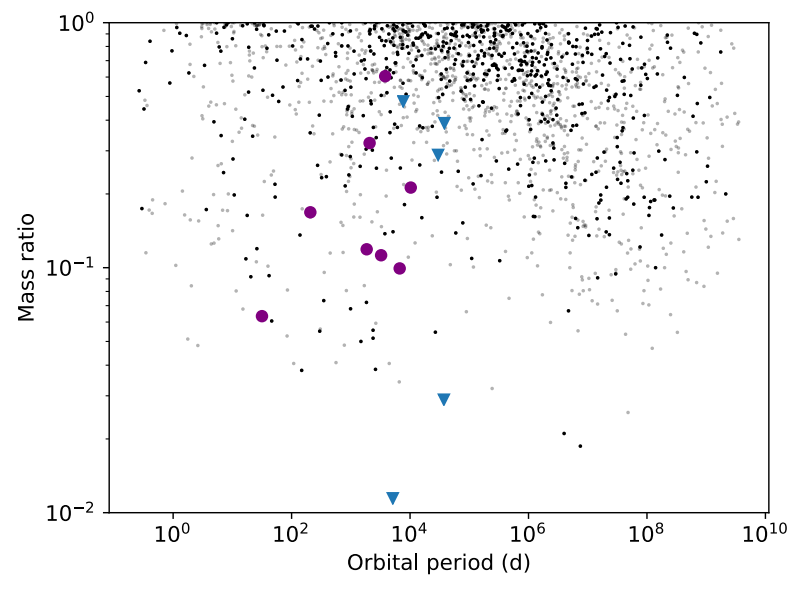

Figure 7. Mass ratios in function of the orbital periods of binary stars or higher-order systems in the solar neighborhood. The purple circles are binaries in our sample with well defined period and $m \sin i$; the blue triangles correspond to the binaries in our sample for which we only have lower limits for the periods and $m \sin i$. The stars from T14 are plotted as black dots (the darker ones are those with main star masses between 0.9 and $1.1 \mathrm{M}_{\odot}$ ).

is needed on studying the long-period binaries using photometry data and high-resolution imaging in order to constrain the nature of their companions. These wide solar twin binaries are prime targets for detailed physical characterization of their companions owing to the favorable separation for $\mathrm{AO}$ imaging and the precision with which we can measure the stellar parameters of the main star - this is particularly important for fully convective red dwarf stars and very lowmass companions such as the T dwarf HIP 14501 B, whose evolution and structure is still poorly constrained.

Additionally, we reported the detailed discovery of new companions to the following solar twins: HIP 6407, HIP 30037, HIP 54582, and HIP 62039, for which we are able to determine an orbital solution for the first two using radial velocities. The latter two do not have enough RV data to obtain precise orbital parameters, but we can nonetheless estimate their minimum companions masses. We found that these new companions are likely very low-mass, ranging from 0.02 to $0.12 \mathrm{M}_{\odot}$ (although stressing that these are lower limits), which should be useful in understanding the origins of the brown dwarf desert in future research.

The anomalies and RV residuals observed on HIP 19911, HIP 67620 and HIP 103983 are likely due to contamination by the companion on the spectra of the main star. Although the peculiar stars in our sample are no longer considered blue straggler candidates, it is important to note that the detection of WD companions is particularly important for the study of field Sun-like stars because they allow the estimation of their cooling ages; these are more reliable than isochronal and chromospheric ages in some cases, providing thus robust tests for other age estimate methods. We do not expect that the presence of $M$ dwarf companions contaminate lithium spectral lines in Sun-like stars, thus stellar ages derived from $\mathrm{Li}$ abundances may be more reliable for double-lined solar twins. We recommend a revision of the stellar parameters of the peculiar binary stars by analyzing high-resolution spectra at the highest Doppler separations 
possible, or using Gaussian processes to disentangle the contaminated spectra (see, e.g., Czekala et al. 2017).

We conclude that single-lined solar twin binaries with orbital periods larger than several months and moderate to low eccentricities do not display signals of distinct rotational evolution when compared to single solar twins. The most compact system in our sample, HIP 30037, which hosts a $0.06 \mathrm{M}_{\odot}$ brown dwarf companion at an orbital period of 31 days is, in fact, one of the quietest stars in the sample (in regards of its activity levels), and is thus a viable target for further efforts in detecting moderate- to long-period circumbinary planets.

\section{ACKNOWLEDGEMENTS}

LdS acknowledges the financial support from FAPESP grants no. 2016/01684-9 and 2014/26908-1. JM thanks FAPESP (2012/24392-2) for support. LS acknowledges support by FAPESP (2014/15706-9). This research made use of SciPy (Jones et al. 2001), Astropy (Astropy Collaboration et al. 2013), Matplotlib (Hunter 2007), and the SIMBAD and VizieR databases (Wenger et al. 2000; Ochsenbein et al. 2000), operated at CDS, Strasbourg, France. We thank R. P. Butler, S. Vogt, G. Laughlin and J. Burt for allowing us to analyze the LCES HIRES/Keck data prior to publication. LdS also thanks B. Montet, J. Stürmer and A. Seifahrt for the fruitful discussions on the results and code implementation. We would also like to thank the anonymous referee for providing valuable suggestions to improve this manuscript.

\section{REFERENCES}

Abt H. A., Willmarth D., 2006, ApJS, 162, 207

Astropy Collaboration et al., 2013, A\&A, 558, A33

Baranne A., et al., 1996, A\&AS, 119, 373

Barnes S. A., 2003, ApJ, 586, 464

Baron F., et al., 2015, ApJ, 802, 37

Baumann P., Ramírez I., Meléndez J., Asplund M., Lind K., 2010, A\&A, 519, A87

Bedell M., Meléndez J., Bean J. L., Ramírez I., Leite P., Asplund M., 2014, ApJ, 795, 23

Bedell M., et al., 2015, A\&A, 581, A34

Butler R. P., et al., 2017, AJ, 153, 208

Charbonneau P., 1992, in Giampapa M. S., Bookbinder J. A., eds, Astronomical Society of the Pacific Conference Series Vol. 26, Cool Stars, Stellar Systems, and the Sun. p. 416

Crepp J. R., Johnson J. A., Howard A. W., Marcy G. W., Gianninas A., Kilic M., Wright J. T., 2013, ApJ, 774, 1

Crepp J. R., Johnson J. A., Howard A. W., Marcy G. W., Brewer J., Fischer D. A., Wright J. T., Isaacson H., 2014, ApJ, 781 , 29

Crepp J. R., et al., 2015, ApJ, 798, L43

Cuntz M., Saar S. H., Musielak Z. E., 2000, ApJ, 533, L151

Czekala I., Mandel K. S., Andrews S. M., Dittmann J. A., Ghosh S. K., Montet B. T., Newton E. R., 2017, ApJ, 840, 49

Desidera S., D'Orazi V., Lugaro M., 2016, A\&A, 587, A46

Duquennoy A., Mayor M., 1991, A\&A, 248, 485

Eastman J., Gaudi B. S., Agol E., 2013, PASP, 125, 83

Feng Y. K., Wright J. T., Nelson B., Wang S. X., Ford E. B., Marcy G. W., Isaacson H., Howard A. W., 2015, ApJ, 800, 22

Figueira P., et al., 2016, A\&A, 592, A143

Fischer D. A., Marcy G. W., Spronck J. F. P., 2014, ApJS, 210, 5
Foreman-Mackey D., Hogg D. W., Lang D., Goodman J., 2013, PASP, 125, 306

Freitas F. C., et al., 2017, The Solar Twin Planet Search. VI. The age-chromospheric activity relation, submitted

Fuhrmann K., Chini R., Kaderhandt L., Chen Z., 2017, ApJ, 836, 139

Gallet F., Bouvier J., 2013, A\&A, 556, A36

Gray D. F., 2005, The Observation and Analysis of Stellar Photospheres

Grether D., Lineweaver C. H., 2006, ApJ, 640, 1051

Hartkopf W. I., Tokovinin A., Mason B. D., 2012, AJ, 143, 42

Haywood R. D., et al., 2016, MNRAS, 457, 3637

Hunter J. D., 2007, Computing In Science \& Engineering, 9, 90

Jenkins J. S., et al., 2015, MNRAS, 453, 1439

Jones E., Oliphant T., Peterson P., et al., 2001, SciPy: Open source scientific tools for Python, http://www.scipy.org/

Karoff C., Albrecht S., Bonanno A., Faurschou Knudsen M., 2016, ApJ, 830, L7

Kawaler S. D., 1988, ApJ, 333, 236

Kiseleva-Eggleton L., Eggleton P. P., 2001, in Podsiadlowski P., Rappaport S., King A. R., D'Antona F., Burderi L., eds, Astronomical Society of the Pacific Conference Series Vol. 229, Evolution of Binary and Multiple Star Systems. p. 91

Kjurkchieva D., Vasileva D., Dimitrov D., 2016, AJ, 152, 189

Latham D. W., Stefanik R. P., Torres G., Davis R. J., Mazeh T., Carney B. W., Laird J. B., Morse J. A., 2002, AJ, 124, 1144

Lovis C., et al., 2011, preprint, (arXiv:1107.5325)

Luhman K. L., 2014, ApJ, 781, 4

Makarov V. V., Kaplan G. H., 2005, AJ, 129, 2420

Matthews C. T., et al., 2014, ApJ, 783, L25

Meléndez J., et al., 2017, A\&A, 597, A34

Mengel M. W., et al., 2017, MNRAS, 465, 2734

Mestel L., 1984, Philosophical Transactions of the Royal Society of London Series A, 313, 19

Moultaka J., Ilovaisky S. A., Prugniel P., Soubiran C., 2004, PASP, 116, 693

Murray C. D., Correia A. C. M., 2010, Keplerian Orbits and Dynamics of Exoplanets. pp 15-23

Newville M., Stensitzki T., Allen D. B., Rawlik M., Ingargiola A., Nelson A., 2016, Lmfit: Non-Linear Least-Square Minimization and Curve-Fitting for Python, Astrophysics Source Code Library (ascl:1606.014)

Ochsenbein F., Bauer P., Marcout J., 2000, A\&AS, 143, 23

Paxton B., Bildsten L., Dotter A., Herwig F., Lesaffre P., Timmes F., 2011, ApJS, 192, 3

Paxton B., et al., 2015, ApJS, 220, 15

Perruchot S., et al., 2011, in Techniques and Instrumentation for Detection of Exoplanets V. p. 815115 (arXiv:1110.2256), doi: $10.1117 / 12.892466$

Queloz D., et al., 2001, A\&A, 379, 279

Raghavan D., et al., 2010, ApJS, 190, 1

Ramírez I., Allende Prieto C., Lambert D. L., 2013, ApJ, 764, 78

Ramírez I., et al., 2014, A\&A, 572, A48

Riddle R. L., et al., 2015, ApJ, 799, 4

Takeda Y., Tajitsu A., Honda S., Kawanomoto S., Ando H., Sakurai T., 2011, PASJ, 63, 697

Tokovinin A., 2014, AJ, 147, 86

Torres G., 1999, PASP, 111, 169

Tucci Maia M., Ramírez I., Meléndez J., Bedell M., Bean J. L., Asplund M., 2016, A\&A, 590, A32

Wenger M., et al., 2000, A\&AS, 143, 9

Willmarth D. W., Fekel F. C., Abt H. A., Pourbaix D., 2016, AJ, 152, 46

Wilson P. A., et al., 2016, A\&A, 588, A144

Yana Galarza J., Meléndez J., Ramírez I., Yong D., Karakas A. I., Asplund M., Liu F., 2016, A\&A, 589, A17

Yi S., Demarque P., Kim Y.-C., Lee Y.-W., Ree C. H., Lejeune T., Barnes S., 2001, ApJS, 136, 417 
dos Santos L. A., et al., 2016, A\&A, 592, A156

van Leeuwen F., 2007, A\&A, 474, 653

This paper has been typeset from a $\mathrm{T}_{\mathrm{E}} \mathrm{X} / \mathrm{LAT}_{\mathrm{E}} \mathrm{X}$ file prepared by the author. 